\title{
Balance of Plant System Analysis and Component Design of Turbo-Machinery for High Temperature Gas Reactor Systems
}

\author{
Final Report
}

May 14, 2003

NERI Project Number DE-FG07-00SF22171

\author{
Principal Investigators: $\quad$ Ronald G. Ballinger, Andrew Kadak, Neil Todreas \\ Massachusetts Institute of Technology \\ 77 Massachusetts Avenue \\ Cambridge, MA 02139 \\ Tel: 617-253-5118 \\ Fax: 617-253-0807 \\ Email: hvymet@mit.edu, kadak@mit.edu, todreas@mit.edu \\ Graduate Student \\ Mr. Chun Yun Wang \\ Collaborating Organization: Concepts: Northern Engineering \& Research \\ 39 Olympia Drive \\ Woburn, MA 01810 \\ Tel: 781-935-9050 \\ Fax: 718-935-9052 \\ Bradley Mirick, Eli Demetri, Martin Koronowski
}


Table of Contents

$\begin{array}{ll}\text { Executive Summary } & 4\end{array}$

1.0 Introduction and R\&D Plan 6

References 9

2.0 Background: Htgr Gas Turbine Power Plant 10

2.1 Introduction 22

2.2 High Temperature Gas Cooled Reactor $\quad 10$

2.2.1 Reactor System 10

2.2.2 Reactor Control 12

2.3 Power Conversion System 13

2.3.1 Gas Turbine Power Conversion System 13

2.3.2 Helium Gas Turbo-machines 14

2.3.3 Compact Heat Exchanger 17

2.3.4 Control Methods For Gas Turbine Power Conversion System 19

2.4 Advanced Gas Cooled Reactor Design Requirements 21

2.5 Overall Development Path 23

$\begin{array}{ll}\text { References } & 24\end{array}$

3.0 Results: Gas Turbine Power Conversion System Design Considerations 26

$\begin{array}{ll}3.1 \text { Introduction } & 26\end{array}$

3.2 General Design Consideration $\quad 26$

3.3 Design Constraints 30

3.4 Configuration Consideration $\quad 31$

3.5 Compact Heat Exchanger And Helium Gas Turbomachine Design Considerations. 33

3.6 Schematic Of Current Design $\quad 41$

3.7 Summary $\quad 42$

$\begin{array}{ll}\text { References } & 44\end{array}$

4.0 Results: Model Development 45

4.1 Introduction $\quad 45$

4.2 Steady State Model Development $\quad 45$

4.2.1 Component Losses 45

4.2.2 Steady State Cycle Calculation Procedure 52

4.2.3 Estimation Of Cooling Mass Flowrate For RPV And IHX Vessel 55

4.3 Dynamic Model Development $\quad 60$

$\begin{array}{ll}\text { 4.3.1 Solution Approach } & 60\end{array}$

4.3.2 Sub-Models Of Components $\quad 62$

4.3.2.1 Reactor Model $\quad 62$

4.3.2.2 Heat Exchanger Model 78

4.3.2.3 Turbo-machinery Model $\quad 82$

4.3.2.4 Shaft And Generator Model 89

4.3.2.5 Valve Model 90 
4.3.2.6 Pipe Model $\quad 90$

4.3.2.7 Pi Controller 91

4.3.3 Integration Of Component Sub-Models 91

4.4 Summary

92

References

5.0 Results: Control System Design $\quad 96$

5.1 Introduction $\quad 96$

5.2 Control Strategy $\quad 96$

5.3 Control Methods 98

$\begin{array}{ll}5.4 \text { Configuration Of Control System } & 100\end{array}$

5.5 Automatic Control System 101

5.6 Summary 102

References 103

$\begin{array}{ll}\text { 6.0 Results: Plant Analysis } & 104\end{array}$

6.1 Introduction 105

6.2 Steady State Parametric Analysis 106

6.3 Transient Analysis $\quad 113$

6.3.1 Verification With Flownet Model 113

6.3.2 10\% Load Step Change - Bypass Valve Control Used 115

$\begin{array}{ll}\text { 6.3.3 Grid Separation } & 139\end{array}$

6.4 Conclusion 141

6.4.1 Cycle Design Results 141

6.4.2 Control System Results 143

6.5 Summary 143

References 146

$\begin{array}{ll}\text { 7.0 Results: Summary And Discussion } & 147\end{array}$

$\begin{array}{ll}\text { 7.1 Summary Of Conclusions } & 147\end{array}$

7.2 Discussion And Recommendations 148

$\begin{array}{ll}\text { Appendix A Concepts-Nrec Heat Exchanger Design } & 150\end{array}$

$\begin{array}{ll}\text { Appendix B Concepts-Nrec Turbomachinery Design } & 161\end{array}$

Appendix C Intermediate Heat Exchanger Assembly Design 178

Appendix D Thermodynamic And Transport Properties Of Helium 182

$\begin{array}{lr}\text { Appendix E Nomenclature } & 183\end{array}$ 


\section{Executive Summary}

The Modular Pebble Bed Reactor system (MPBR) requires a gas turbine cycle (Brayton cycle) as the power conversion system for it to achieve economic competitiveness as a Geneneration IV nuclear system. The availability of controllable helium turbomachinery and compact heat exchangers are thus the critical enabling technology for the gas turbine cycle. The development of an initial reference design for an indirect helium cycle has been accomplished with the overriding constraint that this design could be built with existing technology and complies with all current codes and standards. Using the initial reference design, limiting features were identified. Finally, an optimized reference design was developed by identifying key advances in the technology that could reasonably be expected to be achieved with limited R\&D. This final reference design is an indirect, intercooled and recuperated cycle consisting of a three-shaft arrangement for the turbomachinery system.

A critical part of the design process involved the interaction between individual component design and overall plant performance. The helium cycle overall efficiency is significantly influenced by performance of individual components. Changes in the design of one component, a turbine for example, often required changes in other components. To allow for the optimization of the overall design with these interdependencies, a detailed steady state and transient control model was developed. The use of the steady state and transient models as a part of an iterative design process represents a key contribution of this work.

A dynamic model, MPBRSim, has been developed. The model integrates the reactor core and the power conversion system simultaneously. Physical parameters such as the heat exchangers' weights and practical performance maps such as the turbine characteristics and compressor characteristics are incorporated into the model. The individual component models as well as the fully integrated model of the power conversion system have been verified with an industrystandard general thermal-fluid code Flownet.

With respect to the dynamic model, bypass valve control and inventory control have been used as the primary control methods for the power conversion system. By performing simulation using the dynamic model with the designed control scheme, the combination of bypass and inventory control was optimized to assure system stability within design temperature and pressure limits. Bypass control allows for rapid control system response while inventory control allows for ultimate steady state operation at part power very near the optimum operating point for the system. Load transients simulations show that the indirect, three-shaft arrangement gas turbine power conversion system is stable and controllable.

For the indirect cycle the intermediate heat exchanger (IHX) is the interface between the reactor and the turbomachinery systems. As a part of the design effort the IHX was identified as the key component in the system. Two technologies, printed circuit and compact plate-fin, were investigated that have the promise of meeting the design requirements for the system. The reference design incorporates the possibility of using either technology although the compact plate-fin design was chosen for subsequent analysis. The thermal design and parametric analysis 
with an IHX and recuperator using the plate-fin configuration have been performed. As a threeshaft arrangement, the turbo-shaft sets consist of a pair of turbine/compressor sets (high pressure and low pressure turbines with same-shaft compressor) and a power turbine coupled with a synchronous generator. The turbines and compressors are all axial type and the shaft configuration is horizontal. The core outlet/inlet temperatures are $900 / 520^{\circ} \mathrm{C}$, and the optimum pressure ratio in the power conversion cycle is 2.9. The design achieves a plant net efficiency of approximately $48 \%$. 


\subsection{Introduction}

The purpose of this project was to develop systems analysis tools for the evaluation of turbomachinery and BOP power conversion in high temperature gas cooled reactor systems. These tools were then used to develop optimized power conversion systems for high temperature gascooled reactor systems. Current concepts for high temperature gas cooled reactor systems call for modular designs with electrical output in the 110 MWe range. Key questions which needed to be addressed in order for such systems to be adequately evaluated included: (1) could a helium power turbine be developed in the $110 \mathrm{MWe}$ range, (2) could advanced compact heat exchanger technology be used in the design of intermediate heat exchangers (for indirect cycle plants) and/or recuperators (direct and indirect cycle plants), (3) could structural and materials issues be adequately characterized to allow for detailed life-cycle analysis, (4) how did specific component designs impact overall cost.

This report presents the results of this program. Key results include the following:

1. We have established a "reference" plant design which can be built with existing technology. However, the thermal efficiency of the plant will be sub-optimal due to restrictions on the reactor outlet temperature.

2. We have established initial designs for the turbo machinery and heat exchangers, including the intermediate heat exchanger. Two concepts have been identified. However, each design will require that active measures be taken to assure safe operation.

3. We have identified potential vendors for all of the major components and have obtained initial cost estimates for these components.

4. We have developed a steady state model for the overall plant and are in the process of using this model to help optimize the system configuration.

5. We have developed the initial transient model and are in the process of refining the individual component models.

6. We have developed a reactor model for use in both the steady state and transient models.

7. We have made use of the above results to develop an optimized system.

High temperature, gas cooled reactor systems have been identified as candidate "Generation IV" reactor systems. Gas cooled reactor systems promise increased efficiency, simplification of design (fewer components), easier (and less frequent) maintenance, improved reliability, improved safety and, as a result of these characteristics, reduced cost. So great is the promise for these systems that international development of prototype systems is being carried out in several countries including Japan, China, and South Africa. In South Africa, work is in progress on the design and construction of a "commercial" prototype which will serve as the first of a kind power production reactor. The South African effort has attracted outside investors including British Nuclear Fuels (BNFL).

In spite of the general optimism regarding gas reactor systems, the experience to date has been mixed with regard to past performance. In Germany experience with operation of the AVR was 
excellent until the plant was shut down after approximately 20 years of operation [1]. A larger plant was planned and built but experienced operational difficulties and was shut down. In the US a prototype and a commercial power reactor have been operated. The commercial reactor, Fort St. Vrain, was operated for several years before it was shut down due to difficulties with primary circulators and other components[1]. Other, smaller gas cooled reactors have been explored as power sources for space propulsion systems but have not been deployed. The well known MAGNOX reactor systems were, at one time, the largest source of electric power produced from nuclear sources although these systems were not classified as high temperature systems.

The higher efficiency of the gas reactor systems is achieved due to the use of helium as the primary coolant. The choice of helium allows for the use of superheated steam in the secondary plant if a steam cycle is desired, as was the case with Fort St. Vrain and the AVR. However, the use of a conventional secondary (steam) cycle requires that the safety analysis address the issue of water ingress to the primary system during accident conditions. Additionally, water ingress during plant operation is a real possibility and poses a serious threat to plant reliability. Lastly, the use of a conventional steam cycle for the secondary plant forces the operator to deal with the same environmental degradation issues that have plagued Light Water Reactor (LWR) systems.

An alternative to the use of a steam cycle for the secondary plant is to use gas as the working fluid. The use of gas allows (actually requires) the use of a Brayton cycle for the power conversion process. In it's simplest incarnation, the Brayton cycle technology for a gas reactor is not significantly different from that of conventional gas turbine technology. Thus, it's proponents claim, use can be made of the extremely large body of experience which has been gained in the gas turbine industry. However, this claim needs to be justified. Indeed, the cycle is formally the same. However, the use of a nuclear heat source places constraints on the system design which do not exist with an open cycle gas turbine. Foremost of these constraints is the requirement that release of radioactivity be held to extremely low levels. This requirement effectively eliminates the open cycle from consideration since the current state of the art in fuel reliability is not sufficient to insure low release during normal operation, let alone during accident conditions. Additionally, the use of an open cycle results in extremely large components, especially the recuperator, which destroys the economic advantage of the system.

For the above reasons, a closed cycle is the most appropriate cycle when a nuclear heat source is used. Two basic cycles have been explored to a greater or lesser degree: direct and indirect. The direct cycle, as the name implies, circulates the hot gas from the nuclear core directly to the power conversion system. The indirect cycle places an intermediate heat exchanger between the nuclear heated gas and the gas used in the power conversion system. The direct cycle has the advantage of being simpler, having fewer components (sans the intermediate heat exchanger), and should have a higher efficiency. It's disadvantage is that contamination of the power conversion circuit would likely complicate maintenance. Additionally, from a design standpoint, the use of a direct cycle requires that the entire pressure boundary satisfy the ASME Section III, Class I pressure boundary criteria. In the area of safety, the direct cycle must consider air/water ingress as at least a hypothetical accident scenario as well as the possibility of foreign object damage to the core and structure in the event of power conversion cycle component failure. The indirect cycle isolates, in principal, the potentially contaminated primary system from the 
secondary power conversion system, making maintenance easier. The indirect cycle also offers the possibility of reducing the Class I pressure boundary area-allowing the "secondary" side to be built to ASME Section VIII criteria. Section 8 allows for higher temperature operation and admits a wider range of materials choices. Additionally, the air/water ingress accident is, for all practical purposes, not credible. Foreign object damage to the core and structural components is also effectively eliminated. However, the intermediate heat exchanger, which is the Section IIISection VIII boundary, can be very large and it's reliability will have to be very high. Previous, and there have been many, design studies related to gas reactor systems have been essentially silent on the subject of heat exchanger reliability.

A second significant roadblock in the road to the application of industrial gas turbine technology to the case of high temperature gas reactor systems relates to the turbo-machinery. While there exists a large body of industrial gas turbine experience, this experience is limited to two general classes of machines: (1) large, "frame" type machines with low operating pressure and low pressure ratio and, (2) small open or closed cycle, marine/aviation gas turbines which operate at high pressure and with a high pressure ratio. Unfortunately, for high temperature gas reactor applications, the most appropriate system is a larger, high pressure, closed cycle system, with a relatively low pressure ratio. This type of machine falls in between the two main bodies of experience. Thus, while there is some experience with helium turbines with a similar operating pressure and pressure ratio, the power rating of these turbines has not been at the level anticipated $(\sim 100 \mathrm{MW})$ for gas reactor systems. The current maximum shaft horsepower available as an "off the shelf item" is limited to approximately 50MW (70,000 shaft horsepower). The only large frame helium turbine was built in Germany (Obrighausen) at 50MW but sized for a larger capacity. This facility, while it operated successfully, was difficult to operate (high maintenance) and did not achieve its efficiency goals.

In this project we extend the previous work in the field beyond the conceptual design and analysis of turbo machinery by a significant step-to bring the design to the point where key questions related to the design of gas reactor turbo and thermal machinery can be answered.

Key questions which are being addressed in this project are: (1) can a helium power turbine be developed in the $110 \mathrm{MWe}$ range, (2) can advanced compact heat exchanger technology be used in the design of intermediate heat exchangers (for indirect cycle plants) and/or recuperators (direct and indirect cycle plants), (3) can structural and materials issues be adequately characterized to allow for detailed life-cycle analysis, (4) how do specific component designs that can actually be built effect overall cost.

\section{Research \& Development Plan}

The R\&D plan for the project is being carried out in six major tasks. These tasks are:

Task 1: Turbo-Machinery Design

Task 2: Heat Exchanger Design

Task 3: Structural and Materials Issues

Task 4: Life Cycle Analysis

Task 5: Economics 
The individual tasks are identified separately. However, due to the highly interactive nature of the design process, there will be significant overlap and dependency between tasks. As an example, structural and life cycle analysis will depend on individual component design and materials of construction which will then depend on temperature limitations for materials. Also, the optimization of the system design requires that a steady state and transient model be developed for the overall system. Thus, our focus has been two pronged. One prong focused on individual components. The second prong focused on the development of system models. Individual component models are then used in the system models. Due to the highly interactive nature of the system, this report is organized in a pedagogical fashion with respect to the system design. Results from all of the tasks are this folded into the overall report.

\section{References: Section 1}

1. McDonald, C.F., "The Nuclear Gas Turbine-Towards Realization After Half a Century of Evolution, International Gas turbine and Aeroengine Congress and Exposition, Houston, TX, June 5-8, 1995 


\section{Background: HTGR gas turbine power plant}

In this section, the world-wide development of the high temperature gas cooled reactor system is described briefly. Two core types, prismatic and pebble bed, are introduced. The control methods applying to the reactor and the power conversion system are discussed. After discussion of the gas turbine cycle, the technologies and experiences of heat exchanger and helium gas turbo-machinery are discussed. Finally, the requirement of the advanced gas cooled reactor system as a candidate "Generation IV" reactor system and the development path in this study are presented.

\subsection{The High Temperature Gas Cooled Reactor (HTGR)}

\subsubsection{Reactor System}

The first commercial gas-cooled power reactor began operation at Calder Hall in England in 1956 and produced $40 \mathrm{MW}$ of electricity. These first power reactors were graphite moderated with natural uranium metal fuel rods and cooled by circulating $0.8 \mathrm{MPa} \mathrm{CO}_{2}$ at an outlet temperature of $335^{\circ} \mathrm{C}$. These "Magnox" reactors, had a low power density -0.1 to 0.5 $\mathrm{MW}(\mathrm{e}) / \mathrm{m}^{3}$, using natural uranium as fuel and graphite as the moderator. To improve the thermodynamic efficiency and fuel utilization of the Magnox reactors, the Advanced Gas-Cooled Reactors (AGRs) were developed. In AGRs, 2.5\% $\mathrm{U}^{235}$ enriched $\mathrm{UO}_{2}$ pellets were used as fuel. This led to outlet temperature increases to $560^{\circ} \mathrm{C}$ from the 350 to $400{ }^{\circ} \mathrm{C}$ of the original Magnox reactors. 26 Magnox reactors and 14 AGRs have been constructed in the United Kingdom and 8 Magnox reactors in France[1,2]. With the experience of Magnox and AGR reactors, $\mathrm{CO}_{2}$ corrosion of the steel components and carbon corrosion by $\mathrm{CO}_{2}$ remain as areas of concern[1].

In parallel with the development of the AGR, in the mid-50s the idea for a high temperature gas cooled reactor (HTGR) was proposed. HTGRs utilize ceramic coated particle fuel, in which the $500 \mu \mathrm{m}$ diameter fuel particles are surrounded by coatings and dispersed in a graphite matrix. Helium is used as the coolant and graphite as the moderator. The combination of graphite core structure, ceramic fuel and inert helium permits very high operating temperature.

There are two core types for the HTGR - prismatic and pebble bed. For the prismatic core, the coated particles are loaded in cylindrical fuel compacts that are inserted in hexagonal graphite fuel elements, as shown in Figure 2.1[3]. The elements contain other holes for control rod insertion, flow of gas coolant and holding the burnable poison rods. The fuel elements are packed in the core and replaced as a batch when they are depleted. The prismatic type HTGRs have been constructed in the United Kingdom, the United States and more recently in Japan. The Dragon reactor in the United Kingdom was the first HTGR prototype, which operated first in July 1965 and was decommissioned in March 1976 after long full power operation [4]. The Peach Bottom Unit 1 was the first HTGR prototype in the United States. It achieved initial criticality in March 1966 and full power in May 1967. The plant went into commercial operation in June 1967 and was shut down for decommissioning in October 1974 [4]. This was followed by a large commercial prismatic plant, Fort St. Vrain in the United States. Fort St. Vrain was a steam cycle plant with a capacity of $842 \mathrm{MW}(\mathrm{t})$ and $330 \mathrm{MW}(\mathrm{e})$. Initial electric power generation was achieved at Fort St. Vrain in December 1976. It was shut down permanently in 
1990 due to its low availability primarily caused by problems with the water-lubricated bearing of the helium circulator [2,5]. Recently, the Japanese test reactor HTTR reached first criticality in 1998 and reached full power in 2001. It is a $30 \mathrm{MW}(\mathrm{t})$ prismatic core HTGR design with outlet temperature $850^{\circ} \mathrm{C}[6]$.

For the pebble bed core, the coated particles are embedded in spherical graphite fuel "pebbles" with a diameter of approximately $60 \mathrm{~mm}$, as shown in Figure 2.2[7]. One typical pebble contains ten to twenty thousand coated particles. The pebbles randomly packed in the core cavity forms the fuel system. Fresh pebbles are added to the top of the core and the burned pebbles are extracted at the bottom. After measuring the burnup, the partially burned pebbles are recycled to the top of the core for another cycle. The coolant flows through the interstices presented in the bed. The control rods have been inserted either directly into the core, or into the side reflector, depending the core size. Pebble bed reactors have been developed in Germany: the AVR test reactor and the THTR power plant. The AVR reached criticality in August 1966 and operated until December 1988 [8]. The THTR nuclear power plant included a steam cycle to generate a net output of $296 \mathrm{MW}$ electricity. The construction of THTR-300 began in 1971 and was completed in 1984. The plant was connected to the electrical grid of the utility in November 1985. It was shutdown permanently in 1989[8]. A pebble bed test reactor HTR-10 has been built in China $[9,10]$. It achieved its first criticality in December 2000 and was connected to the electrical grid of the utility in January 2003.

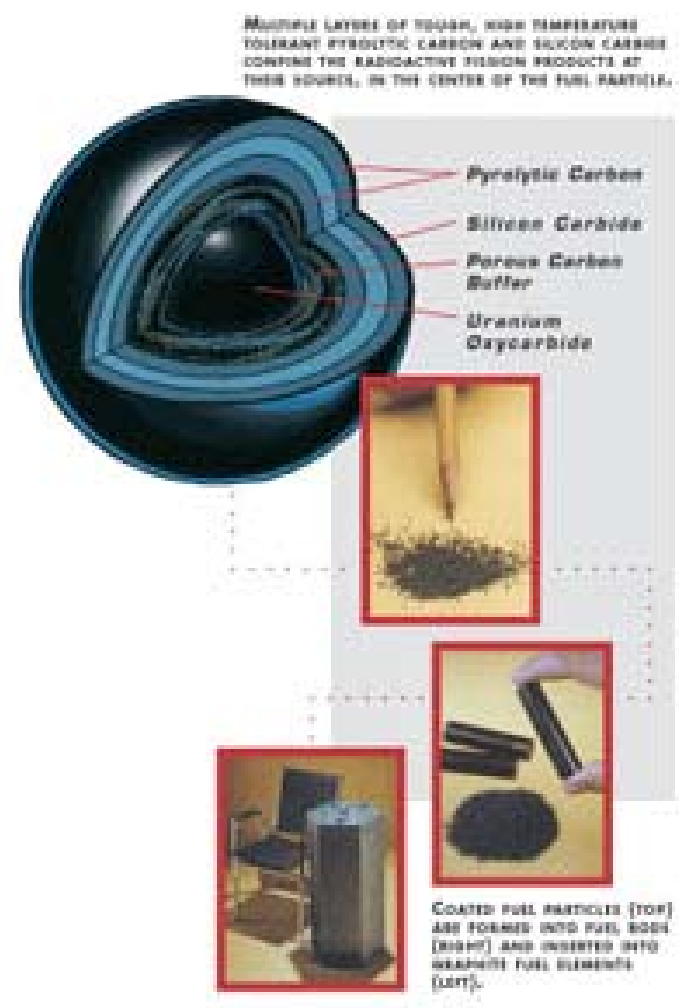

Figure 2.1 Hexagonal fuel element for prismatic core [3] 


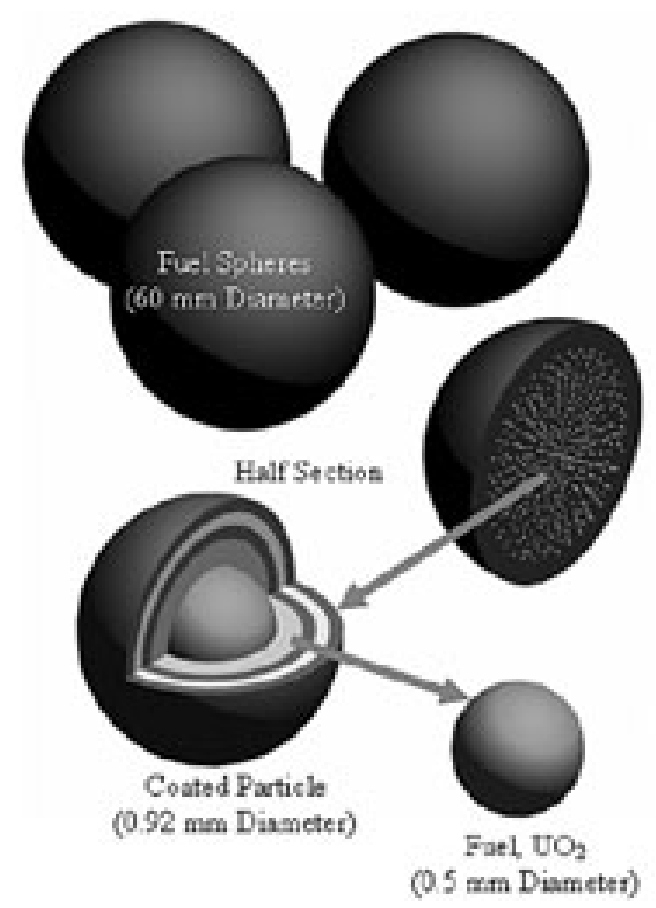

Figure 2.2 Pebble fuel element[7]

Excellent safety characteristics can be achieved for HTGRs due to their unique features: high heat capacity of the graphite core, chemical stability and inertness of the coolant, high retention capability of fission products in the fuel particles and the inherent negative temperature coefficient of reactivity of the core. With a deliberate decrease in the power level and reconfiguring of the reactor, the modular type HTGR concept was proposed in the early 1980s. The modular type HTGR provides the extra unique characteristic that the fuel temperature will not exceed the failure temperature following postulated accidents just by using passive heat transfer mechanisms. Currently, a joint United States - Russian Federation program is developing the GT-MHR project for burning weapon-grade plutonium [11, 12]. GT-MHR is a modular, prismatic reactor design with $600 \mathrm{MWt} / 286 \mathrm{MWe}$. Another commercial modular HTGR is being developing by ESKOM in South Africa - PBMR [7,13]. PBMR is a modular pebble bed reactor design with $265 \mathrm{MWt} / 116.3 \mathrm{MWe}$. Both GT-MHR and PBMR include a direct closed gas turbine cycle, which will be described in detail in the section 2.3. In this study, MPBR will use a pebble bed reactor core with thermal power of $250 \mathrm{MW}$ similar to the PBMR core.

\subsubsection{Reactor Control}

In the pebble bed reactor core, there are usually two reactivity control systems - the control rod system and the small absorber ball system, as shown in Figure $2.3[14,15,16]$. The control rod system consists of several control rods and the same number of drive mechanisms. It is usually utilized as the power regulating and control system and the first shutdown system as well. The control rod drive mechanism inserts the control rod into the side reflector and removes it out. For a large pebble bed reactor, the control rods can, in principle, be inserted into the reactor core. However, the disadvantages are that the rod insertion will interfere with the pebble fuel elements and may damage them. The small absorber ball system is the second shutdown system. If emergency shutdown is required and the control rod system cannot be assured to work, 
boronated (boron carbide) balls are dropped by gravity into side channels to shut down the system. In order to restart the system, the small absorber ball system provides means to remove the absorber balls from the channels and to put them back into the ball storage vessels.

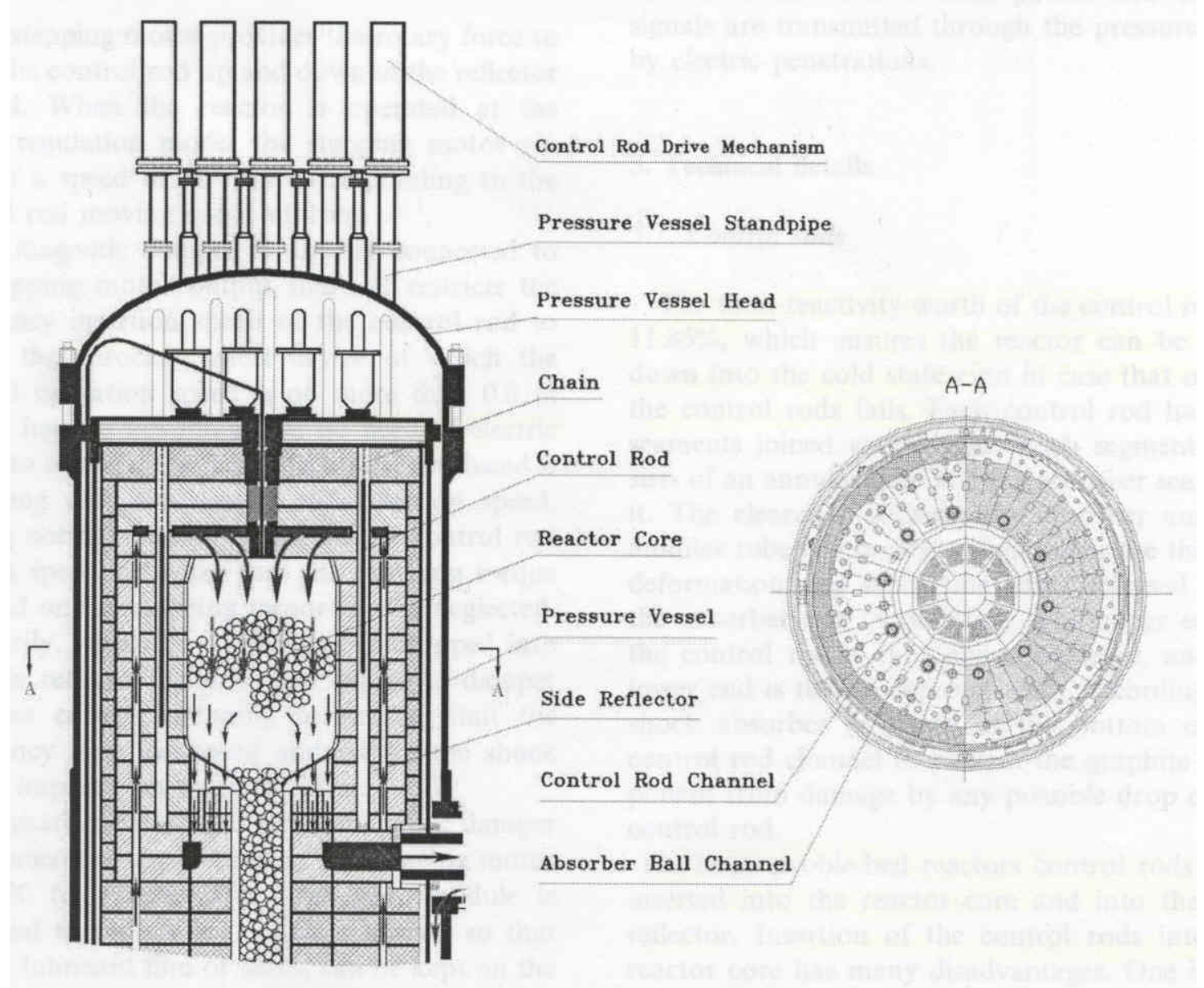

Figure 2.3 Control rod arrangement in the HTR-10 [14]

In the indirect cycle design, the circulator provides the pressure head for the helium to overcome the pressure losses through primary system. The mass flowrate in the primary system is proportional to the circulator speed. If the circulator speed is adjusted, the mass flowrate is changed correspondingly. Therefore, the mass flowrate in the primary system can be manipulated by adjusting the circulator speed. In the AVR and THTR, mass flow control in the primary system was achieved by varying the circulator speed [1].

\subsection{Power Conversion System}

\subsubsection{Gas Turbine Power Conversion System}


The advantages of coupling an HTGR with a closed Brayton cycle as the power conversion unit (PCU) have been recognized for many years. However, the actual system can not be realized until key technologies have been developed. The key technologies include:

(a) The size of gas turbines must be increased to accommodate the heat energy transformation proposed for a HTGR module;

(b) The technology for an effective compact heat exchanger must be available. The volume and capital cost must be reasonable;

(c) The feasibility of a large magnetic bearing must be demonstrated with current technology. Using magnetic bearings instead of oil-lubricated bearings obviates the oil-ingress problem which contaminates the helium coolant.

The gas turbine HTGR plant holds the promise for electricity generation with high efficiency. Reported expected efficiencies of $43 \%$ to $48 \%$ have been claimed $[12,13]$. Rankine cycle nuclear plants, such as the PWR and BWR, usually provide an efficiency of around $33 \%$ for electricity generation.

Both the conceptual designs of the GT-MHR and PBMR adopt a direct closed gas turbine cycle for the power conversion system. The PCU of the GT-MHR utilizes a single-shaft arrangement consisting of a turbine, an electric generator, and two gas compressors on a common, vertically oriented shaft supported by magnetic bearings. The PCU also includes a recuperator, precooler and intercooler[3,11]. In the PCU of the PBMR, there are three vertically oriented shafts. The high-pressure turbine drives the high-pressure compressor while the low-pressure turbine drives the low-pressure compressor. The power turbine drives the electric generator. Also, a recuperator, precooler and intercooler are used in the PBMR [7,13].

However, implementing the gas turbine nuclear plant depends on the technical feasibility of helium gas turbomachines and the compact heat exchanger. The following describes the technologies for helium gas turbomachines and compact heat exchanger.

\subsubsection{Helium Gas Turbomachines}

Gas turbines have been used throughout the world for marine/aviation propulsion and power generation in land based power plants for many years. Large scale gas turbine output power can be over 200MW for a land based power plant [17]. However, its working fluid is combustion gases (from the mixture of air and fuel, such as natural gas or oil). Experience with design and operation of closed cycle helium turbo-machinery has been finite but limited. Two large-scale helium facilities for testing closed cycle helium turbo-machinery have been operated in Germany: (1) the 50MW(e) Oberhausen 2 helium turbine plant (EVO), and (2) the high temperature helium test plant (HHV).

1. $50 \mathrm{MW}(\mathrm{e})$ Oberhausen 2 helium turbine plant (EVO)[18]

The design of the EVO test plant was for an electrical power of $50 \mathrm{MW}$ and heating (district heat) power $53.5 \mathrm{MW}$. The thermal heat source for the closed helium cycle was a fossil-fired heater. The basic flow scheme and design parameters are shown in Figure 2.4. A two-shaft 
arrangement was selected for the turbomachinery. The high-pressure (HP) turbine drives the low-pressure (LP) compressor and HP compressor on the first shaft with a rotational speed of 5,500rpm and the LP turbine drives the generator synchronizing with the grid (rotational speed 3,000rpm) on a separated shaft. Both shafts are interconnected by a gear. Since the power generated from the HP turbine is consumed by the compressors, there is not much power to transfer from the HP turbine to the generator through the gear. As shown in Figure 2.4, the HP turbine inlet temperature and pressure were $750{ }^{\circ} \mathrm{C}$ and $2.7 \mathrm{MPa}$, respectively. Helium mass flow rate for the cycle was $84.8 \mathrm{~kg} / \mathrm{s}$.

The power regulation of the EVO test plant uses the same principle adopted for closed cycle air turbine plants. Both inventory control and bypass valve control, which will be described in the next section, are used.

The HP turbine has 7 stages with 50\% reaction. The turbine rotor disc and the blade feet are cooled by extracting a helium stream from the HP compressor outlet. The LP turbine has 11 stages. One picture of the turbine is shown in Figure 2.5. The HP compressor and LP compressor have 15 stages and 10 stages, respectively, both with $100 \%$ reaction. Oil lubricatedlabyrinth seals are used for sealing. The housing and nozzles are also cooled.

The plant was connected to the grid on November, 1975. Up to the end of 1988, the helium turbine plant had been operated approximately 24,000 hours. A total of 11,500 hours operation had been at the design temperature of $750^{\circ} \mathrm{C}$. During operation many components and systems showed good performance. As the "first-of-a-kind" of a large helium turbine plant, some problems, such as vibration and low power output, arose unexpectedly for some components. The maximum electricity power output was $30.5 \mathrm{MW}$, which is much less than the design nominal data of 50MWe.

The HHV facility was built at KFA, Juelich, Germany for testing of large scale helium turbomachinery. The flow schematic and other circuit parameters are shown in Figure 2.6. Helium gas with a flow rate of approximately $200 \mathrm{~kg} / \mathrm{s}$ is circulated the system by means of electrically-driven turbomachinery. The compressor power is $90 \mathrm{MW}$, of which one part is provided by the turbine generation power of about $46 \mathrm{MW}$ and the difference is supplied by a 45 MW electric motor. As result of the compressor work, the helium is heated up to $850{ }^{\circ} \mathrm{C}\left(1000{ }^{\circ} \mathrm{C}\right.$ for short time periods) so that a fossil-fired heater is not needed. The system pressure is $5.0 \mathrm{MPa}$.

A two-stage turbine and an eight-stage compressor are on a shaft with a synchronous rotational speed of 3000rpm. The blade feet, rotor and housing are cooled by means of a cooling gas system or a sealing gas system. For the cooling gas system, radial-type compressors circulate the cooling helium of $56.8 \mathrm{~kg} / \mathrm{s}$ at an inlet temperature of $236^{\circ} \mathrm{C}$ and inlet pressure of $4.9 \mathrm{MPa}$ and an outlet pressure of $5.35 \mathrm{MPa}$ at $258^{\circ} \mathrm{C}$. The radial-type compressors are driven by a $6.5 \mathrm{MW}$ electric motor. 


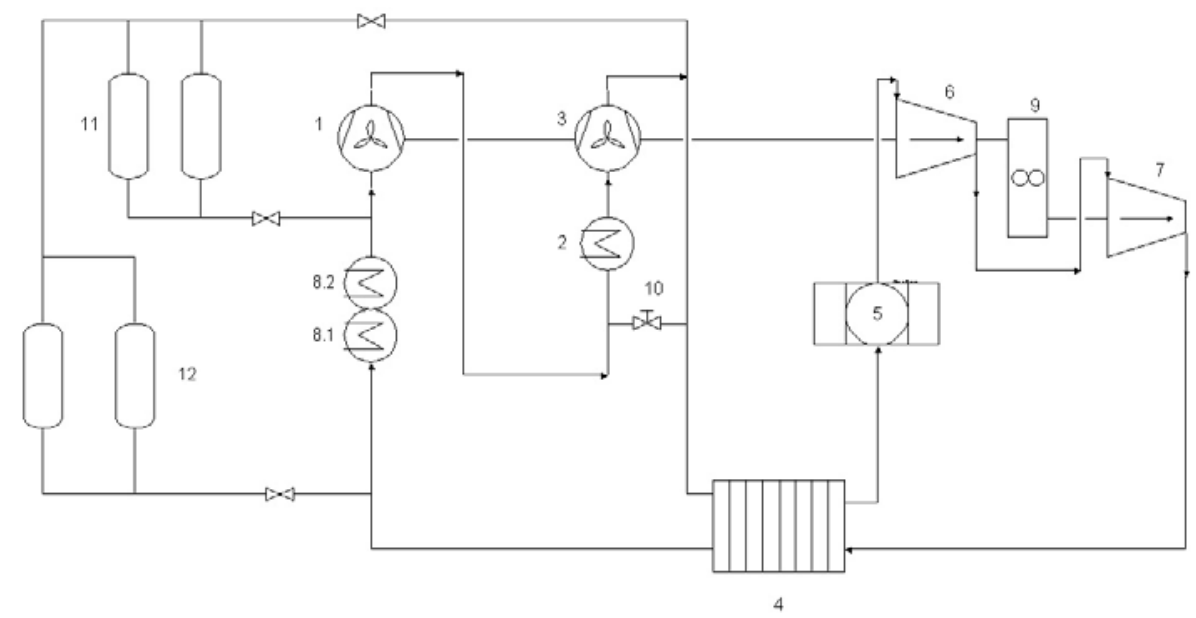

\begin{tabular}{|l|c|c|}
\hline & Inlet temperature $\left({ }^{\circ} \mathrm{C}\right)$ & Inlet pressure $(\mathrm{MPa})$ \\
\hline 1. LP compressor & 25 & 1.05 \\
\hline 2. Intercooler & 83 & 1.55 \\
\hline 3. HP compressor & 25 & 1.54 \\
\hline 4. Recuperator, HP side & 125 & 2.87 \\
\hline 5. Heater & 417 & 2.82 \\
\hline 6. HP turbine & 750 & 2.7 \\
\hline 7. LP turbine & 580 & 1.65 \\
\hline 8.1 Precooler (heating part) & 460 & 1.08 \\
\hline 8.2. Precooler (cooling part) & 169 & 1.06 \\
\hline 9. Gear & & \\
\cline { 1 - 3 } 10. Regulation bypass valve & & \\
\cline { 1 - 1 } 11. Storage reservoirs & & \\
\cline { 1 - 1 } 12. Transfer reservoirs & & \\
\hline
\end{tabular}

Figure 2.4 Oberhausen plant circuit, control and cycle parameters [18]

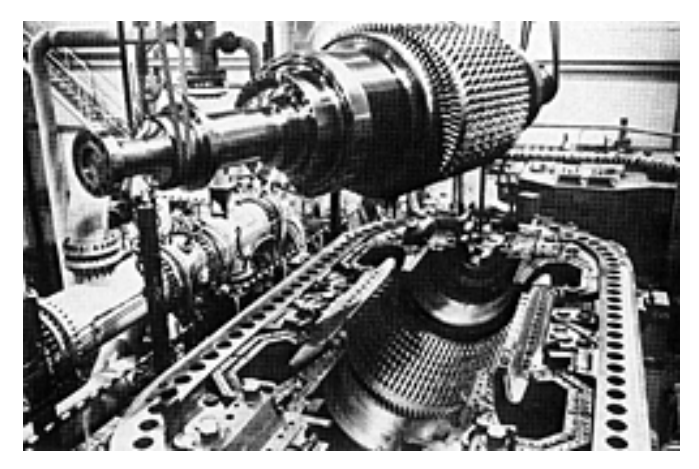

Figure 2.5 Oberhausen 2 helium turbine 
2. High temperature helium test facility (HHV) [18]

During the initial operation, oil ingress and excessive helium leakage occurred. After having overcome the initial problems, the HHV facility was successfully operated for about 1100 hours, of which it operated for about 325 hours at $850^{\circ} \mathrm{C}$. The measured results show that the compressor and turbine have a higher efficiency than the design value.

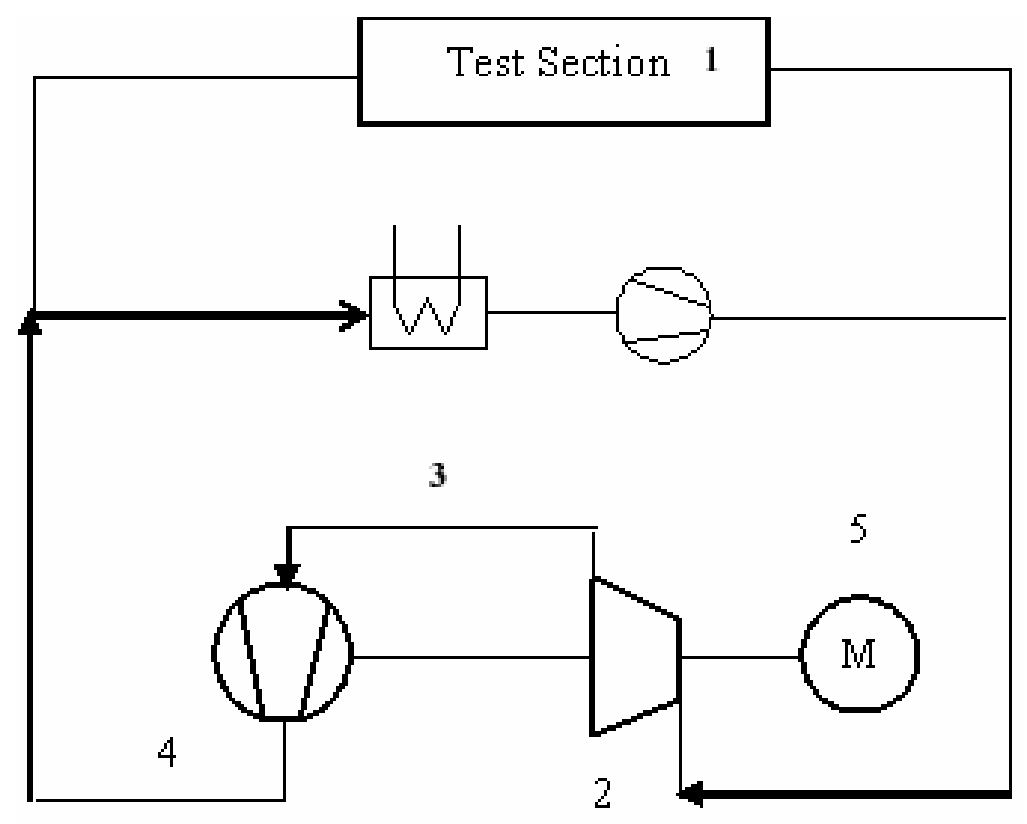

Legend: 1. Test section; 2. Helium turbine, 3. Duct to compressor inlet, 4. Compressor, 5. Electric motor

\begin{tabular}{|l|c|c|c|}
\hline & Temperature $\left({ }^{\circ} \mathrm{C}\right)$ & Pressure $(\mathrm{MPa})$ & Flow rate $(\mathrm{kg} / \mathrm{s})$ \\
\hline Compressor outlet & 850 & 5.12 & 212.0 \\
\hline Test section inlet & 850 & 5.12 & 201.0 \\
\hline Turbine inlet & 826 & 4.97 & 209.0 \\
\hline Main cooler inlet & 390 & 4.95 & 53.5 \\
\hline Cooling gas compressor inlet & 236 & 4.90 & 56.8 \\
\hline $\begin{array}{l}\text { Cooling gas for coaxial hot } \\
\text { gas duct }\end{array}$ & 300 & 5.12 & 22.9 \\
\hline Sealing gas outlet & 50 & 52.75 & 2.3 \\
\hline
\end{tabular}

Figure 2.6 HHV test circuit and parameters [18]

\subsubsection{Compact Heat Exchanger}

The heat exchangers incorporated in the power conversion system of the HTGR, the recuperator and/or the IHX, need to have a high effectiveness, and superior mechanical characteristics as 
they operate under conditions of high pressure and high temperature. Furthermore, they are required to be as compact as possible to limit their size to enhance the plant layout.

Many ways are used to classify heat exchangers. For example, the fluid types (gas-gas, gasliquid, liquid-liquid), the flow arrangement (counter-flow, cross-flow), surface compactness, the construction type and industry are used.

In a heat exchanger, the heat-exchanger surface (or matrix) is the structure in which heat transfer takes place from one fluid to another fluid. One of the fundamental characteristics of a heatexchanger surface is the surface area per unit of volume occupied by the surface. A "compact heat-exchanger surface" is defined as a surface configuration or matrix having a "large" surface area per unit of volume. Usually, any matrix with an area density greater than $328 \mathrm{~m}^{2} / \mathrm{m}^{3}$ is defined as compact matrix or compact surface [20]. A compact heat exchanger is constructed from compact surfaces.

As discussed in reference [21], a shell-and-tube type heat exchanger is too large to be economic without an extensive materials qualification for HTGR application. Therefore, in this work, the non-tubular compact heat exchanger will be considered as the base design for the recuperator and IHX. Many current technologies of compact heat exchangers are available including plate fin, spiral, microchannels, and plate.

Plate fin heat exchangers (PFHE) have been extensively used in applications such as industrial, natural gas liquefaction, air separation and hydrocarbon separation. The fins are brazed to the parting sheets and then the parting sheets are assembled to form a single block. The blocks are stacked and then the inlet and outlet headers are welded to the blocks to construct a heat exchanger. Numerous fin configurations such as straight fin, straight perforated fin and serrated fin have been developed.

Spiral heat exchangers (SHE) are often used in applications where a phase change occurs. In the SHE, the fundamental part is two metal plates welded together and rolled to form the flow passages.

Microchannel heat exchangers are heat exchangers in which the flow channels are around or less than $1 \mathrm{~mm}$ in diameter. The small channels are manufactured on flat plates by means of technologies such as chemical etching, micromachining or electron discharge machining. A typical microchannel heat exchanger is the printed circuit heat exchanger developed by the Heatric company[22]. In the printed circuit heat exchanger, the plates are stacked and then diffusion bonded. Compared to other type heat exchangers, the microchannel heat exchanger is heavier if the sizes are the same.

Plate heat exchangers (PHE) have been widely used in the applications of chemical, petrochemical, district heating and power industries. A PHE is constructed by the stacking of corrugated plates. Different materials such as aluminum or stainless steel are used for different operating conditions and three technologies such as gaskets, welding and brazing can be used to ensure tightness. The applicable limits for different types of compact heat exchangers are shown 
in Table 2.1[23]. Note that the maximum pressure and maximum temperature cannot be reached simultaneously.

Under HTGR conditions, a high pressure difference is imposed on the recuperator $(>4 \mathrm{MPa})$ and high temperature operation (no lower than $850^{\circ} \mathrm{C}$ ) is required for the IHX. Thus, only welded, brazed or diffusion bonded heat exchangers can be used, as shown in Table 2.2 [23].

Table 2.1 Operating conditions for compact heat exchangers [23]

\begin{tabular}{|c|c|c|c|}
\hline Technology & $\begin{array}{l}\text { Max. pressure } \\
(\mathrm{MPa})\end{array}$ & $\begin{array}{l}\text { Max. temperature } \\
\left({ }^{\circ} \mathrm{C}\right)\end{array}$ & Fouling \\
\hline Stainless steel plate fin heat exchanger & 8 & 650 & No \\
\hline Aluminum plate fin heat exchanger & $8-12$ & $70-200$ & No \\
\hline Ceramic plate fin heat exchanger & 0.4 & 1300 & No \\
\hline Spiral heat exchanger & 3 & 400 & Yes \\
\hline Diffusion bonded heat exchanger & 50 & $800-1000$ & No \\
\hline Brazed plate heat exchanger & 3 & 200 & No \\
\hline Welded plate heat exchanger & $3-4$ & $300-400$ & Yes/no \\
\hline Gasketed plate heat exchanger & $2-2.5$ & $160-200$ & yes \\
\hline
\end{tabular}

Table 2.2 Potentially applicable HX technology for HTGR[23]

\begin{tabular}{|l|c|c|c|}
\hline Technology & Pressure & Effectiveness & Reliability \\
\hline Spiral & $3 \mathrm{MPa}$ & Good & Good \\
\hline Plate-fin & $8 \mathrm{MPa}$ & Very good & Good \\
\hline Welded plates & $3-4 \mathrm{MPa}$ & Good & Good \\
\hline Diffusion bonded & $50 \mathrm{MPa}$ & Good & Very good \\
\hline
\end{tabular}

\subsubsection{Control Methods for Gas Turbine Power Conversion Systems}

The closed cycle provides unique opportunities for power regulation. The closed cycle, helium turbine plant can be designed using the same principles used for closed air turbine plants. Figure 2.7 is a schematic of a regenerated Brayton cycle. The commercial power plant offers the advantage of enabling the power generation to match the required load. Several control methods -- bypass valve control, temperature modulation and inventory control, might be of interest for use in the nuclear gas turbine power conversion system.

1. Bypass valve control

As shown in Figure 2.7, a bypass valve bleeds high-pressure gas to short-circuit the heat source and the turbine. This throttling process is a source of irreversibility and thus reduces the cycle part load efficiency. One part of the high-pressure gas, bypassing the turbine, results in turbine output decrease. At the same time, the cycle pressure ratio is reduced, and thus the mass flowrate through the compressor increases. If the rotational speed remains constant, the velocity triangles for the compressor and turbine are both not in the optimum condition, resulting in a decrease of the cycle efficiency. 
The advantage of bypass valve control is that it can alter the turbine output rapidly to match the load variation. Thus, to achieve fast load change, bypass valve control is usually used in the closed gas turbine system, especially in a large system since the inventory control response is relatively slow. In the event of grid separation, the bypass valve control is always used to prevent the shaft from over-speeding.

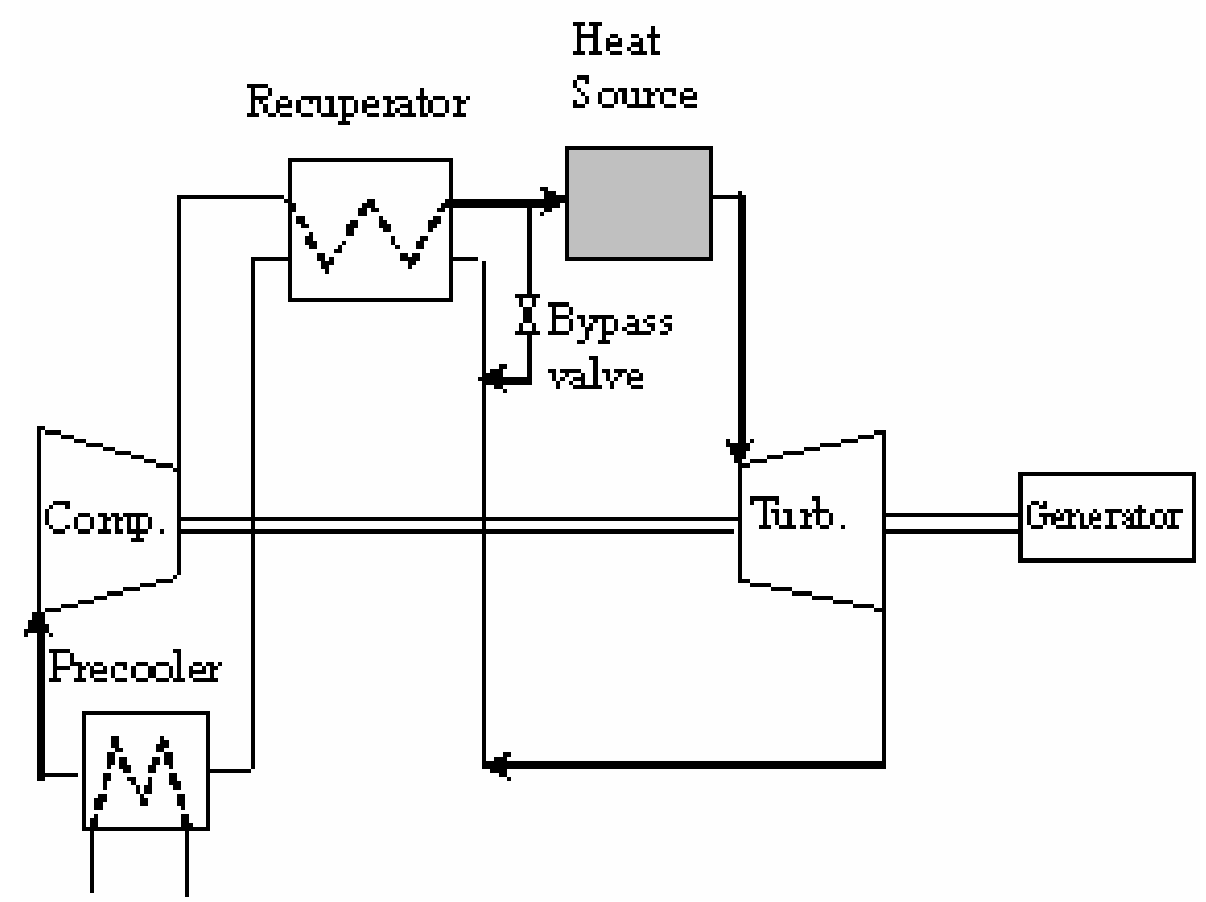

Figure 2.7 Bypass control of a closed Brayton cycle

\section{Temperature modulation}

Decreasing the turbine inlet temperature results in a decrease of the turbine output power and the turbine efficiency, and thus the cycle efficiency. The temperature modulation scheme utilizes this principle.

For the HTGR gas turbine plant, adjusting the reactor power can alter the core outlet temperature, and thus the gas turbine inlet temperature. Due to the large thermal inertia of the reactor core, the change of the core outlet temperature is relatively slow. Accordingly, temperature modulation is not suitable for fast power control.

\section{Inventory control}

As shown in Figure 2.8, the inventory of the working fluid in the closed power system is controlled by moving mass to or from a storage vessel. A compressor may be used to pump the 
working fluid from the system to the storage vessel as the load decreases although the $\Delta \mathrm{P}$ across the compressor can also be used. The reduced mass inventory in the system results in a smaller mass flow rate, and thus a lower turbine power output. When the load increases, the working fluid in the storage vessel is fed back to the system. To minimize the heat energy moving from the system to the storage vessel, the working fluid can be removed from a point with the lowest temperature of the cycle. With the reduced mass flowrate, the temperatures and pressure ratio of the cycle remain constant, thus the thermodynamic cycle is unaltered.

When the temperatures remain constant, the sonic speed of the working gas does not change as the mass flowrate decreases. The blading and flow passage geometries fix the Mach number. This implies that the flow velocities along the cycle are constant and thus the mass flowrate is proportional to the flow density. Also, the mass flowrate is proportional to the pressure level. The T-s diagrams for part and full power are shown in Figure 2.9[23].

As the pressure level decreases, the pressure losses will be slightly changed because the decrease in density also causes a decrease in the Reynolds number. The effect is that the cycle pressure ratio shifts from the design value and thus the cycle efficiency decreases slightly.

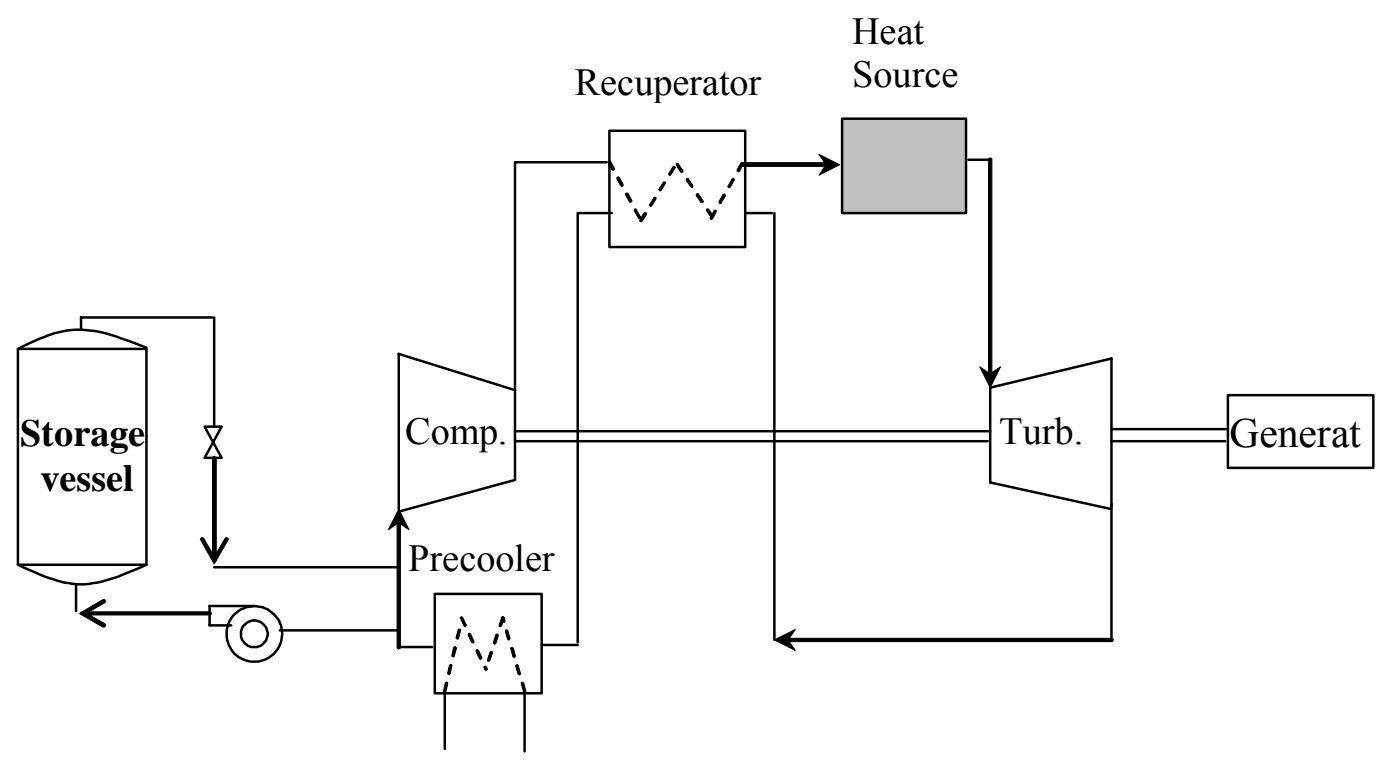

Figure 2.8 Closed cycle with inventory control

Figure 2.10 shows the cycle efficiency under different control methods. We can see that the cycle efficiency at partial load remains high by using inventory control while the bypass valve control and temperature modulation degrade the cycle performance.

\subsection{Advanced Gas Cooled Reactor Design Requirements}


The HTGR gas turbine plant is being developed as a Generation IV nuclear energy system which offers advantages in the areas of economic competitiveness, safety and reliability. The MPBR promises a number of significant advantages over conventional commercial water-cooled technology. First, by fully using the high gas temperature, the MPBR provides a thermal efficiency approaching $45 \%$. Higher efficiency leads to improved economics. The MPBR will be a demonstrably safe nuclear plant system. This implies that the system will be designed such that any postulate accidents will not result in fuel melt. Thus, no fuel damage and release of radioactivity to the environment will occur. This inherent safety is due to the fact that the core will be designed with a negative temperature coefficient of reactivity and the decay heat can be removed to the ground by a passive heat transfer mechanism. The passive heat transfer mechanism includes conduction and natural convection. Since the coolant is inert helium in the MPBR, corrosion of the components is not a concern so that the cost for replacement of the degraded components caused by corrosion such as in water-cooled reactors is avoided. This simplifies operation and maintenance and thus improves the economics.

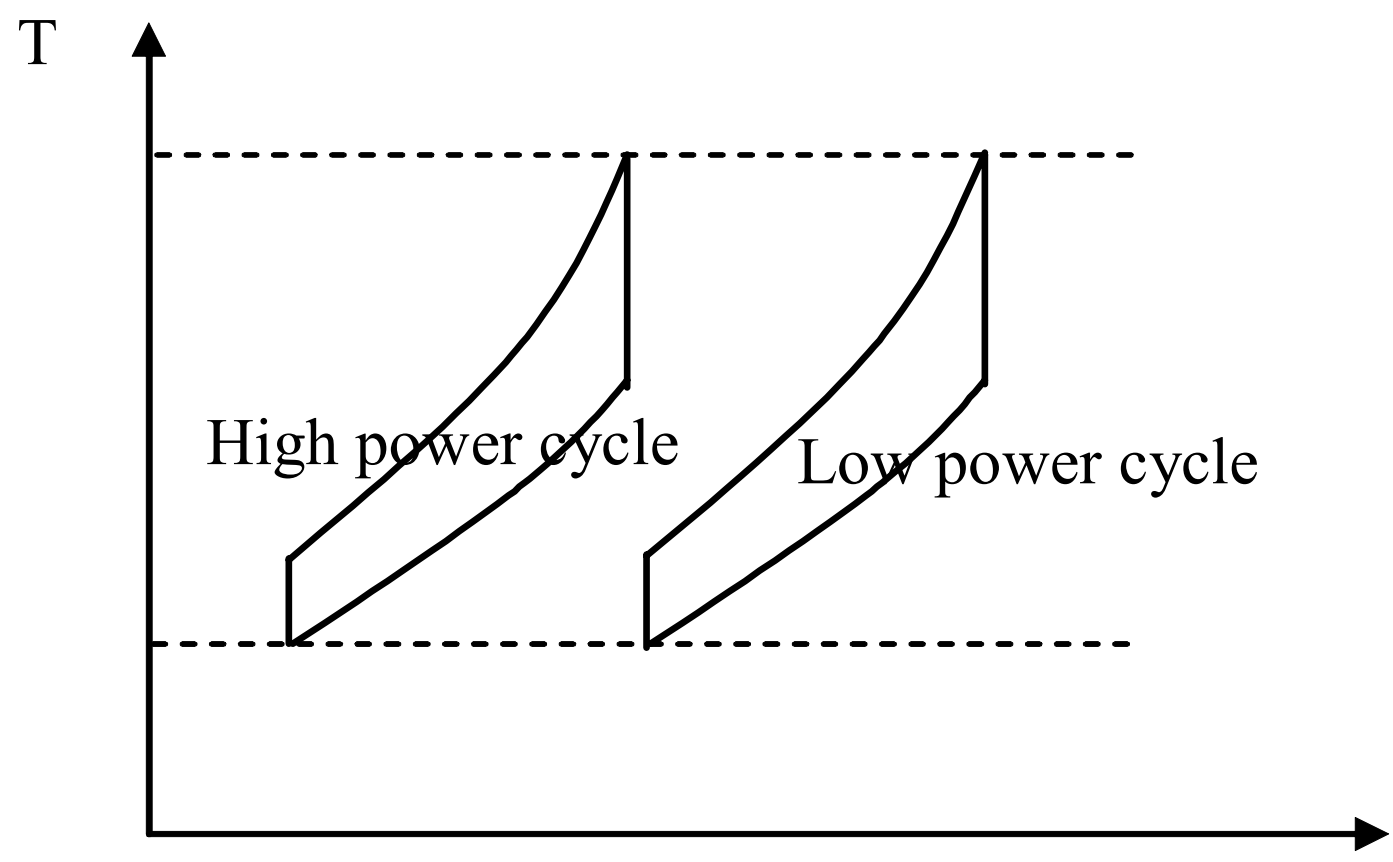

Figure 2.9 T-s diagram for part power and full power for the inventory controlled closed Brayton cycle [24]

Overall, the objective of the MPBR is that it's economics can compete with natural gas. With regard to the balance of plant design, the requirements can be summarized as follows:

(a) High efficiency over a broad operating range;

(b) Load following;

(c) Low capital cost;

(d) Constructability; 
(e) Modularity;

(f) Transportability;

(g) Code compliance.

PART POWER PERFORMANCE

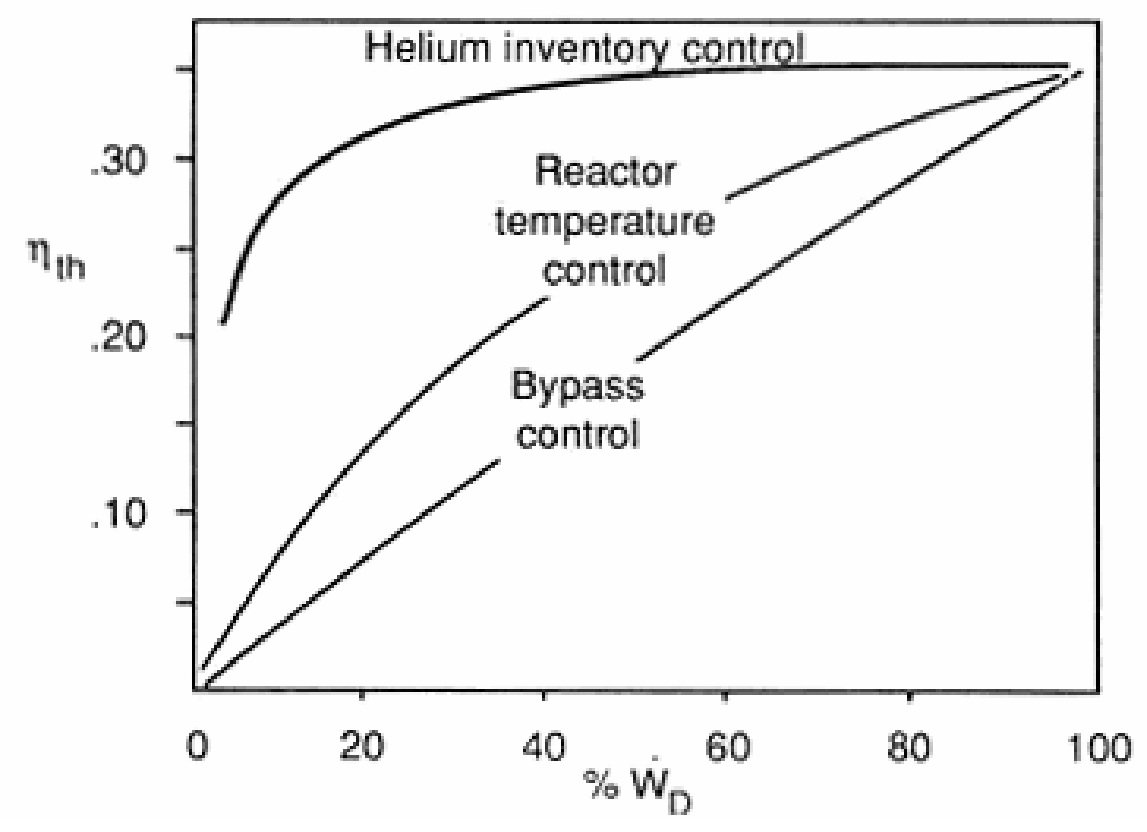

Figure 2.10 Performance of inventory, bypass and temperature control [24]

These goals will require that the design provides high efficiency during full power operation and also high efficiency during partial power operation. From a control point of view, the plant must be capable of meeting the utility requirement for load following as an advanced nuclear system. Considering the components in the power conversion system, the constructability, complying with current codes and with no significant R\&D effort need to be considered in making design decisions. Modularity is a key consideration for component design so that the failed module can be unplugged and replaced quickly by a spare one. The component modules are manufactured off-site and transported to the construction site by flat bed truck or rail car.

\subsection{Overall Development Path}

The overall development path followed in this project was to first build a "reference" design which satisfies all the codes and standards. Based on the "reference" design, a steady state model was developed and the key limitations were identified. The steady state model was used to calculate the plant thermal efficiency, the pressure ratio of the power conversion system, and other parameters such as temperatures and pressures in the cycle. Then key issues were identified. The questions include those related to the feasibility of the IHX and recuperator, the helium gas turbine and compressor, system control and the consequence of indirect cycle choice. To design the control system for the power conversion system, a dynamic model was then 
developed. The dynamic model integrates the reactor core and the power conversion system and incorporates the control schemes. Then a path was quantified to remove limitations of the "reference" design. Meanwhile, the steady state model and the dynamic model were used to optimize the design. To satisfy all the requirements and limitations, design iterations and compromises were sometimes required. Finally, an advanced design was developed.

\section{References: Section 2}

[1] Gilbert Melese, Robert Katz, “ Thermal and flow design of helium-cooled reactors”, American Nuclear Society, 1984.

[2] H. L. Brey, "Developmental history of the gas turbine modular high temperature reactor", IAEA TCM on gas turbine power conversion system for modular HTGRs, Nov. 14-16, 2000, Palo Alto, California.

[3] http://www.ga.com/gtmhr/gtmhr1.html.

[4] R. A. Moore, et al., "HTGR experience, programs, and future applications", Nuclear engineering and design 72(1982), pp. 153-174.

[5] H. L. Brey, "Fort St. Vrain circulator operating experience", IAEA Specialists' meeting on gas-cooled reactor coolant circulator and blower technology. San Diego, CA (USA), 30 Nov. - 2 Dec. 1987.

[6] http://www.jaeri.go.jp/english/temp/temp.html.

[7] http://www.pbmr.co.za/.

[8] International Atomic Energy Agency, "Gas cooled reactor design and safety", Technical report series No. 312, Vienna, 1990.

[9] Y. Xu, "Chinese point and status", Proceedings of the conference on high temperature reactors, Petten, Netherlands, April 22-24, 2002.

[10] Yuanhui Xu, Kaifen Zuo, "Overview of the $10 \mathrm{MW}$ high temperature reactor - test module project”, Nuclear engineering and design 218(2002),13-23.

[11] M. P. LaBar, "The Gas Turbine - Modular Helium Reactor: A promising option for near term deployment", General Atomics report No. GA-A23952.

[12] A. Kiryushin, N. G. Kodochigov, " GT-MHR project”, Proceedings of the conference on high temperature reactors, Petten, Netherlands, April 22-24, 2002.

[13] K. N. Kumar, A. Tourlidakis, P.Pilidis, "Performance review: PBMR closed cycle gas turbine power plant", IAEA TCM gas turbine power conversion systems for modular HTGRs", November 14-16, 2000 in Palo Alto, California.

[14] Y. Q. Wu, X. Z. Diao, etc, "Design and tests for the HTR-10 control rod system", Nuclear engineering and design 218 (2002) 147-154.

[15] H. Z. Zhou, Z. Y. Huang, X. Z. Diao, "Design and verification test of the small absorber ball system of the HTR-10”, Nuclear engineering and design 218 (2002) 155-162.

[16] Xinglong Yan, "Dynamic analysis and control system design for an advanced nuclear gas turbine power plant", MIT PhD thesis, 1990.

[17] Tony Giampaolo, "The gas turbine handbook: principles and practices", Fairmont press, 1997.

[18] I. A. Weisbrodt, "Summary report on technical experiences from high-temperature helium turbomachinery testing in germany", International Atomic Energy Agency, Vienna, Nov. 1994.

[19] K. Bammert, "Operation and control of the 50-MW closed-cycle helium turbine Oberhausen”, ASME paper 74-GT-13. 
[20] Northern Research and Engineering Corporation, "The design and performance analysis of compact heat exchangers", 1965.

[21] Y. Muto, K. Hada, H. Koikegami and H. Kisamor, "Design of intermediate heat exchanger for the HTGR-closed cycle gas turbine power generation system", JAERI-Tech, 96-042, October, 1996.

[22] http://www.heatric.com.

[23] B. Thonon, E. Breuil, "Compact heat exchangers technologies for the HTRs recuperator application", IAEA TCM gas turbine power conversion systems for modular HTGRs", November 14-16, 2000 in Palo Alto, California.

[24] Reiner Decher, "Energy conversion systems, flow physics and engineering", Oxford university press, 1994. 


\section{Results: Gas Turbine Power Conversion System Design Considerations}

\subsection{Introduction}

High core outlet temperature is the main advantage of the high temperature gas cooled reactor. To take full advantage of the high outlet temperature, a new power conversion system, differing from the Rankine cycle, must be used.

The general design considerations, such as working fluid and cycle options, and the design constraints will be addressed. The advantages and disadvantages for cycle variations will be demonstrated. The feasibility of the compact heat exchangers and helium gas turbomachinery will also be investigated.

\subsection{General Design Considerations}

Figure 3.1 shows a decision tree which depicts the decision making path to narrow the choice of system cycle and the numerical analysis. It provides an outline of the issues which needed to be addressed. The trade-offs were considered based on technical characteristics and economics.

One of the main advantages for the high temperature gas cooled reactor is that it can provide a high core outlet temperature $\left(>850^{\circ} \mathrm{C}\right)$. When steam is used in a Rankine cycle, its temperature is limited by the high pressure imposed by the pressure-temperature relationship along the saturation line. One can superheat the steam but this complicates the plant layout. A practical temperature limit is around $300-400^{\circ} \mathrm{C}$ for the Rankine cycle. To take advantage of the high core outlet temperature of the HTGR, a Brayton cycle is preferred.

Other design considerations involve four aspects: closed cycle versus open cycle, direct cycle versus indirect cycle, working fluid choice and system pressure. The following sections describe them.

\section{- Closed Cycle vs. Open Cycle}

The combustion gas turbine cycle usually adopts an open cycle, in which the system inlet pressure is atmospheric and air is the working fluid. When utilizing nuclear energy as the heat source, radioactivity is one of the main considerations in power conversion system design. In this case, the direct, open cycle is inadvisable. By using an intermediate heat exchanger (IHX) to separate the nuclear system (primary system) and the "secondary" system, one obtains the so called indirect cycle. However, using an open cycle in the secondary system makes the inlet pressure in the power conversion system equal to atmospheric pressure, about $0.1 \mathrm{MPa}$. This pressure level would lead to an IHX having a large volume. An approximate estimation shows that the volume of the IHX in an open air cycle is a factor of 15 larger than that in a helium closed cycle and a factor of 3 larger than that in an air closed cycle[1]. Therefore, in terms of compactness and capital cost, a closed cycle is favored. 
(e) Cycle variations

(f) Arrangement of power

conversion unit

(e) Working fluid choice

(d) Coupling with reactor

(c) Coupling with

ervironment

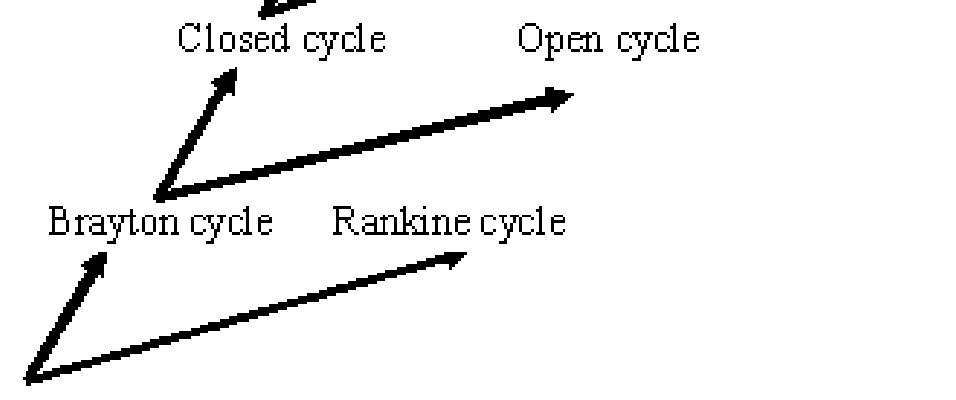

Indirect cycle Jirect cycle
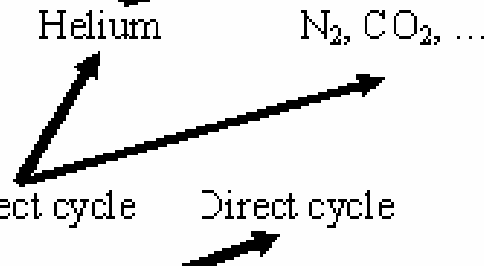

(b) Thermodynamic

cycle

(a) Heat source

Figure 3.1 Decision tree for specification of power conversion unit

\section{- Direct Cycle vs. Indirect Cycle}

There are two types of closed cycles - direct cycle and indirect. The direct cycle circulates working fluid exiting from the reactor core directly to the power conversion unit and the working fluid exhausting from there back to the core, while the indirect cycle utilizes an IHX to separate the primary system and the power conversion unit. The IHX transfers the thermal energy from the primary system to the working fluid of the gas turbo-machines, which convert the thermal energy to mechanical energy. The mechanical energy is then transformed into electrical energy by the generator. The direct cycle has the advantages of higher efficiency (higher turbine inlet temperature) and less components (without the IHX). Its disadvantage is that the power conversion unit is contaminated, which results in higher cost for maintenance and higher component costs due to their adaptation to nuclear standards. Also, there is potential damage to the primary boundary by turbo-machine failures, i.e., turbine blade failure. The indirect cycle has a lower efficiency compared with the direct cycle because the IHX results in a temperature drop from the reactor outlet to the turbine inlet. The elimination of contamination in the power 
conversion unit in the indirect cycle makes maintenance simple and allows components to be built to non-nuclear standards, thus cost less. Since there are two circuits in the indirect cycle, a depressurization accident occurring in one circuit imposes a high pressure differential on the IHX, and could cause IHX failure. These great care must be taken to include appropriate safety systems to prevent this from happening. Table 3.1 summarizes the pro's and con's for the indirect cycle. The indirect cycle is chosen for the MPBR.

Table 3.1 Summarization of the pro's and con's for indirect cycle

\begin{tabular}{|l|l|l|}
\hline & \multicolumn{1}{|c|}{ Advantages } & \multicolumn{1}{c|}{ Disadvantages } \\
\hline 1 & Ease and less cost of maintenance & Slightly lower cycle efficiency \\
\hline 2 & Avoidance of the "missile" accident & One more component cost: an IHX \\
\hline 3 & $\begin{array}{l}\text { Less expensive components for power } \\
\text { conversion unit: do not need to build to } \\
\text { nuclear standards }\end{array}$ & $\begin{array}{l}\text { Higher complexity: one more } \\
\text { component - an IHX }\end{array}$ \\
\hline 4 & Less potential for water ingress accident & IHX "operating curve" required \\
\hline
\end{tabular}

\section{- Working Fluid Choice}

The choice of working fluid for the nuclear gas turbine cycle considerably affects the cycle efficiency and the system compactness. Of primary importance in selecting the medium for the working fluid is material compatibility, e.g., minimum corrosion and chemical reaction with other component materials. High thermal capacity and radiation stability are also important characteristics that need to be considered.

Helium is an inert gas. It is nuclear transparent, implying that it does not absorb neutrons, thereby it does not become radioactive. From experience in energy production, the Light Water Reactor (LWR) and fossil industries have a long history of corrosion related component reliability problems. Helium is chemically inert and does not inflict a chemical corrosion on the components, such as turbines, and thus does not lead to component degradation.

In Table 3.2, the properties of helium and air are compared at $1000 \mathrm{~K}$ and $3 \mathrm{MPa}$. One can note that the specific heat and thermal conductivity of helium are both about five times that of air. Numerical comparisons have been made for helium and air in references[2, 3]. For similar pressure losses the flow velocity of helium can be double that of air $[2,3]$ and the heat transfer coefficient for helium is almost twice that for air [2]. This implies that, using helium, the heat exchanger only needs the half heat transfer area for the same temperature difference, or that higher effectiveness can be reached economically. Considering a unit mass of working fluid passing through a turbine, because the specific heat of helium is 5 times that of air, the helium enthalpy change is also 5 times that of air when the temperature differences are the same. One might expect that the stages of a helium gas turbine are also 5 times that of an air gas turbine. Actually, it is not so. The sonic velocity in helium is much higher than that in air, there is virtually no Mach number limitation on flow velocity and peripheral speed in turbomachinary design when using helium as the working fluid. The number of stages will not increase significantly for helium gas turbo-machinery. This is further amplified s in Appendix B. 
Table 3.2 Properties of helium and air at $1000 \mathrm{~K}$ and $3 \mathrm{MPa}$

\begin{tabular}{|l|c|c|}
\hline Property & Air & Helium \\
\hline Molecular weight & 28.9 & 4 \\
\hline$\gamma$ & 1.36 & 1.667 \\
\hline $\mathrm{Cp}(\mathrm{J} / \mathrm{kg} \mathrm{K})$ & 1142 & 5193 \\
\hline Thermal conductivity $(\mathrm{W} / \mathrm{m} \mathrm{K})$ & 0.068 & 0.36 \\
\hline Kinetic viscosity $\left(\mathrm{m}^{2} / \mathrm{sec}\right)$ & 3.4 & 28 \\
\hline Sonic velocity $(\mathrm{m} / \mathrm{s})$ & 628.0 & 1861.8 \\
\hline
\end{tabular}

When using helium as working fluid, the disadvantage is that it is a lighter gas, thus is prone to leaks. Reliable sealing, including advanced dry gas sealing technology, for the system at the high pressure required is the main consideration for large power plant operation. In the MPBR design, helium is both used in the primary system and the power conversion unit. The pressure in the power conversion unit is higher than the primary system pressure, thereby providing resistance for release of radioactivity to the power conversion unit.

Ordinary $\mathrm{CO}_{2}$ has been used in the primary system in Magnox Reactors (the United Kingdom) and Supercritical $\mathrm{CO}_{2}\left(\mathrm{SCO}_{2}\right)$ is being studied in another project at MIT[4] as the working fluid. Corrosion is the main challenge if using $\mathrm{CO}_{2}$ or $\mathrm{SCO}_{2}$ as the working fluid and leakage is also a concern for $\mathrm{SCO}_{2}$ since a very high system pressure is used, i.e. $20 \mathrm{MPa}$.

\section{- System Pressure for Closed Cycle}

The system pressure level does not affect the efficiency of a closed cycle using helium because the efficiency depends on cycle pressure ratio and the corresponding temperature ratio. However, the pressure level affects the dimensions of the components in the cycle, i.e., the piping, turbomachinery, heat exchangers and especially the reactor pressure vessel. Also, the choice of the piping wall thickness depends on the pressure (at fixed temperature). Table 3.3 shows the pipe inner diameter for different pressures with a fixed helium mass flowrate of $126.7 \mathrm{~kg} / \mathrm{s}$, a fixed temperature of $30^{\circ} \mathrm{C}$ and an assumed flow velocity of $120 \mathrm{~m} / \mathrm{s}$. Increasing the pressure allows a reduction in pipe diameter. On the contrary, the wall thickness needs to be increased to ensure the wall stress imposed by the pressure is low enough. In the MPBR design, the maximum pressure is taken as $8.0 \mathrm{MPa}$.

Table 3.3 Pipe inner diameter required for different pressures at fixed mass flowrate $126.7 \mathrm{~kg} / \mathrm{s}$, fixed temperature $30^{\circ} \mathrm{C}$ and fixed flow velocity $120 \mathrm{~m} / \mathrm{s}$.

\begin{tabular}{|c|c|}
\hline Pressure $(\mathrm{MPa})$ & Pipe inner diameter $(\mathrm{m})$ \\
\hline 1.0 & 0.922 \\
\hline 2.0 & 0.654 \\
\hline
\end{tabular}




\subsection{Design Constraints}

The design developed in this project complies with existing codes and standards in the US. The nuclear island of the MPBR uses a nuclear reactor design similar to that developed in South Africa. The pressure vessel in this design uses A508/A533 class steel as the reactor pressure vessel (RPV) with an upper temperature limitation of $375^{\circ} \mathrm{C}[5]$. This restriction requires a separate cooling system for the hot section components. The application of the ASME code Section III (Class I) results in a temperature limit of $427^{\circ} \mathrm{C}$ for the IHX pressure vessel and all of the Class I boundary. To satisfy the code requirements and, at the same time, to allow higher turbine inlet temperatures, component cooling and/or thermal insulation are used where required. Component cooling, since it requires the diversion of gas that could be used in power conversion, will result in an efficiency penalty and is thus only used where necessary. A significant advantage of the indirect cycle is the limiting of the Section III Class I boundary requirements to components and piping from the reactor to the IHX. ASME code Section VIII requirements will be used as the basis for the components in the power conversion unit beyond the IHX. Section VIII allows temperatures up to $898^{\circ} \mathrm{C}$. In the power conversion unit, the temperatures are usually below this limitation. However, due to concerns for differential thermal expansion, cooling is used for all of the turbine casings and interconnecting piping.

Current open cycle gas turbine technology allows for power levels up to 200MW and beyond. However, experience for design and operation of closed cycle helium turbomachinery is limited. As described in Section 2, the largest capacity helium turbine built to date is the 50MWe unit in Oberhausen Germany. After detailed discussions with the manufacturers for turbomachinery, it was determined that the current helium turbine technology will allow powers no higher than 52MW without significant extensions of technology with associated large R\&D costs.

Notwithstanding the claims by many that larger turbines can be easily built, when it comes to an actual commitment by a manufacturer to a design with a guaranteed efficiency, the reality of the difficulty, and associated risk of assuming larger turbines becomes apparent.

With respect to piping sizes, higher velocity results in smaller piping diameters which will reduce costs. Additionally, minimization of diffuser losses in the turbomachinery, also argues for a helium velocity as high as possible. However, when considering the potential erosion on piping, in the current design $120 \mathrm{~m} / \mathrm{s}$ was chosen as the piping helium maximum velocity.

With respect to heat exchanger design, current technology limits the maximum size for compact, plate fin or printed circuit designs.

An additional restriction was applied with respect to plant layout. The design layout makes use of a horizontal configuration. This configuration was chosen for ease of maintenance and bearing design. The design calls for the use of magnetic bearings. However, while magnetic bearing design technology has greatly matured over the past 10 years, the majority of magnetic bearing experience has been with horizontal configurations. Additionally, while magnetic bearings have been built which will support the weight of a single shaft design, these bearings have been limited to rotational speeds of several hundred RPM, not the several tens of thousand RPM anticipated for single shaft designs. Thus, again, we have chosen to use technology with maximum industrial experience and minimum extension of technology. 
The last constraint concerns the use of modularity. In our design we advocate the maximum use of modular designs for components. This places constraints on the maximum sizes for individual components such as heat exchangers and requires the use of individual modules as building blocks for the IHX, recuperator, and precoolers.

\subsection{Configuration Consideration}

For the gas turbine cycle, the shaft arrangement needs to be considered. In order to improve the cycle efficiency, some cycle variations, i.e., recuperated and/or intercooled cycles, are possible options.

\section{- Shaft arrangement}

The configuration of the power conversion unit should be determined after consideration of both the technology of gas turbo-machines and its layout. So far, there are two options for turbomachine configuration: (a) Single-shaft arrangement, (b) Multi-shaft arrangement.

A turbine which drives compressors and the generator through a single shaft, shown in Figure 3.2 , is called a single-shaft arrangement. In this arrangement, usually one or two compressors might be used. If more than one compressor, they are mechanically connected.

The electric grid synchronous frequency is $60 \mathrm{~Hz}$ and thus the generator rotational speed must be $3600 \mathrm{rpm}$ or a multiple of this if not using a frequency converter. To allow turbines and compressors to run at high rotational speed, which makes their efficiencies high and their dimensions small, a speed reducing gearbox or a frequency converter are required. The gearbox and frequency converter both cause energy loss, especially during part load operation, and thus result in a decrease of cycle efficiency. The maximum practical power output of a viable gearbox is around $80 \mathrm{MW}$. The single-shaft arrangement suits operating at a fixed speed and fixed load condition such as meeting the base load power requirement. In this case, the loadfollowing operation capability, for example, the rapid accommodation to changes of load and rotational speed, and efficiency at part load, is not important. The advantage of the single-shaft arrangement is its reduced danger of over-speeding in the event of total load rejection due to the inherent high inertia caused by the drag of the compressors. It should be noted that as the power level of the plane increases, the optimum rotating speed of the turbo-machinery decreases until, if power outputs approaching 400MW can be achieved, a speed of about 3600 RPM can be used. This would allow for the elimination of the gear box and/or frequency conversion systems. However, at these power levels passive cooling during an accident may become problematic.

To improve the gas turbine cycle efficiency, an axial compressor is preferred since it has higher efficiency and smaller dimensions in comparison with the centrifugal type. However, the axial type is more prone to instability when operating at conditions widely shifted from the design point. This is especially true for the compressor. When such a compressor operates at a rotational speed much below the design speed, the gas density in the last few stages is much too low, the axial flow velocity becomes excessive, and thus forces the first stage to be operated at stall[2,6]. The unstable operation, which causes violent aerodynamic vibration, is likely to occur when a gas turbine is started up or operated at low power. 
A multi-shaft arrangement, typically a three-shaft arrangement shown in Figure 3.3, significantly improves the gas turbine stability due to expanding the compressors stable operating range [7]. Taking the three-shaft arrangement as an example, it divides the compression process into two steps. These two steps are mechanically separated, allowing each section to run at different speeds. The compressor in each section requires its own driving turbine. As shown in Figure 3.3, the high-pressure turbine drives the high-pressure compressor and the low-pressure turbine drives the low-pressure compressor. A mechanically independent (or free) power turbine couples to a generator. The three-shaft arrangement exhibits improved operational stability when the gas turbine operates at part load. The trade is the cost and complexity for the separate shaft system versus the single-shaft arrangement. Although many believe that the three-shaft arrangement is more expensive, designers disagree with about this issue[6].

After detailed discussions with the turbomachinery manufacturers, i.e., Dresser-Rand, ElliottEbara and Siemens, it was determined that the current helium turbine technology will allow powers no higher than 52.0MW (70kHP) for a single body without significant extensions of technology with associated large $R \& D$ costs. All of the manufacturers prefer direct-drive rather than gearboxes at this power level due to cost and maintenance considerations. The three-shaft arrangement permits the power for both the high-pressure turbine and the low-pressure turbine to be around 50MW. For the above reasons, a three-shaft arrangement is adopted in the MPBR design and magnetic bearings are expected to be used. The shafts are all horizontal in layout.

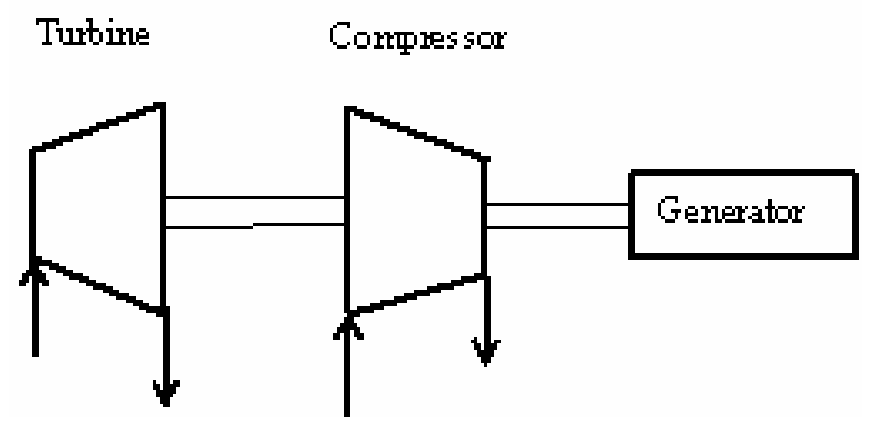

Figure 3.2 Single-shaft arrangement

\section{- Recuperation}

The Brayton cycle with low pressure ratio has a high temperature turbine exhaust flow. The temperature difference between the turbine exhaust and the compressor discharge is large. If a recuperator is employed, the remaining energy of the turbine exhaust is transferred to preheat the gas at core inlet (for direct cycle) or the gas at the secondary inlet of the IHX (for the indirect cycle), and the cycle efficiency can be improved significantly. For the recuperated cycle, a recuperator with high performance is required. 


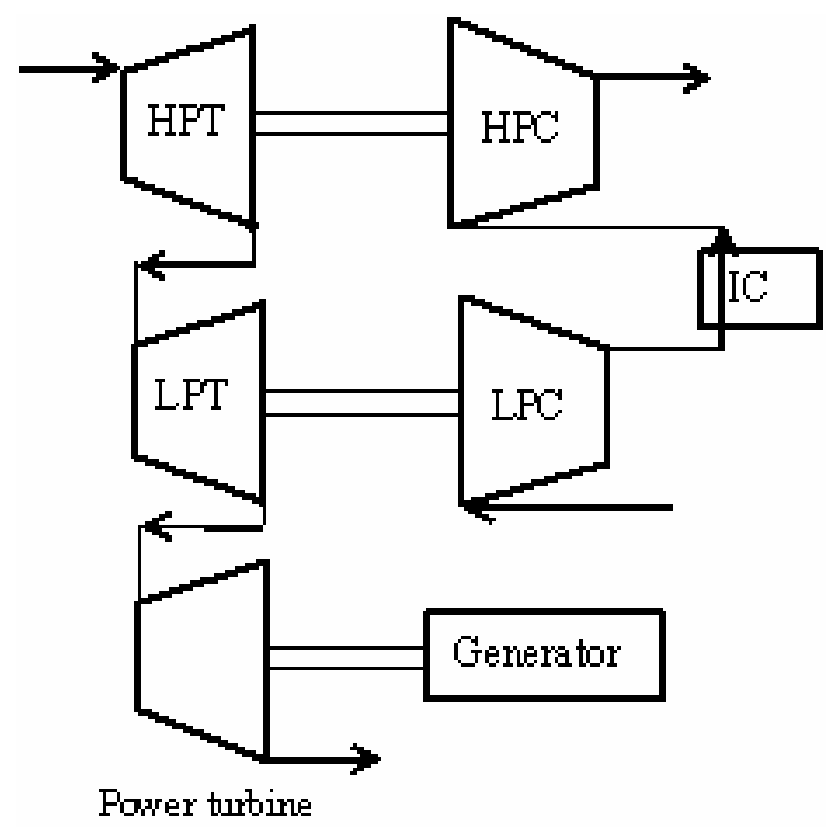

Figure 3.3 Three-shaft arrangement

\section{- Intercooling}

To generate net power output with a closed gas turbine cycle, the power produced by the turbine must be larger than the power consumed by a compressor with an identical pressure ratio. Decreasing the inlet temperature decreases the compressor power at the same pressure ratio. Thus, intercooling can be used to improve the cycle efficiency. The compression process is implemented using several compressors, in which the inlet helium is cooled by a helium/water heat exchanger individually. This results in a significant reduction of the compressor power required to realize the compression task, and thus an increase of the cycle efficiency. The downside of adopting intercooling is increasing the cycle complexity and the requirements for cooling water.

\subsection{Compact Heat Exchanger and Helium Gas Turbo-machinery Design Considerations}

The feasibility of high performance heat exchangers, operated at high temperature and high pressure, and high efficiency helium gas turbines and compressors is vital to implement the gas turbine cycle for the modular pebble bed reactor. Considering the size, weight, and layout, the modules of components should be transported to the site as a unit.

The following describes compact heat exchanger and helium gas turbine design considerations.

\section{- Compact heat exchanger design considerations}


With the pebble bed reactor, the outlet temperature can reach $900^{\circ} \mathrm{C}$, which is the working condition for the IHX if using an indirect cycle. For the recuperator, the hot inlet temperature is around $500^{\circ} \mathrm{C}$ and the pressure difference can be around 5.3MPa. Graphite particles in the primary system can increase the risk of fouling of the primary channels of the IHX. Also, the helium impurity content $\left(\mathrm{H}_{2} \mathrm{O}, \mathrm{H}_{2}, \mathrm{CO}\right.$, etc.) can lead to a risk of corrosion for the heat exchanger channels. With respect to the geometry, a modular concept is proposed which benefits maintenance - simply removing the leaky module and plugging in a spare one. The heat exchanger effectiveness must be high. For the IHX, the effectiveness must be no lower than $90 \%$, and for the recuperator, $95 \%$. To limit the size, compact heat exchangers are the most attractive solution to the design of both the IHX and recuperator.

As described in Section 2.3.2, the criteria for the selection of compact heat exchangers are based on the operating conditions and other features such as weight, cost, compactness, reliability, corrosion and fouling. For the MPBR, two types of compact heat exchanger technology Printed Circuit Heat Exchangers (PCHE) and Plate Fin Heat Exchangers (PFHX) are considered for the IHX and recuperator.

The PCHE was developed by Heatric of Dorset England, which is a division of the Meggitt Corporation. The printed circuit concept makes use of chemically etched flow channels on a plate in a predetermined flow pattern as shown in Figure 3.4. Then the plates are stacked together and diffusion bonded as shown in Figures 3.5, 3.6 and 3.7. There are over 400 units operating worldwide for a wide of range of application up to $500 \mathrm{bar}$ and $800^{\circ} \mathrm{C}$. Units have been in service more than 12 years[7].

Plate fin heat exchangers have been widely used as recuperators in the field of power generation. Ingersoll Rand Energy Systems (IRES), Portsmouth, NH has developed a plate fin heat exchanger that is well suited to the IHX and recuperator applications. The IRES PFHX is manufactured through a patented approach in which the folded fins are brazed to the stamped plates to form the unit-cells as shown in Figure 3.8. The unit-cells are then stacked to form the totally welded pressure boundary as shown in Figure 3.9. The configuration is specifically designed to accommodate substantial thermal strain and therefore to tolerate the severe temperature gradients encountered during transient operations.

The Concepts-NREC company has made thermal designs with PCHX and PFHX configurations for the IHX and recuperator for this project. The design results are described in detail in Appendix A. 


\section{Plates}

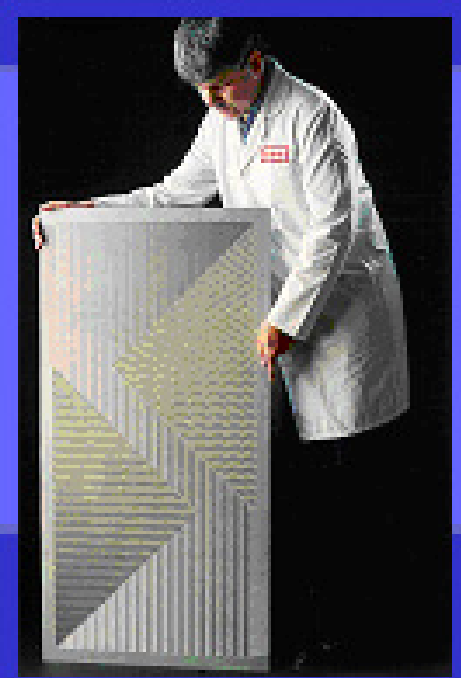

Typically $1.6 \mathrm{~mm}$ thick, $600 \times 1200 \mathrm{~mm}$

- Channels produced by chemical etching

- channels have seinicircular profile with $2 \mathrm{~min}$ diameter

Figure 3.4 Printed circuit plate [7]

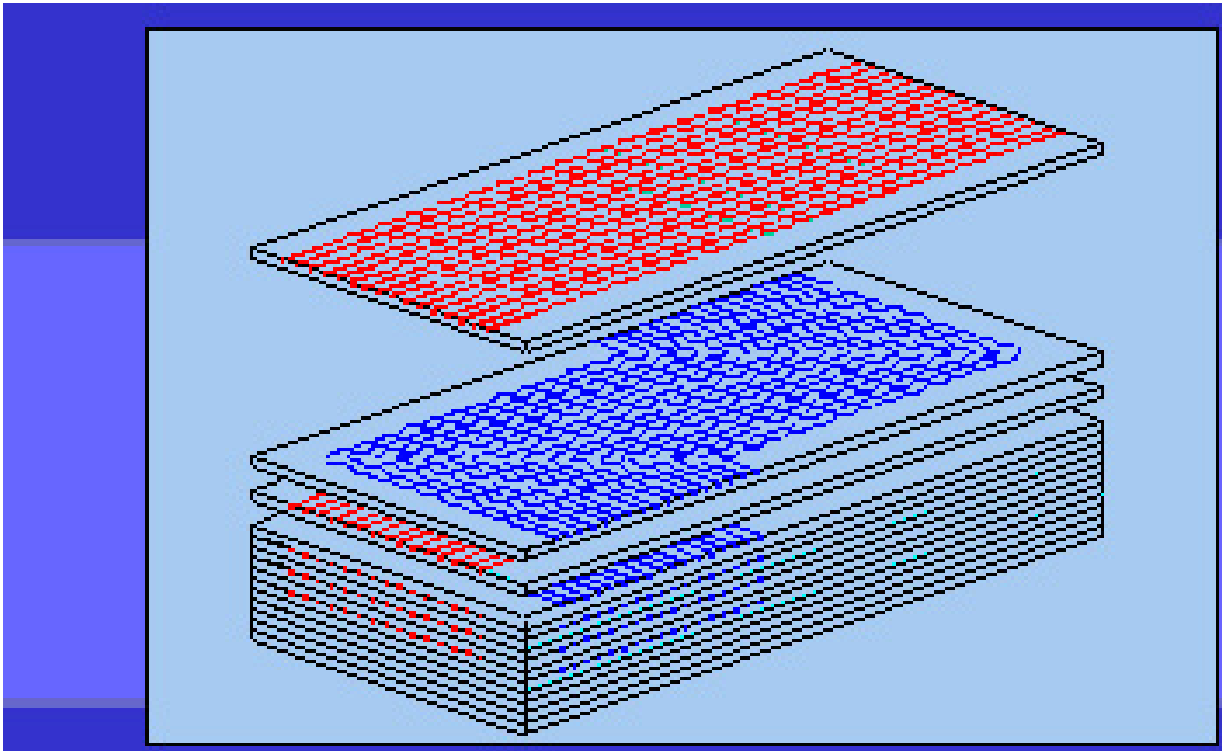

\section{Plate stacking prior to diffusion bonding}

Figure 3.5 Stacking of printed circuit plates [7] 


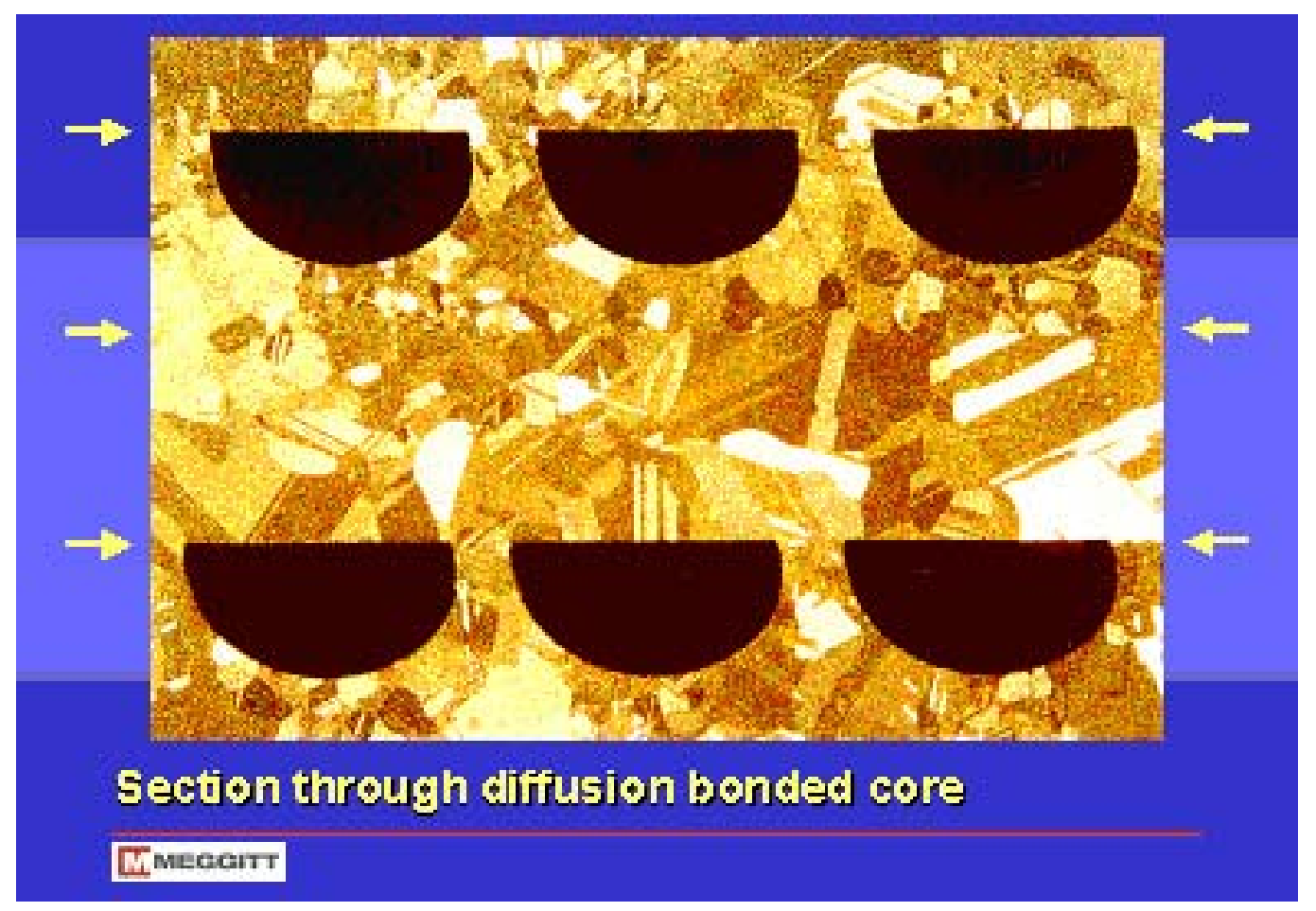

Figure 3.6 Detail of bonded printed circuit plates [7]

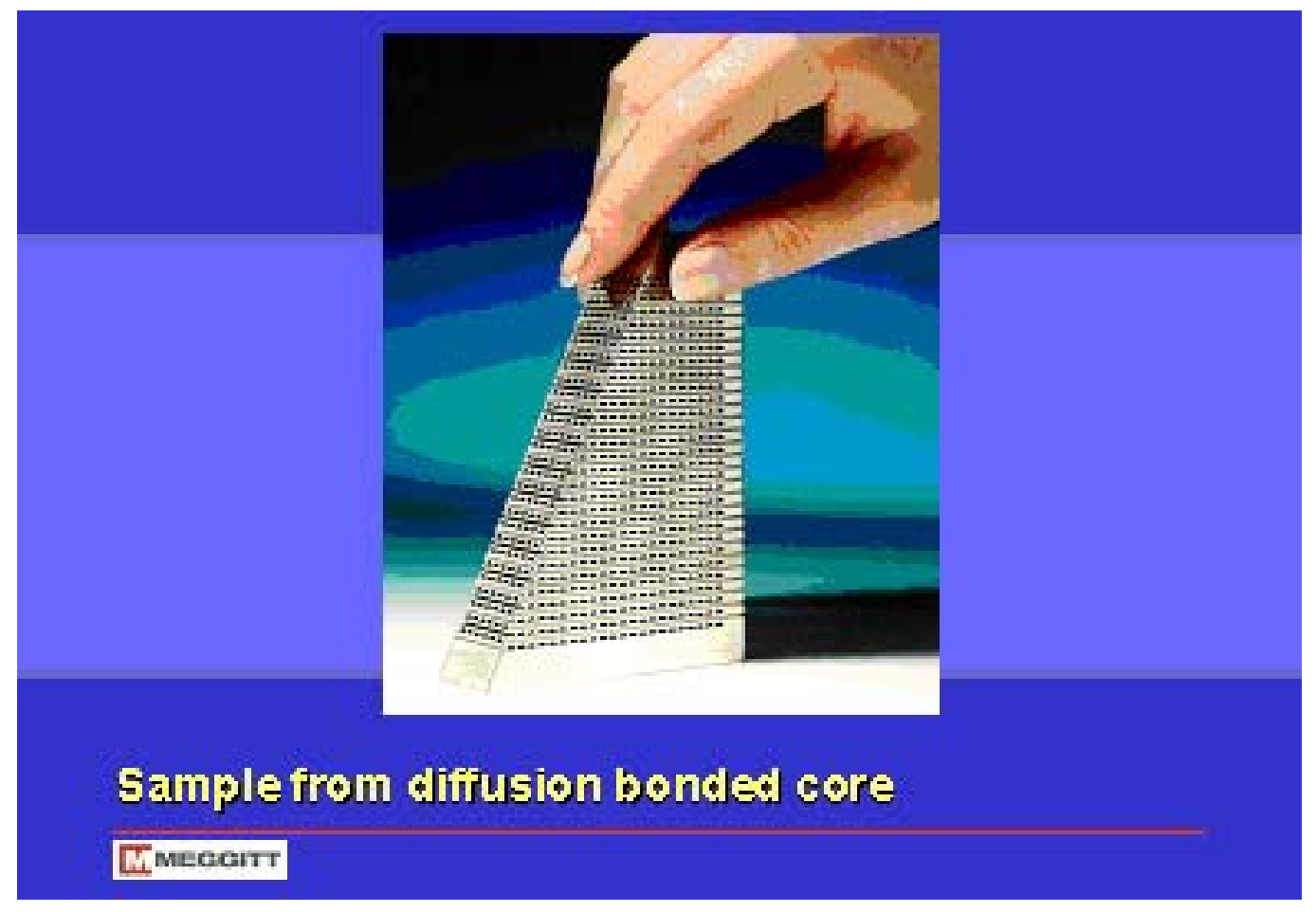

Figure 3.7 Example of PCHX core [7] 


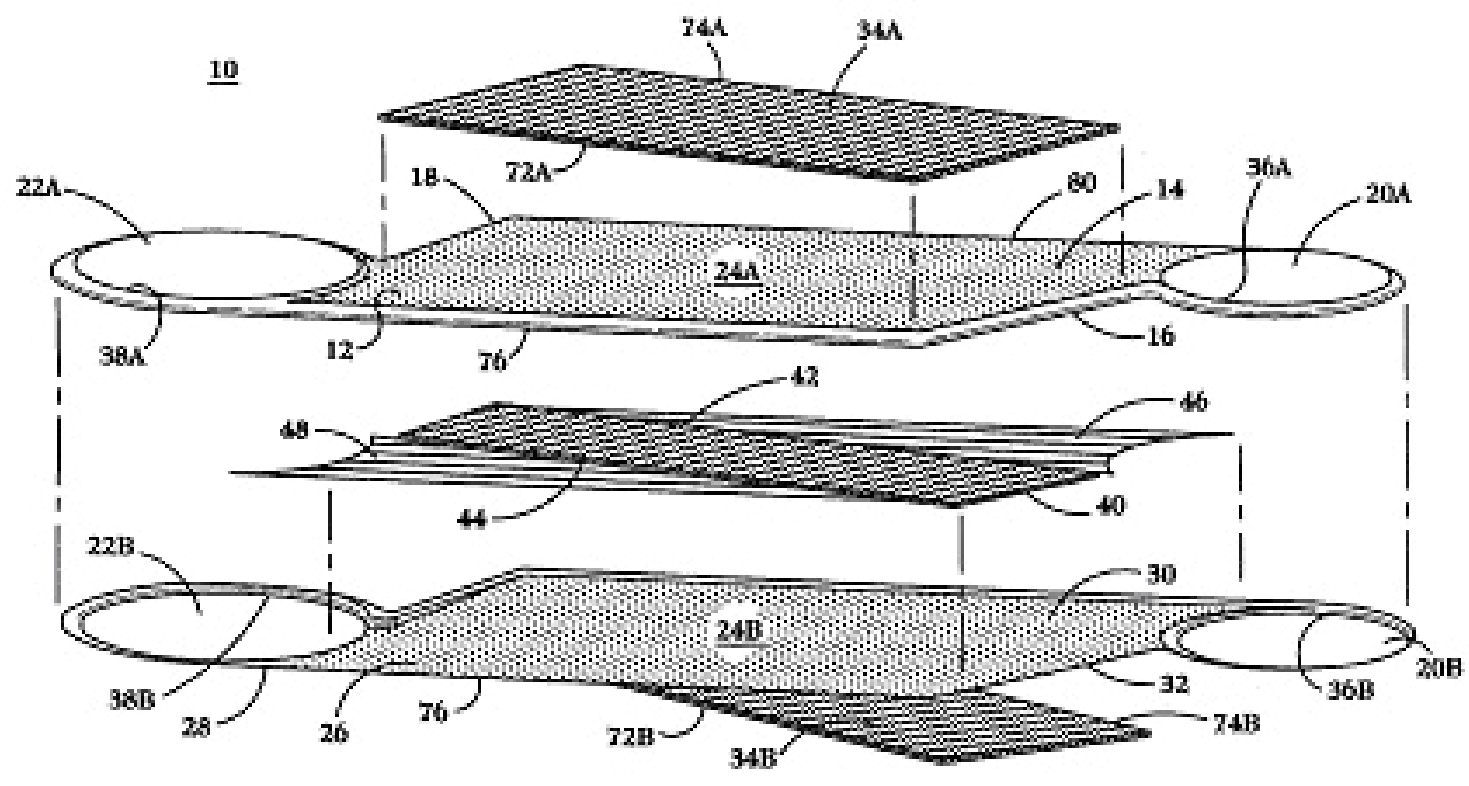

Figure 3.8 Unit-cell of plate fin heat exchanger [8]

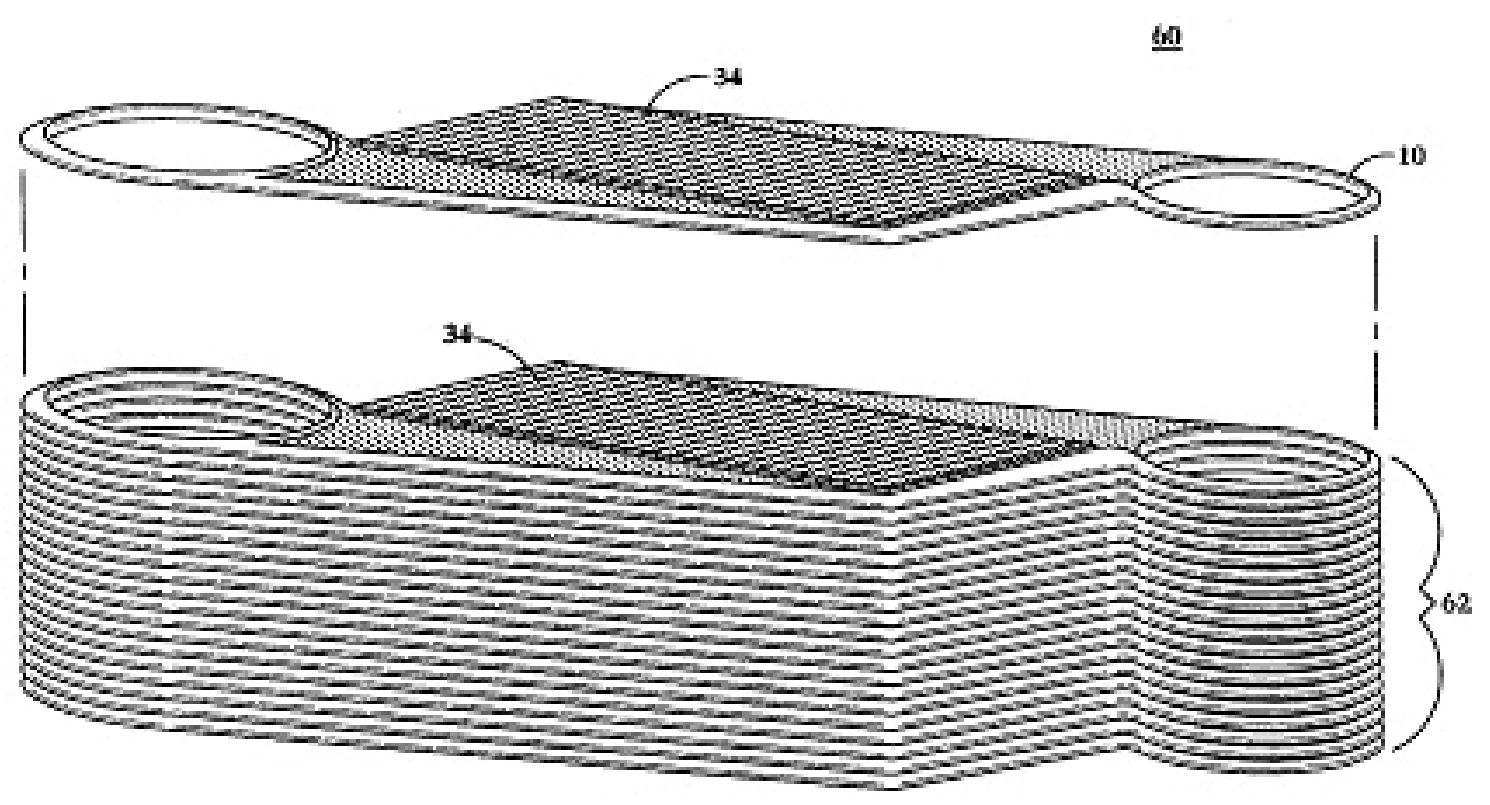

Figure 3.9 Stacking of plate fin heat exchanger unit-cells [8]

Using the semi-empirical heat exchanger model supplied by Concepts-NREC, parametric design calculations were conducted with design conditions slightly differing from that in Appendix A. The plate and fin geometry details for the selected configuration are as follows:

Cold-side Fin Layers $=1$

Hot-side Fin Layers $=2$ 
Plate Spacing $=1.65 \mathrm{~mm}(0.065 \mathrm{in})$

Parting Plate Thickness $=0.38 \mathrm{~mm}(0.015 \mathrm{in})$

Fin Material Thickness $=0.076 \mathrm{~mm}(0.003$ in)

Fin Spacing $=45$ fins/inch

Incoloy 800 was selected as the reference material to construct the IHX. Since the recuperator operates at a much lower temperature, it can use conventional and lower cost material such as 347 stainless steel. The relationships of friction and heat transfer with Reynolds number of the plate-fin surface 11.44-3/8W in Reference[9] were used.

A cautionary note is in order at this point. The number of materials that are qualified for use in this application are extremely limited even at $800^{\circ} \mathrm{C}$. At these temperatures the allowable stresses for ASME Section III application are of the order of only a few tens of an MPa. Thus, any design which uses an IHX must also include safety systems to assure that the differential pressure across the IHX remains extremely low at all times. As the temperature is increased to the $1000^{\circ} \mathrm{C}$ specified for the Next Generation Nuclear Plant (NGNP) the number of materials available for this application is effectively reduced to zero. Alloy 617, which has been proposed as a potential material and one for which some ASME code work has been done, will not be adequate at this temperature. Once again, the allowable stresses would be even lower than for $800^{\circ} \mathrm{C}$ operation.

An additional complication with the use of high temperatures in IHX applications is the requirement that creep and creep-fatigue be incorporated into the design rules. This has never been done in the nuclear industry. This inclusion will greatly complicate the code qualification process. In fact, from a materials qualification viewpoint, the qualification of a high temperature material will likely be a critical fence to the implementation of the gas cooled reactor technology.

The design conditions for the IHX and for the recuperator are given in Table 3.4 and Table 3.5, respectively. The results of the parametric design calculations for the IHX are given in Table 3.6 for the PFHX configuration. For the recuperator, the results are given in Table 3.7. The estimated costs are scaled from Appendix A based on the weight.

From Table 3.6, it can be seen that, as the effectiveness increases 5 percentage points, the heat exchanger volume is roughly doubled, thus the cost is also roughly double. We can see, from Table 3.7, low pressure losses result in larger heat exchanger volume and thus higher cost if the effectiveness is fixed. With the same effectiveness, the recuperator occupies a larger volume than the IHX since the former operates at a lower pressure. Combined with Appendix A, it can be noted that the PCHE design is large and costly, but provides less development risk in the present timeframe. The IRES PFHX design is promising in terms of the volume and cost.

Currently, the upper limit temperature of the Heatric heat exchanges in service is $800^{\circ} \mathrm{C}$. Withstanding a higher temperature up to $900^{\circ} \mathrm{C}$, high-temperature alloys such as Incoloy $800 \mathrm{pr}$ 617 would be required. The designers in Heatric and IRES expressed confidence that there are no technical barriers to use high-temperature alloys but that this would require a very significant development effort[10]. For the depressurization accident, in which the secondary loop depressurizes while the primary loop remains at normal pressure, the IHX will experience a 
pressure differential of 7.8MPa. The management of this kind of loss-of-pressure scenario needs further study. In the long term, for operation at very high temperatures an alternate technology will be required. These technologies include ceramic and composite materials.

Table 3.4 IHX design conditions

\begin{tabular}{|l|c|c|}
\hline & Primary (hot) side & Secondary (cold) side \\
\hline Working fluid & Helium & Helium \\
\hline Flow rate $(\mathrm{kg} / \mathrm{s})$ & 126.7 & 126.7 \\
\hline Inlet temperature $\left({ }^{\circ} \mathrm{C}\right)$ & 900 & 488.8 \\
\hline Outlet temperature $\left({ }^{\circ} \mathrm{C}\right)$ & 509 & 879.4 \\
\hline Inlet Pressure $(\mathrm{MPa})$ & 7.73 & 7.99 \\
\hline Requirement & $\begin{array}{l}\text { Effectiveness } \geq 90 \% \\
\text { Primary pressure loss } \leq 2 \% \\
\text { Secondary pressure loss } \leq 2 \%\end{array}$ \\
\hline
\end{tabular}

Table 3.5 Recuperator design conditions

\begin{tabular}{|l|c|c|}
\hline & Hot side & Cold side \\
\hline Working fluid & Helium & Helium \\
\hline Flow rate $(\mathrm{kg} / \mathrm{s})$ & 126.7 & 126.7 \\
\hline Inlet temperature $\left({ }^{\circ} \mathrm{C}\right)$ & 511 & 74 \\
\hline Outlet temperature $\left({ }^{\circ} \mathrm{C}\right)$ & 96 & 489 \\
\hline Inlet Pressure $(\mathrm{MPa})$ & 2.75 & 8.0 \\
\hline Requirement & $\begin{array}{l}\text { Effectiveness }=95 \% \\
\text { Hot side pressure loss } \leq 1.8 \% \\
\text { Cod side pressure loss } \leq 0.8 \%\end{array}$ \\
\hline
\end{tabular}

Table 3.6 IHX parametric design calculation results for PFHX configuration

\begin{tabular}{|l|c|c|c|}
\hline Effectiveness & $95 \%$ & $92.5 \%$ & $90 \%$ \\
\hline Hot side pressure loss, \% & 0.49 & 0.46 & 0.65 \\
\hline Cold side pressure loss, \% & 1.41 & 1.39 & 1.40 \\
\hline Number of modules & 18 & 18 & 18 \\
\hline Module width, $\mathrm{W}, \mathrm{mm}$ & 635 & 635 & 432 \\
\hline Module length, $\mathrm{L}, \mathrm{mm}$ & 676 & 475 & 411 \\
\hline Module height, H, mm & 1143 & 1003 & 1125 \\
\hline Total core volume, $\mathrm{m}^{\wedge}$ 3 & 11.2 & 7.49 & 5.15 \\
\hline Estimate total core weight $(\mathrm{kg})$ & 26,310 & 17,620 & 12,120 \\
\hline Approximate HX cost, US 2001 \$M & 3.1 & 2.1 & 1.4 \\
\hline
\end{tabular}

NOTES:

A "module" design as following: 


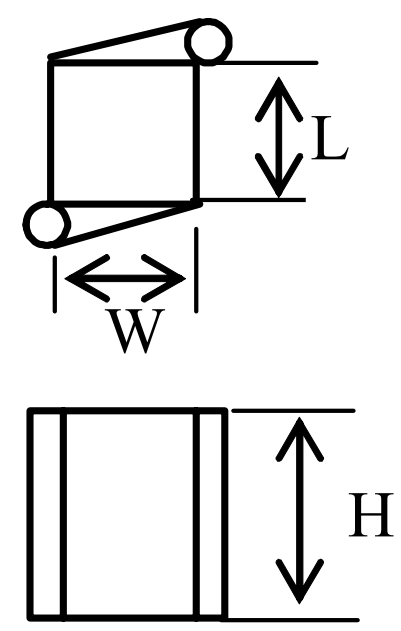

Table 3.7 Recuperator parametric design calculation results for PFHX configuration

\begin{tabular}{|l|c|c|c|}
\hline Effectiveness & $95 \%$ & $95 \%$ & $95 \%$ \\
\hline Hot side pressure loss, \% & 0.80 & 1.38 & 1.77 \\
\hline Cold side pressure loss, \% & 0.334 & 0.575 & 0.738 \\
\hline Number of modules & 18 & 18 & 18 \\
\hline Module width, $\mathrm{W}, \mathrm{mm}$ & 762 & 762 & 762 \\
\hline Module length, $\mathrm{L}, \mathrm{mm}$ & 561 & 660 & 711 \\
\hline Module height, $\mathrm{H}, \mathrm{mm}$ & 1455 & 1118 & 991 \\
\hline Total core volume, $\mathrm{m}^{\wedge} 3$ & 15.3 & 13.2 & 12.4 \\
\hline Estimate total core weight $(\mathrm{kg})$ & 35,960 & 31,220 & 29,320 \\
\hline Approximate HX cost, US 2001 \$M & 0.60 & 0.52 & 0.49 \\
\hline
\end{tabular}

- Helium gas turbomachinery design consideration

High pressure $(7 \sim 8 \mathrm{MPa})$ and low pressure ratio $(2.5 \sim 3)$ characterize the nuclear helium gas turbine cycle. For helium turbomachines, the Mach number limitation is no longer the limitation for the design due to the high sonic speed of helium. Thus, the blade tip circumferential stress becomes the design limitation in the determination of the number of stages.

There are two basic types of turbine - radial flow and axial flow. The axial turbine is normally more efficient than the radial type. The radial turbine has a relative higher efficiency only for handling low mass flow. In application, the vast majority of gas turbines are axial type. For the MPBR cycle, an axial turbine is suitable. In the MPBR, the highest turbine inlet temperature will be lower than $880^{\circ} \mathrm{C}$. Using single crystal super alloys avoids the necessity of blade cooling.

Since the pressure ratio for compressors is in the low range there are two options for the compressor configurations - centrifugal and axial. The predominant advantage of the centrifugal 
design is the ability to operate over a wider range of mass flow than an equivalent axial compressor, which allows for advantages in control system design. However, the axial compressor has the potential for higher efficiency and smaller size.

The aerodynamic design for a 5 stage centrifugal compressor, an $8+1$ stage axi-centrifugal compressor and an axial turbine has been conducted by Concepts-NREC. The design results are included in Appendix B.

Table 3.8 Design details of Recuperator with effectiveness of $95 \%$, pressure loss of $0.8 \%$ (hot side), $0.334 \%$ (cold side)

\begin{tabular}{|c|c|c|}
\hline & Hot side & Cold side \\
\hline Flow rate $(\mathrm{kg} / \mathrm{s})$ & 126.7 & 126.7 \\
\hline Inlet pressure (MPa) & 511 & 74 \\
\hline Inlet temperature $\left({ }^{\circ} \mathrm{C}\right)$ & 96 & 489 \\
\hline Outlet temperature $\left({ }^{\circ} \mathrm{C}\right)$ & 2.75 & 8.0 \\
\hline Core Height $(\mathrm{mm})$ & \multicolumn{2}{|l|}{1455} \\
\hline Core Width (mm) & \multicolumn{2}{|l|}{762} \\
\hline Core Length $(\mathrm{mm})$ & \multicolumn{2}{|l|}{561} \\
\hline Inlet Header Width & NA & $152 \mathrm{~mm}(6 \mathrm{in})$ \\
\hline Outlet Header Width & NA & $203 \mathrm{~mm}(8 \mathrm{in})$ \\
\hline Heat transfer area $\left(\mathrm{m}^{\wedge} 2\right)$ & 1749 & 842.5 \\
\hline Plate spacing & $3.3 \mathrm{~mm}(0.13 \mathrm{in})$ & $1.65 \mathrm{~mm}(0.065 \mathrm{in})$ \\
\hline Fin spacing (1/in) & 45 & 45 \\
\hline Fin thickness & $\begin{array}{l}0.076 \mathrm{~mm}\left(3 \times 10^{\wedge}-3\right. \\
\text { in) }\end{array}$ & $0.076 \mathrm{~mm}\left(3 \times 10^{\wedge}-3 \mathrm{in}\right)$ \\
\hline Free Flow/Face Area & 0.825 & 0.825 \\
\hline Surface/Volume $\left(\mathrm{m}^{\wedge} 2 / \mathrm{m}^{\wedge} 3\right)$ & 4734 & 4685 \\
\hline Hydraulic radius (m) & $1.743 \times 10^{\wedge}-4$ & $1.716 \times 10^{\wedge}-4$ \\
\hline Total heat transfer (MW) & \multicolumn{2}{|l|}{273} \\
\hline Reynolds number & 296 & 615 \\
\hline Heat transfer coefficient $\mathrm{W} / \mathrm{m}^{2} \mathrm{~K}$ & 2061.7 & 3200.1 \\
\hline Fin efficiency & 0.353 & 0.538 \\
\hline Surface efficiency & 0.424 & 0.64 \\
\hline Side pressure loss $(\%)$ & 0.80 & 0.334 \\
\hline Inlet header pressure loss (\%) & NA & 0.055 \\
\hline Outlet header pressure loss (\%) & NA & 0.081 \\
\hline No. of Passage pairs & 255 & \\
\hline Weight $(\mathrm{kg})$ & 35960 & \\
\hline
\end{tabular}

\subsection{Schematic Of Current Design}

The goal of the design is to provide a plant with high efficiency, low investment, modularity and less cost for maintenance. 
As described in the previous section, given the advances of technology associated with heat exchanger design, the fabrication of an IHX working under severe conditions such as high temperatures and high pressures has become a viable alternative as long as the upper temperature is kept within the $800^{\circ}$ range. This has allowed us to choose an indirect cycle design.

Helium gas is used as the working fluid in both the primary system and the secondary system. The flow schematic of the current MPBR design is shown in Figure 3.10. A three-shaft arrangement with three-stage intercooling is adopted in the power conversion unit. In the primary system, the hot helium goes into the hot side of the IHX, and after transferring heat to the power conversion unit, flows to the circulator. The circulator is located in the primary outlet of the IHX, taking advantage of the low temperature condition. It provides the pressure head to overcome the pressure losses caused by the resistance through the primary cycle. Thereafter, the helium goes back to the upper plenum of the reactor core to finish the loop. In the secondary system, the helium leaving the cold side of the IHX is expanded sequentially in the high-pressure (HP), low-pressure (LP) and power turbines. The turbine exhaust helium enters the low pressure side of the recuperator and transfers its heat to the high pressure side helium. Before the helium enters a compressor, it is cooled to $30^{\circ} \mathrm{C}$. The helium is compressed to $8.0 \mathrm{MPa}$ by four compressors and then enters the high pressure side of the recuperator. After heat is recovered, the helium flows into the cold side of the IHX. The helium, heated in the IHX, leaves the cold side of the IHX to start the next cycle once again. The helium pressure in the cold side of the IHX is $0.1 \mathrm{MPa}$ higher than that of the hot side to prevent radioactive product transfer to the power conversion unit. In order to provide a stream for cooling of the reactor pressure vessel (RPV), a separate vessel cooling heat exchanger is used. On the primary side, helium is bled from the outlet of the circulator and is cooled to a low temperature level. The cooled gas then enters the annulus cavity between the reactor core barrel and RPV to cool the RPV. On the secondary side of the vessel cooling heat exchanger, cold helium is diverted from the outlet of the high-pressure compressor. The precooler and intercoolers are helium/water heat exchangers. The cold water is provided by the cooling tower.

\subsection{Summary}

An indirect, recuperated and intercooled gas turbine cycle is chosen for the power conversion system to be coupled with the pebble bed reactor system. A three-shaft arrangement is selected for the shaft configuration. Two types of compact heat exchanger technology are provided to meet the MPBR cycle requirements. The design of helium gas turbomachinery has been investigated and the aerodynamic design has been conducted by Concepts-NREC. The cycle schematic is provided.

To define the optimum cycle parameters and to investigate the stability of the cycle, models need to be developed. Next section will describe the model developed. 


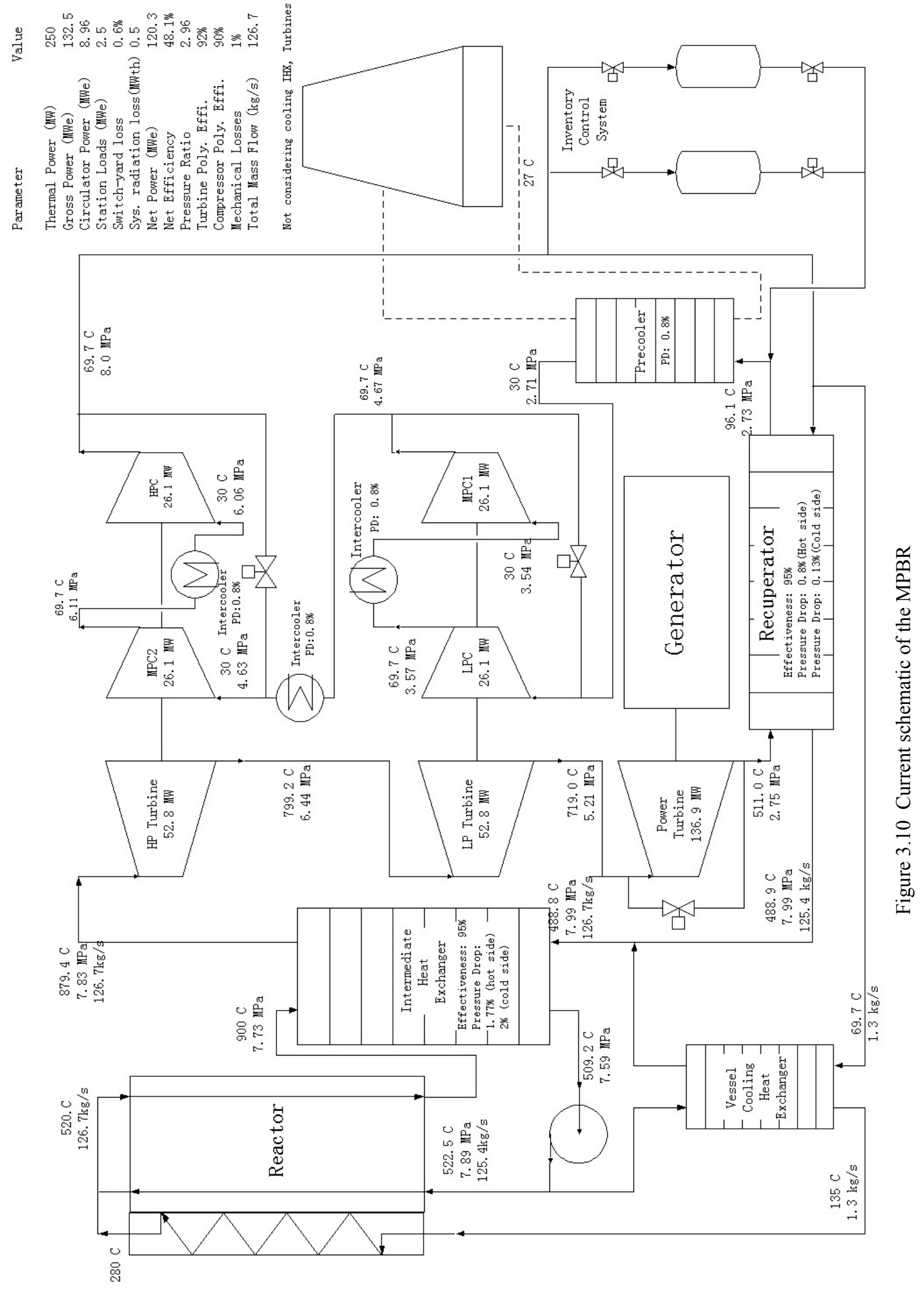




\section{References: Section 3}

[1] T. A. Galen, "Comparison between air and helium for use as working fluids in the energy conversion cycle of the MPBR", MIT MS thesis, May, 2000.

[2] H. Cohen, G. F. C. Rogers, H. I. H. Saravanamuttoo, "Gas turbine theory", third edition, John Wiley \& Sons, 1987.

[3] Reiner Decher, "Energy conversion systems, flow physics and engineering", Oxford university press, 1994.

[4] V. Dostal, M. Driscoll, P. Hejzlar, N.E. Todreas, “A supercritical $\mathrm{CO}_{2}$ gas turbine power cycle for next-generation nuclear reactors", Proceedings of ICONE $10^{\text {th }}$ international conference on nuclear engineering, Arlington, Virginia, April 14-18, 2002.

[5] ESKOM, "Pre-qualification request - the pebble bed modular reactor", 1996.

[6] Ronald C. Pampreen, "Compressor surge and stall”, Concepts ETI, Inc, Norwich, Vermont, USA, 1993.

[7] "PCHE printed circuit heat exchangers", promotional literature from Heatric, 46 Holton Road, Poole, Dorset BH 16 6LT, England.

[8] Malcolm S. Child, James B. Kesseli, "Unit construction plate fin heat exchanger", U.S. Patent Number 5,983,992, Nov. 16, 1999.

[9] W. M. Kays, A. L. London, “Compact heat exchangers”, third edition, McGraw-Hill book company, 1984.

[10] Personal email communication with Mr. Eli Demetri of Concepts-NREC, Dec. 12, 2000. 


\section{Results: Model development}

\subsection{Introduction}

As shown in Figure 3.10, the MPBR power plant utilizes a pebble bed reactor as heat source and indirectly couples a Brayton cycle for electricity production. The nominal cycle efficiency and pressure ratio can be calculated based on the performances of the components in the cycle. With the balance of plant, any variation of one parameter affects the plant performance. For example, replacing the recuperator with an effectiveness of $95 \%$ with another at $90 \%$ one will change the cycle efficiency and the optimum pressure ratio. To define the plant nominal parameters and to conduct parametric analysis, developing a steady state model is required. This model must be flexible enough to deal with cycle variations, such as intercooling stage number and other changes.

Because of the high heat capacity of the core, the time constant of the core is relatively large while the response of the turbomachines to load transients is fast. In order to investigate the interaction of the dynamic behavior of the reactor system and power conversion system, a dynamic model had to be developed for simulation of the overall nuclear gas turbine plant, including the pebble bed reactor, IHX and power conversion system. Similar work has been done with other gas turbine nuclear power plant systems [1,2,3,4,5]. The dynamic model was used to investigate the interaction of the components design and dynamics, to explore the control strategy and to determine the control structure such as the bypass valve position and inventory control system. Also, component design will be evaluated and load transient simulation will be performed. The model consists of several sub-models corresponding to the plant components, such as the reactor, heat exchangers, gas turbines, gas compressors and bypass valves. It performs the control scheme using a PI (proportional-plus-integral) controller algorithm. Using the dynamic model, the operability of the power conversion system design have been evaluated.

After providing the plant flow schematic and the losses of the components, the plant nominal parameters can be chosen by using the steady state model. The dynamic model gives the plant transient performance based on the physical parameters of the components. The following describes the steady state and dynamic models.

\subsection{Steady State Model Development}

\subsubsection{Component Losses}

A real cycle consists of a series of components and the connecting piping. The components include a heat source device, turbo-machines and heat exchangers as well as valves. Energy losses occur as the working fluid passes through the components. Due to the component losses, the real cycle efficiency will be lower than that of an ideal cycle. For turbomachines, helium compression and expansion are irreversible adiabatic processes and thus result in an entropy increase. In the heat exchanger, the cold side outlet temperature will be lower than that of the hot inlet because the heat exchanger volume is limited due to 
economic and transport reasons. The working fluid through the cycle will experience pressure losses due to friction. In the following sections, the different kinds of component losses will be described. We will then describe the efficiency of turbomachinery, the heat exchanger effectiveness, pressure loss, shaft mechanical loss, motor efficiency, and the energy loss caused by the frequency converter and gear box. Before introducing the compressor and turbine efficiencies, we need to be familiar with the concept of variation of specific heat and fluid stagnation properties.

\section{- Variation of Specific Heat}

The properties, $C p$ and $\gamma$, for helium remain constant over the temperature range of interest. However, for other real gases such as air and $\mathrm{CO}_{2}, \mathrm{Cp}$ is a function of temperature alone over normal working ranges of temperature and pressure. The variations of $C p$ and $\gamma$ have an effect on estimating the cycle performance and it is necessary to take into account the variations in values, which are caused by the change in conditions through the cycle.

\section{- Stagnation Properties}

Since fluid velocities are very high in the turbomachinery, the kinetic energy change between the inlet and outlet cannot be ignored. If a gas stream of enthalpy $h$ and velocity $C$ comes to rest adiabatically and without work transfer, its enthalpy rises to $h_{0}$ called the stagnation (or total) enthalpy. The energy equation then becomes:

$$
\left(h_{0}-h\right)+\frac{1}{2}\left(0-C^{2}\right)=0
$$

thus $h_{0}$ is defined by

$h_{0}=h+\frac{C^{2}}{2}$

The stagnation enthalpy includes the kinetic energy. When the fluid is a perfect gas, $h=$ $C p T$. Thus, we define the stagnation temperature $T_{0}$ by:

$T_{0}=T+\frac{C^{2}}{2 C p}$

$T$ is referred to as the static temperature, and $\frac{C^{2}}{2 C p}$ is called the dynamic temperature. For example, if a $800^{\circ} \mathrm{C}$ helium stream flows in the piping with velocity of $120 \mathrm{~m} / \mathrm{s}$, the stagnation temperature is about $801.4^{\circ} \mathrm{C}$.

It can be seen from the energy equation that $T_{0}$ will remain constant if there is no heat or work transfer. For an adiabatic compression process, the work input to the compressor is

$W_{C}=\left(C p_{2} T_{2}+\frac{1}{2} C_{2}^{2}\right)-\left(C p_{1} T_{2}+\frac{1}{2} C_{1}^{2}\right)=C p_{2} T_{20}-C p_{1} T_{10}$

where subscript 1 refers to inlet and 2 refers to outlet. For any real gas, it is sufficiently accurate to take the mean $\bar{C} p$ to calculate compressor input work [6]:

$W_{C}=\bar{C} p\left(T_{20}-T_{10}\right)$ 
The stagnation (or total) pressure is defined in a similar way as the stagnation temperature but with one more restriction: that the gas comes to rest not only adiabatically but also reversibly, i.e. isentropically. Thus the stagnation pressure $p_{0}$ is defined as:

$$
\frac{p_{0}}{p}=\left(\frac{T_{0}}{T}\right)^{\gamma /(\gamma-1)}
$$

Substituting eqn. (4.2) in eqn. (4.5) and using $C p=R \frac{\gamma}{\gamma-1}$ and $p R=\rho R T$, one gets

$$
p_{0}=p\left(1+\frac{\rho C^{2}}{2} \times \frac{\gamma-1}{\gamma}\right)^{\gamma / \gamma-1}
$$

By taking the first two terms of the binomial expansion, this becomes

$p_{0}=p+\frac{\rho C^{2}}{2}$

where $p$ is referred to as the static pressure, $\rho$ is the fluid density and $C$ is fluid velocity. When the Mach number is low and compressibility effects are negligible, equation (4.6) approaches equation (4.7). Since the sonic velocity of helium is high, for simplicity, for steady state calculations, equation (4.7) is used here.

For showing the relation of static and stagnation properties virtually, a T-s diagram is shown in Figure 4.2 to depict a compression process from static states 1 to 2 . If an isentropic compression process takes place to reach the same actual outlet stagnation pressure, the ideal state would be $2^{\prime}$.

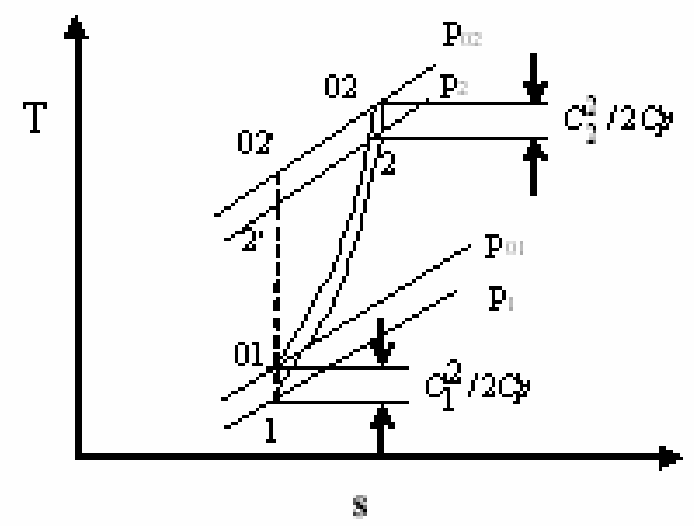

Figure 4.1 Stagnation and static states in compression process

\section{- Compressor and Turbine Efficiencies}

The efficiencies of turbomachines usually compare the actual work with ideal work transfer. For turbomachines, the process is essentially adiabatic, thus the ideal process is isentropic, 
and the efficiency is the isentropic efficiency. For the compressor, the process is shown in Figure 4.1:

$\eta_{\text {SC }}=\frac{\Delta h^{\prime}}{\Delta h}=\frac{C p\left(T_{02}^{\prime}-T_{01}\right)}{C p\left(T_{02}-T_{01}\right)}$

For the turbine, it is the inverse ratio

$\eta_{s t}=\frac{T_{02}-T_{01}}{T_{02}^{\prime}-T_{01}}$

where subscript 1 and 2 refer to the component inlet and outlet, respectively. From (4.8), we can change it to:

$T_{02}-T_{01}=\frac{T_{01}}{\eta_{S C}}\left(\frac{T_{02}^{\prime}}{T_{01}}-1\right)$

For an isentropic process with a perfect gas, the relation of temperature $T$ and pressure $p$ is:

$\operatorname{Tp}^{\frac{1-\gamma}{\gamma}}=$ constant

where $\gamma=C p / C v$; and with equation (4.5), for the compressor, we can derive:

$T_{02}-T_{01}=\frac{T_{01}}{\eta_{S C}}\left[\left(\frac{p_{02}}{p_{01}}\right)^{(\gamma-1) / \gamma}-1\right]$

By a similar method, for the turbine:

$T_{01}-T_{02}=\eta_{s t} T_{01}\left[1-\left(\frac{1}{\left(p_{01} / p_{02}\right)}\right)^{(\gamma-1) / \gamma}\right]$

However, there is a disadvantage with using isentropic efficiency in the analysis of a Brayton power cycle or in quality comparisons of turbomachinary aerodynamic design: the isentropic efficiency is a function of pressure ratio. The following example illustrates this point.

Suppose that there are two identical compressors with the same isentropic efficiency and same enthalpy rise, and we put them together as a new compressor to obtain higher pressure ratio (Figure 4.2)[7]. Consider the enthalpy rise: the actual enthalpy rise of the new compressor is the sum of that of the individual ones; but the isentropic enthalpy rise of the new compressor will be less than the sum of that of individual ones.

For each original compressor $\Delta h_{01}=\Delta h_{02}$

$\eta_{s, \text { old }}=\frac{\Delta h_{01 s}}{\Delta h_{01}}=\frac{\Delta h_{02 s}}{\Delta h_{02}}$

thus 


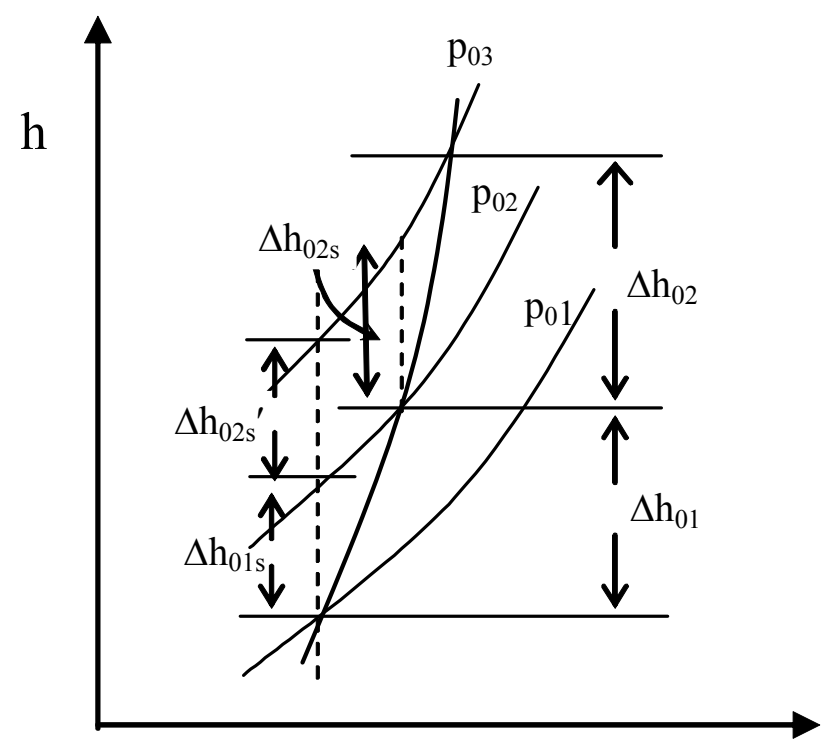

S

Figure 4.2 Isentropic efficiency of a combined compressor [7]

$\Delta h_{01 s}=\Delta h_{02 s}$

But, because the vertical distance between two constant pressure lines in the h-s diagram increases when the entropy increases, thus

$\Delta h_{2 s}>\Delta h_{2 s}^{\prime}$

For the new compressor

$\eta_{s, \text { new }}=\frac{\Delta h_{01 s}+\Delta h_{02 s^{\prime}}}{\Delta h_{01}+\Delta h_{02}}<\frac{\Delta h_{01 s}+\Delta h_{02 s}}{\Delta h_{01}+\Delta h_{02}}$

Therefore

$\eta_{s, \text { new }}<\eta_{s, \text { old }}$

That means the isentropic efficiency of the new compressor is less than that of the old compressors. In other hands, the aerodynamic performance of the new compressor appears to be poorer than that of the original units.

This leads to the concept of polytropic efficiency, $\eta_{\mathrm{p}}$, which is defined as the isentropic efficiency of a process as the pressure ratio approaches unity. According to the definition, the polytropic efficiency can be derived from the isentropic efficiency. For a detailed derivation, see reference [7]. 
With perfect gas, for the compressor:

$\left.\frac{T_{02}}{T_{01}}=\left(\frac{p_{02}}{p_{01}}\right)^{\left(\frac{R}{C p} \frac{1}{\eta_{p c}}\right.}\right)$

and for the turbine

$\frac{T_{02}}{T_{01}}=\left(\frac{p_{02}}{p_{01}}\right)^{\left(\frac{R}{C p} \eta_{p t}\right)}$

again, subscripts 1 and 2 refer to inlet and outlet of the component, $T_{0}$ and $p_{0}$ are the stagnation temperature and pressure, respectively, $R$ is the gas constant, $C p$ is specific heat.

In the cycle design process, the optimum cycle pressure ratio would vary if the cycle configuration is changed or component performance varies. Thus we adopt polytropic efficiency for turbomachines in the steady state heat balance model.

\section{- Heat Exchanger Effectiveness}

Heat exchanger effectiveness is usually used to describe heat exchanger performance. It is defined by the ratio of the actual to the maximum possible rate of heat transfer.

$\varepsilon_{h x}=\frac{q}{q_{\max }}=\frac{(\dot{m} C p)_{h}\left(T_{h, \text { in }}-T_{h, \text { out }}\right)}{(\dot{m} C p)_{\min }\left(T_{h, \text { in }}-T_{C, \text { in }}\right)}$

where $(\dot{m} C p)_{\text {min }}$ is defined as the smaller of $(\dot{m} C p)_{h}$ and $(\dot{m} C p)_{c}$, the subscripts $h$ and $c$ refer to the hot and cold fluids, respectively. $\dot{m}$ is the fluid mass flowrate and $C p$ is the specific heat. As the effectiveness of a heat exchanger is specified, knowing the mass flowrate in each side, the hot side inlet temperature and the cold side inlet temperature can determine the other two temperatures: hot side outlet, cold side outlet. In general, the higher effectiveness the larger the volume is for the same configuration.

\section{- Pressure Loss}

Working fluid friction and flow cross-section area changes result in pressure losses in the heat exchangers, reactor core, inlet and outlet of each component, and piping connecting components. In the overall cycle performance evaluation, losses in piping are usually included in the associated component losses. The pressure loss is expressed as a percentage pressure loss:

$$
P D=\frac{p_{\text {in }}-p_{\text {out }}}{p_{\text {in }}} \times 100 \%
$$

where $p_{\text {in }}$ and $p_{\text {out }}$ are inlet and outlet pressure for a component, respectively. The pressure losses of heat exchangers are obtained based on design results. The pressure loss through the 
pebble bed reactor core can be calculated using the following empirical relation provided from KFA based on the operational experience in the AVR and THTR:

$\Delta p=\psi \cdot \frac{1-\varepsilon}{\varepsilon} \cdot \frac{H}{d_{p}} \cdot \frac{1}{2 \rho} \cdot\left(\frac{\dot{m}}{A}\right)^{2}$

where $\psi=\frac{320}{\frac{\operatorname{Re}}{1-\varepsilon}}+\frac{6}{\left(\frac{\operatorname{Re}}{1-\varepsilon}\right)^{0.1}}$

$\operatorname{Re}=\frac{\dot{m} \cdot d_{p}}{A \cdot \mu}$

$\rho$ is the density of helium $\left(\mathrm{kg} / \mathrm{m}^{3}\right), R e$ is Reynolds number, $\varepsilon$ is pebble bed void fraction, $H$ is core height $(\mathrm{m}), d_{p}$ is pebble diameter $(\mathrm{m}), A$ is cross-section area of the core $\left(\mathrm{m}^{2}\right), \dot{m}$ is the helium mass flowrate $(\mathrm{kg} / \mathrm{s})$, and $\mu$ is helium dynamic viscosity $(\mathrm{kg} / \mathrm{m} \mathrm{s})$.

This equation applies to the following range:

$1 \leq \frac{\mathrm{Re}}{1-\varepsilon} \leq 10^{5}$

$0.36 \leq \varepsilon \leq 0.42$

For the MPBR, in the current stage we use the PBMR core geometry; it yields a core pressure loss of $\triangle P=76 \mathrm{kPa}$ using the parameters listed in Table 4.1. The percentage of core pressure loss is about $1 \%$.

Table 4.1 MPBR reactor core pressure loss calculation

\begin{tabular}{|l|l|l|l|}
\hline $\mathrm{H}=9.04 \mathrm{~m}$ & $\mathrm{~A}=9.62 \mathrm{~m}^{2}$ & $\mathrm{~d}_{\mathrm{p}}=0.06 \mathrm{~m}$ & $\varepsilon=0.39$ \\
\hline $\mathrm{T}_{\text {core, } \text { in }}=520^{\circ} \mathrm{C}$ & $\mathrm{T}_{\text {core }, \text { out }}=900^{\circ} \mathrm{C}$ & $\mathrm{p}_{\text {core, in }}=7.89 \mathrm{MPa}$ & $\dot{m}=126.7 \mathrm{~kg} / \mathrm{s}$ \\
\hline$\rho=3.83 \mathrm{~kg} / \mathrm{m}^{3}$ & $\mu=4.496 \times 10^{-5} \mathrm{~kg} / \mathrm{m} \mathrm{s}$ & $\mathrm{Re}=1.757 \times 10^{4}$ & $\Delta p=76 \mathrm{kPa}$ \\
\hline
\end{tabular}

\section{- Mechanical Loss}

For all our cycle designs, the compressors are driven directly by the turbine without any intermediate gearing. Therefore, the loss that occurs is due to only the bearing friction and windage. This value is very small and usually assumed $1 \%$ of the turbine power [6]. If we denote the transmitted efficiency as $\eta_{\mathrm{m}}$, the turbine power needed for driving the compressor:

$W_{t}=\frac{1}{\eta_{m}} W_{C}$

where $W_{t}$ is the turbine power and $W c$ is the power consumed by the compressor.

\section{- Motor Efficiency}


In indirect cycle design, an $\mathrm{AC}$ motor drives the circulator in the primary system. Energy loss in the motor occurs when electrical energy is transferred into mechanical energy. This efficiency is referred to as motor efficiency, for which $98 \%$ is adopted here.

\section{- Frequency Converter, Gear Box}

If a single-shaft arrangement is adopted in the PCU, the generator and all the turbomachines are mounted on a single shaft. Within the stress limitation, increasing the rotational speed of turbines and compressors results in an increase in their efficiency and reduction in their dimensions. This requires the introduction of a frequency converter between the generator and the grid or a speed reducing gearbox between the power turbine and the generator in a single-shaft arrangement. It enables the selection of the optimum rotational speed for turbomachines, independent of the synchronized frequency of the grid. However, the maximum practical power output for which a gearbox is used is around 80MW[8]. A typical gearbox has a design point efficiency of $97.5 \% \sim 99 \%$ [8]. Similar to the gearbox, a frequency converter also causes energy losses; in the MPBR power range, it will reduce the efficiency of the generator by around $2 \%[9]$.

\section{- Other Losses}

In practice, a quantity of compressed gas is always bled off for cooling turbine discs and blade roots. The amount of bleed gas is about 1 2 per cent of the total mass flowrate[6]. And since the turbine inlet temperature is high, it is necessary to cool the turbine casing in order to reduce its thickness. Also, because the IHX pressure vessel is an ASME Section III boundary, it requires cooling to make the temperature of the pressure boundary lower than $427^{\circ} \mathrm{C}$. Since the helium molecular weight is lower compared with other gases, it is prone to leak, therefore, leakage will be an issue in compressors. The cooling stream and leakage gas consume extra work and thus reduce the cycle efficiency.

\subsubsection{Steady State Cycle Calculation Procedure}

Before assessing the effect of the component loss on the cycle general performance, we outline the method of calculating the cycle net efficiency in any particular case for specified values of the design parameters. These parameters include the core inlet/outlet temperatures, reactor thermal power, the polytropic efficiencies of turbomachines, heat exchanger effectiveness, and pressure losses.

In the calculation, we assume each compressor has same pressure ratio. Determination of the cycle efficiency for an indirect cycle proceeds the following known parameters: The cycle maximum pressure, i.e. high-pressure compressor outlet pressure; precooler and intercooler gas side outlet temperatures; the pressure drop between the IHX cold side outlet and the hot side inlet. The procedure for calculation is as follows:

(1) Assume an overall pressure ratio of the secondary cycle (PCU);

(2) From the reactor thermal power and reactor inlet/outlet temperatures, determine the primary cycle mass flowrate; 
(3) From the HP compressor outlet pressure and pressure losses, get the IHX cold side outlet pressure;

(4) From the IHX cold side outlet pressure and the IHX pressure drop, obtain the IHX hot side inlet pressure, and then, get the IHX hot side outside pressure and circulator inlet pressure;

(5) From the IHX hot side inlet pressure, obtain the core inlet pressure and circulator outlet pressure.

(6) For the circulator, knowing the outlet temperature, outlet pressure, inlet pressure and mass flowrate, calculate the inlet temperature by equation (4.10), and then the input work. If $C p$ varies with temperature, a method of successive approximation is required. First, guess a value of $C p$, calculate the inlet temperature, take the more accurate mean value of $C p$ and re-calculate the inlet temperature;

(7) From the IHX hot inlet temperature and outlet temperature, using the definition of heat exchanger effectiveness, get the IHX cold side inlet temperature $T_{I H X, c, \text { in }}$ and outlet temperature;

(8) Set the gas mass flowrate of the secondary cycle (PCU) the same as that of the primary cycle;

(9) From the overall pressure ratio and HP compressor outlet pressure, obtain the LP compressor inlet pressure and pressure ratio. The pressure ratio for each compressor is obtained by

$$
P R_{C}=\left[\frac{P R}{\left(1-P D_{I C \# 1}\right)\left(1-P D_{I C \# 2}\right)\left(1-P D_{I C \# 3}\right)}\right]^{1 / 4}
$$

where $P R$ is the total pressure ratio, $P D_{I C \# 1,} P D_{I C \# 2,} P D_{I C \# 3}$ are the intercoolers' percentage pressure losses;

(10) Knowing the LP compressor inlet pressure, inlet temperature and pressure ratio, obtain the outlet temperature by using equation (4.12) and input work;

(11) Use the same method to get the other compressors' outlet temperature and input work;

(12) HP turbine output work is equal to the sum of work of the MP compressor \#2 and HP compressor and mechanical losses. For the HP turbine, knowing output work, inlet temperature and mass flowrate, calculate the outlet temperature. Then, from the temperature ratio and inlet pressure, obtain the outlet pressure by using equation (4.13);

(13) Same method for the LP turbine;

(14) From LP compressor inlet pressure, precooler and recuperator hot side pressure losses, get the power turbine outlet pressure;

(15) For the power turbine, knowing inlet pressure, outlet pressure and inlet temperature, calculate the outlet pressure, temperature and output work;

(16) For the recuperator, from the hot side inlet temperature, and cold side inlet temperature, calculate the cold side outlet temperature $T_{R c p, c, o}$;

(17) If the recuperator cold side outlet temperature differs from the IHX cold side inlet temperature, go back to step (1) to assume a new overall pressure ratio. 
For finding quickly the proposed overall pressure ratio, the following numerical method is used: Take a pressure ratio obviously much less than the proposed overall pressure ratio as $P R_{1}$, and take another pressure ratio obviously much larger than the proposed overall pressure ratio as $P R_{2}$, i.e. for helium cycle, 1.001 and 25.0 for $P R_{1}$ and $P R_{2}$, respectively. Then use the mean pressure ratio $P R$ to calculate $T_{R c p, c, o}$. If $T_{I H X, c, \text { in }}$ is less than $T_{R c p, c . o}$, set $P R$ as $P R_{1}$, otherwise, set $P R$ as $P R_{2}$. After that, re-calculate the mean pressure ratio $P R$ to get a new $T_{R c p, c, o \text {. }}$ The iteration continues until it satisfies the converge criterion:

$$
\frac{\left|T_{I H X, c, i n}-T_{R c p, c, o}\right|}{T_{I H X, c, i n}}<1 \times 10^{-6}
$$

The flowchart of the calculation is shown in Figure 4.3. The plant net efficiency is defined as the percentage of the electrical power output from the plant to the reactor thermal power:

$\eta_{\text {net }}=\frac{P_{\text {net }}}{Q} \times 100 \%$

where $\mathrm{P}_{\text {net }}$ is the electrical power output from the plant to the customer and $\mathrm{Q}$ is the reactor thermal power.

In the cycle parametric analysis, for reasons of simplification, here define a cycle efficiency as follows:

$\eta_{\text {cycle }}=\frac{W_{p t} \cdot \eta_{m} \cdot \eta_{\text {gen }}-W_{\text {cir }} / \eta_{\text {motor }}-W_{s l}}{Q} \times 100 \%$

where $W_{p t}$ is the power turbine output work, $W_{\text {cir }}$ is the circulator input work, $W_{s l}$ is station load, $Q$ is the reactor thermal power, $\eta_{m}$ is the mechanical transmitted efficiency, $\eta_{g e n}$ is the generator efficiency and $\eta_{\text {motor }}$ is the efficiency of the motor for driving the circulator. The system radiation loss, switch-yard loss, working fluid leakage losses and component cooling losses are not taken into account in the above definition. 


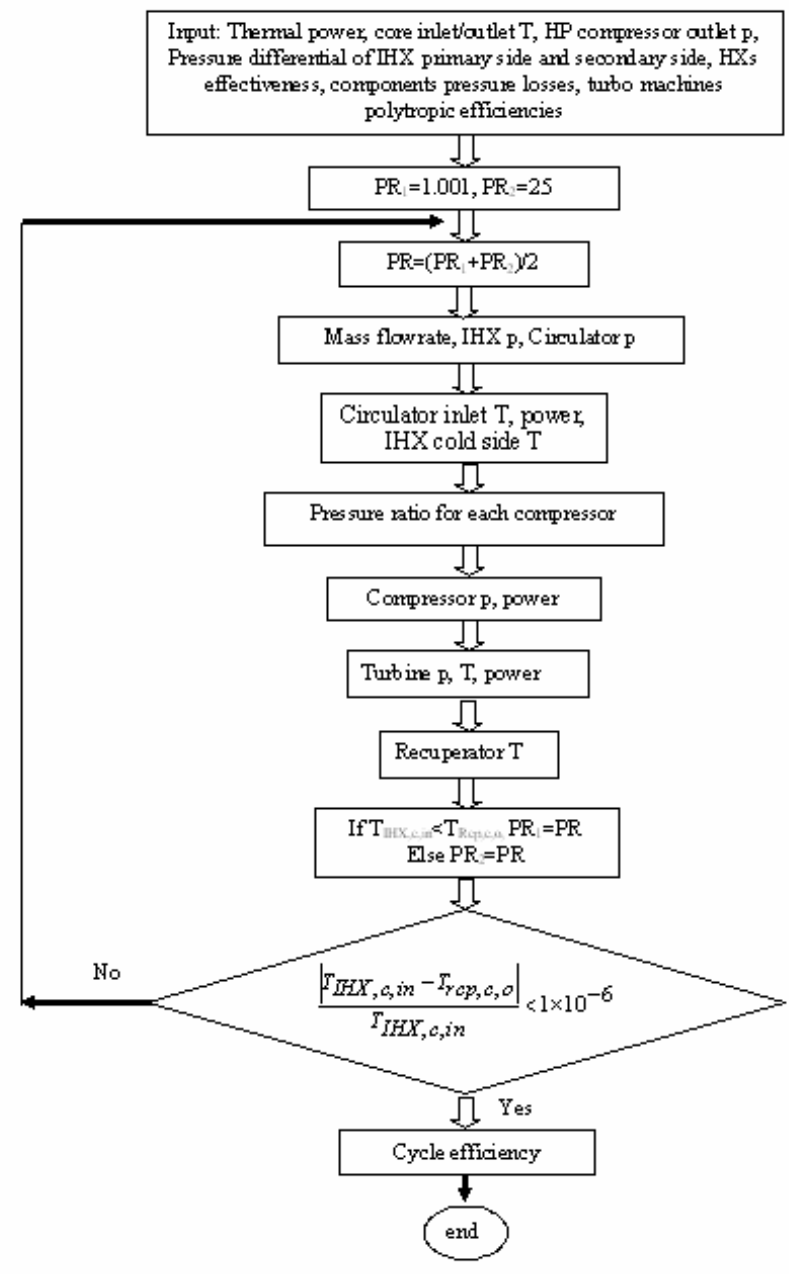

Figure 4.3 Flowchart of the steady state calculation

\subsubsection{Estimation Of Cooling Mass Flowrate For Reactor Pressure Vessel And IHX Vessel}

In the MPBR, the material for the reactor pressure vessel (RPV) was chosen as A508/A533. The upper temperature limitation of this material is $375^{\circ} \mathrm{C}$. During normal operation, the RPV temperature is required to be $280^{\circ} \mathrm{C}$. The average temperature of the core barrel is estimated to be $390^{\circ} \mathrm{C}$ by PBR_SIM(Pebble Bed Reactor_SIMulation )[10]. To satisfy the RPV temperature limitation, a cooling helium stream is provided in the cavity between the core barrel and the reactor vessel, as shown in Figure 3.10. Figure 4.4 shows the diagram of the cavity between the core barrel and the RPV. In this section we estimate the mass flowrate of the cooling helium stream. 
As we know the core barrel temperature $T_{\mathrm{cb}}$, the RPV temperature $\mathrm{T}_{\mathrm{RPV}}$, the core barrel radius $R_{c b}$, the $R P V$ radius $R_{R P V}$, and the height $H$, the heat transferred from the core barrel to the RPV through conduction and radiation can be estimated [11]:

$h_{\text {gap }}=\frac{k_{H e}}{\delta}+\frac{\sigma}{\frac{1}{\varepsilon_{c b}}+\frac{1}{\varepsilon_{R P V}}-1} \frac{T_{c b}^{4}-T_{R P V}^{4}}{T_{c b}-T_{R P V}}$

where $h_{\text {gap }}$ is the heat transfer coefficient for the cavity between the core barrel and the pressure vessel; $\mathrm{T}_{\mathrm{cb}}$ is the core barrel temperature; $\mathrm{T}_{\mathrm{RPV}}$ is the pressure vessel temperature;

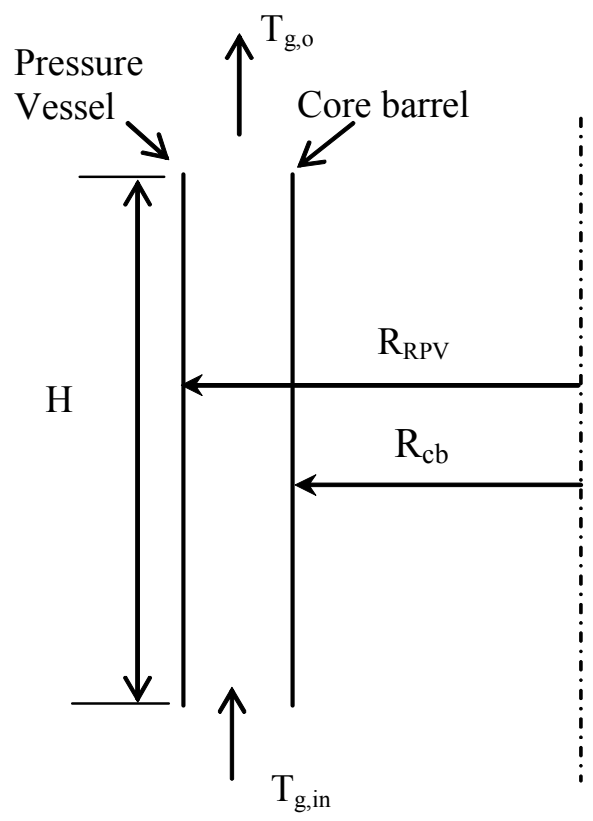

Figure 4.4 Reactor pressure vessel cooling

$\mathrm{k}_{\mathrm{He}}$ is the thermal conductivity of helium; $\delta$ is the cavity width; $\sigma$ is the Stefan-Boltzman constant; $\varepsilon_{\mathrm{cb}}, \varepsilon_{\mathrm{RPV}}$ are the surface emissivities of the core barrel and pressure vessel, respectively. The heat transferred from the core barrel to the pressure vessel by conduction and radiation can be estimated:

$Q_{c b-R P V}=2 \pi R_{c b} \cdot H \cdot h_{g a p} \cdot\left(T_{c b}-T_{R P V}\right)$

where $\mathrm{H}$ is the pressure vessel height.

The heat removed by the cooling stream in the cavity from the core barrel and the pressure vessel can also be estimated. With regard to the gas flow in the cavity, it is considered as laminar when the Reynolds number $\operatorname{Re}<2300$ and turbulent when $\operatorname{Re}>10^{4}$. In the range of 
$2300<\operatorname{Re}<10^{4}$, the flow region is the transition flow. Approximate correlations for calculating the friction factor and heat transfer coefficient are used [12]:

$$
f=A+\frac{B}{\operatorname{Re}^{1 / m}}
$$

For $\mathrm{Re}<2100, \mathrm{~A}=0, \mathrm{~B}=16$, and $\mathrm{m}=1$; for $2100<\mathrm{Re}<4000, \mathrm{~A}=0.0054, \mathrm{~B}=2.3 \times 10^{-8}$, and $\mathrm{m}=-$ $2 / 3$; for $\operatorname{Re}>4000, A=1.28 \times 10^{-3}, B=0.1143$, and $m=3.2154$.

In the range of $2100<\operatorname{Re}<10^{6}$, and $0<\operatorname{Pr}<\infty$, the heat transfer coefficient can be estimated[12]:

$N u^{10}=N u_{l}^{10}+\left(\frac{\exp [(2200-\mathrm{Re}) / 365]}{N u_{l}^{2}}+\frac{1}{N u_{t}^{2}}\right)^{-5}$

$N u_{l}=3.675$ for the uniform wall temperature boundary condition

$N u_{t}=N u_{0}+\frac{0.079(f / 2)^{1 / 2} \operatorname{Re} \operatorname{Pr}}{\left(1+\operatorname{Pr}^{4 / 5}\right)^{5 / 6}}$

$N u_{0}=4.8$ for the uniform wall temperature boundary condition where Pr is the Prandtl number.

The Dittus-Boelter equation is the most universally used correlation for turbulent flow as the fluid is heated [11]:

$$
N u=0.023 \operatorname{Re}^{0.8} \operatorname{Pr}^{0.4}
$$

for $0.7<\operatorname{Pr}<100, \operatorname{Re}>10,000$, and $\mathrm{L} / \mathrm{D}>60$; $\mathrm{L}$ is heated length and $\mathrm{D}$ is hydraulic diameter.

The relationship between the Nusselt number and the heat transfer coefficient is:

$h=\frac{k_{H e} \cdot N u}{D_{H}}$

where $\mathrm{h}$ is heat transfer coefficient; $\mathrm{k}_{\mathrm{He}}$ is the helium conductivity, $\mathrm{D}_{\mathrm{H}}$ is the heated diameter. After obtaining the heat transfer coefficient, the heat removed from the pressure vessel and core barrel by the cooling stream can be calculated with a similar method to equation (4.23).

Considering the heat balance about the pressure vessel:

$Q_{c b-R P V}=Q_{R P V, c}+Q_{r a d}$ 
where $\mathrm{QRPV}_{\mathrm{RP}}$ is the total heat removed from the pressure vessel by the cooling helium; $\mathrm{Q}_{\mathrm{rad}}$ is the radiation heat loss from the pressure vessel to the environment, which is about $0.5 \mathrm{MW}$ [10].

With respect to the cooling helium, the heat balance is:

$Q_{H e}=Q_{R P V, c}+Q_{c b, c}$

Where $\mathrm{Q}_{\mathrm{cb}, \mathrm{c}}$ is the heat removed from the core barrel; $\mathrm{Q}_{\mathrm{He}}$ is the total heat extracted by the cooling helium:

$Q_{H e}=\dot{m}_{H e} C p\left(T_{g, o u t}-T_{g, \text { in }}\right)$

where $\dot{m}_{H e}$ is the cooling helium mass flowrate, $T_{\mathrm{g}, \mathrm{in}}$ and $\mathrm{T}_{\mathrm{g} \text {, out }}$ are the inlet and outlet temperature, respectively, and $\mathrm{Cp}$ is the helium specific heat.

In this estimation, the radius of the pressure vessel is taken as $4.41 \mathrm{~m}$; the core barrel radius is $4.31 \mathrm{~m}$; the surface emissivities of the core barrel and pressure vessel are both taken as 0.6 . The pressure vessel height is $10 \mathrm{~m}$. With the assumption that the core barrel temperature is $390^{\circ} \mathrm{C}$, Figure 4.5 shows the amount of the cooling helium required to ensure that the RPV temperature is no more than $280^{\circ} \mathrm{C}$. We can see that the higher the cooling helium inlet temperature, the more helium coolant is required. Figure 4.6 shows that the variation of the core barrel temperature affects the heat transferred from the core barrel to the RPV by conduction and radiation and the required mass flowrate of the cooling helium.

As the core barrel temperature is $390^{\circ} \mathrm{C}$, about $1.3 \mathrm{~kg} / \mathrm{s}$ cooling helium is required for cooling the RPV to maintain its temperature no more than $280^{\circ} \mathrm{C}$.

The IHX consists of 18 identical modules: each of 6 IHX vessels to encapsulate 3 IHX modules as shown in Appendix C. The IHX vessel is approximately $6 \mathrm{~m}$ height and $2.3 \mathrm{~m}$ diameter. As described in Section 3, the IHX vessel is the ASME code section III boundary: the material temperature limitation is $427^{\circ} \mathrm{C}$. In the current IHX module design, the hot outlet helium is ducted to the chamber formed by the inner shell and the outer configuration of 3 modules. The inner shell temperature is the same as the IHX hot outlet temperature, $510^{\circ} \mathrm{C}$. Around the inner shell, a layer of insulation is used to decrease the temperature to $150^{\circ} \mathrm{C}$. The outer vessel is the pressure boundary whose temperature remains $150^{\circ} \mathrm{C}$. Within the annulus between the insulation layer and the pressure boundary, there is helium gas. Figure 4.7 shows the insulation approach. In the normal condition, the helium gas is stagnant. If the insulation layer breaks and causes a temperature increase with the pressure boundary, cold helium is bled from the HP compressor to cool the pressure boundary. 


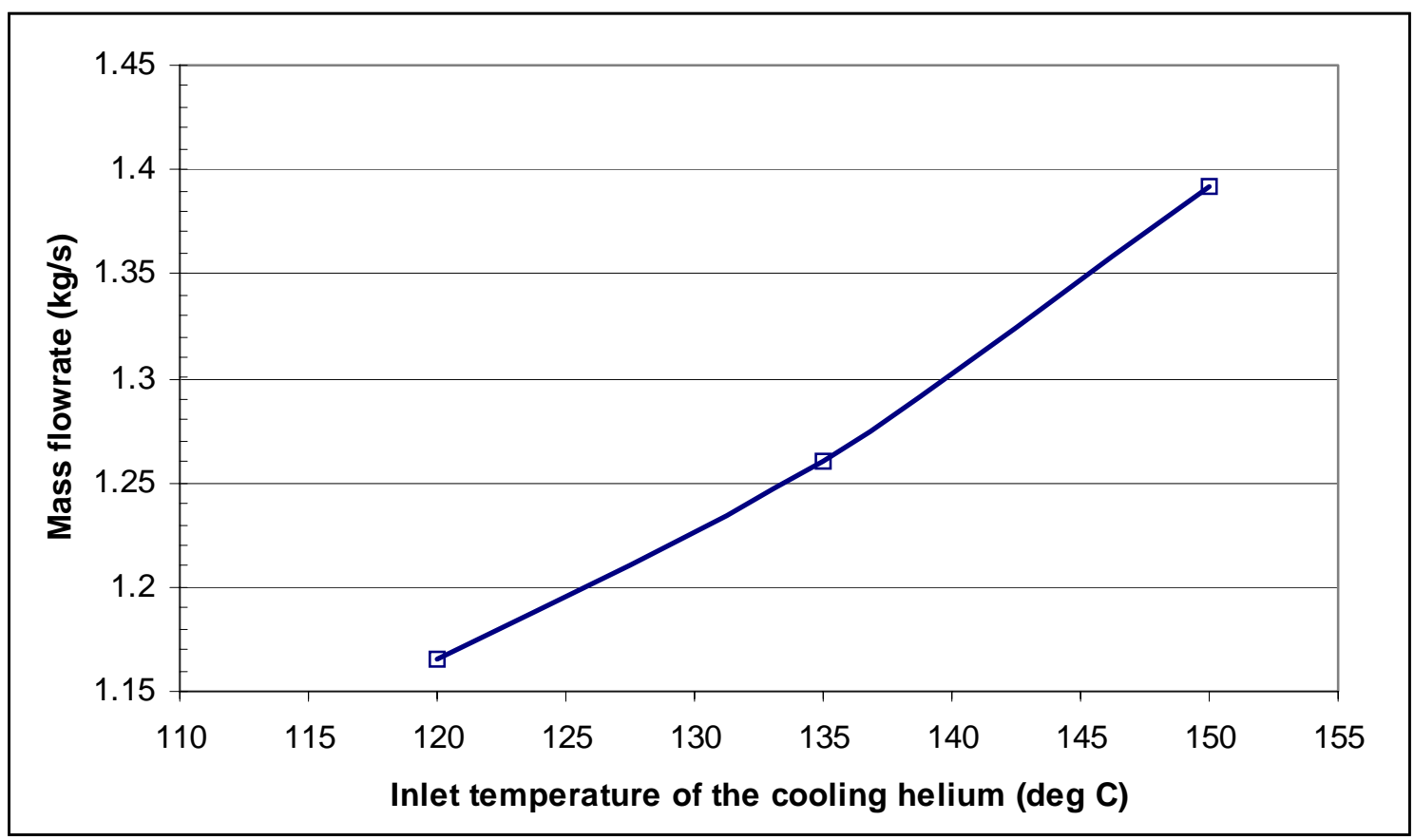

Figure 4.5 Cooling helium mass flowrate as a function of its inlet temperature at the condition that the core barrel and RPV temperatures are $390^{\circ} \mathrm{C}$ and $280^{\circ} \mathrm{C}$, respectively

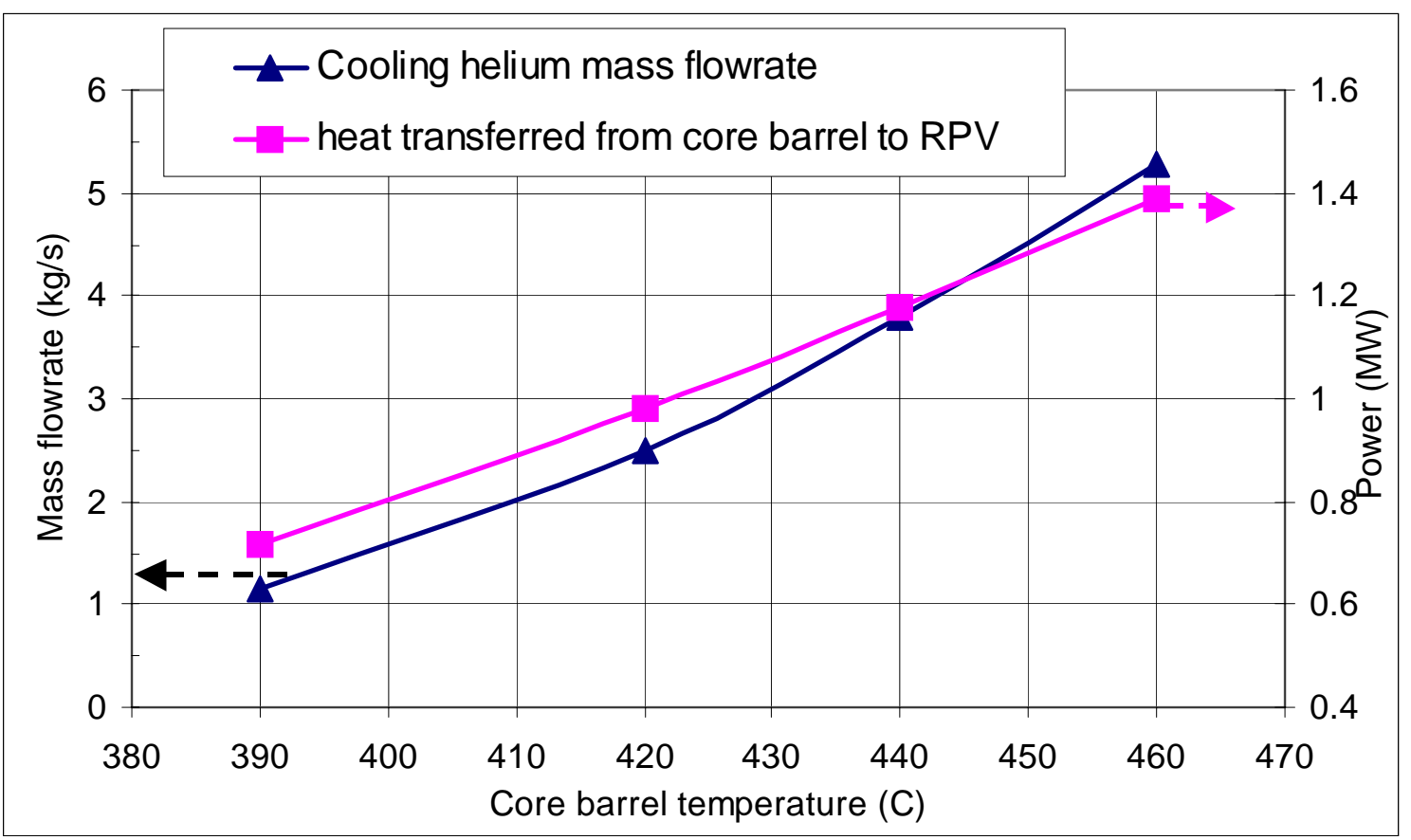

Figure 4.6 Core barrel temperature effects on the cooling helium mass flowrate and the heat transferred from core barrel to RPV for the condition of fixing the RPV temperature at $280^{\circ} \mathrm{C}$, and cooling helium temperature at $135^{\circ} \mathrm{C}$

The heat that radiates to the environment from the IHX outer vessel can be estimated: 


$$
Q=A \cdot \varepsilon \cdot C_{0} \cdot\left[\left(\frac{T_{1}}{100}\right)^{4}-\left(\frac{T_{2}}{100}\right)^{4}\right]
$$

$A$ is the outer vessel area; $\varepsilon$ is the outer vessel emissivity; $C_{0}$ is a constant, $5.67 \mathrm{~J} /\left(\mathrm{m}^{2} \mathrm{~s} \mathrm{~K} \mathrm{~K}^{4}\right)$; $\mathrm{T}_{1}$ is the outer vessel temperature; and $\mathrm{T}_{2}$ is the environment temperature.

We take the outer vessel diameter as $2.5 \mathrm{~m}$, the outer vessel emissivity as 0.6 , the environment temperature as $20^{\circ} \mathrm{C}$, and ignore the top and bottom cover. The radiation heat loss is about $3.95 \times 10^{4} \mathrm{~W}$ for each IHX vessel.

The insulation thickness can be estimated using the following equation[11]:

$T_{i s}-T_{i n s}=q^{\prime} \cdot \frac{1}{2 \pi k} \ln \left(\frac{R_{i n s}}{R_{i s}}\right)$

$\mathrm{T}_{\text {is }}$ is the inner shell temperature; $\mathrm{T}_{\text {ins }}$ is the outer side temperature of the insulation layer; $\mathrm{q}^{\prime}$ is linear power, $\mathrm{k}$ is the insulation conductivity; $\mathrm{R}_{\mathrm{ins}}$ is the insulation layer radius and $\mathrm{R}_{\mathrm{is}}$ is the inner shell radius. Taking the insulation conductivity as $0.2 \mathrm{~W} /\left(\mathrm{m}{ }^{\circ} \mathrm{C}\right)$, the insulation thickness is about $90 \mathrm{~mm}$ to ensure a temperature decrease from $510^{\circ} \mathrm{C}$ to $150{ }^{\circ} \mathrm{C}$.

Three IHX modules

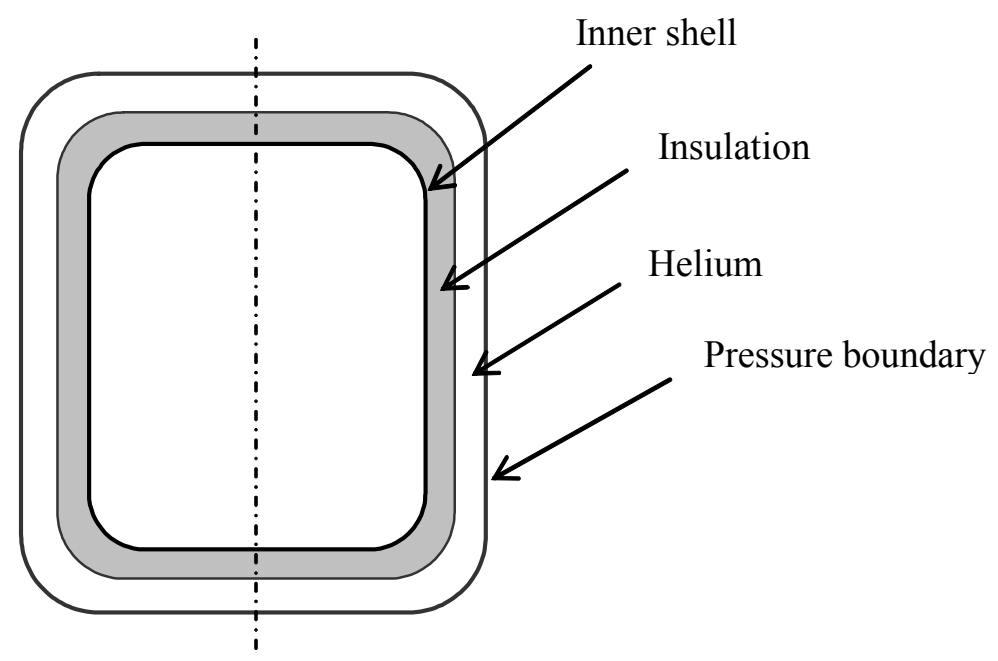

Figure 4.7 IHX vessel insulation

\subsection{Dynamic Model Development}

\subsubsection{Solution Approach}


For the reactor core and heat exchangers, their mathematical models are formed by a set of ordinary differential equations individually, while the performance of turbomachinery is represented by their characteristic maps. Obtaining the plant response to a transient requires that we simultaneously solve the ordinary differential equations of all the components and the characteristics of the turbomachines. For a closed cycle, the working fluid is re-circuited through the cycle. This configuration leads to a unique constraint for solving the ordinary differential equations and using the characteristics of the turbomachines - the calculate start and end points are physically the same point. As a result, the parameters, such as temperature, mass flowrate and pressure, of the start point should be equal to that of the end point within allowable error tolerance at a given time, as shown in Figure 4.8.

For calculation, we assume the parameters of arbitrary point \#1 (denoted as 1) in the closed cycle, at time $t$, are as follows: temperature $T_{1}^{t}$, pressure $p_{1}^{t}$ and mass flowrate $\dot{m}_{1}^{t}$. From point 1 , we then calculate the other points in sequence such as 2,3 , etc. When finishing the circuit, the end point (denoted as $1^{\prime}$ ) and the start point 1 are physically the same point.

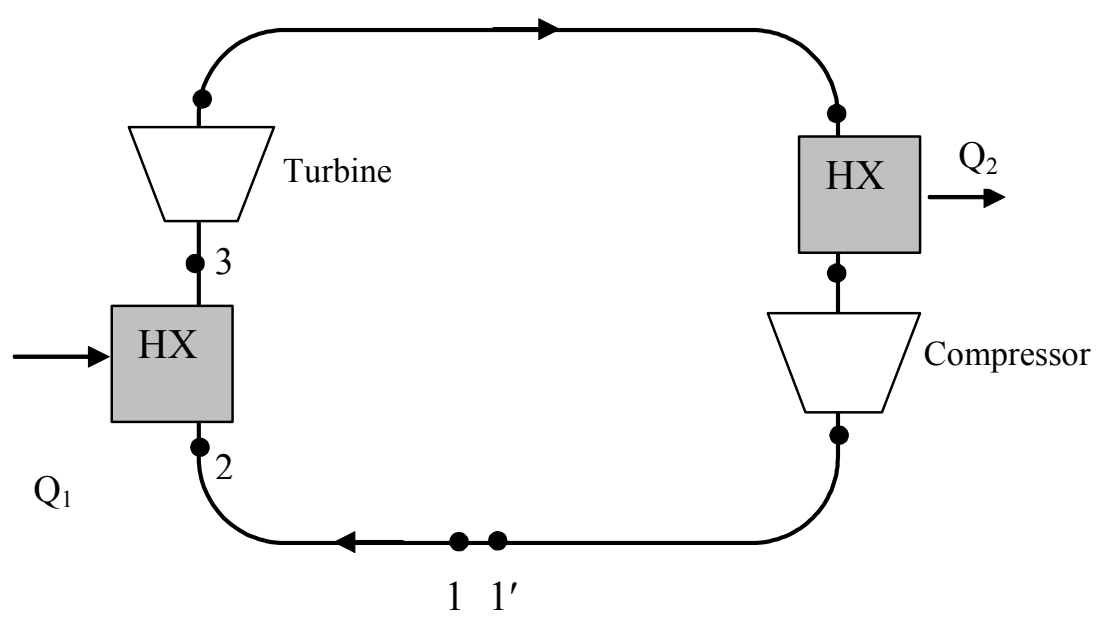

Figure 4.8 Calculation diagram for a closed cycle

The calculation convergence criteria are described as follows:

$$
\begin{aligned}
\frac{T_{1}^{t}-T_{1^{\prime}}^{t}}{T_{1}^{t}}<\varepsilon \\
\frac{p_{1}^{t}-p_{1^{\prime}}^{t}}{p_{1}^{t}}<\varepsilon
\end{aligned}
$$


$\frac{\dot{m}_{1}^{t}-\dot{m}_{1^{\prime}}^{t}}{\dot{m}_{1}^{t}}<\varepsilon$

where $\varepsilon$ is the allowable relative error. In the calculation for each time step, an iteration process is required. Taking pressure as an example, with a guessed $p_{1}$, we calculate $p_{1}^{\prime}$ and its error through the circuit. Then, we guess a new value for $p_{1}$. The iteration process continues until the convergence criterion is met. As the convergence criteria are satisfied, the calculations for next time step $\mathrm{t}+\Delta \mathrm{t}$ are performed.

After assessing the three parameters, pressure was found to be the most sensitive parameter for the power conversion system. Therefore, in this study, only a pressure iteration is performed. This results in some accuracy loss but simplifies iteration complexity.

The model is programmed by using the Advanced Continuous Simulation Language(ACSL)[13]. The pressure iteration is implemented by using an internal operator IMPLC, which is specifically designed for dealing with the algebraic loop. The solution process turns out to be solving differential algebraic equations(DAEs). The DAEs can be written as follows:

$$
\begin{aligned}
& \frac{d Y}{d t}=F(Y, Z) \\
& 0=G(Y, Z)
\end{aligned}
$$

Here Y's are the state variables and Z's are the algebraic variables. Since the residual relations for $\mathrm{Z}$ must be able to be solved, thus the partial of $\mathrm{G}$ with respect to $\mathrm{Z}$ must be nonsingular; i.e.,

$$
\left|\frac{\partial G(Y, Z)}{\partial Z}\right| \neq 0
$$

The method for solving (4.34) can be found in reference[14].

For the power conversion cycle, the algebraic constraint is

$0=p_{1}-p_{1}^{\prime}$

\subsubsection{Sub-Models Of Components}

\subsubsection{Reactor Model}

As the dynamic model simulates the interaction of the primary system and power conversion system, the reactor modeling can be simplified in comparison with the stand-alone reactor model involving a detailed core geometry analysis. The reactor model needs to deal with the core neutronics and heat transfer occurring within the reactor. Core neutronics takes into account the gross fission power variation due to the reactivity change. The reactor reactivity 
change comes from the following sources: control rod movement, fuel temperature change and fission product poisoning as well as the external reactivity disturbances. The heat transfer in the core is implemented by heat convection, conduction and radiation. In the dynamic model, the reactor model consists of a point kinetics model, a reactivity model and a two-dimensional thermal hydraulic model.

\section{- Integration Of The Core Model}

The calculation of internal heat generation within the reactor core is based on the point kinetics equations. The point kinetics model treats the core as a single point where the shape of neutron flux is time-independent. For simulation of the overall power plant, the point kinetics model is quite sufficient, and it is widely used in power plant simulators[1,2,3,5]. The thermal-hydraulic model accounts for calculating heat transfer within the pebble bed and reflector based on the total fission power and the power distribution; it gives the temperatures of fuel, reflector and coolant. Thus the temperature distribution in the core is obtained. Then, the reactivity caused by the fuel temperature change can be calculated. And the fission product poisoning can be obtained based on the fission power level. The reactivity is then fed back to the point kinetics model. The relationship of these models is schematically shown in Figure 4.9. The shape of the power distribution within the core, which is obtained from the stand-alone three-dimensional calculation, is assumed to remain fixed at any power condition; this assumption is also consistent with using the point kinetics equations.

\section{- Thermal Hydraulic Model}

In the primary system, after being cooled in the IHX, the helium is ducted back to the lower part of the reactor. Then it goes up through the channels in the side reflector and mixes in the top plenum. Thereafter, it passes through the pebble bed and is heated there. The hot helium then mixes in the bottom plenum and flows into the hot side of the IHX. In the IHX, the heat is transferred to the power conversion system.

The heat transfer from pebbles to the fluid takes place by forced convection. Heat transfer among pebbles is conducted by heat conduction and radiation. Heat conduction occurs inside the reflector and between the pebbles and reflector. In the radial direction, the heat is transferred from the core to the reactor vessel by means of conduction and radiation within the pebbles, conduction of the reflector and radiation through the helium channels. Also, heat convection occurs between the helium gas and the reflector when the helium goes up through the channels in the side reflector. The thermal energy in the outer surface of the core vessel is transmitted to the concrete containment by means of radiation through air and air natural convection. 


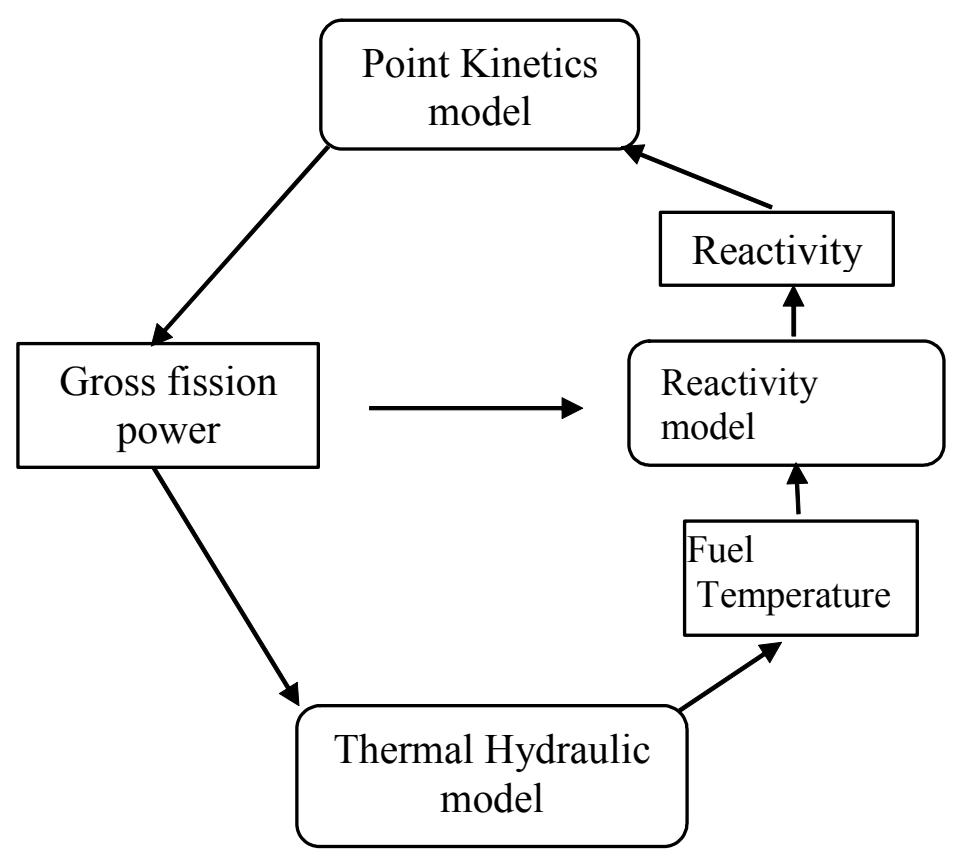

Figure 4.9 Interaction between the sub-models of the reactor model

During full power plant operation, heat loss from the reactor vessel is relatively small compared with the full thermal power. In the dynamic model, the simulation is for the overall power plant behavior. To simplify the calculation, the heat loss from the reactor vessel is assumed to be negligible, and the core barrel, helium channel and reactor vessel are excluded. This is quite sufficient for simulating the overall power plant. Currently, the MPBR adopts the ESKOM PBMR design. The core region consists of two regions: a passive region and an active region. The passive region contains the passive (pure) graphite pebble balls while the outer active core region is filled with fuel pebble balls. The outer diameters of the passive region and the active region are $1.75 \mathrm{~m}$ and $3.5 \mathrm{~m}$, respectively. The height of the active core is $8.5 \mathrm{~m}$. The thickness is assumed to be $1.15 \mathrm{~m}$ for all reflectors. In order to model the heat transfer phenomena above mentioned, the two-dimensional thermal hydraulic model nodal scheme shown in Figure 4.10 is used. In the axial direction, there are 12 axial layers: 10 core nodes, 1 top reflector and 1 bottom reflector. In the radial direction, there are 8 radial divisions: 1 inner graphite pebble, 5 fuel pebble divisions and 2 side reflector nodes. The temperature is uniform within a node in the numerical treatment. The coolant is assumed to be well-distributed in the pebble bed region and fluid flow in the radial direction is assumed negligible. The coolant mass flowrate fractions of the nodes in the radial direction of the pebble bed region, from inner to outer, are $0.22,0.156,0.156$, $0.156,0.156,0.156$, respectively.

The following energy conservation equation written in cylindrical coordinates is used to determine the temperature distribution as follows: 


$$
\begin{aligned}
\rho(T) C_{p}(T) \frac{\partial T(r, z, t)}{\partial t}= & \frac{1}{r} \frac{\partial}{\partial r}\left(k(r, z, t) r \frac{\partial T(r, z, t)}{\partial r}\right)+\frac{\partial}{\partial z}\left(k(r, z, t) \frac{\partial T(r, z, t)}{\partial z}\right)+ \\
& q_{f}(r, z, t)+q_{C}(r, z, t)+q_{R}(r, z, t)
\end{aligned}
$$

where $\mathrm{T}$ is temperature $(\mathrm{K}), \rho$ density $\left(\mathrm{kg} / \mathrm{m}^{3}\right), \mathrm{C}_{\mathrm{P}}$ specific heat $(\mathrm{J} / \mathrm{kg} . \mathrm{K})$, $\mathrm{k}$ thermal conductivity $(\mathrm{W} / \mathrm{m} . \mathrm{K}), \mathrm{q}_{\mathrm{f}}$ fission power $\operatorname{density}\left(\mathrm{W} / \mathrm{m}^{3}\right), \mathrm{q}_{\mathrm{C}}$ convective heat transfer $\operatorname{density}\left(\mathrm{W} / \mathrm{m}^{3}\right)$, and $\mathrm{q}_{\mathrm{R}}$ radiation heat transfer $\operatorname{density}\left(\mathrm{W} / \mathrm{m}^{3}\right)$.

It is assumed that all the solid materials in one node have a uniform temperature and fission energy generation density. Equation (4.37) can be expressed in discrete form with the aid of Figure 4.11, which shows an enlarged node on the $j_{\text {th }}$ radial division and the axial $i_{\text {th }}$ division. For node $(i, j)$, the finite difference equation can be derived by first principles and integrated along the radial direction from $R_{j-1}$ to $R_{j+1}$ as follows:

$\rho C_{p} \frac{d T_{i, j}}{d t} \pi\left(R_{j+1}^{2}-R_{j}^{2}\right) L=\sum Q_{n o d e, i j}+Q_{f i s s i o n, i j}-Q_{C, i j}+Q_{R, i j}$

$\sum Q_{\text {node }, i j}$ is the total heat transfer from the node $(i, j)$ to its adjacent nodes:

$$
\begin{aligned}
& \sum Q_{\text {node }, i j}=\frac{2 \pi R_{j} L}{\Delta r_{i j, j-1}} \bar{k}_{i j, j-1}\left(T_{i, j-1}-T_{i, j}\right)+\frac{2 \pi R_{j+1} L}{\Delta r_{i j, j+1}} \bar{k}_{i j, j+1}\left(T_{i, j+1}-T_{i, j}\right) \\
& +\frac{\pi\left(R^{2} i, j+1-R^{2} i, j\right)}{L} \bar{k}_{i j, i-1}\left(T_{i-1, j}-T_{i, j}\right)+\frac{\pi\left(R^{2} i, j+1-R^{2} i, j\right)}{L} \bar{k}_{i j, i+1}\left(T_{i+1, j}-T_{i, j}\right)
\end{aligned}
$$

where $T_{i, j}$ is temperature in node $(i, j)$, L node axial height, $Q_{\text {fission,ij }}$ total fission heat of current node $(i, j), Q_{R, i j}$ total radiation heat of the node $(i, j), Q_{c, i j}$ total convective heat transfer of the node $(\mathrm{i}, \mathrm{j}), \bar{k}_{i j, j-1}$ average conductivity to account for heat transfer between node $(i, j)$ and node $(i, j-1),-\bar{k}_{i j, i-1}$ average conductivity to account for heat transfer between node $(i, j)$ and node $(i-1, j)$. 


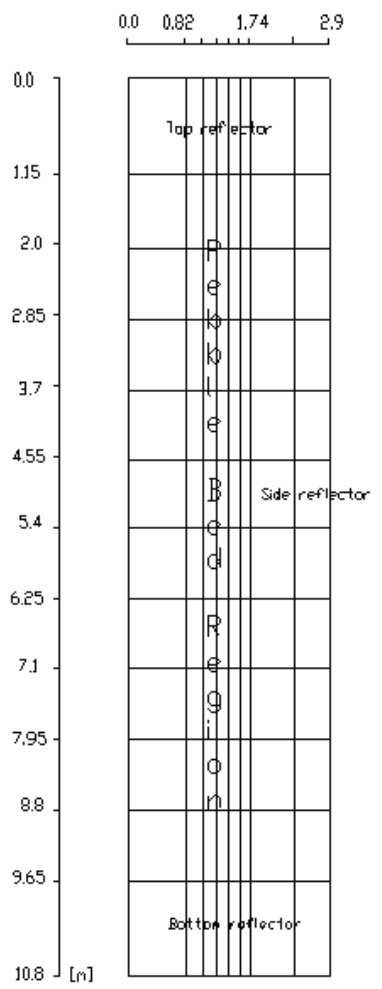

Figure 4.10 Core nodal scheme

Several constitutive relations and material properties must be provided to support the application of equation (4.37), i.e. the heat convection correlation between helium and pebbles, the effective pebble bed thermal conductivity and the thermal conductivity of graphite. The relations and properties used in the dynamic model are described in later sections.

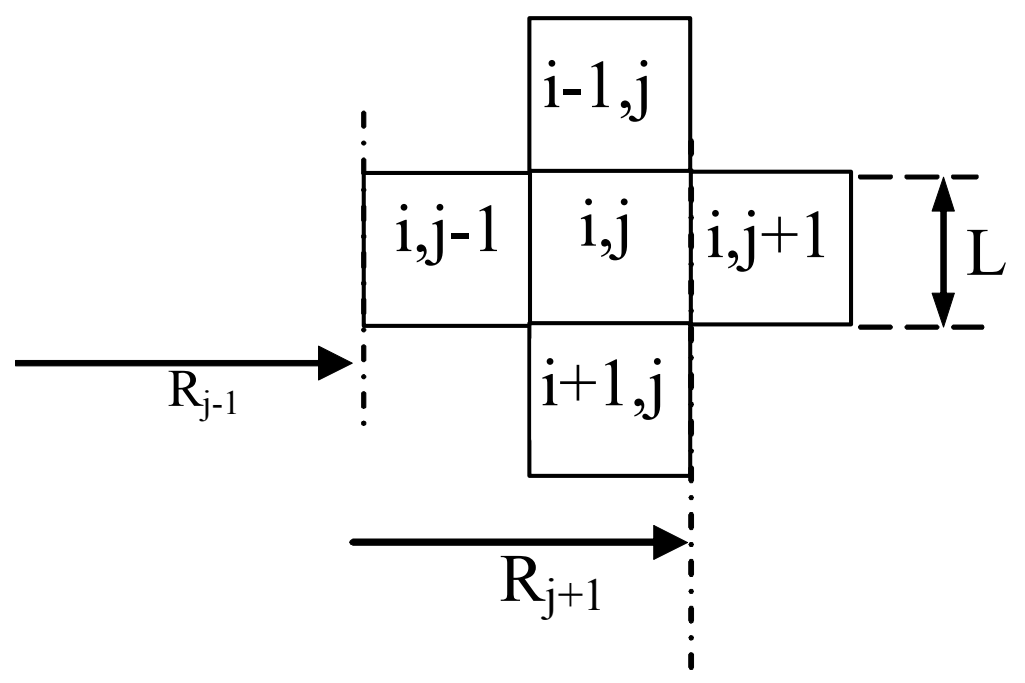

Figure 4.11 Nodes for core heat transport in cylindrical coordinates 


\section{- Correlation For Heat Convection}

In the pebble bed reactor, helium gas is used as coolant to remove heat from the core. As mentioned in the above, the helium flow in the radial direction is ignored. Thus, helium passes axially through the nodes of the pebble bed region. In the numerical treatment, it can be 1-dimensional, along the z-axis. In equation (4.38), $\mathrm{Q}_{\mathrm{C}, \mathrm{ij}}$ is used to account for the heat convection between helium and pebbles in a node. In numerical simulation, the node temperature represents the average pebble temperature. Therefore, the $\mathrm{Q}_{\mathrm{C}, \mathrm{ij}}$ for the node $(\mathrm{i}, \mathrm{j})$ can be determined by:

$Q_{C, i j}=\dot{m}_{g} C_{p g}\left(T_{g, \text { out }}-T_{g, \text { in }}\right)$

where $\dot{m}_{g}$ is the helium mass flow rate in a node, $\mathrm{T}_{\mathrm{g} \text {,out }}$ the helium outlet temperature and $\mathrm{T}_{\mathrm{g}, \text { in }}$ the helium inlet temperature in the node $(\mathrm{i}, \mathrm{j})$ and $\mathrm{C}_{\mathrm{pg}}$ the helium specific heat.

In a node, at the condition that fuel temperature distribution and helium inlet temperature are known, the helium outlet temperature can be derived from Figure 4.12 as follows:

In an infinitely small axial length of a node, the heat equilibrium yields

$\dot{m}_{g} C_{p g} d T_{g}(z)=h L_{\theta} d z\left(T_{f}-T_{g}(z)\right)$

Being arranged, equation (4.40) becomes

$d T_{g}(z)=\frac{h L_{\theta}}{\dot{m}_{g} C_{p g}} d z\left(T_{f}-T_{g}(z)\right)=a\left(T_{f}-T_{g}(z)\right) d z$

Then it becomes

$d\left[\exp (a z) T_{g}\right]=d\left[T_{f} \exp (a z)\right]$

Integrating equation (4.42) over the whole axial length of the node produces

$T_{g, \text { out }}=T_{g, \text { in }}+\left(T_{f}-T_{g, \text { in }}\right)\left[1-\exp \left(-\frac{h A_{c}}{\dot{m}_{g} C_{p g}}\right)\right]$ 
where $\dot{m}_{g}$ is helium mass flow rate $(\mathrm{kg} / \mathrm{s})$ in a node, $\mathrm{C}_{\mathrm{pg}}$ helium heat specific $(\mathrm{J} / \mathrm{kg} \mathrm{K}), \mathrm{T}_{\mathrm{f}}$ fuel average temperature in a node $(K), T_{g}$,in helium inlet temperature in a node $(K), T_{g, \text { out }}$ helium outlet temperature in a node $(\mathrm{K}), \mathrm{L}_{\theta}$ heat transfer circumferential length $(\mathrm{m}), \mathrm{L}$ axial length of a node $(\mathrm{m}), \mathrm{A}_{\mathrm{c}}$ the total heat transfer area of a node $\left(\mathrm{m}^{2}\right)$, and $\mathrm{h}$ the convective heat transfer coefficient developed by KFA based on experimental data from several independent studies of heat convection in pebble beds [15]:
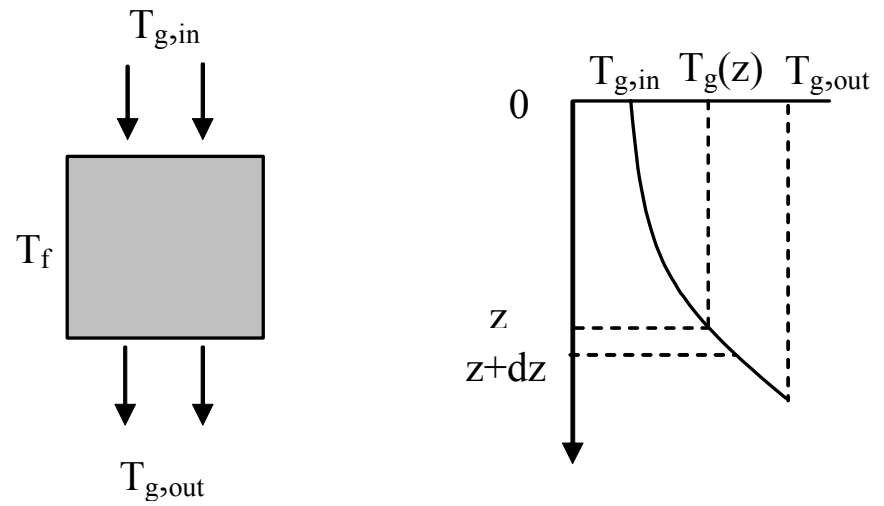

Figure 4.12 Schematic of helium and fuel heat transfer in a node

$h=\frac{k}{d_{p}} N u$

where $\mathrm{k}$ is helium thermal conductivity $(\mathrm{W} / \mathrm{m} \mathrm{K})$, and $\mathrm{d}_{\mathrm{p}}$ fuel pebble diameter $(\mathrm{m})$.

Here, $\mathrm{Nu}$ is determined by

$N u=1.27 \frac{\operatorname{Pr}^{0.333}}{\varepsilon^{1.18}} \operatorname{Re}^{0.36}+0.033 \frac{\operatorname{Pr}^{0.5}}{\varepsilon^{1.07}} \operatorname{Re}^{0.86}$,

where the Reynolds Number, Re, is calculated by

$\operatorname{Re}=\frac{\dot{m} d_{p}}{A_{C} \mu}$ 
and $\mathrm{d}_{\mathrm{p}}$ is pebble diameter $(\mathrm{m}), \dot{m}$ mass flow rate $(\mathrm{kg} / \mathrm{s}), \mathrm{A}_{\mathrm{c}}$ empty-core cross-sectional area, $\left(\mathrm{m}^{2}\right), \mu$ helium viscosity $\left(\mathrm{N}-\mathrm{s} / \mathrm{m}^{2}\right)$, Pr the helium Prandtl number, and $\varepsilon$ the void fraction of the pebble bed.

The application range of the above convective heat transfer coefficient is

$$
\begin{aligned}
& 100<\operatorname{Re}<10^{5}, \\
& 0.36<\varepsilon<0.42, \\
& D / d_{p}>20 \text { ( } D \text { is core diameter), } \\
& H>4 d_{p} \text { (H is mean core height). }
\end{aligned}
$$

All the above conditions are satisfied for the MPBR core.

\section{- Physical Model And Properties}

As the above equations show, we must know the pebble bed effective thermal conductivity and other material properties to calculate the heat transfer of the reactor core.

\section{Pebble Bed Effective Thermal Conductivity}

The heat transfer within the pebbles in the pebble bed involves both heat conduction through pebbles and heat radiation through the voids between pebbles. The helium conduction in the voids between pebbles is negligible, and the heat convection between helium and pebbles is modeled separately. Zehner and Schlunder used a cell model to determine the effective thermal conductivity taking into account heat conduction and radiation. Breitbach and Barthels developed a modified Zehner-Schlunder model which had been validated with experimental date[16]. Their relation of effective thermal conductivity depends on the temperature and neutron fluence.

General Electric developed a correlation for the pebble bed thermal conductivity which depends only on the temperature[17]:

$$
k(T)=1.1536 \times 10^{-4}(T-173.16)^{1.6622}
$$

$\mathrm{k}(\mathrm{T})$ is the thermal conductivity of the pebble bed $(\mathrm{W} / \mathrm{m} \mathrm{K})$, and $\mathrm{T}$ is temperature $(\mathrm{K})$. In the dynamic model, the above equation is adopted.

\section{Properties: Heat Capacity Of Pebble Bed}


For the pebble bed, the heat capacity can be determined by the correlation given by[18]:

$$
\rho C_{p}(T)=1.75 \times 10^{6} \times(1-\varepsilon)\left[0.645+3.14\left(\frac{T-T_{0}}{1000}\right)-2.809\left(\frac{T-T_{0}}{1000}\right)^{2}+0.959\left(\frac{T-T_{0}}{1000}\right)^{3}\right]
$$

where $\varepsilon$ is void fraction of pebble bed, $\mathrm{T}$ is node temperature $(\mathrm{K}), \mathrm{T}_{0}$ is reference temperature $(273.16 \mathrm{~K})$, and $\rho \mathrm{C}_{\mathrm{p}}$ is node heat capacity density $\left(\mathrm{J} / \mathrm{m}^{3} . \mathrm{K}\right)$.

\section{Reflector Properties}

The MPBR design will use graphite as the reflector. In the MPBR operating temperature range, the thermal conductivity of the reflector can be treated as roughly constant, $40 \mathrm{~W} / \mathrm{m}$ $\mathrm{K}$ [18]. The density is $1394.8 \mathrm{~kg} / \mathrm{m}^{3}$ [17]. The specific heat $\mathrm{C}_{\mathrm{p}}$ of the reflector as a function of temperature can be expressed as[19,20]:

$C_{p}=0.42 \times T+1500(\mathrm{~J} / \mathrm{Kg} \mathrm{K})$

where $\mathrm{T}$ is temperature $\mathrm{K}$.

The specific heat and viscosity of helium are required for calculating heat convection in the core. They are listed in Appendix D[21] along with other major helium thermal hydraulic and transport properties that will frequently be used throughout the modeling.

\section{- Point Kinetics Model}

The critical condition in a reactor represents a delicate dynamic balance between the neutron creation rate and neutron destruction rate. Reactor kinetics is the area of reactor physics devoted to predicting what happens to the neutron flux density when the balance condition associated with the critical condition is disturbed. The neutron production includes the prompt neutrons and the delayed neutrons. The prompt neutrons are generated at the moment of fission while the delayed neutrons are given off during the decay of the delayed neutron precursor nuclei. In reactor kinetics, the precursors can be represented by six groups of precursors. By assuming the shape of the neutron flux density is time-independent, which means that any local perturbation of the neutron flux density will spread throughout the reactor instantly, the core neutronics can be represented by the point kinetics equations describing the time-dependent behavior of the neutron flux density in the core. After assuming the one-speed diffusion approximation, the point kinetics equations with six groups of delayed precursors are [22]: 


$$
\begin{aligned}
& \frac{d \phi}{d t}=\frac{\rho-\beta}{\Lambda} \phi+v \sum_{i=1}^{6} \lambda_{i} C_{i}+v S \\
& \frac{d C_{i}}{d t}=\frac{\beta_{i}}{\Lambda v} \phi-\lambda_{i} C_{i} \quad(\mathrm{i}=1, \ldots, 6)
\end{aligned}
$$

where $\phi$ is core spatial and average neutron flux density, $\mathrm{C}_{\mathrm{i}}$ is concentration of delayed precursors of group $i, \lambda_{i}$ is decay constant of delayed precursors of group $i, \beta_{i}=$ effective delayed-neutron fraction for group i, $\mathrm{S}$ is neutron source, $v$ is neutron speed corresponding to energy E, $\rho$ is reactivity of the reactor, $\Lambda$ is prompt-neutron life time and the sum $\beta$ is the total effective delayed-neutron fraction:

$$
\beta \equiv \sum_{i=1}^{6} \beta_{i}
$$

The delayed neutron data are given in Table 4-2.

The general definition of reactivity can be found in [22]. Here we reduce it to

$$
\rho(t)=1-\frac{1}{k_{e f f}}
$$

where $k_{\text {eff }}$ is the effective multiplication factor. The reactivity of the reactor is the sum of the reactivity contributions by the core temperature feedback, reactivity control system, fission product poisoning and any external disturbance of reactivity. Here, reactivity change accounts for that of control system induced $\Delta \rho_{C S}$, temperature induced $\Delta \rho_{T}$ and xenon induced $\Delta \rho_{X}$ :

$$
\rho(t)=\Delta \rho_{C S}(t)+\Delta \rho_{T}(t)+\Delta \rho_{X}(t)
$$

The total fission power $\mathrm{Q}_{\mathrm{T}}(\mathrm{t})$ is related to the average neutron flux density $\phi(\mathrm{t})$ :

$$
Q_{T}(t)=\omega_{f} V \Sigma_{f} \phi(t)
$$

where $\omega_{\mathrm{f}}$ is the usable energy released per fission event, $\mathrm{V}$ is the active core volume and $\Sigma_{\mathrm{f}}$ is the macroscopic fission cross section, $\mathrm{m}^{-1}$ defined as

$$
\Sigma_{f}=N \sigma_{f}
$$

where $\mathrm{N}$ is the number of fissile nuclei $/ \mathrm{m}^{3}$ and $\sigma_{\mathrm{f}}$ is the microscopic cross section $\mathrm{m}^{2}$. In the nominal operating condition, the MPBR average neutron flux density is approximately $10^{14}$ $\mathrm{n} / \mathrm{cm}^{2} \mathrm{~s}[23]$. 
In equation (4.50), the average neutron flux $\phi$ can be replaced by the fission power $\mathrm{Q}_{\mathrm{T}}$. After some algebraic manipulations of equations (4.50) with (4.54), equation (4.50) becomes

$\frac{d Q_{T}}{d t}=\frac{\rho-\beta}{\Lambda} Q_{T}+\sum_{i=1}^{6} \lambda_{i} C_{i}+S$
$\frac{d C_{i}}{d t}=\frac{\beta_{i}}{\Lambda} Q_{T}-\lambda_{i} C_{i} \quad(\mathrm{i}=1, \ldots, 6)$

Note that the precursor concentration $\mathrm{C}_{\mathrm{i}}$ has been modified from the old one in equation (4.50), $\mathrm{C}_{\mathrm{i}, \text { new }}=\omega_{\mathrm{f}} \Sigma_{\mathrm{f}} \mathrm{UVC} \mathrm{C}_{\mathrm{i}, \text { old }}$, and the neutron source $\mathrm{S}_{\text {new }}=\omega_{\mathrm{f}} \Sigma_{\mathrm{f}} \mathrm{uVS} \mathrm{S}_{\text {old }}$.

In the current dynamic model, for simplification, one effective delayed group is used in the calculations rather than attempting to solve six delayed group equations. An average decay constant represents all delayed neutrons:

$\lambda=\left[\frac{1}{\beta} \sum_{i=1}^{6} \frac{\beta_{i}}{\lambda_{i}}\right]^{-1}$

and the one-effective-delayed-group point kinetics equations become:

$$
\begin{aligned}
& \frac{d Q_{T}}{d t}=\frac{\rho-\beta}{\Lambda} Q_{T}+\lambda C+S \\
& \frac{d C}{d t}=\frac{\beta}{\Lambda} Q_{T}-\lambda C
\end{aligned}
$$

These are a set of two coupled ordinary differential equations to represent the transient behavior of the thermal power and the decay of the delayed precursors.

It needs to be emphasized that the basis of the point kinetics equation derivation is timeindependence of the power distribution, which means the power distribution is fixed during any transient and steady state; the normalized fission power distribution is shown in Table 4.3.

\section{- Reactivity Model}

The reactivity model deals with the reactivity induced by fission product poisoning and caused by temperature variation.

\section{Fission Product Poisoning}

From the viewpoint of criticality and control, the most important fission product poison is $\mathrm{Xe}^{135}$ because of its large absorption cross section of about $2.65 \times 10^{6}$ barns at $0.025 \mathrm{eV}$ and its relatively large fission yield. The current model only considers the absorption of $\mathrm{Xe}^{135}$. The time-dependent behavior of $\mathrm{Xe}^{135}$ poisoning during transients is modeled as follows. 
$\mathrm{Xe}^{135}$ can be produced not only directly as a fission product but also by the $\beta$-decay of $\mathrm{Te}^{135}$. The relevant part of the fission chain is shown in Figure 4.13. The decay of $\mathrm{Te}^{135}$ is fast $(19.2 \mathrm{sec})$, and that of $\mathrm{Cs}^{135}$ is slow (2.6-million-year half-life). Therefore the rather complicated scheme can considerably simplified by assuming that $\mathrm{I}^{135}$ is formed directly from fission and the chain ends with the destruction by $\beta$ decay or neutron absorption of $\mathrm{Xe}^{135}$. The decay scheme is shown Figure 4.14. The time dependence of the concentrations of $\mathrm{I}^{135}$ and $\mathrm{Xe}^{135}$ based on the simplified scheme may be written[22]:

Table 4.2 Reactor core neutronic data [1]

\begin{tabular}{|l|l|l|}
\hline \multicolumn{2}{|l|}{ Prompt-neutron life time: $4.0 \times 10^{-4} \mathrm{sec}$} \\
\hline Delayed neutron data: & $\beta_{\mathrm{i}}$ & $\lambda_{\mathrm{i}}$ \\
\hline Group \# & $2 \times 10^{-4}$ & 0.01 \\
\hline 1 & $1 \times 10^{-3}$ & 0.02 \\
\hline 2 & $1 \times 10^{-3}$ & 0.12 \\
\hline 3 & $2 \times 10^{-3}$ & 0.30 \\
\hline 4 & $6 \times 10^{-4}$ & 1.10 \\
\hline 5 & $2 \times 10^{-4}$ & 2.90 \\
\hline 6 & & \\
\hline
\end{tabular}

Table 4.3 Normalized fission power distribution in pebble bed region

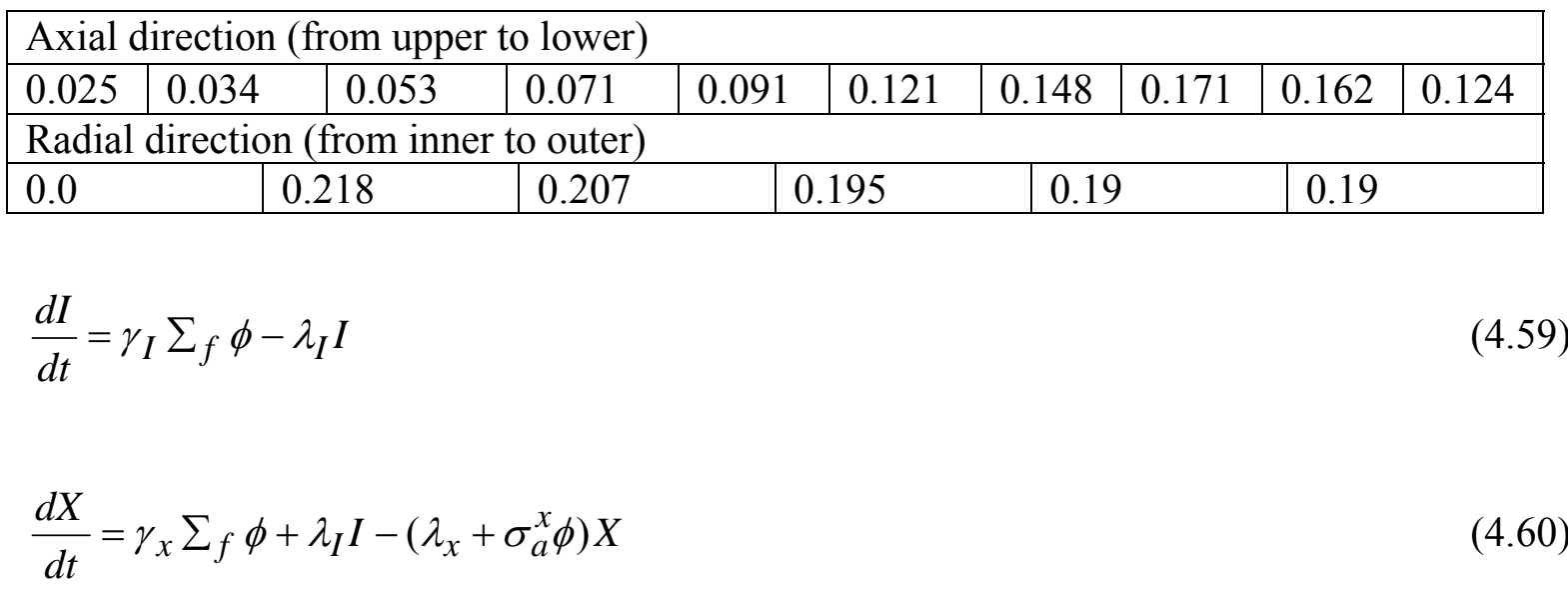

where $\mathrm{I}$ is ${ }^{135} \mathrm{I}$ concentration, $\mathrm{X}$ is ${ }^{135} \mathrm{Xe}$ concentration, $\phi$ is neutron flux, $\gamma_{I}, \gamma_{X}$ are effective fractions of ${ }^{135} \mathrm{I}$ and ${ }^{135} \mathrm{Xe}, \lambda_{I}, \lambda_{X}$ are $\beta$ decay constant of ${ }^{135} \mathrm{I}$ and ${ }^{135} \mathrm{Xe}, \Sigma_{f}$ is macroscopic fission section, and $\sigma_{a}^{X}$ is the microscopic absorption cross section of ${ }^{135} \mathrm{Xe}$.

The effective fraction and decay constants of $\mathrm{I}^{135}$ and $\mathrm{Xe}^{135}$ are given in Table 4.4. The reactivity that the ${ }^{135} \mathrm{Xe}$ concentration contributes during reactor operation is given by: 
$\Delta \rho=-\frac{\sum_{a}^{X}}{\sum_{a}}=-\frac{\sigma_{a}^{X} X}{\sum_{a}}$

At steady state, the concentrations of $\mathrm{I}^{135}, \mathrm{Xe}^{135}$ and the equilibrium reactivity due to the $\mathrm{Xe}^{135}$ concentration can be obtained from equations (4.59) and (4.60) setting both left sides to be equal to zero. For neutron flux $\phi(0)$ :

$$
\begin{aligned}
& I(0)=\frac{\gamma_{I} \Sigma_{f}}{\lambda_{I}} \phi(0) \\
& X(0)=\frac{\gamma_{X}+\gamma_{I}}{\lambda_{X}+\sigma_{a}^{X} \phi(0)} \Sigma_{f} \phi(0) \\
& \Delta \rho=-\frac{\sigma_{a}^{X}}{\Sigma_{a}} X(0)
\end{aligned}
$$

Since the decay $\mathrm{I}^{135}$ is much slow (6.58 hr half-life) compared to the load transients in the PCU, the fission product poisoning will not greatly affect the reactor characteristics in the timeframe of the interest in this study. However, the calculation for the Xe poisoning is still included in the dynamic model for the future long-term dynamic studies.

Table 4.4 Fission product fractions and decay constants

\begin{tabular}{|l|l|l|}
\hline & $\mathrm{I}^{135}$ & $\mathrm{Xe}^{135}$ \\
\hline Fission Product Fraction $\gamma(\%)$ & 6.386 & 0.288 \\
\hline$\beta$ - decay constant $\lambda(1 / \mathrm{s})$ & $2.87 \times 10^{-5}$ & $2.09 \times 10^{-5}$ \\
\hline
\end{tabular}

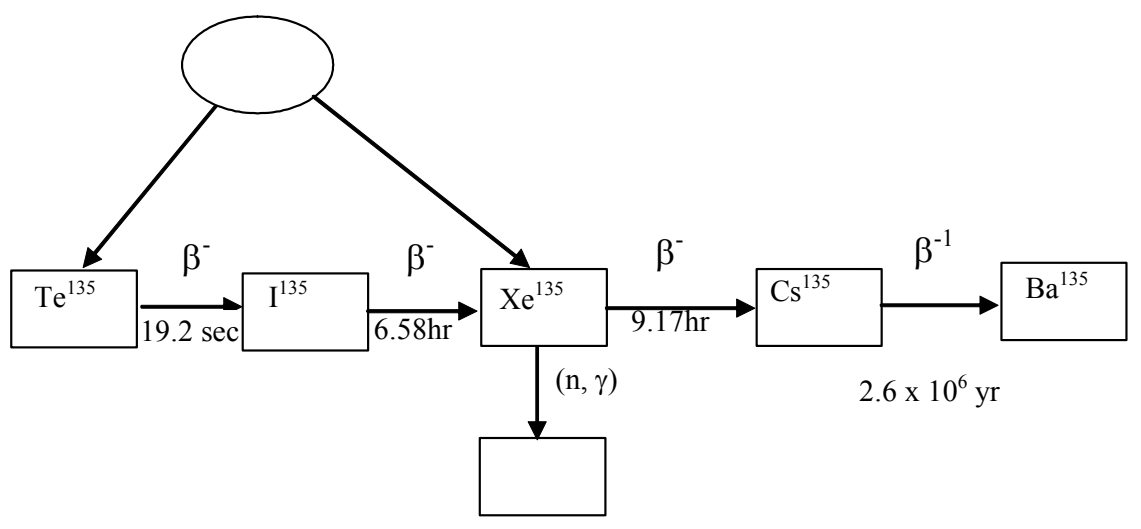

Figure 4.13 $\mathrm{Xe}^{135}$ fission-product chain [22] 


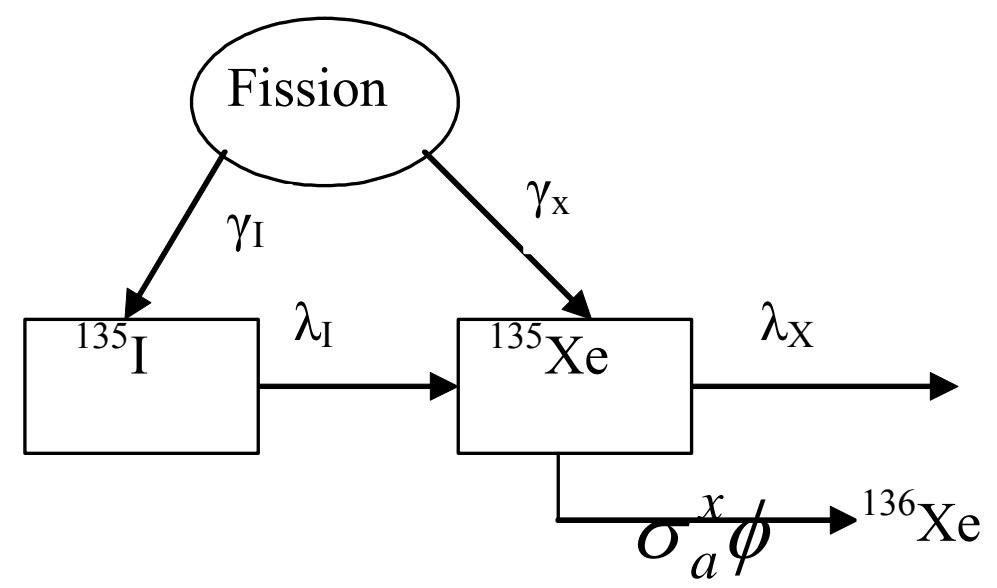

Figure 4.14 The simplified decay scheme of $\mathrm{Xe}^{135}$

\section{- Temperature Coefficients Of Reactivity}

As the core temperatures change, the other terms will change correspondingly, such as neutron spectrum and microscopic cross sections. Therefore, the reactivity will change as the temperatures of materials in the core vary. The temperature coefficient of reactivity may be defined as the rate of reactivity change per unit temperature change:

$\alpha_{T}=\frac{\partial \rho_{T}}{\partial T}$

Integrating over the temperature, we can get the reactivity change $\Delta \rho_{T}$ due to a temperature change $\Delta T$ :

$$
\Delta \rho_{T}(\Delta T)=\bar{\alpha}_{T} \Delta T
$$

A negative temperature coefficient leads to an inherently safe reactor. Increasing the core temperature induces a decrease of the effective multiplication constant, and then the power of the reactor power will decrease. Therefore, the core temperature will go down to the original values. In the same way, as the core temperature decreases, the effective multiplication constant increases. As a result, the reactor power tends to increase and the core temperature will increase as well. On the contrary, if the temperature coefficient is positive, the reactor is unstable and any small disturbance induces its amplification.

Since a detailed MBPR core design has not been performed, the temperature coefficient of reactivity is adopted as $-3.5 \times 10^{-5}[24]$, which is a rough estimation. The average temperature of the core is employed to define the coefficient 


\section{- Reactor Model Verification}

The reactor model can be verified with the results of other stand-alone calculations. Also, the sub-models can be verified with their analytical results, if such exist. The sub-model verifications with their analytical results are described as follows:

\section{Point Kinetics Equations Verification}

For one effective delayed-neutron group point kinetics, the analytical solutions can be obtained with S set to zero (no external source). It is usually assumed that the exponential form is the general solution of equation (4.56)

$$
Q_{T}(t)=Q_{T}(0) e^{\omega t}, C(t)=C(0) e^{\omega t}
$$

where $\omega$ are the eigenvalues of the coupled differential equations, and $\mathrm{Q}_{\mathrm{T}}(0)$ and $\mathrm{C}(0)$ are the initial conditions. The general solutions are (For detailed derivation of analytical solution see [22] Section 7):

$$
\begin{aligned}
& \frac{Q_{T}(t)}{Q_{T}(0)} \approx \frac{-\rho}{\beta-\rho} \exp \left(-\frac{\beta-\rho}{\Lambda} t\right)+\frac{\beta}{\beta-\rho} \exp \left(\frac{\lambda \rho}{\beta-\rho} t\right) \\
& \frac{C(t)}{C(0)} \approx \frac{\rho \beta}{(\beta-\rho)^{2}} \exp \left(-\frac{\beta-\rho}{\Lambda} t\right)+\frac{\beta}{\Lambda \lambda} \exp \left(\frac{\lambda \rho}{\beta-\rho} t\right)
\end{aligned}
$$

By using $\Lambda=4.0 \times 10^{-4} \mathrm{sec}, \beta=5.0 \times 10^{-3}$ and $\lambda=5.84 \times 10^{-2} \mathrm{sec}^{-1}$, the analytical results and model numerical results are obtained for both positive and negative reactivity insertion of amount $|\rho|=0.22 \%$. The results are shown in Figure 4.15.From the figure, the results of analytical and numerical calculation for negative reactivity insertion essentially coincide. For positive reactivity insertion, the numerical results are slightly higher than the analytical ones; the calculation error is caused by use of a relatively large time step size in simulation.

\section{Fission Product Poisoning Verification}

The reactivity generated by fission product buildup has a significant impact on the adjusting of the control rods in the control of reactor startup after reactor temporary shutdown.

Therefore, it is important to predict the fission product poisoning for control processes. A test simulation was performed to verify the $\mathrm{Xe}^{135}$ buildup after the reactor shutdown. The results are shown in Figure 4.16.

When the reactor is shut down to $\phi=0$ after operating under equilibrium conditions with the neutron flux $\phi(0)$, the concentrations of $\mathrm{I}^{135}$ and $\mathrm{Xe}^{135}$ can be obtained from equations (4.59) and (4.60) [22]: 


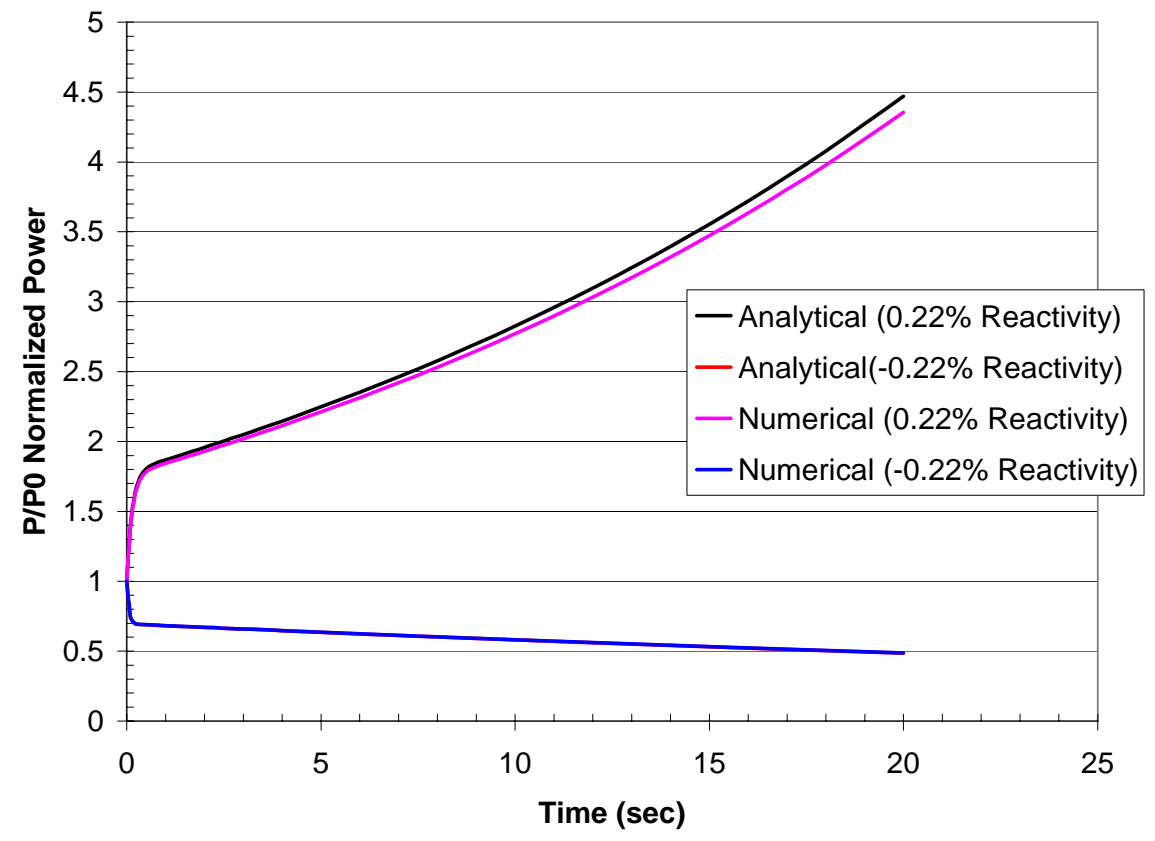

$I(t)=\frac{\gamma_{I} \Sigma_{f} \phi(0)}{\lambda_{I}} \exp \left(-\lambda_{I} t\right)$
$X(t)=\frac{\gamma_{f} \phi(0)}{\sigma_{a}^{X} \phi(0)+\lambda_{X}} \exp \left(-\lambda_{X} t\right)+\frac{\gamma_{I} \Sigma_{f} \phi(0)}{\lambda_{I}-\lambda_{X}}\left[\exp \left(-\lambda_{X} t\right)-\exp \left(-\lambda_{I} t\right)\right]$

where $\gamma=\gamma_{I}+\gamma_{X}$

The time that the concentration of $\mathrm{Xe}^{135}$ reaches maximum is

$t_{\max }=\frac{1}{\lambda_{I}-\lambda_{X}} \ln \left[\frac{\lambda_{I} / \lambda_{X}}{1+\frac{\lambda_{X}}{\lambda_{I}}\left(\frac{\lambda_{I}}{\lambda_{X}}-1\right) \frac{X(0)}{I(0)}}\right]$

When $\phi(0)=10^{18} \mathrm{n} /\left(\mathrm{m}^{2} \mathrm{~s}\right)$, the time predicted by the numerical solution is $10.5 \mathrm{hr}$ as shown in Figure 4.16.

Figure 4.15 Numerical results versus analytical results for one effective group point kinetics equations 


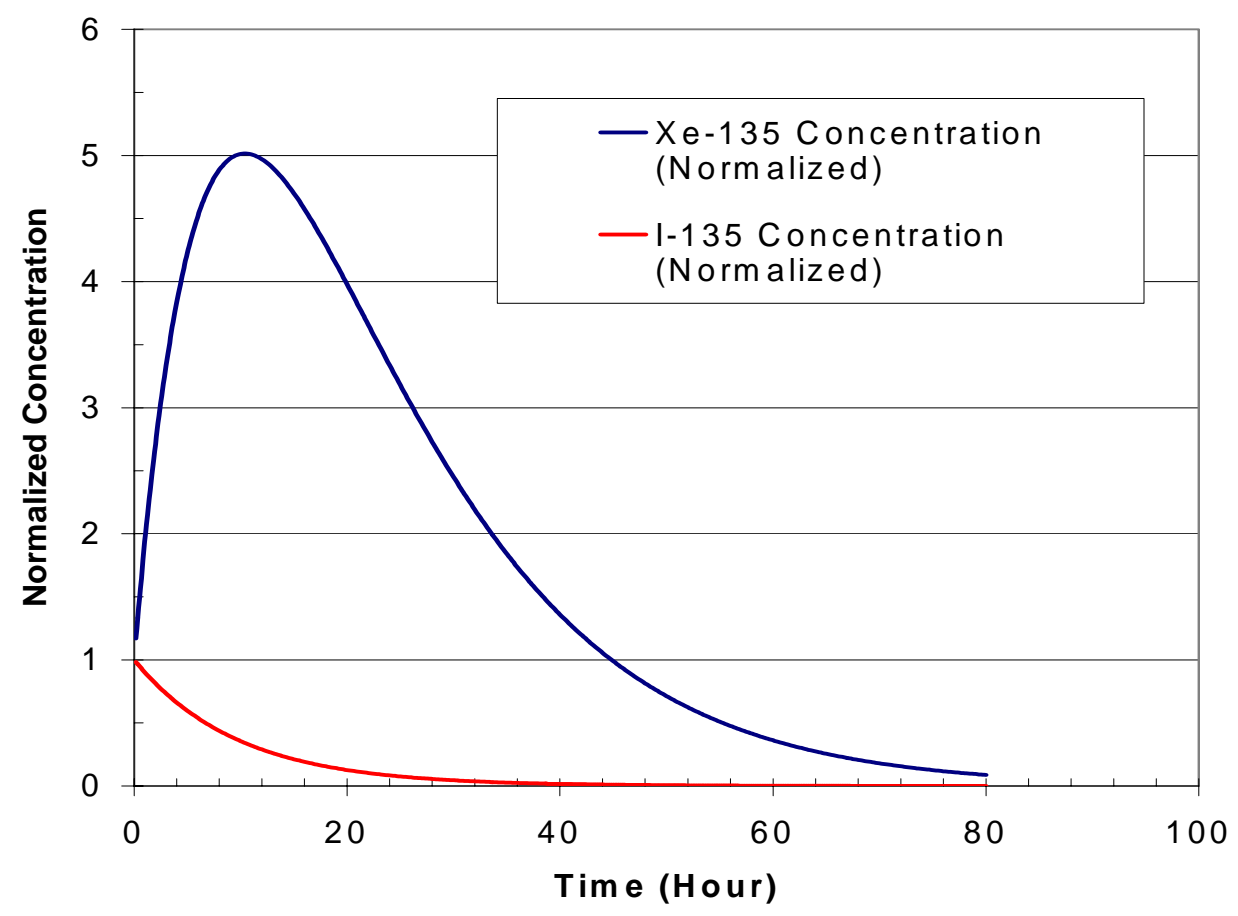

Figure 4.16 $\mathrm{Xe}^{135}$ buildup following core shutdown

\subsubsection{Heat Exchanger Model}

In the MPBR design, heat exchangers are incorporated in the cycle to improve the cycle performance. The IHX and recuperator are helium/helium heat exchangers while the precooler and intercoolers are helium/water units. In the preliminary design, the IHX will use counterflow plat-fine or the printed circuit configurations and the recuperator will be of the counterflow plate-fin type. The precooler and intercoolers are expected to be shell-tube configurations. The characteristics of the heat exchangers remarkably affect the overall transient response to changes of the operating variables. Hence, a reliable dynamic model for predicting the performance of a heat exchanger is necessary.

Helium is the working fluid both in the hot side and cold side in the IHX and recuperator. In the precoolers and intercoolers, the helium is the working fluid in the hot side, and water is in the cold side. In the cold side, the highest water temperature is designed to be much lower than the water boiling point, so that water remains single phase in the precooler and intercoolers.

Temperature changes which occur in the inlet of the cold side of a heat exchanger do not affect the hot side outlet temperature instantaneously. The lag is determined by the thermal capacitances of the wall metal and the working fluids as well as the resistances to heat transfer. The dynamic model solves the governing equations based on energy conservation. 
Some simplifying assumptions are made in order to obtain relations that can be solved efficiently. The major assumptions are listed as follows:

1. The heat exchanger is adiabatic. This means that the heat exchanger is insulated from the outside surroundings and there is no heat transfer to the environment.

2. The weight of the side wall is small in comparison with the amount of weight of the plates separating the flow passages. The side wall is ignored.

3. Heat conduction in the longitudinal direction is negligible in both the fluid and the solid walls.

4. For both fluid and solid wall, the temperature only varies in the flow direction. This implies that the temperature is only the function of $x$ position (shown in Figure 4.17) while the $y, z$ positions do not affect the temperature.

\section{Nodal Scheme}

A counterflow heat exchanger can be treated as three regions: the hot fluid side, metal wall and cold fluid side. In the longitudinal direction, it is divided into a number of sections; each section is a differential element (node) of the heat exchanger. This method is called the lumped parameter modeling approach, which also is used in many engineering transient analyses for heat exchangers[25]. The nodal scheme is shown in Figure 4.17. As mentioned above, there are three regions in a differential element, and the temperature in each region is uniform. The hot fluid temperature is $\mathrm{T}_{\mathrm{c}}$, mass flowrate is $\dot{m}_{h}$; the separating wall temperature is $\mathrm{T}_{\mathrm{m}}$; the cold fluid temperature is $\mathrm{T}_{\mathrm{c}}$ and mass flowrate is $\dot{m}_{C}$. We use $\mathrm{U}_{\mathrm{h}}$ to denote the overall conductance for the heat transfer between the hot fluid and the separating wall, and $U_{c}$ represents that for the cold fluid and the separating wall.

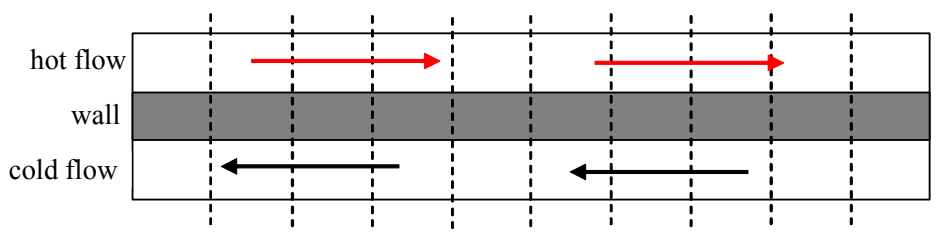

(a) Division of a Heat Exchanger

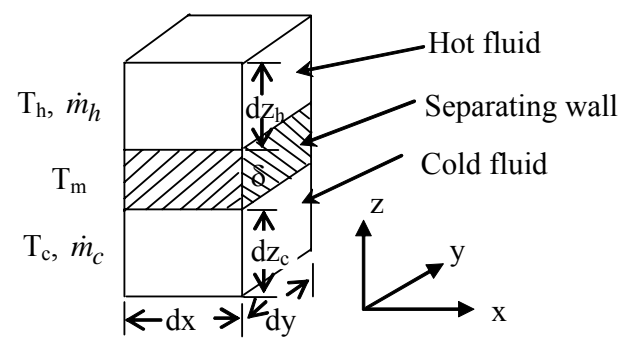

(b) Differential Element (Node)

Figure 4.17 Nodal scheme of a heat exchanger 


\section{Transient Differential Equations}

The transient equations for the model are derived basing on the energy balance for each region of the differential element presented in the above sketch.

For hot fluid:

$\rho_{h} d x d y d z_{h} C p_{h} \frac{\partial T_{h}}{\partial t}=-\left(T_{h, o}-T_{h, i}\right) \dot{m}_{h} C p_{h}+U_{h} d x d y\left(T_{m}-T_{h}\right)$

For separating wall:

$\rho_{m} d x d y \delta C p_{m} \frac{\partial T_{m}}{\partial t}=U_{h} d x d y\left(T_{h}-T_{m}\right)+U_{C} d x d y\left(T_{m}-T_{C}\right)$

For cold fluid:

$\rho_{C} d x d y d z_{C} C p_{C} \frac{\partial T_{C}}{\partial t}=-\left(T_{C, o}-T_{C, i}\right) \dot{m}_{C} C p_{C}+U_{C} d x d y\left(T_{m}-T_{C}\right)$

After arrangement, eqn. (4.69) can be reduced to:

$$
\begin{gathered}
\frac{\partial T_{h}}{\partial t}=\frac{1}{\tau_{h 1}}\left(T_{m}-T_{h}\right)+\frac{1}{\tau_{h 2}}\left(T_{h, i}-T_{h, o}\right) \\
\tau_{h 1}=\frac{\rho_{h} d z_{h} C p_{h}}{U_{h}}, \tau_{h 2}=\frac{\rho_{h} d x d y d z_{h}}{\dot{m}_{h}}
\end{gathered}
$$

eqn.(4.70) can be reduced to:

$$
\begin{gathered}
\frac{\partial T_{m}}{\partial t}=\frac{1}{\tau_{m 1}}\left(T_{h}-T_{m}\right)+\frac{1}{\tau_{m 2}}\left(T_{m}-T_{C}\right) \\
\tau_{m 1}=\frac{\rho_{m} \delta C p_{m}}{U_{h}}, \tau_{m 2}=\frac{\rho_{m} \delta C p_{m}}{U_{C}}
\end{gathered}
$$

eqn.(4.71) can be reduced to:

$$
\begin{aligned}
& \frac{\partial T_{C}}{\partial t}=\frac{1}{\tau_{c 1}}\left(T_{m}-T_{C}\right)+\frac{1}{\tau_{c 2}}\left(T_{C, i}-T_{C, o}\right) \\
& \tau_{h 1}=\frac{\rho_{C} d z_{C} C p_{C}}{U_{C}}, \tau_{h 2}=\frac{\rho_{C} d x d y d z_{C}}{\dot{m}_{C}}
\end{aligned}
$$

where

$\mathrm{t}=$ time, $\mathrm{s}$,

$\mathrm{dx}, \mathrm{dy}, \mathrm{dz}, \mathrm{dz} \mathrm{z}_{\mathrm{c}}=$ the spatial dimension of the hot fluid and cold fluid in the differential element; $\mathrm{m}$,

$\delta=$ the thickness of the separating wall, $\mathrm{m}$,

$\mathrm{T}_{\mathrm{h}}, \mathrm{T}_{\mathrm{c}}=$ the temperatures of the hot fluid and cold fluids, respectively, $\mathrm{K}$,

$\mathrm{T}_{\mathrm{h}, \mathrm{i}}, \mathrm{T}_{\mathrm{h}, \mathrm{o}}=$ the inlet and outlet temperature of the hot fluid region, $\mathrm{K}$,

$\mathrm{T}_{\mathrm{c}, \mathrm{i}}, \mathrm{T}_{\mathrm{c}, \mathrm{o}}=$ the inlet and outlet temperature of the cold fluid region, $\mathrm{K}$, 
$\mathrm{T}_{\mathrm{m}}=$ separating wall temperature, $\mathrm{K}$,

$\rho_{\mathrm{h}}, \rho_{\mathrm{c}}=$ the density of hot fluid and cold fluid, respectively, $\mathrm{kg} / \mathrm{m}^{3}$,

$\rho_{\mathrm{m}}=$ density of the separating wall, $\mathrm{kg} / \mathrm{m}^{3}$,

$\mathrm{Cp}, \mathrm{Cp}_{\mathrm{c}}=$ the specific heat of the hot fluid and cold fluid, respectively, $\mathrm{J} / \mathrm{kg} \mathrm{K}$,

$\mathrm{Cp}_{\mathrm{m}}=$ the specific heat of the separating wall, $\mathrm{J} / \mathrm{kg} \mathrm{K}$,

$\dot{m}_{h}, \dot{m}_{C}=$ the mass flowrate of the hot fluid and cold fluid, respectively, $\mathrm{kg} / \mathrm{s}$,

$\mathrm{U}_{\mathrm{h}}, \mathrm{U}_{\mathrm{c}}=$ the overall conductance of the heat transfer between the hot fluid and

separating wall and between the cold fluid and separating wall, respectively, $\mathrm{J} / \mathrm{m}^{2} \mathrm{~K}$,

$\tau_{\mathrm{h} 1}, \tau_{\mathrm{h} 2}, \tau_{\mathrm{c} 1}, \tau_{\mathrm{c} 2}, \tau_{\mathrm{m} 1}, \tau_{\mathrm{m} 2}=$ time constants, $\mathrm{s}$.

When the mass flowrate varies, the overall conductance also changes, as a result, the corresponding time constant $\tau$ varies. The variation of time constant $\tau$ responding to a change of the mass flowrate is influenced by the heat exchanger design. In Table 4.5 for the IHX design, there is the relation of the Reynolds number and $\mathrm{St}^{*} \mathrm{Pr}^{2 / 3}$, in which $\mathrm{St}$ is the Stanton number and Pr is the Prandtl number.

To simplify solving the differential equation, the energy balance for the helium neglects the short holdup times, thus:

$\frac{\partial T_{h}}{\partial t}=0$

so that eqn. (4.72) becomes:

$T_{h, i}-T_{h, o}=n_{h}\left(T_{h}-T_{m}\right)$

where $\mathrm{n}_{\mathrm{h}}$ is a constant for the hot side helium:

$n_{h}=\frac{U_{h} d x d y}{C p_{h} \dot{m}_{h}}$

$\mathrm{n}_{\mathrm{h}}$ also changes when the mass flowrate varies. As the length of the differential element increases, $n$ increases as well. If $n_{h}>2$, the initial response of $T_{h, o}$ to a step change in $T_{h, i}$ will be opposite in sign to the input change. It is nonphysical. The number of sections must be sufficient so that $\mathrm{n}<2$ at minimum expected flow rates[26].

\section{Verification}

Flownet is a general thermal-fluid network analysis code that can model the transient of a heat exchanger[27]. Comparisons have been made between the Flownet model and the model in this report for simulating a temperature transient of the IHX. The temperature transient is that fixing hot side inlet temperature, and helium mass flowrate in the hot side and cold side, and then the cold side inlet temperature step increases $100^{\circ} \mathrm{C}$ from $488.8^{\circ} \mathrm{C}$ at a prescribed time. The IHX is the Concept-NREC printed circuit design, and the design details are listed in Table 4.5. The IHX hot side outlet and cold side outlet temperatures of the two model results are shown in Figures 4.18 and 4.19, respectively. It can be seen that 
the transient characteristics depicted for the two models are in good agreement for the temperature transient.

\subsubsection{Turbomachinery Model}

The axial type is adopted as the configuration of all the turbines in the MPBR design. The axial turbine preliminary design has been conducted by Concepts NREC based upon experience and mean line analysis. The HP turbine and LP turbine are both four-stage axial ones. The power turbine is also an axial type and one preliminary and unconfirmed result shows it is 23 stages. For compressors, centrifugal type and axial type were both investigated by Concepts NREC. The aerodynamic design for a five-stage centrifugal compressor has been performed with nominal operating shaft speed of $5900 \mathrm{rpm}$ and its estimated performance has been obtained. Also, an axi-centrifugal compressor with eight axial stages and one centrifugal stage has been developed with rotational speed of $8000 \mathrm{rpm}$.

Table 4.5 IHX design details (Printed Circuit Configuration)

\begin{tabular}{|c|c|c|}
\hline Effectiveness, $\%$ & \multicolumn{2}{|l|}{95} \\
\hline Hot-side dP, \% & \multicolumn{2}{|l|}{1.77} \\
\hline Cold-side dP, \% & \multicolumn{2}{|l|}{2} \\
\hline Weight (kg) & \multicolumn{2}{|l|}{75,480} \\
\hline Metal specific heat $(\mathrm{J} / \mathrm{kg} \mathrm{K})$ & \multicolumn{2}{|l|}{460} \\
\hline Thermal cond. (W/m-K) & \multicolumn{2}{|l|}{18.69} \\
\hline Wall thickness & \multicolumn{2}{|l|}{$6 \mathrm{~mm}$} \\
\hline HT area hot side $\left(\mathrm{m}^{\wedge} 2\right)$ & \multicolumn{2}{|l|}{4262.4} \\
\hline HT area cold side $\left(\mathrm{m}^{\wedge} 2\right)$ & \multicolumn{2}{|l|}{4262.4} \\
\hline Length $(\mathrm{m})$ & \multicolumn{2}{|l|}{1.2548} \\
\hline Hydraulic Diam. (m) & \multicolumn{2}{|l|}{$1.13^{*} 10^{\wedge}-3$} \\
\hline UA (W/K) & \multicolumn{2}{|l|}{$1.0777^{*} 10^{\wedge} 7$} \\
\hline Reynolds No. hot side & \multicolumn{2}{|l|}{1,845} \\
\hline Reynolds No. cold side & \multicolumn{2}{|l|}{1,845} \\
\hline HT coeff hot side $\left(\mathrm{W} / \mathrm{m}^{\wedge} 2-\mathrm{K}\right)$ & \multicolumn{2}{|l|}{5735} \\
\hline HT coeff cold side $\left(\mathrm{W} / \mathrm{m}^{\wedge} 2-\mathrm{K}\right)$ & \multicolumn{2}{|l|}{5695} \\
\hline Flow area hot side $\left(\mathrm{m}^{\wedge} 2\right)$ & \\
\hline Flow area cold side $\left(\mathrm{m}^{\wedge} 2\right)$ & \multicolumn{2}{|l|}{1.542} \\
\hline $\operatorname{Re}$ & $\mathrm{f}$ (friction factor) & $\mathrm{j}\left(\mathrm{St}^{*} \mathrm{Pr}^{\wedge} 2 / 3\right)$ \\
\hline 300 & 0.1294 & 0.02266 \\
\hline 2000 & 0.06164 & 0.01174 \\
\hline 10000 & 0.03285 & 0.006722 \\
\hline
\end{tabular}




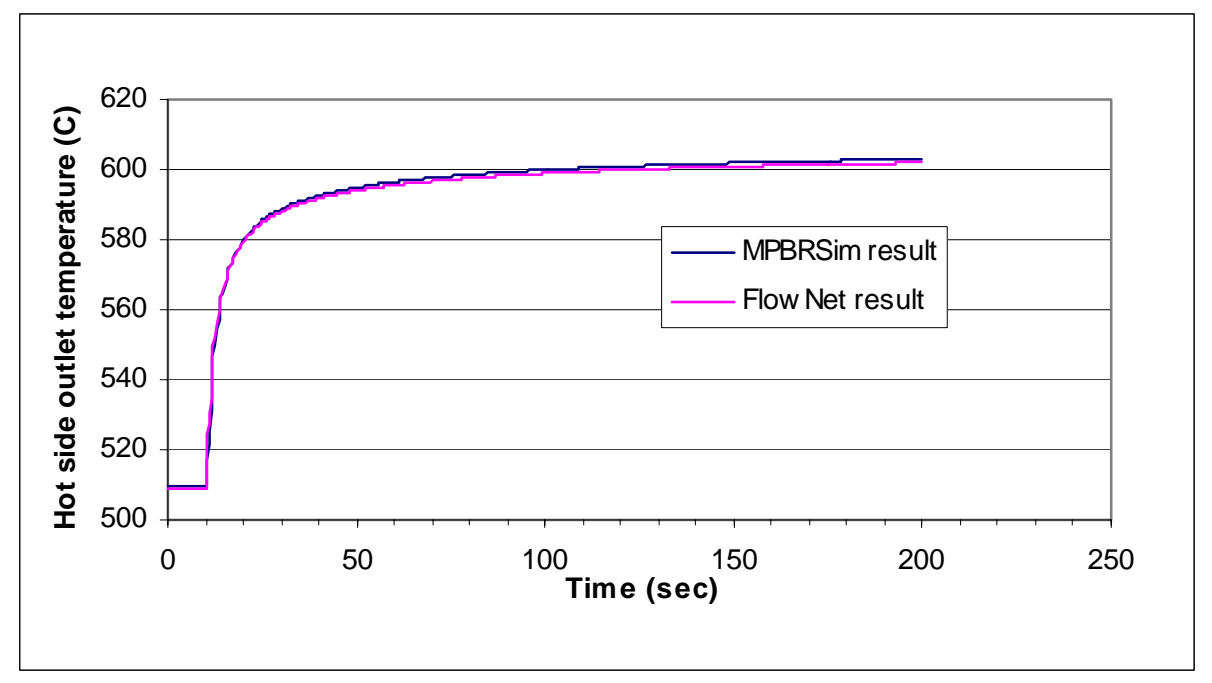

Figure 4.18 HX model verification: hot side outlet temperature response to cold side inlet temperature $100^{\circ} \mathrm{C}$ step increase at $10 \mathrm{sec}$

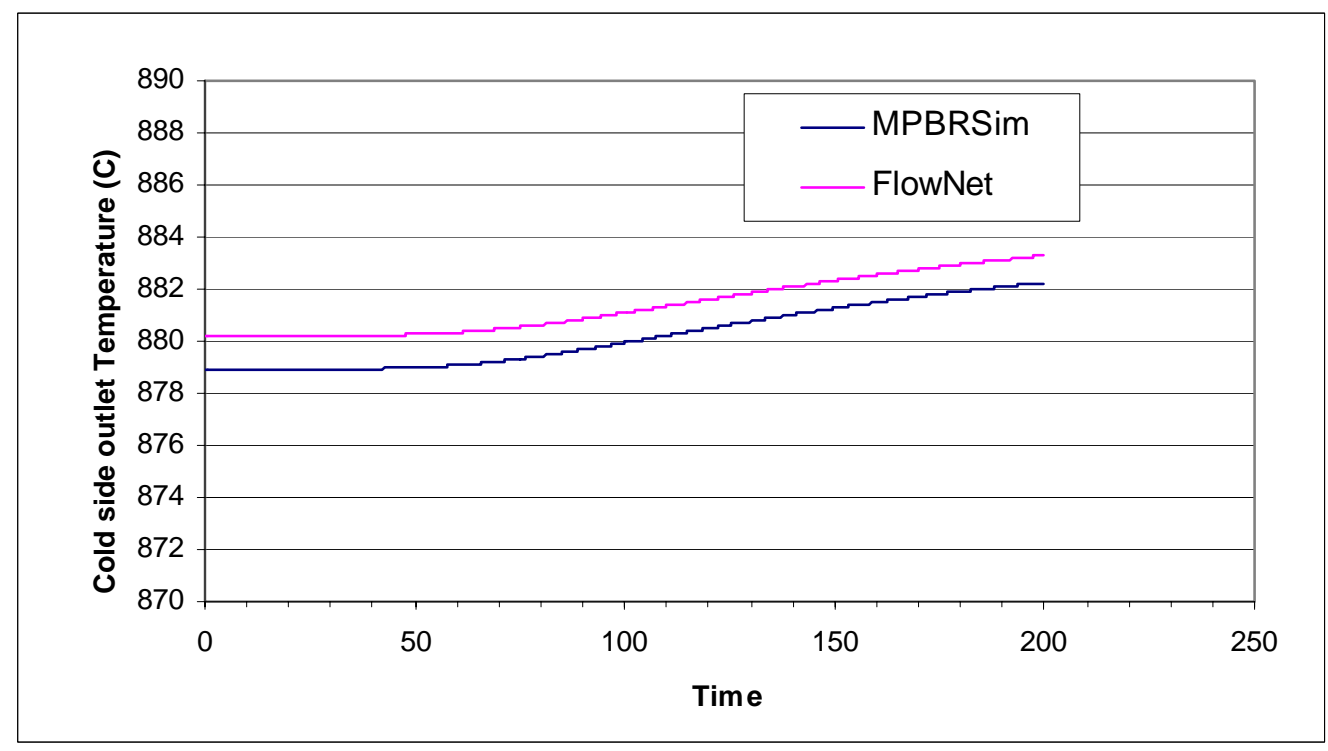

Figure 4.19 HX model verification: Cold side outlet temperature response to cold side inlet temperature $100^{\circ} \mathrm{C}$ step increase at $10 \mathrm{sec}$

After a turbomachine has been fabricated, its complete characteristic can be obtained by measuring the operating variables only if it runs in a real plant or a test rig. However, estimated characteristics can be obtained based on experience and numerical analysis after it has been designed. The overall characteristics of a gas turbine or gas compressor can be plotted by using a combination of parameters, i.e. flow coefficient, actual enthalpy rise coefficient and ideal enthalpy rise coefficient. If the parameters have been chosen appropriately, the speed lines of an axial turbine can be collapsed to a single line, which 
remarkably benefits the numerical convergence if it is used in the dynamic model to represent the turbine. The non-dimensional parameters used in this thesis are defined as follows:

Flow Coefficient $=\frac{\dot{m}_{c o r}}{\rho_{c o r} A U}$

Actual Enthalpy Rise Coefficient $=\frac{\Delta h}{U^{2}}$

Ideal Enthalpy Rise Coefficient $=\frac{\Delta h_{\text {ideal }}}{U^{2}}$

where $\dot{m}_{c o r}$ is the corrected mass flowrate, $\rho_{\text {cor }}$ is the corrected density, A is area, $U$ is the tip speed, $\Delta \mathrm{h}$ is actual enthalpy rise and $\Delta \mathrm{h}_{\text {ideal }}$ is the ideal enthalpy rise.

For a compressor:

$\eta_{i}=\Delta h_{\text {ideal }} / \Delta h$

and for a turbine:

$\eta_{i}=\Delta h / \Delta h_{\text {ideal }}$

where $\eta_{i}$ is the isentropic efficiency.

The power of the turbomachine is

$P=\dot{m} \cdot \Delta h$

and for a compressor

$\Delta \mathrm{h}=\mathrm{Cp}\left(\mathrm{T}_{2}-\mathrm{T}_{1}\right)$

for a turbine

$\Delta \mathrm{h}=\mathrm{Cp}\left(\mathrm{T}_{1}-\mathrm{T}_{2}\right)$

where $\dot{m}$ is the mass flowrate, $\mathrm{T}_{2}$ is the outlet temperature, $\mathrm{T}_{1}$ is the inlet temperature and $\mathrm{Cp}$ is helium specific heat. By coupling with the following equation, the outlet pressure can be obtained when the power and inlet conditions are known.

Knowing the inlet temperature, pressure ratio and isentropic efficiency, the outlet temperature can be calculated using Equation (4.10) for the compressor and Equation (4.12) for the turbine.

If using the above defined non-dimensional parameters, the geometry of the specific turbomachine needs to be known, i.e. the blade tip diameter for obtaining the tip speed. In order to avoid the need for the specific geometry values of the machine, the non-dimensional parameters are redefined in dimensional form. This can be achieved by using the machine 
operating conditions, such as using the rotational speed replaced by the tip speed. The parameters are redefined as follows:

Flow Coefficient $=\dot{m}_{\text {cor }} / N_{\text {cor }}$

Actual Enthalpy Rise Coefficient $=\Delta h / N_{\text {cor }}^{2}$

Ideal Enthalpy Rise Coeffiecient $=\Delta h_{\text {ideal }} / N_{\text {cor }}^{2}$

where $\dot{m}_{c o r}$ is the corrected mass flowrate defined by

$\dot{m}_{\text {cor }} \equiv \frac{\dot{m} \sqrt{T_{1}}}{p_{1}}$

and $\mathrm{N}_{\text {cor }}$ is the corrected speed defined by

$N_{\text {cor }} \equiv \frac{N}{\sqrt{T_{1}}}$

where $\mathrm{N}$ denotes the rotational speed. Tables 4.6 and 4.7 show the comparisons of the basic performance parameters, dimensionless parameters, and dimensioned parameters used in this study.

The overall plant dynamic model was developed for investigating the interaction of the components in transient condition and optimizing the plant cycle. The cycle arrangement and nominal operating parameters are variables in the design process. The dynamic model should be flexible enough to adapt cycle changes such as various shaft and heat exchanger arrangements. When plotting the characteristic, the above defined parameters are normalized to the their design point values. Though this method is not rigorous, this characterization of performance provides a reasonable and approximate means of defining performance at any power, flow or efficiency required.

The methodology used here is semi-rigorous and will generate errors with increasing magnitude when the pressure ratio, efficiency and flow or tip-speed increasingly deviate from the original design. The initial expectation is that the differences of the pressure ratio, efficiency and flow with the original design remain small. While curve shape and slope will change with the flow coefficient, these changes are expected to fall within the range of design variations seen from different manufacturers.

Figure 4.20 shows the normalized characteristic of the axial turbine. The normalized characteristic of the centrifugal compressor is shown in Figures 4.21 and 4.22. For the axial compressor, the performance is shown in Figures 4.23 and 4.24.

Using the turbomachine map in the dynamic model implies that the energy storage and transport delay in the turbine and compressor are small and negligible. 
Table 4.6 Compressor parameter definitions

\begin{tabular}{|l|c|c|c|}
\hline & Basic & $\begin{array}{c}\text { Dimensionless } \\
\text { coefficient }\end{array}$ & $\begin{array}{c}\text { Dimensioned } \\
\text { coefficient }\end{array}$ \\
\hline $\begin{array}{l}\text { Flow, } \\
\text { Flow coefficient }\end{array}$ & $\dot{m}$ & $\dot{m}_{\text {cor }} /\left(\rho_{\text {cor }} \cdot A \cdot U\right)$ & $\dot{m}_{\text {cor }} / N_{\text {cor }}$ \\
\hline $\begin{array}{l}\text { Power, } \\
\text { Work coefficient } \\
\text { Actual enthalpy rise coefficient }\end{array}$ & $P=\dot{m} \cdot \Delta h$ & $\Delta h / U_{2}^{2}$ & $\Delta h / N_{c o r}^{2}$ \\
\hline $\begin{array}{l}\text { Pressure ratio } \\
\text { Pressure coefficient } \\
\text { Ideal enthalpy rise coefficient }\end{array}$ & $\mathrm{PR}=\mathrm{p}_{2} / \mathrm{p}_{1}$ & $\Delta h_{\text {ideal }} / U_{2}^{2}$ & $\Delta h_{\text {Ideal }} / N_{c o r}^{2}$ \\
\hline $\begin{array}{l}\text { Isentropic efficiency } \\
\text { Polytropic efficiency(perfect } \\
\text { gas) }\end{array}$ & $\eta_{i}=\Delta h_{\text {Ideal }} / \Delta h$ & - & - \\
\hline
\end{tabular}

Table 4.7 Turbine parameter definitions

\begin{tabular}{|l|c|c|c|}
\hline & Basic & $\begin{array}{c}\text { Dimensionless } \\
\text { coefficient }\end{array}$ & $\begin{array}{c}\text { Dimensioned } \\
\text { coefficient }\end{array}$ \\
\hline $\begin{array}{l}\text { Flow, } \\
\text { Flow coefficient }\end{array}$ & $\dot{m}$ & $\dot{m}_{\text {cor }} /\left(\rho_{\text {cor }} \cdot A \cdot U\right)$ & $\dot{m}_{\text {cor }} / N_{\text {cor }}$ \\
\hline $\begin{array}{l}\text { Power, } \\
\text { Work coefficient } \\
\text { Actual enthalpy rise coefficient }\end{array}$ & $P=\dot{m} \cdot \Delta h$ & $\Delta h / U_{2}^{2}$ & $\Delta h / N_{\text {cor }}^{2}$ \\
\hline $\begin{array}{l}\text { Pressure ratio } \\
\begin{array}{l}\text { Pressure coefficient } \\
\text { Ideal enthalpy rise coefficient }\end{array}\end{array}$ & $\eta_{i}=\Delta h / \Delta h_{\text {ideal }} / \mathrm{p}_{2}$ & $\Delta h_{\text {ideal }} / U_{2}^{2}$ & $\Delta h_{\text {ideal }} / N_{c o r}^{2}$ \\
\hline $\begin{array}{l}\text { Isentropic efficiency } \\
\text { Polytropic efficiency(perfect } \\
\text { gas) }\end{array}$ & $\eta_{p}=\frac{\gamma}{\gamma-1} \cdot \frac{\ln (T R)}{\ln (P R)}$ & - & - \\
\hline
\end{tabular}


Del-H / Nc^2 and Del-Hi / Nc^2 vs. Wc/Nc (Normalized)

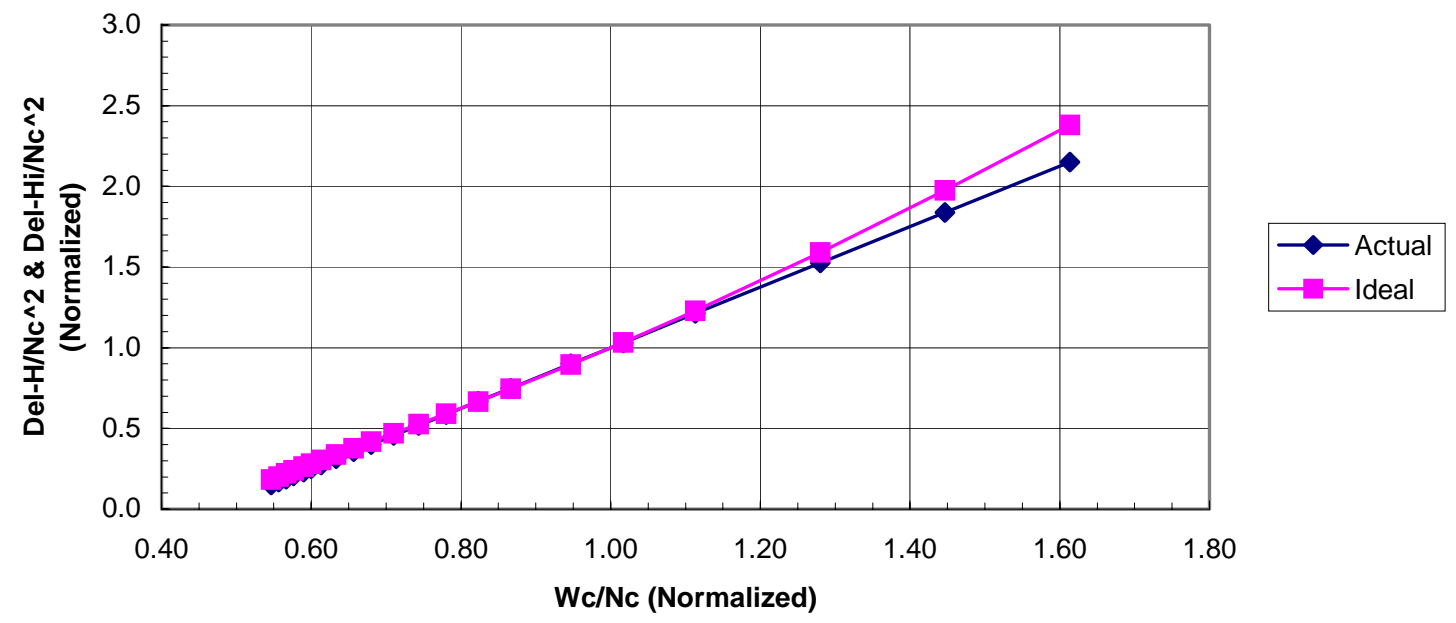

Figure 4.20 Estimated actual and ideal enthalpy rise coefficient versus flow coefficient of a four-stage axial turbine

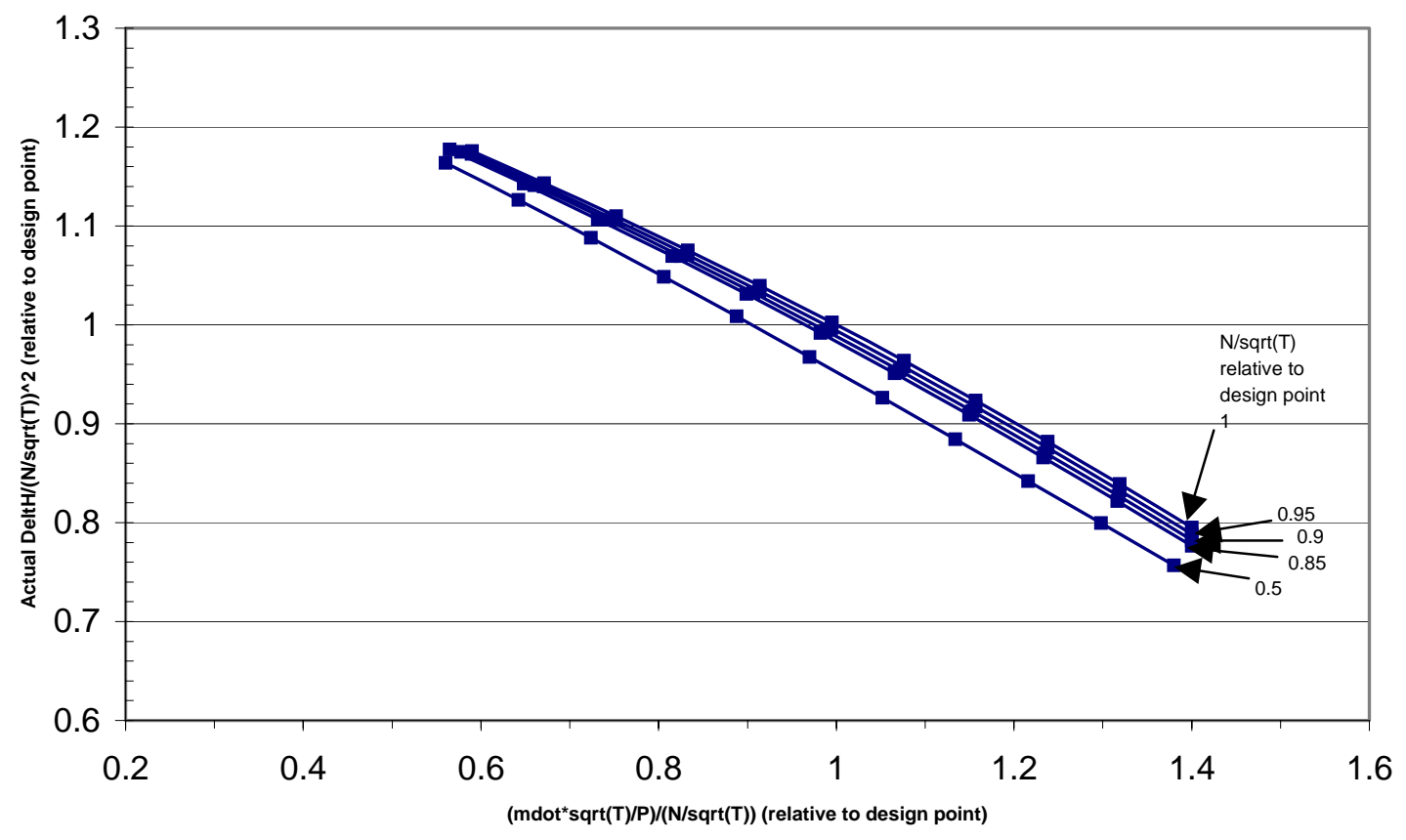

Figure 4.21 Estimated actual Enthalpy Rise coefficient versus corrected flow coefficient of a five-stage centrifugal compressor 


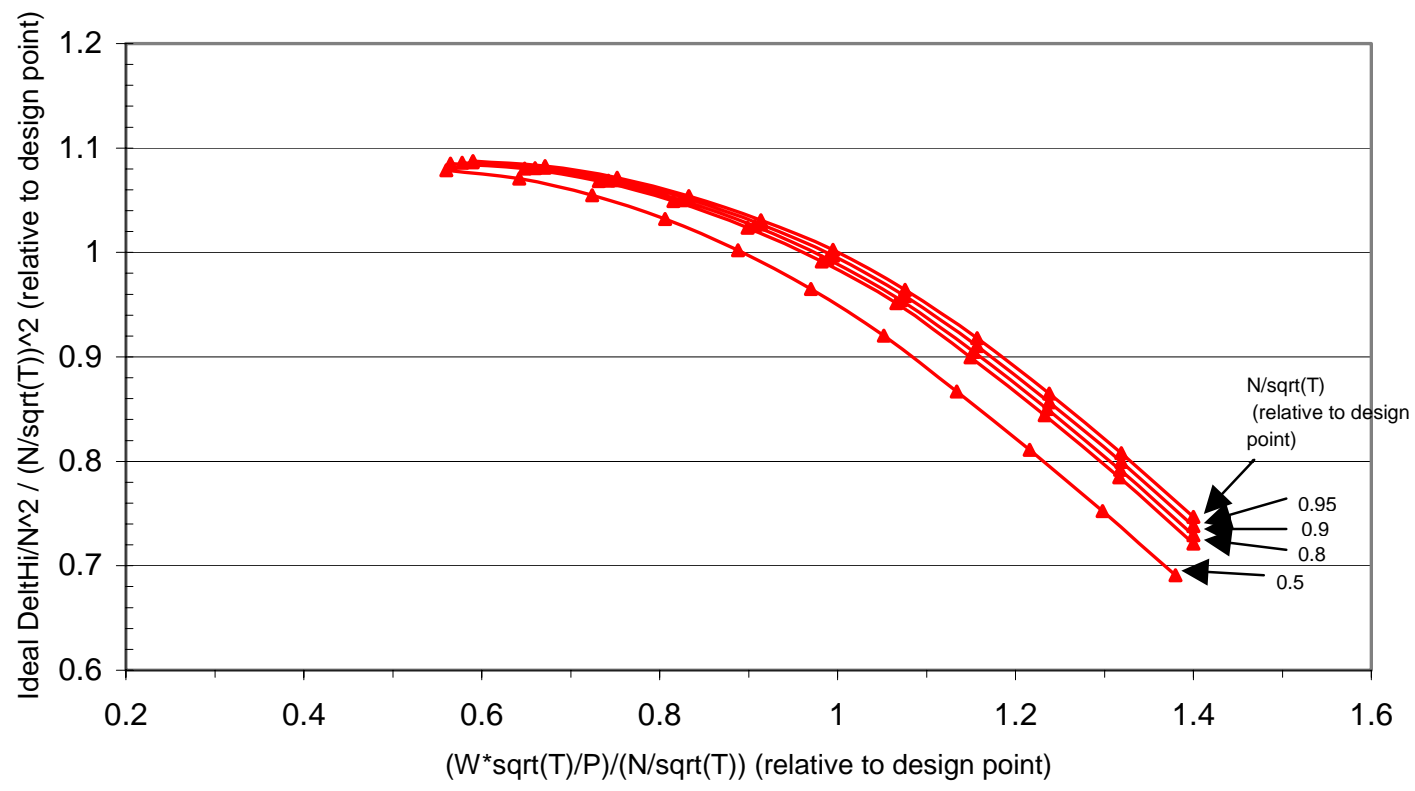

Figure 4.22 Estimated ideal enthalpy rise coefficient versus corrected flow coefficient of a five-stage centrifugal compressor

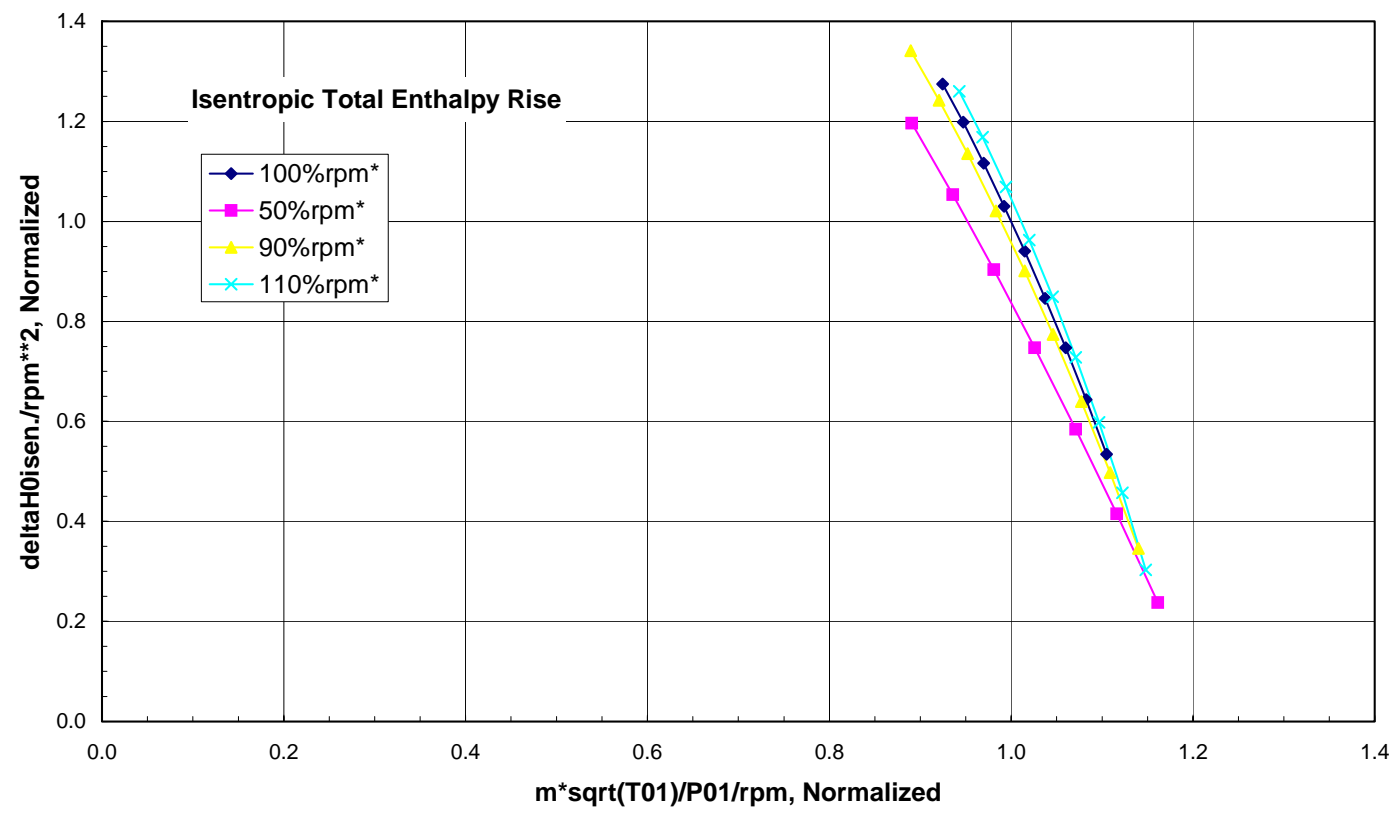

Figure 4.23 Estimated ideal enthalpy rise coefficient versus flow coefficient of an $8+1$ axicentrifugal compressor 


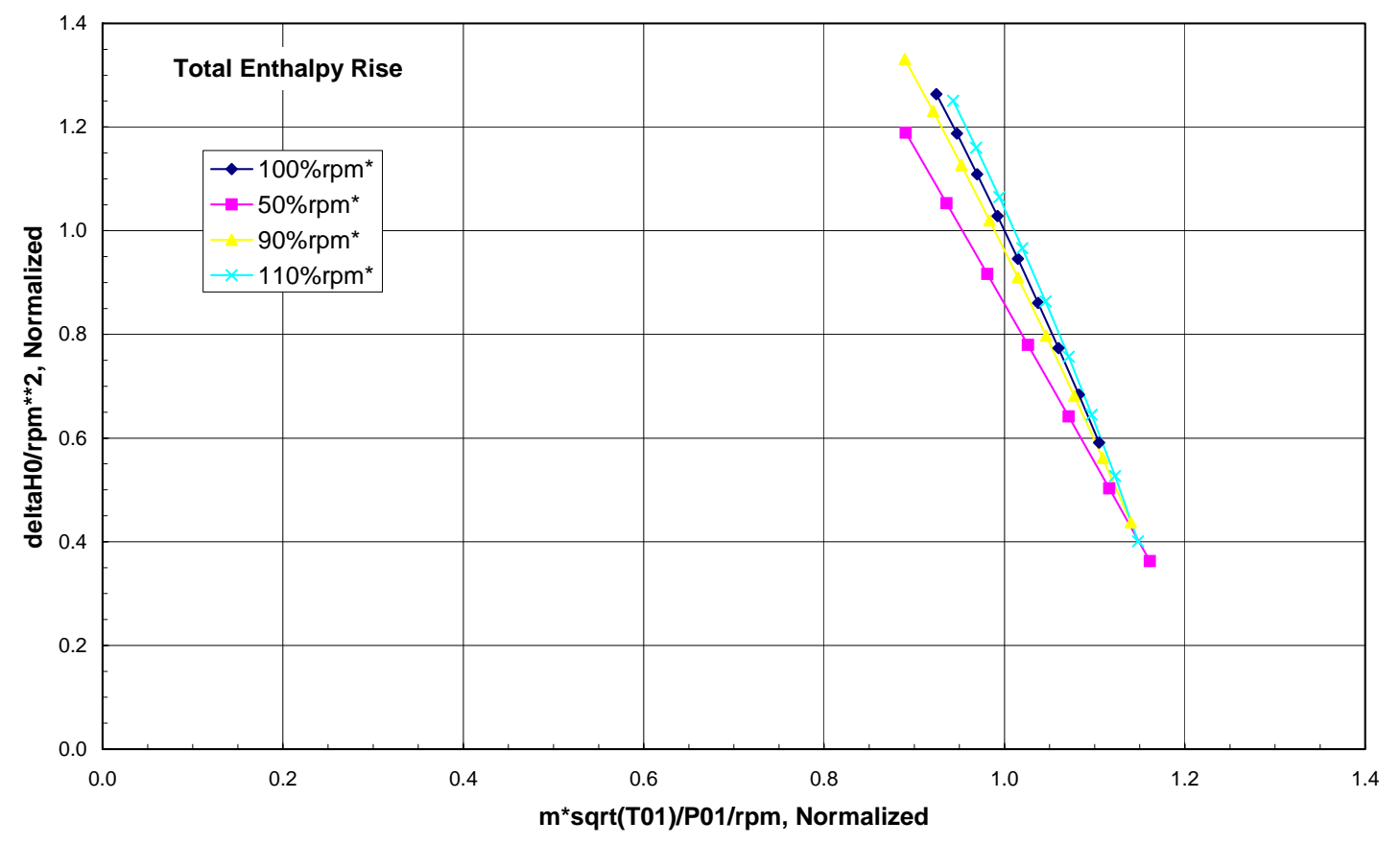

Figure 4.24 Estimate actual enthalpy rise coefficient versus flow coefficient of an $8+1$ axicentrifugal compressor

\subsubsection{Shaft And Generator Model}

In the power conversion system of the MPBR, there are three shafts -- the HP turbine and LP turbine drive two compressors respectively and the power turbine provides the mechanical power to the synchronous generator for electric production. For the shafts on which the HP turbine and LP turbine are mounted, the shaft speeds are determined by the net torque exerted on it:

$$
\frac{d N}{d t}=\frac{P_{t}-P_{C}-P_{\text {loss }}}{\left(I_{t}+I_{C}\right) \omega}
$$

where $\mathrm{N}$ is the shaft rotational speed, $\mathrm{P}_{t}$ is the turbine power, $\mathrm{P}_{\mathrm{c}}$ is the compressor power and $\mathrm{P}_{\text {loss }}$ is the power loss caused by mechanical friction and windage. $\mathrm{I}_{t}$ is the turbine momentum inertia and $\mathrm{I}_{\mathrm{c}}$ is the compressor momentum inertia. For the HP turbine, LP turbine and each compressor the inertia is estimated to be 100,100 and $100 \mathrm{~kg} \mathrm{~m}^{2}$, respectively. The mechanical losses on the shaft are estimated to be $1 \%$ of the turbine power.

In normal operating and normal load transients, the generator is synchronized with an infinite electric grid, which is characterized by a constant voltage and frequency. The generator is kept in synchronization with the grid by the generator power angle. The generator power angle works essentially as an integral control on the turbine-generator 
speed through the electric load[28]. However, in this thesis, we still use the torque balance equation for the turbine-generator shaft for simplification:

$$
\frac{d N}{d t}=\frac{P_{t}-P_{e}}{\left(I_{t}+I_{g}\right) \omega}
$$

where $P_{t}$ is the power of the power turbine, $P_{e}$ is the electric load, $I_{t}$ is the total inertia of the power turbine and $\mathrm{I}_{\mathrm{g}}$ is generator inertia while $\mathrm{N}$ is the shaft rotational speed. For the power turbine and generator the inertia is 300 and $300 \mathrm{~kg} \mathrm{~m}^{2}$, respectively.

\subsubsection{Valve Model}

There are three valves used in the power conversion system - a bypass valve between the outlet of the HP compressor and the inlet of the precooler, a valve for helium leaving the system to the inventory control vessel and a valve for helium feeding back from the inventory control vessel to the system. The flow rate varies with the pressure ratio across the valve so long as valve downstream pressure is higher or equal to the critical pressure. When the valve downstream pressure is lower than the critical pressure, the flow rate stays constant, and this flow is called choked flow.

The flow rate through a valve is limited by choked flow and can be calculated by the following equation based on single-phase flow[11].

$$
\dot{m}=A \rho_{1} \sqrt{2 c_{p} T_{1}\left[\left(\frac{p_{2}}{p_{1}}\right)^{2 / \gamma}-\left(\frac{p_{2}}{p_{1}}\right)^{(\gamma+1) / \gamma}\right]}
$$

The critical pressure can be determined by:

$$
\left(\frac{p_{2}}{p_{1}}\right)_{c r}=\left(\frac{2}{\gamma+1}\right)^{\frac{\gamma}{\gamma-1}}
$$

where $\gamma$ is $C_{p} / C_{V}$ : for helium, the critical pressure ratio is $0.487, p_{1}$ is pressure upstream of the valve $(\mathrm{Pa}), \mathrm{T}_{1}$ is temperature upstream of the valve $(\mathrm{K}), \rho_{1}$ is density upstream, $\left(\mathrm{kg} / \mathrm{m}^{3}\right), \mathrm{A}$ is valve area $\left(\mathrm{m}^{2}\right), \mathrm{p}_{2}$ is pressure downstream of the valve $(\mathrm{Pa})$ and $\dot{m}$ is mass flowrate $(\mathrm{kg} / \mathrm{s})$.

The diameter of all three valves is assumed to be1 1 inches $(27.9 \mathrm{~cm})$. The valve opening delay when responding to the actuator action is ignored.

\subsubsection{Pipe Model}

For a pipe with length $L$ and cross section area A, as shown Figure 4.26, the momentum equation is as following: 


$$
\frac{d \dot{m}}{d t}=\frac{A\left(p_{1}-p_{2}\right)}{L}-f \frac{\dot{m}^{2}}{2 \rho D_{e} A}-\frac{1}{A L}\left(\frac{\dot{m}_{1}^{2}}{\rho_{1}}-\frac{\dot{m}_{2}^{2}}{\rho_{2}}\right)
$$

where $\dot{m}$ is the mass flowrate in the pipe, $\mathrm{A}$ is cross section area, $\mathrm{L}$ is pipe length, $\mathrm{p}_{1}$ is inlet pressure and $\mathrm{p}_{2}$ is outlet pressure, $f$ is friction factor, $\rho$ is the density, De is hydraulic diameter, $\dot{m}_{1}$ is inlet mass flowrate and $\dot{m}_{2}$ is outlet flowrate, $\rho_{1}$ is inlet density and $\rho_{2}$ is outlet density.

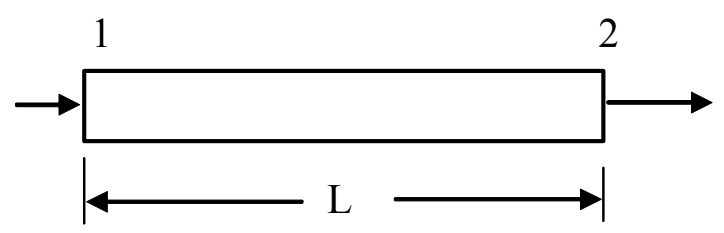

Figure 4.25 The sketch of a pipe

The pipe model is defined by the following simplified equation:

$$
\frac{d \dot{m}}{d t}=K \cdot\left(P_{1}-P_{2}-K_{f r} \dot{m}^{2}\right) \cdot A / L
$$

where $\mathrm{K}_{\mathrm{fr}}$ is a friction coefficient and $\mathrm{K}$ is a coefficient, taken as 0.1 . The value of $\mathrm{K}$ is chosen for numerical stability reasons. It will not influence the dynamics of the system but enhance numerical stability. In the dynamic model, the pipe diameter is taken as $0.5 \mathrm{~m}$. The total length is taken as $20 \mathrm{~m}$.

\subsubsection{PI Controller}

PID (Proportional-plus-Integral-plus-Derivative) controllers and modified PID controllers are widely used in the field of process control systems. It is well known that they provide satisfactory control, though they may not provide the optimal control in some situations. In this work, PI controllers will be used as automatic controllers. The control action of a PI controller is defined by

$u(t)=K_{p} e(t)+\frac{K_{p}}{T_{i}} \int_{0}^{t} e(t) d t$

where $\mathrm{K}_{\mathrm{p}}$ is the proportional gain, and $\mathrm{T}_{\mathrm{i}}$ is the integral time, $\mathrm{e}(\mathrm{t})$ is error signal and $\mathrm{u}(\mathrm{t})$ is the output signal to an actuator. Both $\mathrm{Kp}$ and $\mathrm{T}_{\mathrm{i}}$ are tuning parameters.

\subsubsection{Integration Of Component Sub-Models}


The overall model was integrated and programmed using the Advanced Continuous Simulation Language (ACSL), designated MPBRSim. The flowchart is shown in Figure 4.26. For the closed cycle, the pressure is the most import parameter in iteration to get convergent results. The ACSL implicit integration operator, IMPLC, is used to carry out the iteration. The fourth order Runge-Kutta algorithm is used to solve the differential equations. Two types of the compressor map are used - centrifugal and axial. As the speed line in the axial compressor map is much steeper than that of the centrifugal compressor map, the time step size is much smaller when we use an axial compressor map. For a centrifugal compressor map, the time step size is $0.02 \mathrm{~s}$ while it is $1 \times 10^{-5} \mathrm{~s}$ for an axial compressor map.

\subsection{Summary}

This section presents the numerical methods for the steady state model and the dynamic model. The component losses affecting plant performance are described in detail. For the dynamic model, the component sub-models are described individually and their integration and the numerical solution method are provided. Verification for some component submodels is performed. 


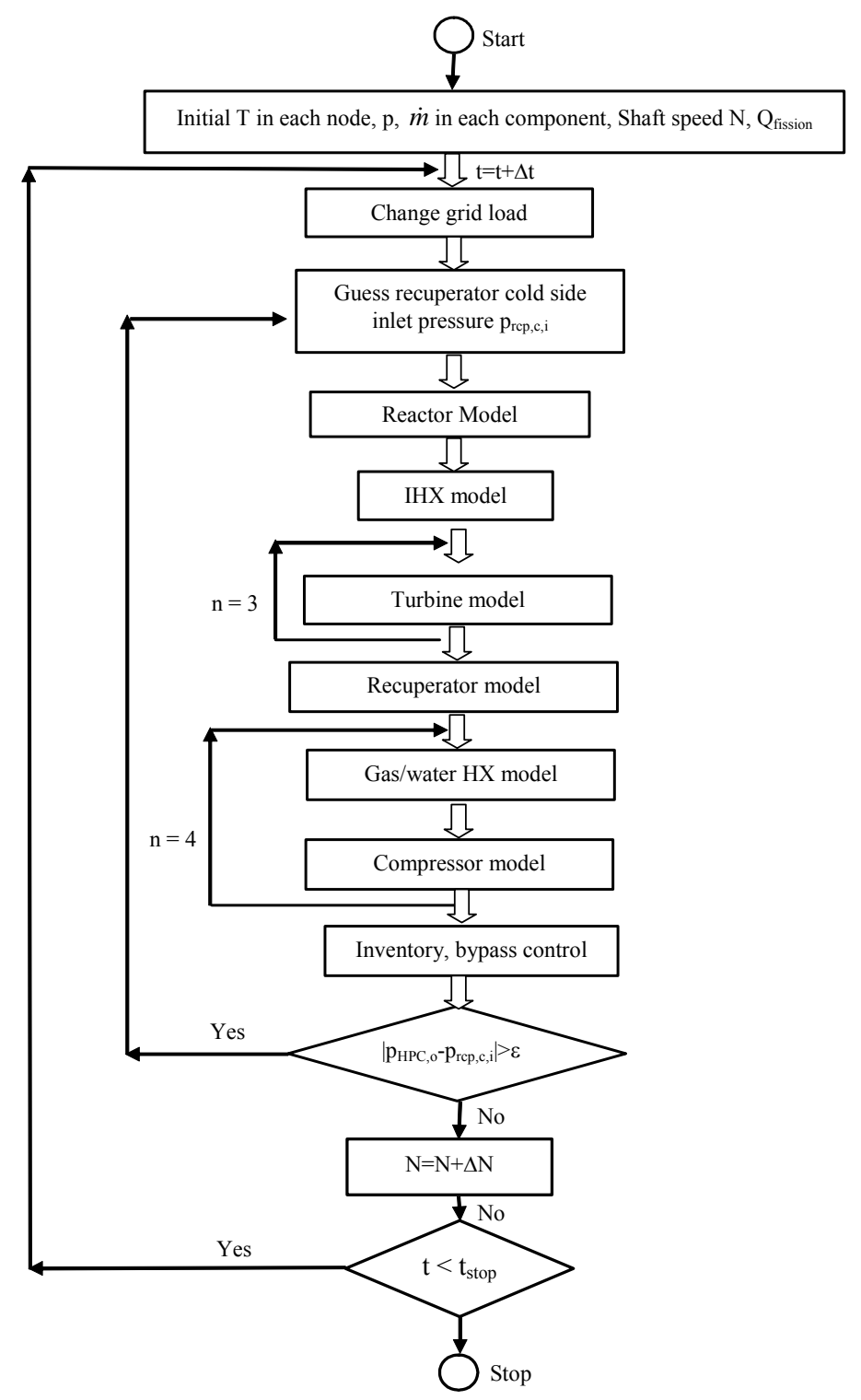

\section{References: Section 4}

Figure 4.26 Flowchart of the dynamic model

[1] Xinglong Yan, "Dynamic analysis and control system design for an advanced nuclear gas turbine power plant", MIT PhD thesis, 1990.

[2] E.C. Verkerk, "Dynamics of the pebble-bed nuclear reactor in the direct Brayton cycle", $\mathrm{PhD}$ theis, Delft University of Technology, Netherlands, 2000.

[3]J.F. Kikstra, A.H.M.Verkooijen, "Dynamic modeling of a cogenerating nuclear gas turbine plant", Journal of Engineering for Gas Turbines and Power, Vol.124, July, 2002. 
[4] G.P. Greyvenstein, P. G. Rousseau, "Design of a physical model of the PBMR with the aid of Flownet", Proceedings of the Conference on High Temperature Reactors, Petten, Netherlands, April 22-24, 2002.

[5] C. Rodriguez, J. Zgliczynski, D. Pfremmer, “GT-MHR Operations and control”, IAEA technical committee meeting on "Development status of Modular High Temperature and their future role," Nov. 28-30, 1994, ECN, Petten, Netherlands.

[6] H. Cohen, G. F. C. Rogers, H. I. H. Saravanamuttoo, "Gas turbine theory", Third edition, Longman Scientific \& Technical, 1987.

[7] D. G. Wilson, T. Korakianitis, "The design of high-efficiency turbomachinery and gas turbines", MIT press, 1998.

[8] P. P. Walsh, P. Fletcher, "Gas turbine performance”, Blackwell Science, 1998.

[9] V.F. Golovko, I.V. Dmitrieva, N.G. Kodochigov, N.G. Kuzavkov, A. Shenoy, "Features of adapting gas turbine cycle and heat exchangers for HTGRs", IAEA TCM on "Gas

Turbine Power Conversion Systems for Modular HTGRs”, Nov. 14-16 2000, Palo Alto, California.

[10] Personnel communication with Dr. H. C. No, August, 2001.

[11] N. E. Todreas, M. S. Kazimi, "Nuclear system I: Thermal hydraulic fundamentals", Taylor \& Francis.

[12] W. M. Rohsenow, et. al., "Handbook of heat transfer", pp.5.30-5.32, Third edition, McGram Hill, 1998.

[13] Aegis Simulation, Inc. "Advanced Continuous Simulation Language(ACSL), Reference Manual", Sept. 1999.

[14] K. E. Brenan, S. L. Campbell, L. R. Petzold, "Numerical solution of initial value problems in differential algebraic equations", North-Holland, 1989.

[15] "Waermeuebergang im Kugelhaufen," KTA 3102.1 KTA, June 1978.

[16] G. Breitbach, H. Barthels, "The Radiant Heat Transfer in the High Temperature Reactor Core After Failure of the After Heat Removal System," Nuclear Technology, Vol. 49, August 1980.

[17] D. Saphier, "HTGR Transient Analysis with the DSNP Simulation Language," RASG111-84, Soreq Nuclear Research Center, Israel, December 1984.

[18] M.G. Savage, “A One-Dimensional Modeling of Radial Heat Removal During Depressurized Heatup Transients in Modular Pebble-Bed and Prismatic High Temperature Gas-Cooled Reactors," ORNL/TM-9215, Oak Ridge National Laboratory, July 1984. [19] R. E. Nightingale, et. al., "Nuclear Graphite", Academic Press, New York, 1962. [20] H. Ethrington, et. al.,"Nuclear Engineering Handbook," McGraw-Hill, New York, 1968.

[21] General Atomic Company, "The thermodynamic and transport properties of Helium". October, 1975.

[22] Allan F. Henry, "Nuclear Reactor Analysis", MIT Press, 1997.

[23] Personal communication with Dr. Julian Lebenhaft , April 07,2000.

[24] Personal communication with Dr. Eban Mulder, Dec. 19,2002.

[25] Concept-NREC, "Computer program for the prediction of thermal transients in compact heat exchangers", Volume I, Aug. 25, 1969.

[26] S.J. Ball, "Initial Simulation and Control System Studies of the MIUS Coal-Fired

Turbine Experiment", ORNL/HUD/MIUS-42, February, 1978.

[27] M-Tech Industrial, P.O.Box 19855, Potchefstroom, South Africa. 
[28] A. E. Fitzgerald,Charles Kingsley, Jr., and Alexander Kusko, "Electric machinery", New York, McGraw Hill, 1971. 


\section{Results: Control system design}

\subsection{Introduction}

In the MPBR design, we use an indirect cycle, in which an IHX is used to separate the primary system and the "secondary" system (power conversion unit). In the PCU, a threeshaft arrangement is adopted, as shown in Figure 5.1. The generator is synchronized with the electric grid. Thus the shaft of the turbine-generator rotates at a rotational speed of 3600 rpm. The High-Pressure (HP) turbine and Low-Pressure (LP) turbine drive two compressors, respectively. In the nominal condition, the shaft of the HP turbine and the shaft of the LP turbine both operate with a rotational speed of $8,000 \mathrm{rpm}$ if axial compressors are adopted and 5,900 rpm if centrifugal compressors are used. During a load transient, the shaft of the HP turbine and the shaft of the LP turbine are both floated. This arrangement necessitates a control system to ensure the plant system operates smoothly during normal operation while providing safety protection to the plant for anticipated accidents. Because the MPBR design is characterized by a high efficiency system, it requires that the plant should remain at high efficiency at partial load operation. And, during normal transients, the control system is used for meeting the load following requirement as well as for minimizing the thermal stress impact on the plant components.

In the primary system, the control system implements the conventional reactor control, such as regulating the reactor power to match the load requirement, which limiting the core outlet temperature. Startup and shutdown of the reactor are also realized by the reactor control system. In the PCU, the closed cycle provides a unique control potential and availability for regulating electricity output from the system. Bypass mass flow, mass inventory reduction and variation of guide vane angle are potential methods for power conversion system control. We deliberately integrate the control methods in the primary system and those in the PCU to achieve the expected plant transient performance and to minimize the thermal stress impact on the components during transients.

\subsection{Control Strategy}

The MPBR control system must be capable of meeting utility requirements for load following and control band. For the system developed in this thesis the following design requirements have been imposed on the control system:

(1) Normal Power Control Band: $50-100 \%$

(2) Power Reduction During Normal Operation: 10\%/min

(3) Fast Power Reduction During Normal Operation: 10\% step change

(4) Power Increase During Normal Operation: 5\%/min

(5) Load Rejection-Transient: 100\% Instantaneous Grid Separation 


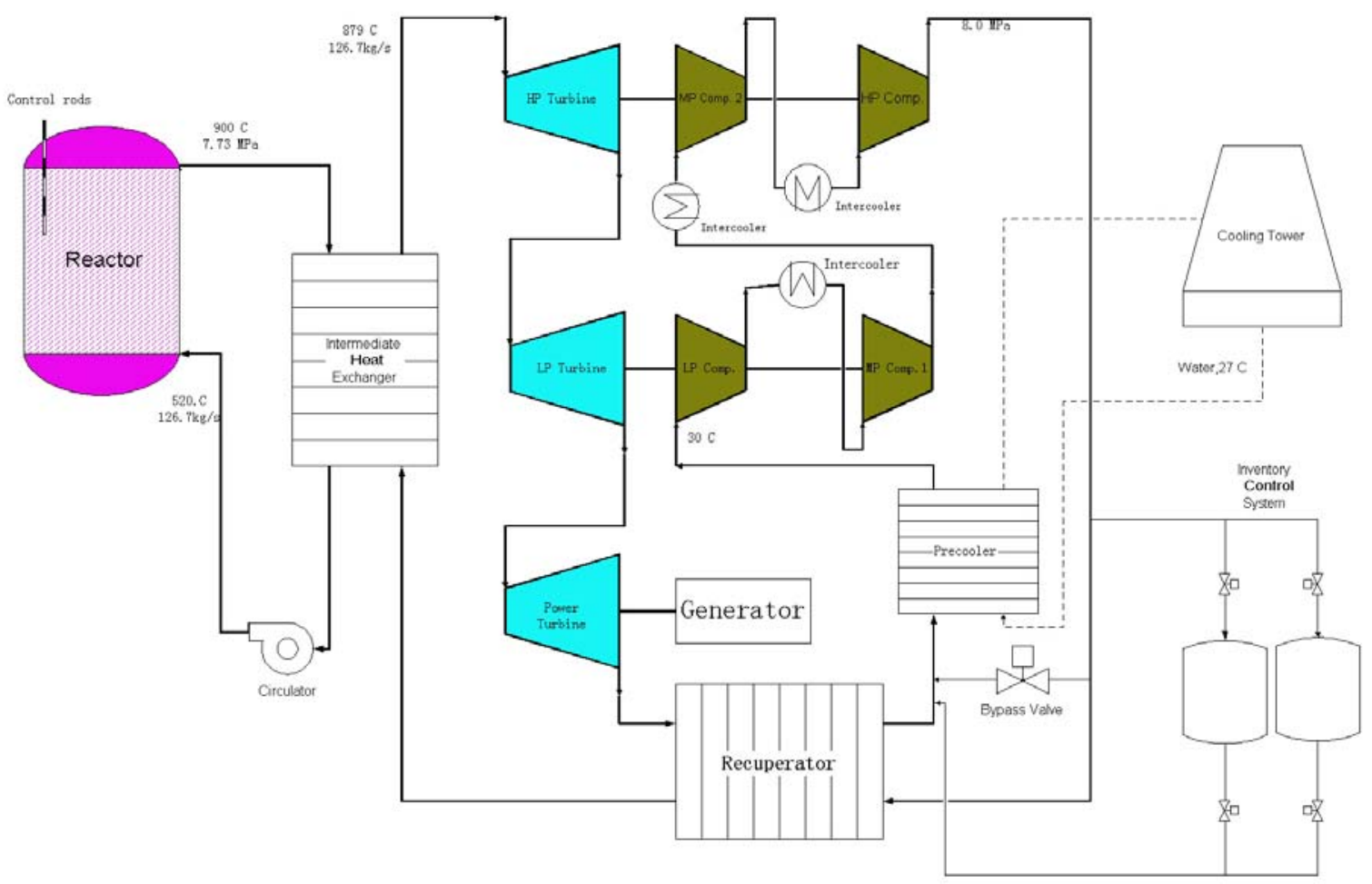

Figure 5.1 Control configuration for the MPBR

During normal operation, the control equipment in the primary system and in the PCU is all in service to ensure that the plant supply the electricity load demanded by the grid with the necessary reliability. The main transients during normal operation are load transients, which occur at start up, shut down and during grid load following. Smooth transition from one load level to another level is a reliability and safety requirement of the plant. The main control goal during a load ramp is avoiding grid separation. One of the control tasks is to maintain the turbine-generator shaft speed $60 \pm 0.5 \mathrm{~Hz}$ during normal operations when the generator is locked to the grid [1]. Grid separation is mainly caused by the mismatch of the frequency of the electricity generated and the frequency of the grid. The grid frequency is the synchronized frequency of all the generators connected to the grid. In terms of this plant, the grid can be treated as an infinite bus. When the power produced by the power turbine differs from the load demand, the turbine-generator shaft accelerates or decelerates. When the frequency difference is over a limit, grid separation occurs. During startup to nominal operation and during planned shutdown, there should be grid separation. The startup and planned shutdown procedures will be investigated in the future.

Tripping of plant always causes grid separation. Thus it causes a 100\% load rejection from full load to no load in a very short time (a fraction of second [2]). In this case, the overspeed of the turbine-generator has to be controlled within the generator mechanical design limit. The over-speed limitation for the generator depends on its design; the value is taken as $120 \%$ here[3]. 
Due to materials limitations and the high efficiency goal, the core outlet temperature needs to be maintained at $900^{\circ} \mathrm{C}$ in partial load operation. Given the concern of the thermocyclic failure of the components, minimizing the thermal stress impact on the component is another main consideration for control system design.

In summary, the control objectives are as follows:

(1) During normal operation, maintaining the turbine-generator shaft speed $3600 \pm 30$ rpm;

(2) Maintaining high efficiency during partial load operation;

(3) Maintaining the core outlet temperature of $900^{\circ} \mathrm{C}$ during partial load operation;

(4) Minimizing the thermal stress impact on the components during load transients;

(5) For $100 \%$ load rejection, maintaining the turbine-generator shaft speed within $120 \%$.

\subsection{Control Methods}

For the indirect cycle, typically, there are two potential control methods in the primary system and three in the PCU. In the primary system, there are control rod movement control and circulator speed control. Bypass valve control, inventory control and turbine vane angle control are the potential control methods in the PCU. The control methods and their functions are described in the following.

\section{- Bypass Valve Control}

Bypass valves divert the helium flow from the hot side of the IHX (heat source in terms of the PCU) and all the turbines. When the bypass valves open, the flow admitted to the hot side of the IHX and all the turbines is less. Meanwhile, the pressure ratios of the turbines decrease. As a result, the shaft power delivered to the generator is reduced by the combined effect of the lowered turbine flow and lowered turbine pressure ratio.

Bypass valve control is used to provide for rapid power manipulation, especially for power reductions. For example, for a $10 \%$ of grid load step reduction, the bypass valve opens to make the power produced by the power turbine follow load requirement and prevent a grid separation. It is also used to prevent turbine shaft overspeed while the generator is not locked to the grid.

The bypass valve can quickly reduce the system electric output. But, as the bypass valve opens, the working fluid volume flow decreases. The turbine work point shifts from the design point, and reduces the turbine efficiency. This is the reason that the plant efficiency is reduced when bypass valve control is used. This is corrected by combining the bypass valve control and inventory control as explained below. As the bypass valve opens, the running point of the turbines shift from the design point, as a result, the outlet temperature of the power turbine increases. Thus, there is a thermal shock on the hot inlet side of the recuperator and the cold inlet side of the IHX if the bypass valve opens. Inventory control is used to minimize this increase of the flow temperature in the hot inlet side of the recuperator 
and the cold inlet side of the IHX. The heat load increases significantly in the precooler as the bypass valve used.

\section{- Helium Inventory System}

A helium inventory system normally consists of a series of helium storage vessels, each one with two valves connecting to the high pressure side and the low pressure side of the PCU respectively. The number of helium storage vessels will be determined in the future. As the grid load is reduced, helium will be withdrawn from the high pressure side of the PCU into the vessels. However, if the grid load is to be raised, helium in the vessels is fed back into the secondary system at the low pressure side to increase its inventory. The inventory variation in the closed cycle of the PCU causes a helium gas density change. Lowering the inventory of the PCU results in a reduction of pressure thus the density of working fluid as the temperature remains constant. If the temperatures of the PCU are kept constant, the sonic speed will not change. And blading and flow passage geometric design fix the local Mach numbers. Thus the local velocities are constant throughout the cycle of the PCU. Inventory lowering reduces the power turbine output and electric output by reducing the massflow through the PCU and vice versa. The turbomachines still operate near their design points, since the pressures at every point of the cycle vary in roughly the same ratio while the temperatures and flow velocity remain unaltered. Therefore, the plant efficiency remains high.

The drawback of an inventory control system is that it is not capable of rapid power manipulation. This is because of the relatively large time constants characterizing the helium transfer between the storage vessels and PCU. For this reason, an inventory control system is used in parallel with the other control method, i.e. the bypass valve control.

In order to eliminate the large pressure differential between the primary side and the secondary side when inventory control is used for partial load operation, inventory control is also considered to be used for the primary system.

\section{- Control Rod Movement}

The heat generated in the reactor core is the only heat source for input to the turbines. As the grid electric load varies, to match it, the mechanical power delivered to the generator from the turbine must be varied. Therefore, the reactor power must be quickly controlled to provide the required thermal power input to the PCU. Adjusting the control rod position in the reflector can change the neutron flux level, thus changing the reactor power. Furthermore, the rods can shut down the reactor and keep it subcritical at any operating condition. Also, the reactivity induced by the control rods movement should compensate for the reactivity caused by the fission products (i.e. xenon) and the fuel and moderator temperature coefficient of reactivity during reactor power variation.

The control shutdown system consists of two reactivity subsystems. One is the reactivity control system and the other is the small-sphere shutdown system. The reactivity control system consists of two sets of control rods located in the side reflector; one set of control rods can be moved freely for regulating power control while the other set is for shutdown of 
the reactor. If hot shutdown is required, the control rods drop by gravity into the core. For long-term, cold reactor shutdown, the small-sphere shutdown system is used. The poisoncontaining small spheres are released into the columns in the side reflector.

Although control rod movement provides the capability of regulating the reactor power, it probably is not needed for normal operating transients due to the high negative temperature coefficient of reactivity of the MPBR design. This is a significant advantage of the pebble bed reactor system.

\section{- Circulator Speed Variation}

The circulator circulates the helium through the primary system. The mass flowrate is proportional to the circulator speed as the inventory in the primary system remains unaltered. If the circulator speed is adjusted, the mass flowrate is changed correspondingly. If the reactor power decreases, the core outlet temperature decreases under the condition that the core inlet temperature and mass flowrate remain unaltered. Then the inlet temperature of the turbines in the PCU decreases. This results in a reduction of the turbine efficiency. To maintain high temperature to the turbine, circulator speed variation is used to maintain the core outlet temperature constant.

\section{- Vane Angle Control}

Both for gas turbines and compressors, the map is unique for a fixed value of inlet flow angle. Changing the inlet flow angle will modify the map in certain key operating ranges. A suite of maps can be plotted with the inlet flow angle as an independent variable. VIGVs (Variable inlet guide vanes) for compressors and variable area NGVs (nozzle guide vanes) for turbines provide working line control to ensure the operating point without crossing the surge line $[4,5]$.

VIGVs are mainly employed to allow a compressor to have an acceptable low speed surge line. Variable area NGVs are occasionally used on LP or power turbines for recuperated cycles to maintain high turbine outlet temperature, and hence heat recovery, at partial load[5]. Compared with compressor VIGVs, the turbine NGVs work in a far higher temperature environment. As a result, the operating mechanism for control turbine NGVs is expensive and complex. It is not practical to use the NGVs for the HP turbine due to the high temperature. In this work, vane control is not included.

\subsection{Configuration Of Control System}

The control system configuration for the current design is shown in Figure 5.1. The bypass valve is located between the outlet HP compressor and inlet precooler, at which point the temperatures are low, allowing conventional industrial valves to be used. Currently, the helium inventory system consists of two storage tanks, which are connected via two pairs of inventory control valves with the PCU. The volume of the tanks will be determined in further design work. One pair of inventory control valves connect with the inlet precooler 
while another pair connect with the outlet HP compressor. This configuration requires no compressor between the vessels and the PCU.

In the situation of total load rejection - grid separation, the concern is the overspeed of the turbine-generator shaft. It is necessary to generate an alarm signal and completely stop the helium circulation through the power turbine right after the accident. Due to the high temperature, it is difficult to use a bypass valve around the power turbine to divert the helium flow. The solution to prevent overspeed of the generator in the condition of grid separation is quickly connecting the generator to a resisting load source, such as an air or water cooled resistor heater as a turbine-generator brake.

As shown in Figure 5.1, the circulator is installed at the location which connects the outlet of the hot side of the IHX and the core inlet. Thus this takes advantage of the low temperature of the primary cycle. The circulator is driven by a variable speed electric motor, its speed is adjusted continuously. As a result, variable mass flowrate of the primary system is achieved.

\subsection{Automatic Control System}

To achieve the power control goal, individual control subsystems must coordinate with each other based on the control strategy. In the MBPR design, the control system is facilitated by a set of PI controllers using feedback loops, as shown in Figure 5.2. The controllers control several independent variables to perform the control functions. These are:

(a) Turbine-generator shaft speed;

(b) Helium mass inventory in the inventory vessel or the bypass valve position;

(c) Core outlet temperature;

(d) Mass flowrate of the primary system.

There are several controllers: inventory valve controller, bypass valve controller, circulator speed controller and control rod controller. The controllers send their signals to the corresponding actuators to operate of the control subsystems. Taking the bypass valve controller as an example, it receives the regulating command signal, which calls for the turbine-generator shaft speed which the operator demands (e.g. 3,600 rpm), and compares the shaft's actual speed measured by the sensor, and thus generates an error. This error is used by the controller to generate a control signal based on the PI algorithm, then the control signal is sent to the bypass valve actuator to manipulate the opening of the bypass valve. In order to prevent continuously opening and closing of valves caused by small variations of the controlled variables, a small threshold is put on the control signal. This control process is continued to ensure the actual turbine-generator shaft speed matches the desired speed of the regulating demand.

The preliminary design of the control system has been described above. Since the components of the PCU have not all been designed, in the preliminary simulation, $1000 \mathrm{~m}^{3}$ of volume is assumed for the PCU and $500 \mathrm{~m}^{3}$ for the inventory vessels.

Vane angle control is not used in the current design stage. The PI controller tuning parameters are listed in Table 5.1. 


\subsection{Summary}

This section presents the control strategies for the MPBR and the potential control methods on the reactor side and the PCU side. The configuration of the control system is designed and the control algorithm is defined. The transient performance of the plant using the designed control scheme will be shown in the following section.

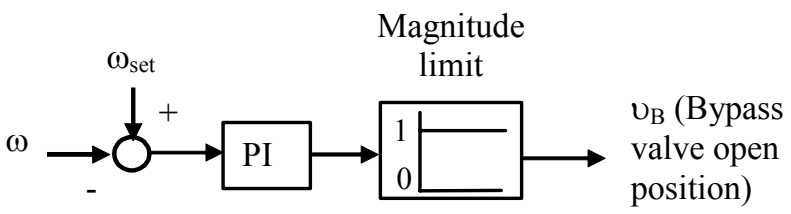

Bypass valve controller

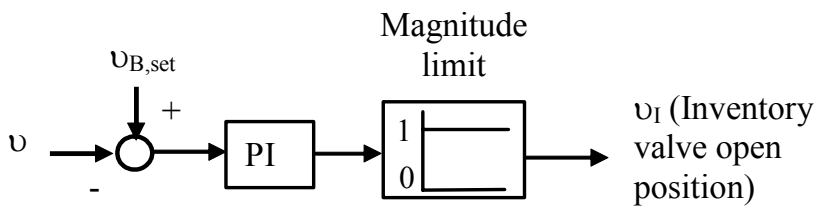

Inventory valve controller

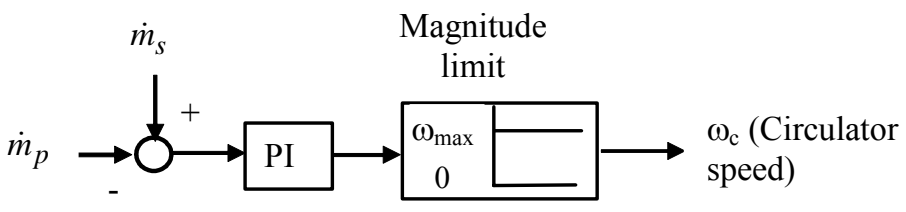

Circulator controller

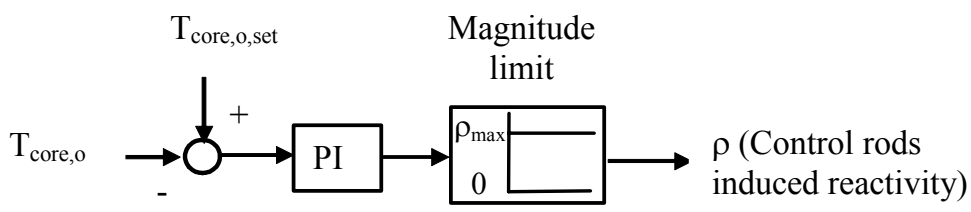

Control rods controller

Figure 5.2 Control feed back loops 
Table 5.1 Parameters of PI controllers

\begin{tabular}{|l|l|l|l|l|}
\hline Controller & $\begin{array}{l}\text { Controlled } \\
\text { variable }\end{array}$ & $\begin{array}{l}\text { Manipulated } \\
\text { variable }\end{array}$ & $\mathrm{K}_{\mathrm{P}}$ & $\mathrm{T}_{\mathrm{i}}$ \\
\hline $\begin{array}{l}\text { Bypass valve } \\
\text { controller }\end{array}$ & $\begin{array}{l}\text { Turbine-generator } \\
\text { shaft speed }\end{array}$ & $\begin{array}{l}\text { Bypass valve open } \\
\text { position }\end{array}$ & $1 \times 10^{-4}$ & $1 \times 10^{-5}$ \\
\hline $\begin{array}{l}\text { Inventory valve } \\
\text { controller }\end{array}$ & $\begin{array}{l}\text { Bypass valve } \\
\text { open position or } \\
\text { inventory of the } \\
\text { PCU }\end{array}$ & $\begin{array}{l}\text { Inventory valve } \\
\text { open position }\end{array}$ & 0.5 & $1 \times 10^{-7}$ \\
\hline $\begin{array}{l}\text { Circulator } \\
\text { controller }\end{array}$ & $\begin{array}{l}\text { Mass flowrate of } \\
\text { the primary } \\
\text { system }\end{array}$ & Circulator speed & $1 \times 10^{-4}$ & $1 \times 10^{-5}$ \\
\hline $\begin{array}{l}\text { Control rods } \\
\text { controller }\end{array}$ & $\begin{array}{l}\text { Core outlet } \\
\text { temperature }\end{array}$ & $\begin{array}{l}\text { Reactivity induced } \\
\text { by control rods }\end{array}$ & $1 \times 10^{-4}$ & $1 \times 10^{-7}$ \\
\hline
\end{tabular}

\section{References: Section 5}

[1] C. Rodriguez, etc, "GT-MHR operations and control", General Atomics Project 7600 Nov. 1994.

[2] KN Pradeep Kumar, A. Tourlidakis, P. Pilidis, "Generation and control of electricity in HTGR helium turbine plant", ICONE10, Arlington, VA, April 14-18,2002.

[3] D. Lomba, D. Thiot, "Generator technology for HTGR power plants", IAEA TCM on high temperature gas cooled reactor technology development, Johannesburg, South Africa, 13-15 Nov. 1996.

[4] Richard T. C. Harman, "Gas turbine engineering applications, cycles and characteristics", John Wiley \& Sons, 1981.

[5] Philip P. Walsh, Paul Fletcher," Gas turbine performance", Blackwell Science, 1998. 


\section{Results: Plant analysis}

\subsection{Introduction}

In Section 4 and Section 5, the steady state model and the dynamic model have been described and the control system configuration has been defined. In this section, cycle parametric analysis is performed using the steady state model. Then the dynamic model will be used to evaluate load step transients and load ramps. The bypass valve control method and the inventory control method will be utilized. The transient calculation will demonstrate the interaction between the reactor core and the PCU. The control method will be provided for grid disconnection. For verifying the PCU part of the dynamic model, the load step transient result under the condition of fixing the IHX hot inlet temperature will be compared with a similar Flownet model.

The cycle design and its salient parameters will be given. Overall, the controllability of the cycle will be confirmed.

\subsection{Steady State Parametric Analysis}

The selection of the cycle parameters and configuration has a pronounced effect on the cycle performance. Calculations are performed to evaluate the effects of the cycle parameters on the cycle efficiency. For simplification, the calculations do not include the RPV cooling passages. With regard to other losses, the station load is assumed as $2.5 \mathrm{MWe}$ and other losses are ignored. The expression of cycle efficiency is as follows:

$\eta_{\text {cycle }}=\frac{W_{\text {gen }}-W_{\text {sl }}-W_{\text {cir }}}{Q_{\text {fission }}} \times 100 \%$

where $\eta_{\text {cycle }}$ is the cycle efficiency, $\mathrm{W}_{\text {gen }}$ is the output of the generator, $\mathrm{W}_{\mathrm{sl}}$ is the station load, $\mathrm{W}_{\text {cir }}$ is the power consumed by the circulator and $\mathrm{Q}_{\text {fission }}$ is the reactor thermal power. The following parameters are included in the analyses: Core outlet temperature, compressor inlet temperature, IHX efficiency, turbine polytropic efficiency, compressor polytropic efficiency, recuperator effectiveness, component pressure losses and diverted mass flowrate from compressors due to leakage or cooling. When we study one parameter, such as core outlet temperature, the other parameters of the cycle are assumed constant as the core outlet temperature varies. The analyses are based on the cycle design as shown in Figure 3.10. Other parameters are listed in Table 6.1.The following gives the effect of each parameter on the cycle efficiency.

From Figure 6.1, a core outlet temperature increase of $50^{\circ} \mathrm{C}$ would result in a gain of cycle efficiency of 1.5 percent point. One thing should be pointed out that the potential materials used as core outlet piping and the IHX primary side structure need to be fully tested if the core outlet temperature rises to $950^{\circ} \mathrm{C}$ because metal test data is unavailable in a radioactive environment at such high temperatures. 
Table 6.1 The component parameters for parametric analysis

\begin{tabular}{|l|c|l|c|}
\hline $\begin{array}{c}\text { Reactor } \\
\text { Fission power } \\
\text { Core pressure drop }\end{array}$ & $\begin{array}{c}250 \mathrm{MWth} \\
2 \%\end{array}$ & $\begin{array}{c}\text { Generator } \\
\text { Efficiency }\end{array}$ & $98 \%$ \\
\hline $\begin{array}{c}\text { IHX } \\
\text { Effectiveness } \\
\text { Pressure drop }\end{array}$ & $\begin{array}{c}95 \% \\
1.77 \% \text { (hot side) } \\
2 \% \text { (cold side) } \\
0.1 \mathrm{MPa}\end{array}$ & $\begin{array}{c}\text { Recuperator } \\
\text { Effectiveness } \\
\text { Pressure drop }\end{array}$ & $\begin{array}{c}95 \% \\
0.8 \% \text { (low press. side) } \\
0.13 \% \text { (high press. Side) }\end{array}$ \\
\hline $\begin{array}{c}\text { Circulator difference } \\
\text { Isentropic efficiency }\end{array}$ & $90 \%$ & $\begin{array}{c}\text { Precooler } \\
\text { Pressure drop }\end{array}$ & $0.8 \%$ (helium side) \\
\hline $\begin{array}{c}\text { Turbines } \\
\text { Polytropic efficiency } \\
\text { Mechanical loss }\end{array}$ & $92 \%$ & $\begin{array}{c}\text { Prtercoolers } \\
\text { Pressure drop }\end{array}$ & $0.8 \%$ \\
\hline $\begin{array}{c}\text { Compressors } \\
\text { Polytropic efficiency } \\
\text { Inlet temperature }\end{array}$ & $90 \%$ & $\begin{array}{l}\text { HP compressor } \\
\text { outlet pressure }\end{array}$ & $8 \mathrm{MPa}$ \\
\hline $\begin{array}{l}\text { Motor for driving } \\
\text { circulotor } \\
\text { Efficiency }\end{array}$ & $30{ }^{\circ} \mathrm{C}$ & & \\
\hline
\end{tabular}

\section{- Reactor Core Outlet Temperature}

A gas turbine can produce more work as the turbine inlet temperature increases with a constant pressure ratio. In an indirect cycle, two factors, the core outlet temperature and IHX effectiveness, govern the turbine inlet temperature. Figure 6.1 shows the cycle efficiency as a function of cycle pressure ratio for three core outlet temperatures: $850^{\circ} \mathrm{C}, 900^{\circ} \mathrm{C}$ and $950^{\circ} \mathrm{C}$. The pressure ratio is defined as the ratio of the lowest pressure to the highest pressure of the secondary cycle. As can be seen, for a specific core outlet temperature, there is an optimum cycle pressure ratio which gives the highest cycle efficiency. For example, with a core outlet temperature of $850^{\circ} \mathrm{C}$, the highest cycle efficiency is about $48.2 \%$ and the pressure ratio is about 2.6. In this case, the core inlet temperature is $525^{\circ} \mathrm{C}$. As mentioned before, the IHX primary outlet temperature is limited to $427^{\circ} \mathrm{C}$ in conformity with ASME code Section III class I. Thus active cooling must be considered for any design . 


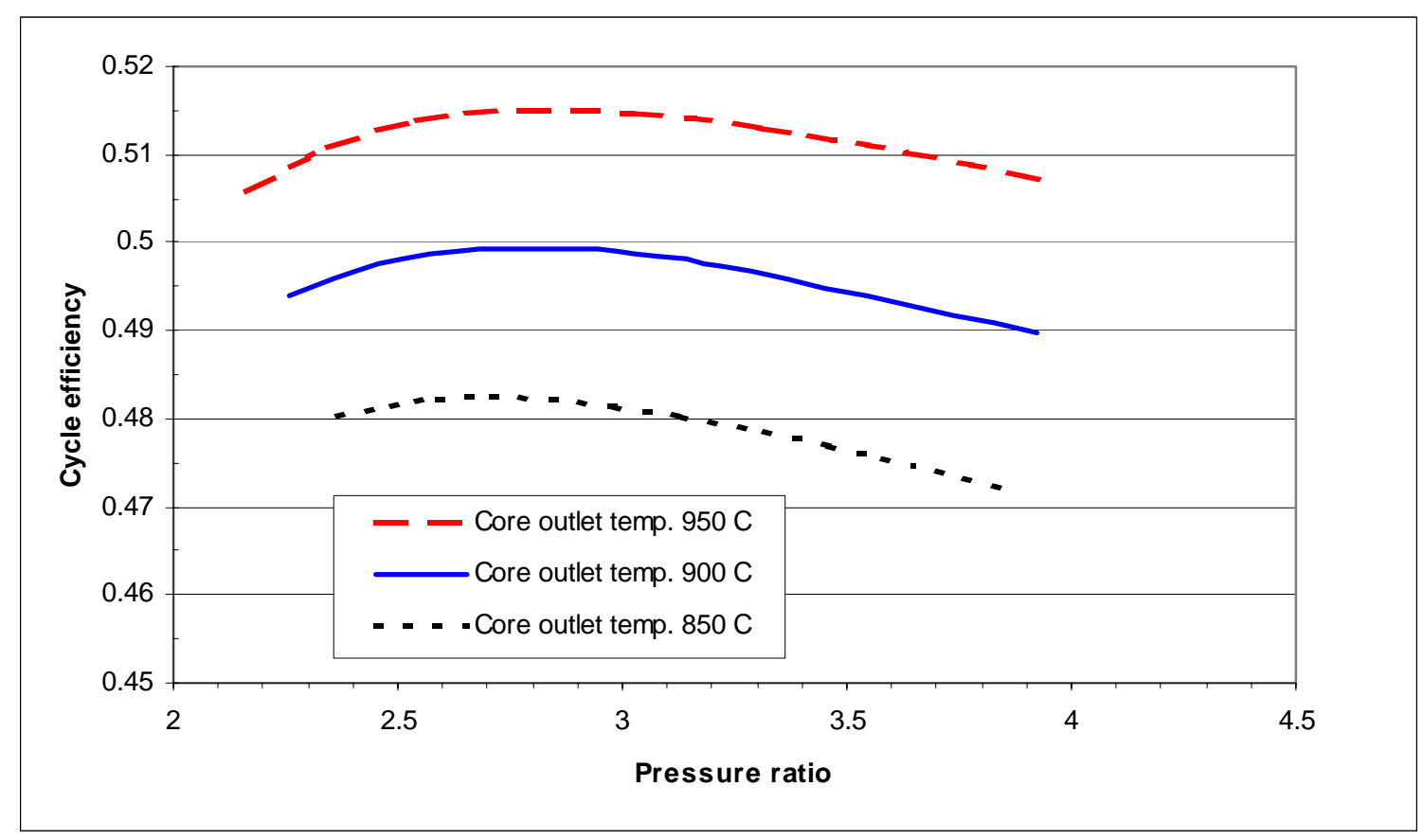

Figure 6.1 Cycle efficiency versus the pressure ratio as a function of core outlet temperature

\section{- Compressor Inlet Temperature}

In the cycle design, the compressors are driven by the turbines. If the consumed power of the compressor decreases, it would increase the net output power of the cycle, thus increase the cycle efficiency. For a compressor, decreasing its inlet temperature causes the consumed power to decrease when maintaining the same pressure ratio. Figure 6.2 shows the cycle efficiency and pressure ratio versus the compressor inlet temperature. From this figure, the compressor inlet temperature has a significant effect on the cycle efficiency. Decreasing the compressor inlet temperature by $1^{\circ} \mathrm{C}$ can achieve an cycle efficiency increase by a value of 0.13 percentage point. That means that an $8^{\circ} \mathrm{C}$ the compressor inlet temperature reduction causes a cycle efficiency gain of around 1 percentage point.

In the cycle design, the precooler and intercooler are usually used to cool the working fluid before it enters the compressors. In the MPBR, the working fluid is helium. In consideration of the size of the precooler and intercooler, the precooler and intercooler are helium/water type heat exchangers. MPBR is supposed to be built not only offshore of rivers, lakes and the sea but also in the desert areas lacking water. Therefore, in the MPBR, the cooling water of the precooler and intercooler is supplied by a cooling tower. The outlet water temperature of the cooling tower depends on the local weather where the plant is located. In the MPBR system design, the outlet water temperature of the cooling tower is assumed to be $27^{\circ} \mathrm{C}$. Comparing with other similar system designs such as the GT-MHR, which the water temperature is assumed $22^{\circ} \mathrm{C}, 27^{\circ} \mathrm{C}$ is conservative. 


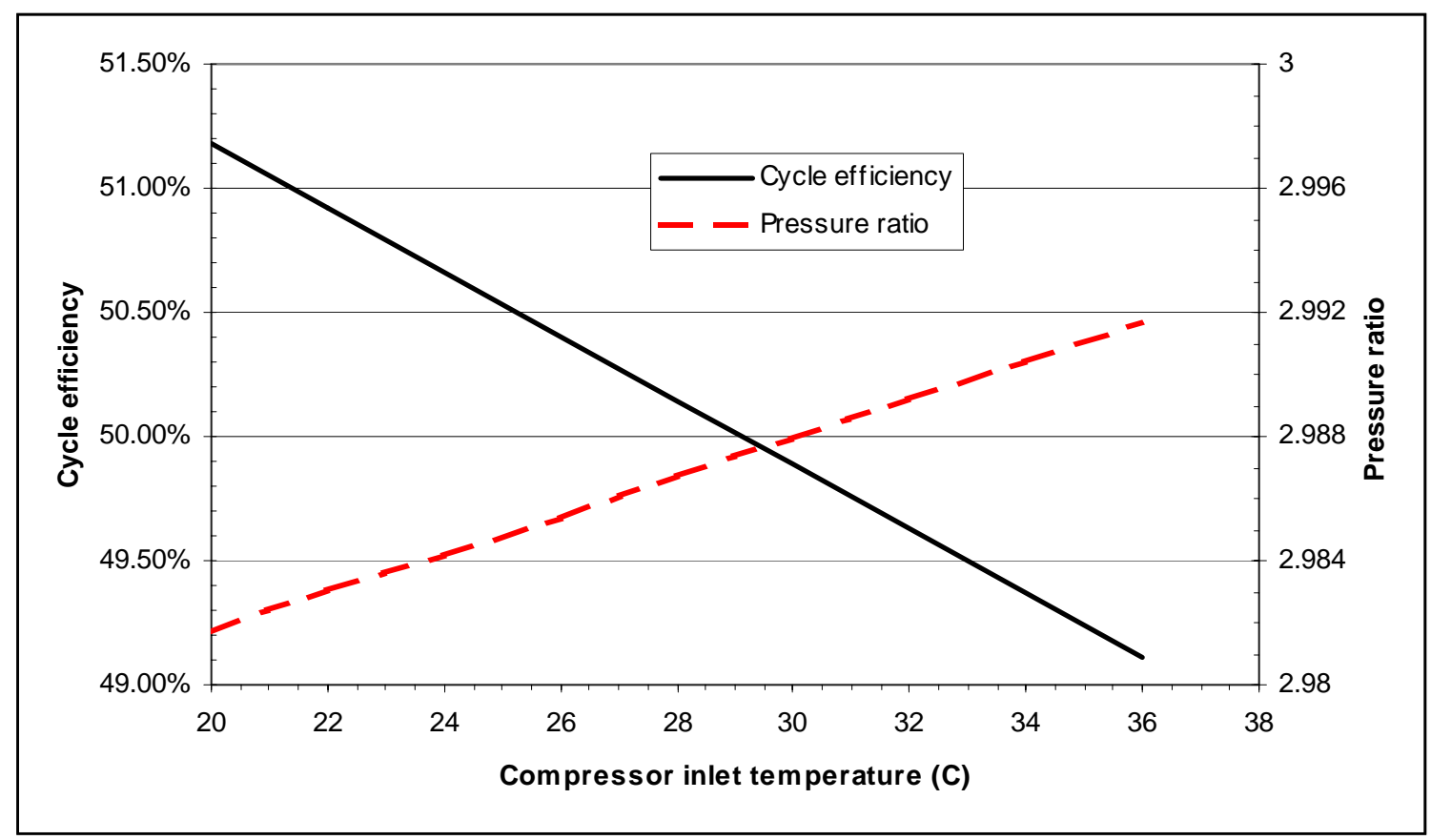

Figure 6.2 The effect of compressor inlet temperature on the cycle performance

\section{- IHX Effectiveness}

As the component directly transferring heat from the primary system to the PCU, the IHX plays an important role on the cycle performance. Figure 6.3 shows the cycle efficiency with respect to the cycle pressure ratio as the IHX effectiveness changes. From the figure, one can be seen that higher IHX effectiveness causes higher cycle efficiency. An increase of the IHX effectiveness from $90 \%$ to $95 \%$ results in a cycle efficiency increase of about 0.8 percentage point when the plant is running at the optimum pressure ratio. However, the IHX effectiveness has a significant impact on its size, thus cost. In Section 3, it was shown that the size of the heat exchanger would be increase by around $75 \%$, the weight by around $100 \%$, thus the cost by around $100 \%$ if the effectiveness increases from $90 \%$ to $95 \%$.

\section{- Recuperator Effectiveness}

The recuperator recovers the energy exhausted from the power turbine, thus increases the cycle efficiency. The cycle efficiency for different recuperator effectiveness is shown in Figure 6.4. From the figure, the cycle efficiency undergoes a considerable increase as the recuperator effectiveness rises in the low cycle pressure ratio range. As the pressure ratio increases, the importance of the recuperator effectiveness decreases. The reason is explained as follows. For a given power turbine inlet temperature, the temperature of turbine exhaust gas is high at low pressure ratios. Then the temperature differential between the turbine exit helium and high-pressure compressor discharge helium is large. Therefore, the gain in recovering the turbine exhaust heat is significant. In contrast, in the high pressure ratio 
range, the temperature differential is small, which results in a cycle efficiency that is insensitive to the recuperator effectiveness.

The recuperator will also be a compact heat exchanger; the relationship of size and effectiveness is the same as for the IHX.

\section{- Turbomachine Efficiency}

As with the power conversion components, the efficiencies of the turbine and compressor also play important roles in cycle performance. Figure 6.5 and Figure 6.6 show the effect of the turbine and compressor efficiencies on the cycle efficiency. Both for turbine and compressor, an efficiency increase of 2 percentage points causes a cycle efficiency increase of approximately 1 percentage point. The maximum efficiency achievable for turbomachines is determined by the manufacturers technology. For the current technology, $90 \%$ polytropic efficiency for the compressor can be achieved while $92 \%$ is possible for the turbine.

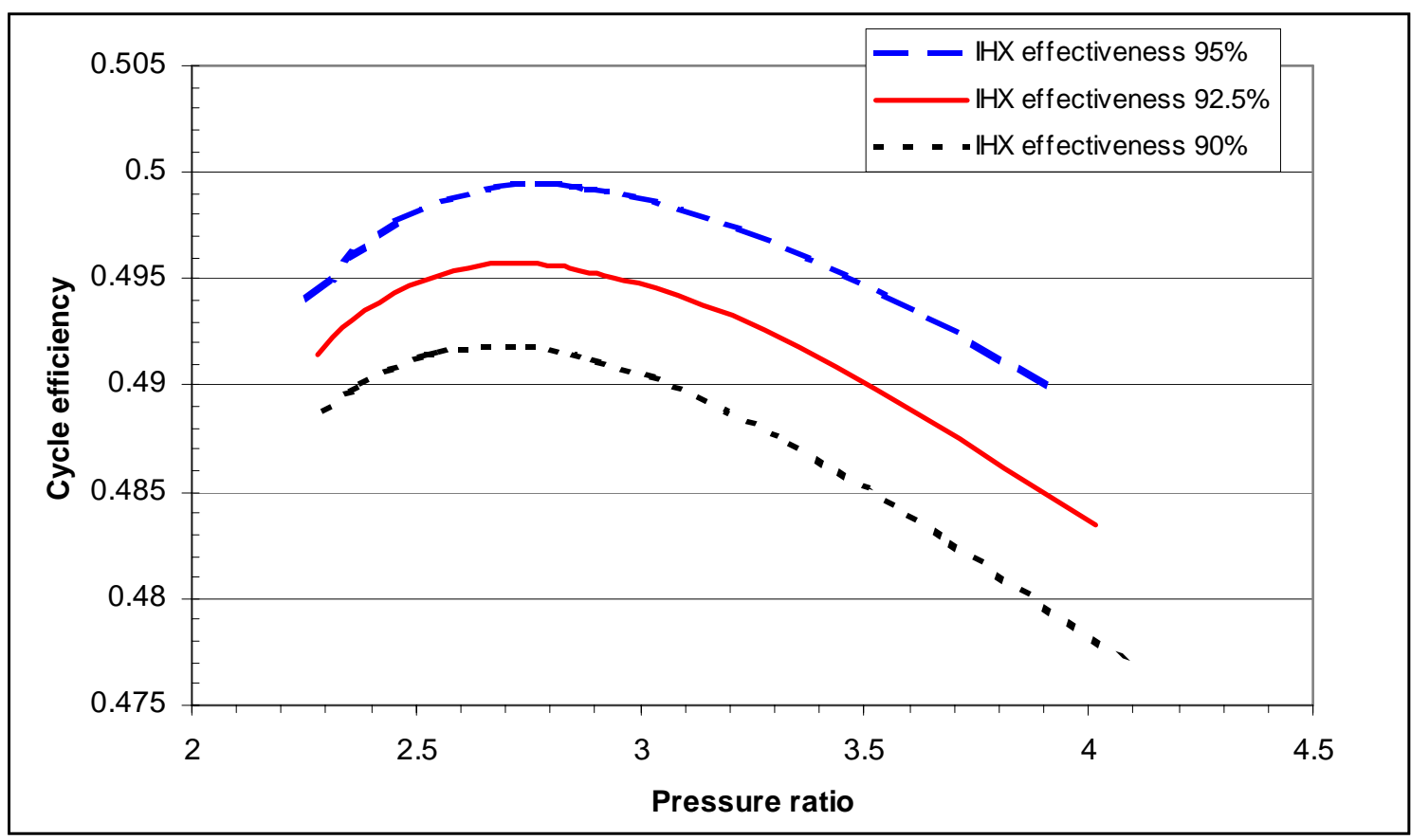

Figure 6.3 Effect of IHX effectiveness on the overall cycle efficiency 


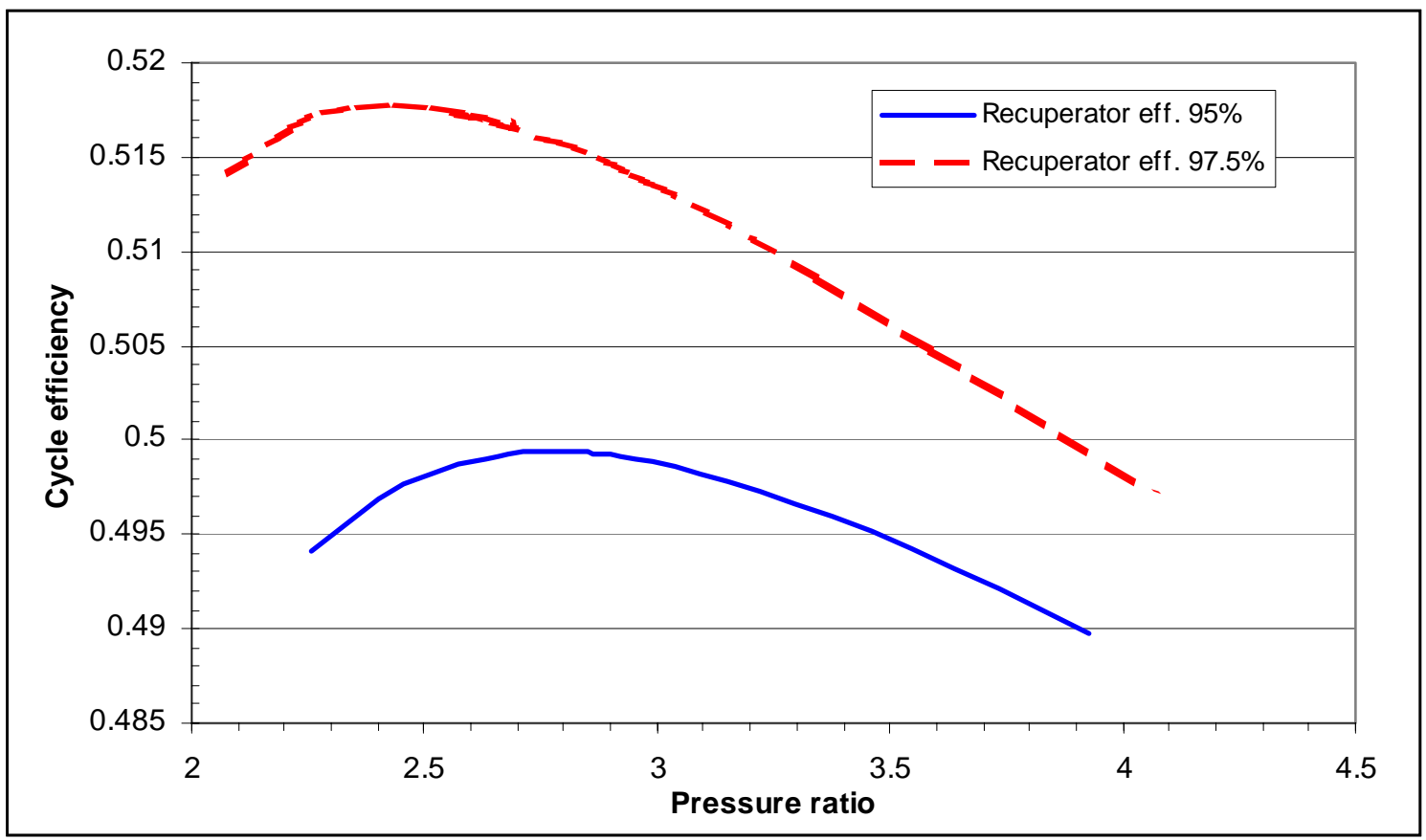

Figure 6.4 Effect of Recuperator effectiveness on the overall cycle efficiency

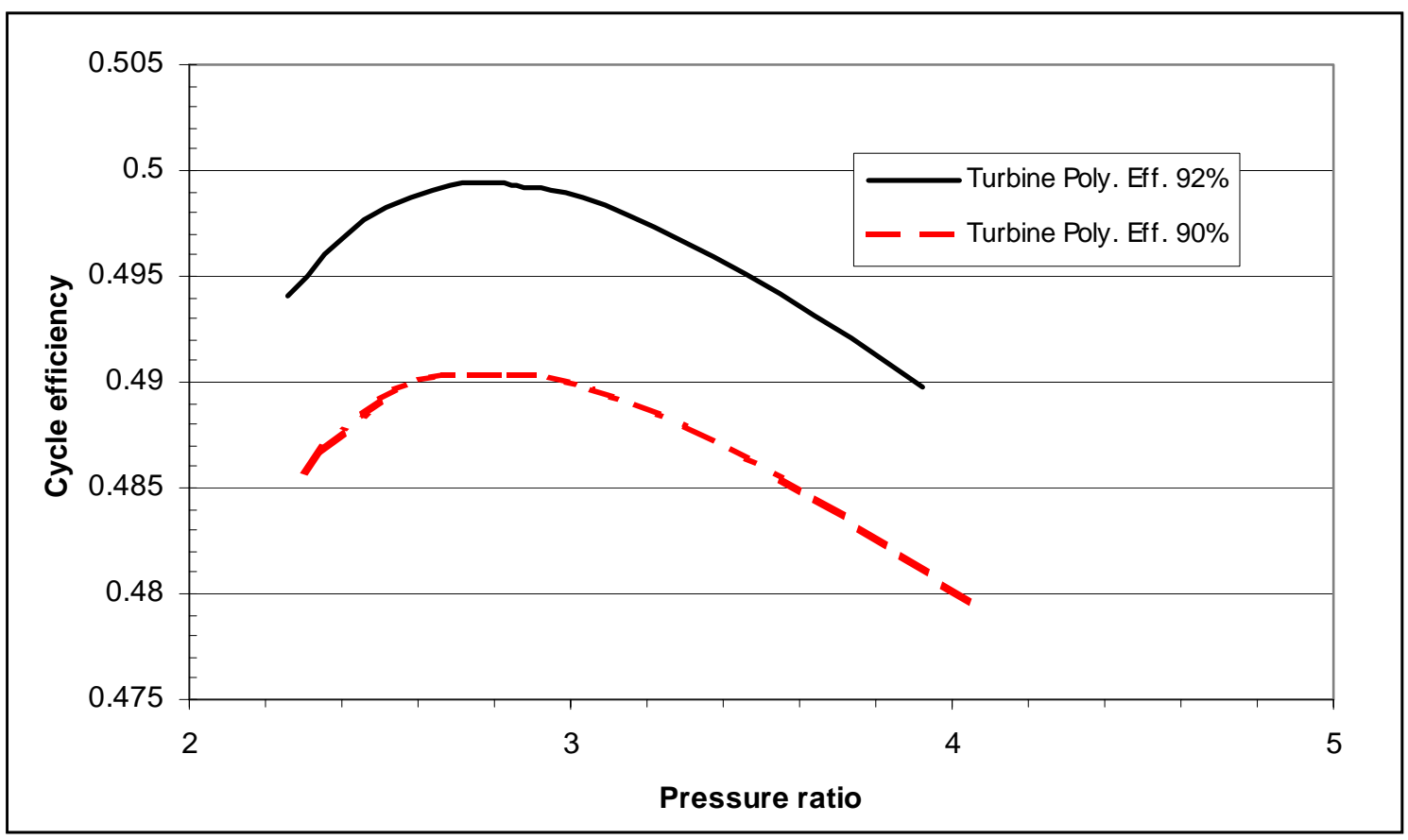

Figure 6.5 Effect of turbine efficiency on the overall cycle efficiency 


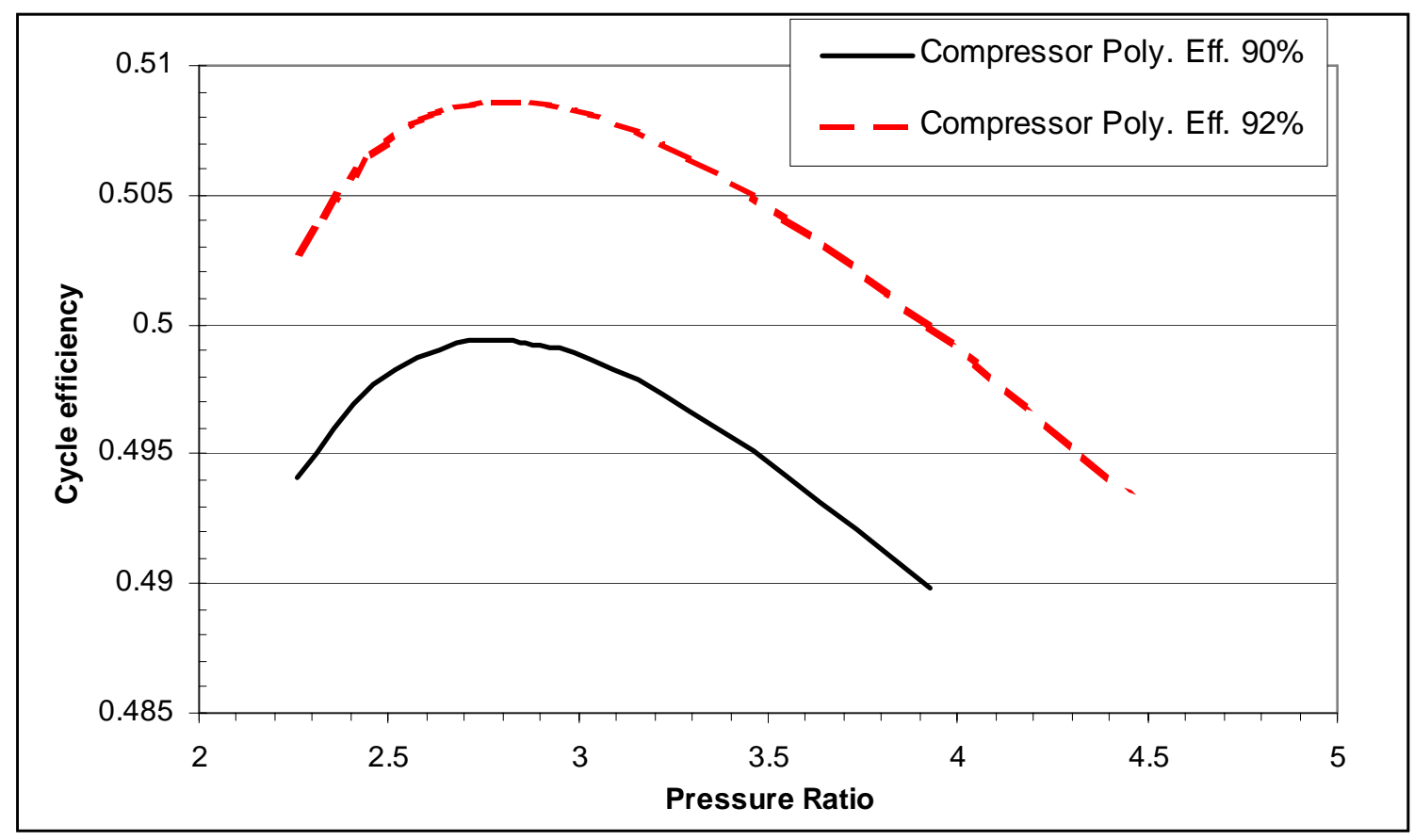

Figure 6.6 Effect of compressor efficiency on the overall cycle efficiency

\section{- Stage Numbers Of Intercooling}

The effect of intercooling stage number is shown in Figure 6.7, which displays the results of non-intercooled, 1 intercooling stage and 3 intercooling stages. A $0.8 \%$ helium side pressure drop for each intercooler is assumed. It can be observed that the benefit of cycle efficiency with more intercooling stages is large in the high pressure ratio range, and the benefit becomes less and less as the pressure ratio decreases. When the pressure ratio decreases below a specific value, the cycle efficiency of the intercooled cycle is even less than that of a non-intercooled one. The reason is that there are pressure losses associated with the intercoolers, and the pressure losses result in a reduction of cycle efficiency. Thus the benefit of cycle efficiency obtained by intercooling is offset by the intercooler pressure losses when the pressure ratio is lower than a specific value. From the figure, it also can be seen that the cycle efficiency increase is remarkable for 1-stage intercooling compared with a non-intercooled cycle, however, the effect is less and less if the intercooling stage number increases. Considering the complexity and cost induced by the intercoolers, the intercooling should not be more than 3 stages.

\section{- Cycle Pressure Losses}

Higher cycle pressure losses, both in the primary cycle or the secondary cycle, consume more compressor or circulator work for providing higher working fluid pressure head, thus leading to lower cycle efficiency. On the contrary, reducing the cycle pressure losses increases the cycle efficiency. As indicated in Section 4, the cycle pressure losses consist of the losses in the components and the losses of the piping connecting them. Table A-1 of Appendix A lists three recuperator design results which possess different pressure losses but 
with the same effectiveness of 95\%. The pressure losses are as follows: $0.8 \%$ (hot side) and $0.13 \%$ (cold side); $1.4 \%$ (hot side) and $0.23 \%$ (cold side); $2 \%$ (hot side) and $0.33 \%$ (cold side). Figure 6.8 shows the cycle maximum efficiencies using three recuperator designs while other cycle parameters remain constant.

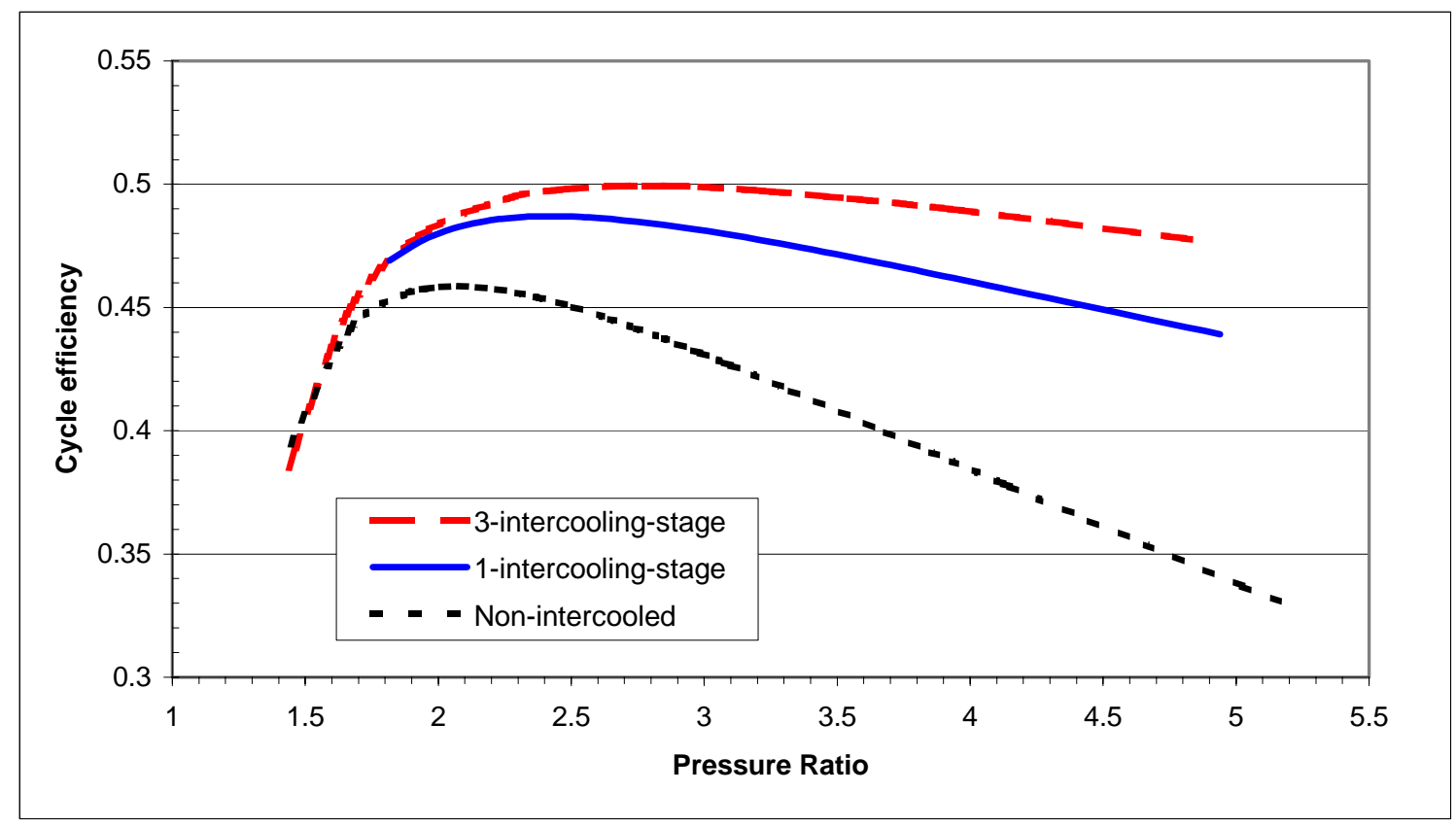

Figure 6.7 Intercooling stage number effect on the cycle efficiency

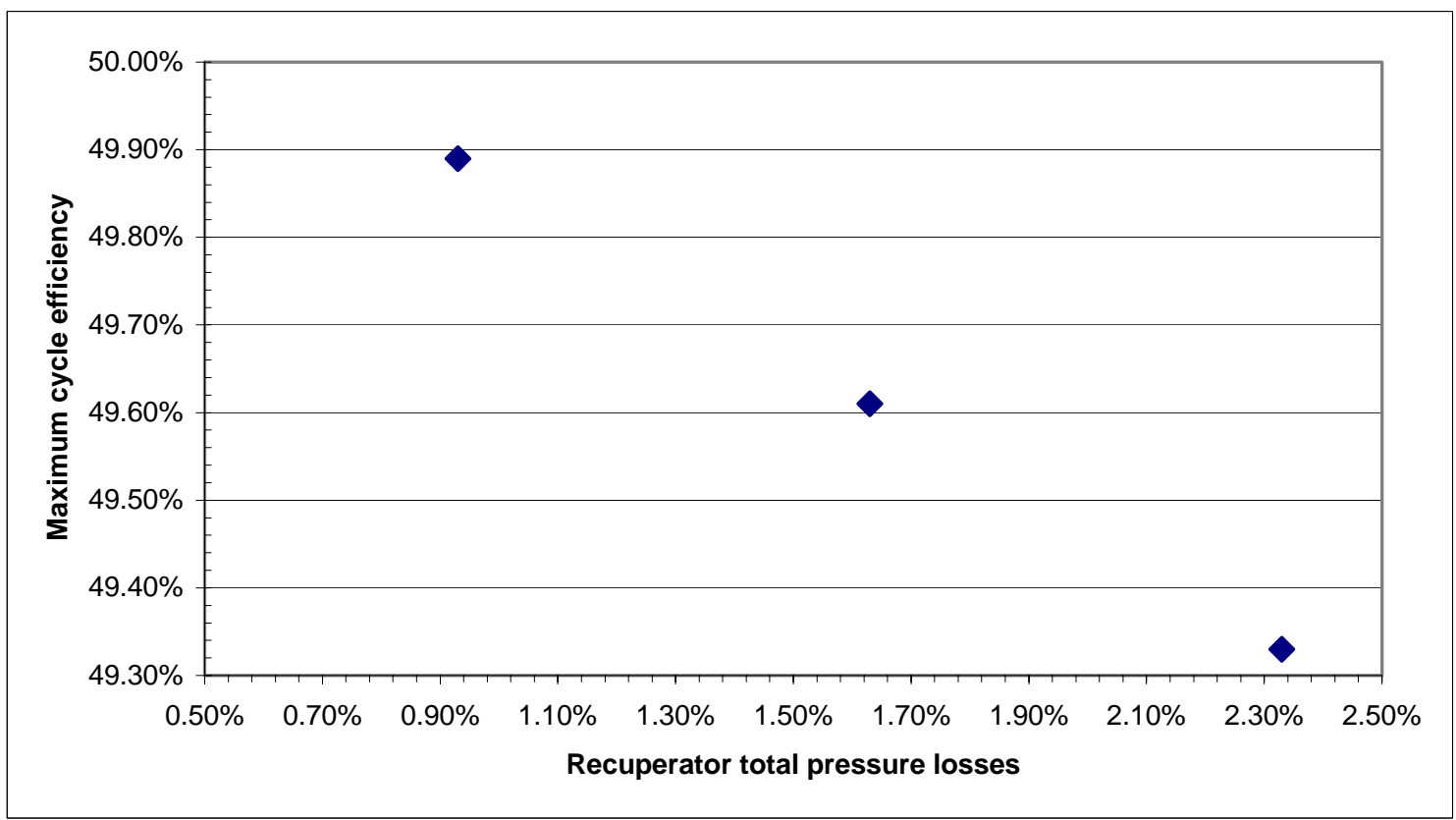

Figure 6.8 Cycle efficiency in three recuperator design conditions 
The pressure losses in a component evolve with its size. Taking the heat exchanger as an example, the one with lower pressure losses possesses a larger size, and thus heavier weight, and higher cost under the condition that the effectiveness remains constant. From Table 3.7 of Section 3, we can see that the design with lower pressure losses possesses larger size and heavier weight while the effectiveness remains constant.

\section{- Bleeding Helium From Compressors}

As described in Section 4.2.1, in practice, 1 2\% mass flowrate of total compressed gas is required to be bled off for cooling the turbine disc and blade roots. In the three-shaft arrangement, we assume bleeding the HP compressor outlet helium to cool the HP turbine discs and blade roots while using MP compressor \#1 outlet helium for the LP turbine and power turbine. The effect of bleed helium amount on the maximum cycle efficiency is shown in Figure 6.9. It can be seen that there is a linear relation between the maximum cycle efficiency and the amount of bleeding helium. Bleeding $1 \%$ of total mass flowrate both from the HP compressor and MP compressor \#1 reduces the maximum cycle efficiency by 0.21 percentage point.

\section{- Sensitivity Analysis Results}

To reveal the relative importance of the cycle parameters around the design point, the results in the previous figures are summarized in Figure 6.10. Figure 6.10 shows the cycle efficiency change resulting from a small percentage change from the design value for a specific parameter, while maintaining other parameters at the design value. The design value for each parameter is given in the parentheses on the line label. From Figure 6.10, the relative degree of sensitivity among the parameters is easily obtained. The order of sensitivity, from the most to the least, is as follows: (1) Recuperator effectiveness, (2) Compressor polytropic efficiency; (3) Turbine polytropic efficiency; (4) IHX effectiveness; (5) Core outlet temperature; (6) Secondary cycle pressure losses; (7) Compressor inlet temperature. It should be pointed out that the cost for improving the component performance by a specific percentage value is different for various components. 


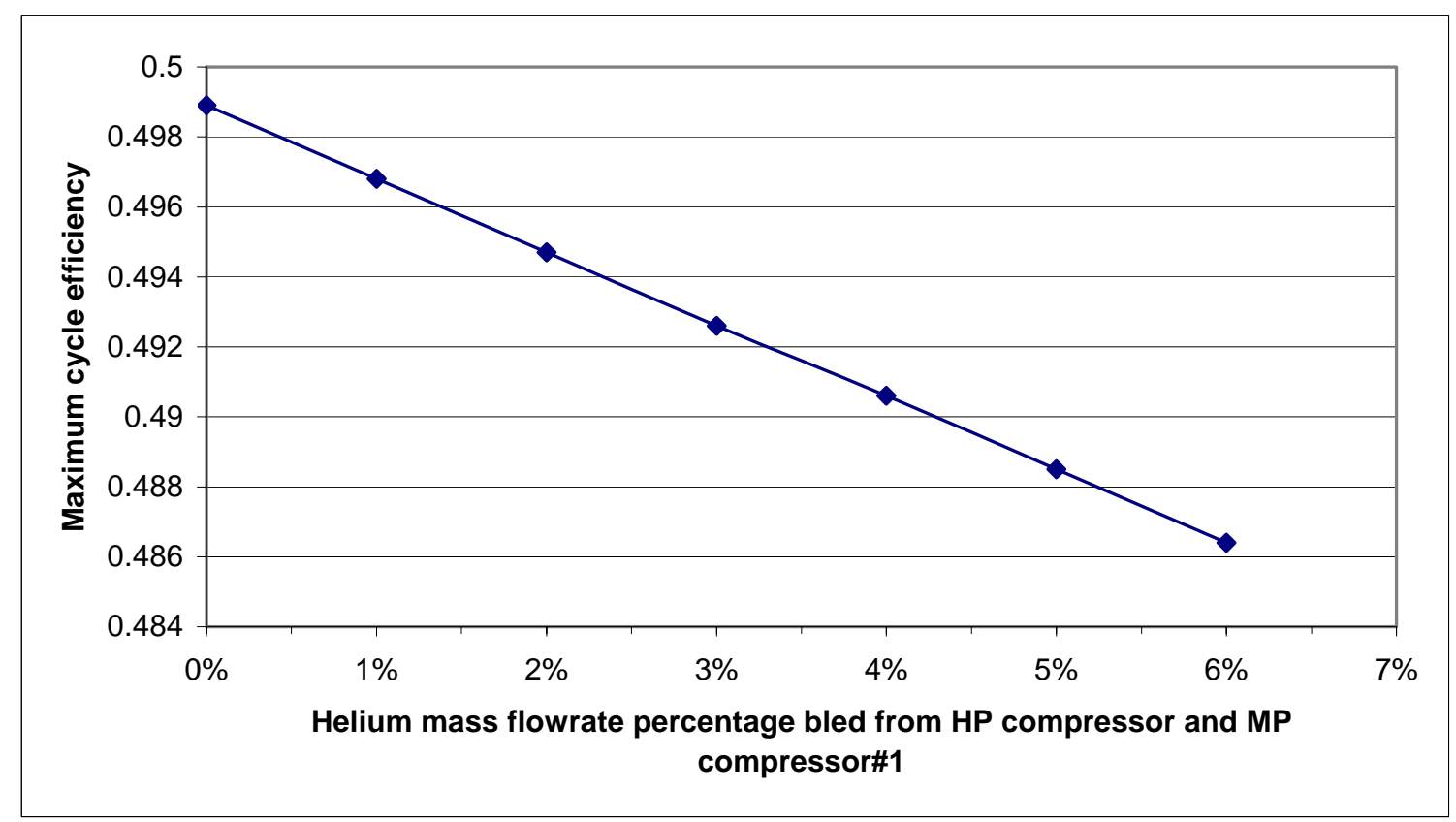

Figure 6.9 The effect on the cycle performance by bleeding helium from the HP compressor and MP compressor \#1 to cool the turbine discs and blade roots

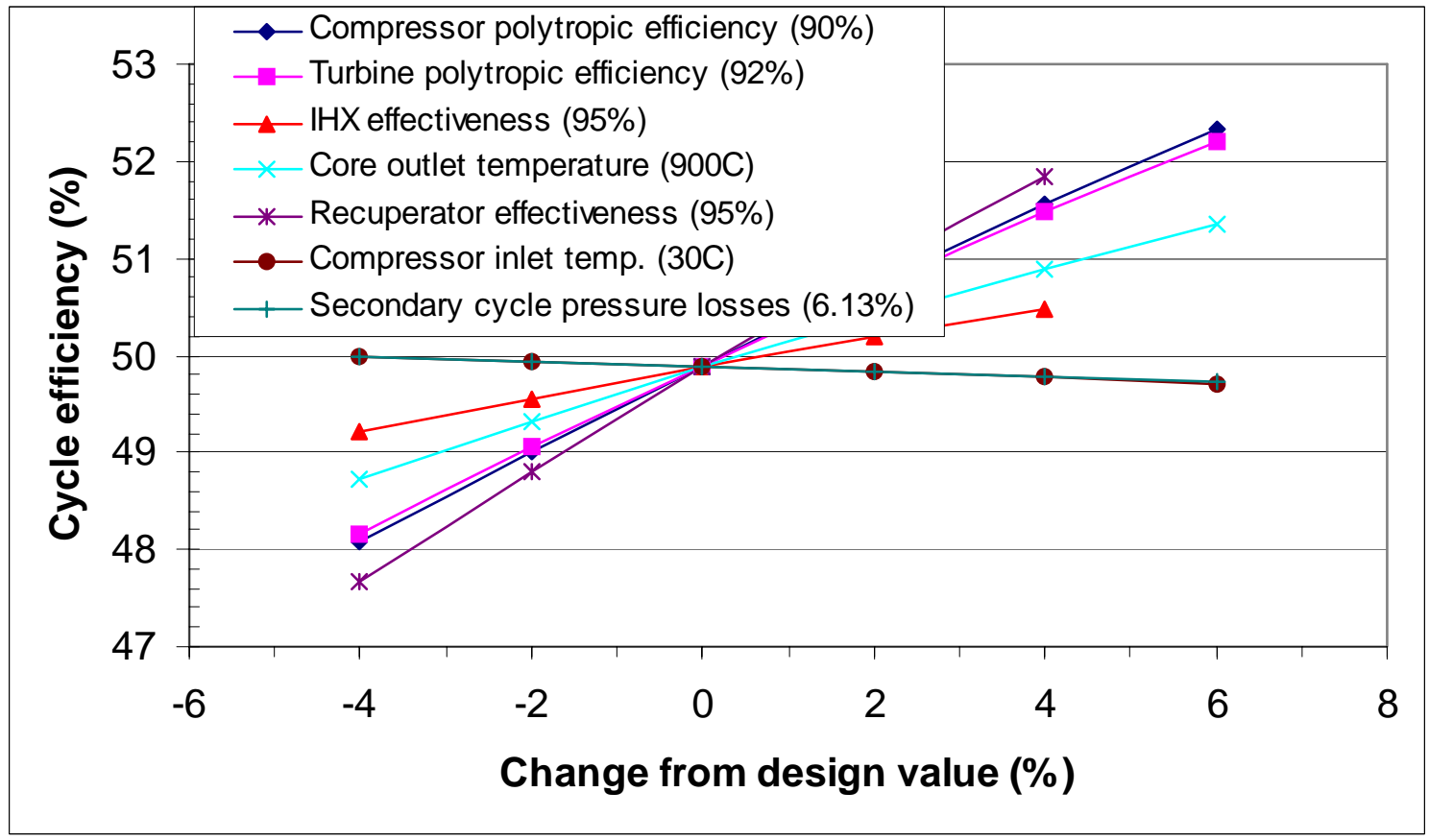

Figure 6.10 Sensitivity of cycle efficiency to component parameters on the cycle

\subsection{Transient analysis}

\subsubsection{Verification With Flownet Model}


In the same way as for the verification of the heat exchanger model, Flownet was also used to verify the PCU part of the dynamic model. Flownet is a general thermal-fluid network analysis code that can model both steady-state and transient flows. It uses nodes and elements to numerically represent the physical components. Its solver, using the implicit pressure correction method, solves for the conservation of mass and energy at all nodes and momentum in all elements. The code can deal with a wide range of network components, for example, turbines, compressors, pipes, valves, heat exchangers. A similar model has been built using Flownet for the MPBR cycle. The schematic of the Flownet model is shown in Figure 6.11. Since the code part for the pebble bed nuclear reactor has not been provided, a pipe (Element 21 in Figure 6.11) is used to represent the reactor core, and its outlet temperature is fixed at $900^{\circ} \mathrm{C}$ at any time. This implies that the core outlet temperature is fixed. Due to the large thermal inertia of the pebble bed reactor core, this assumption is reasonable for a short time period (less than 50 seconds) after transients. The maps of the centrifugal compressors and the maps of the axial turbines are the ones provided by Flownet itself.

For reaching the initial steady state, the inlet pressure of the LP compressor (Node 2) is fixed at $2.71 \mathrm{MPa}$, and the temperatures, pressures and mass flowrates in the water sides of the precooler and intercoolers are all fixed. In the primary side, the pressure of Node 19 is fixed as $8.0 \mathrm{MPa}$. By setting the diameter of the piping in the primary, thus the primary side mass flowrate, the total heat transfer from the primary cycle to the secondary cycle is determined. For a specific total heat, Flownet adjusts the HP turbine shaft speed and LP turbine shaft speed to obtain an equilibrium HP compressor outlet pressure. The initial steady state is balanced with the total heat transfer from the primary cycle to the PCU of 265.7 MW, the PCU maximum pressure of $8.0 \mathrm{MPa}$ and mass flowrate $136.4 \mathrm{~kg} / \mathrm{s}$. The total transferred heat and mass flowrate are slightly higher than the MPBR cycle design values of $257.9 \mathrm{MW}$ and $126.7 \mathrm{~kg} / \mathrm{s}$. However, the small differences of the steady state parameters will not affect the cycle dynamic characteristics. In the Flownet model, a PI controller is used to adjust the bypass valve opening diameter via sensing the power turbine shaft speed. The proportional gain and the integral time are 0.035 and 0.008 , respectively.

In MPBRSim, the inlet temperatures of the compressors are set as constant at $30^{\circ} \mathrm{C}$, which assumes that the cooling capacity of the precooler and intercoolers is very large. A PI controller is also adopted to adjust the bypass valve opening area according to the power turbine shaft speed. The transient response of a control system usually exhibits damped oscillations before reaching a new steady state. These will be demonstrated in the simulation results.

Two models were used to simulate a load transient - electric load $20 \%$ step reduction in 1 second. During plant operation, the generator generates electricity to meet the utility requirement. As the electric load decreases, the excess power produced by the power turbine drives the turbine-generator shaft speed up. After the PI controller senses the power turbine shaft is over the setpoint $3600 \mathrm{rpm}$, the actuator opens the bypass valve to decrease the mass flowrate of all the turbines, and to make the power turbine shaft speed back to the setpoint. The calculated cycle responses of the two models are shown in the figures from 6.12 to 6.18 . 
From the figures, it can be seen that both models demonstrate the same trends of the dynamic characteristics during load transient. After electric load reduction, the speed of the power turbine shaft increases, resulting in a bypass valve mass flowrate rise. The speeds of the HP turbine shaft and the LP turbine shaft both decrease; The pressure of the low pressure zone (LP compressor inlet) increases and the pressure of the high pressure zone (HP compressor outlet) decreases, which means the pressure ratio of the cycle decreases. After reaching a new steady state, the speeds of the HP turbine shaft and LP turbine shaft are both lower than their initial values. The inlet temperature of the IHX cold side is higher than its initial value. At the new steady state, the speeds of the HP shaft and LP shaft in the Flownet model are about 200rpm higher than that of MPBRSim. The differences of the speeds of the turbine shafts might be caused by using different compressor maps, though they are all centrifugal maps. Generally, the response of MPBRSim is slightly faster than those of the Flownet model. This may be attributed to the ignoring the gas compressibility in the gas volume of the heat exchangers in the MPBRSim.

Based on these results it is appropriate to now extend the model to include the reactor model.

\subsubsection{0\% Load Step Change - Bypass Valve Control Used}

This transient simulates a $10 \%$ load rejection from $100 \%$ with the power conversion system in which the type of compressor is axial. Bypass valve control is used to maintain the speed of the power turbine shaft at $3600 \mathrm{rpm}$. In this simulation, the core fission power is adjusted through the reactivity caused by the fuel temperature change while the control rods have not been used for maintaining the core outlet temperature. Thus, the reactor core outlet temperature varies. For simplicity, the inlet temperatures of the compressors have been kept constant at $30^{\circ} \mathrm{C}$. Figures 6.19 to 6.32 show the response of the plant.

Due to the load reduction, a positive net torque is present on the power turbine shaft, resulting in an increase of the shaft speed. The PI controller sends a signal to the bypass valve actuator to open the bypass valve. As the bypass valve opens, a stream of working fluid bypasses all the turbines and the mass flowrate of the compressor increases. Since less mass flowrate passes through the turbines, the turbine operation points shift from their design points. The shifting of the turbine operation point results in an increase of turbine outlet temperature. From Figure 6.29 we can see that the outlet temperature of the power turbine increases about $23^{\circ} \mathrm{C}$ just after the transient and the increase becomes $17^{\circ} \mathrm{C}$ after reaching new steady state. The combination of the reduced mass flowrate and increase inlet temperature of the secondary side of the IHX makes the heat removed from the primary cycle decrease, as shown in Figure 6.19. As a result, the core inlet temperature increases and the fuel average temperature rises. Because of the temperature coefficient of the reactivity, negative reactivity is induced in the reactor core, which reduces the fission power. The fission power reduction results in a core outlet temperature decreases and the fuel average temperature decreases and approaches the initial value. 


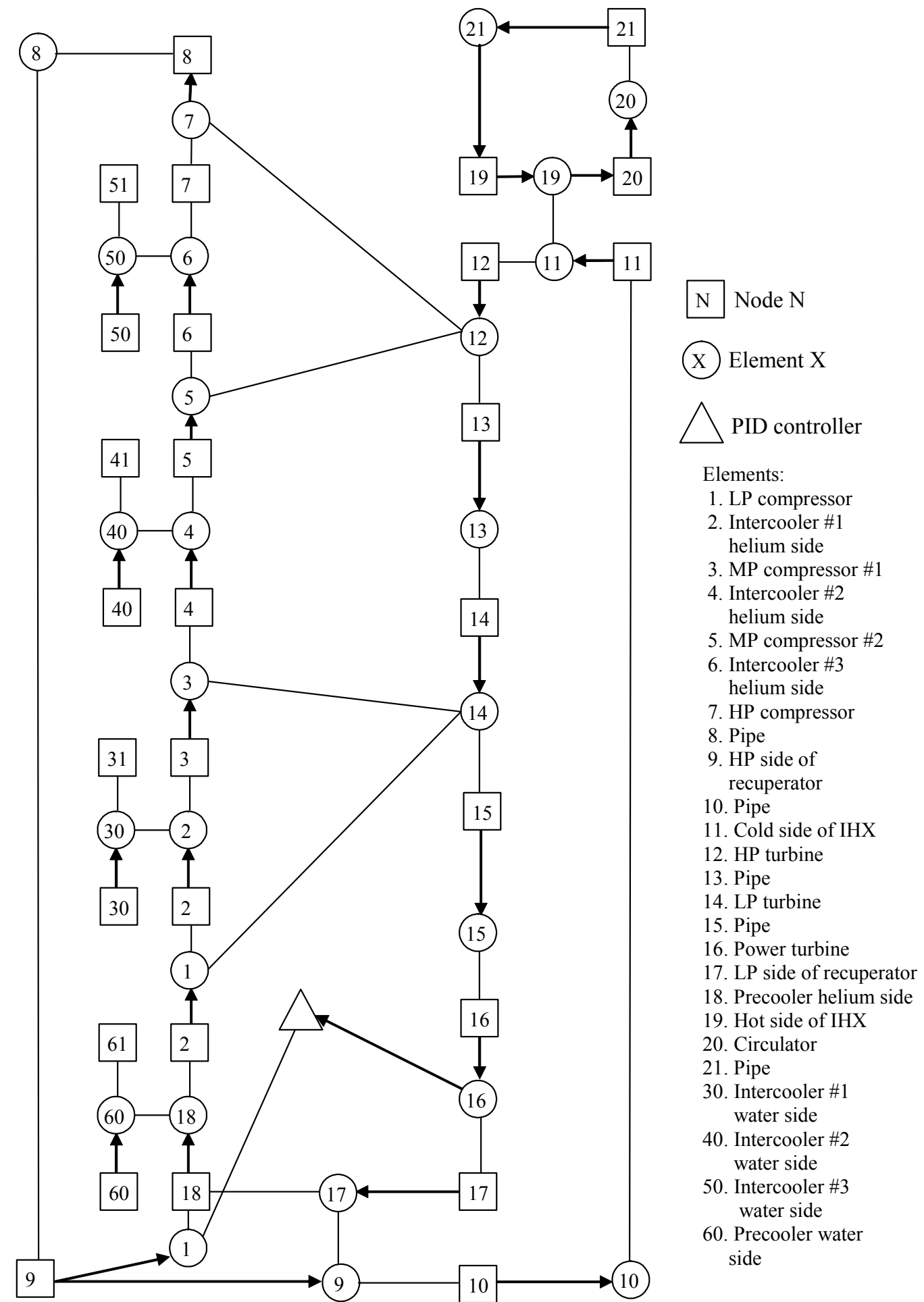

Figure 6. 11 Schematic of the Flownet model 


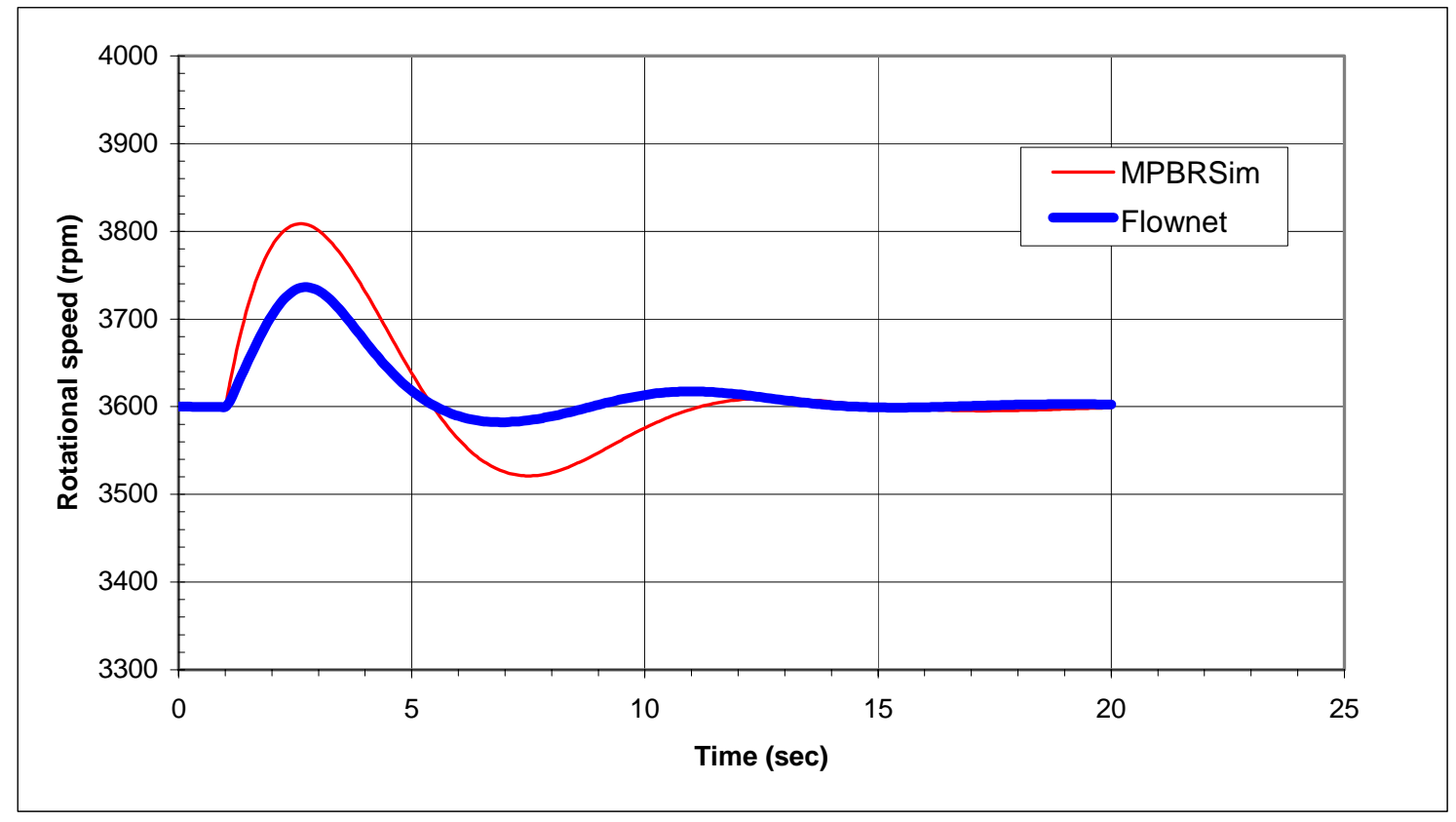

Figure 6.12 Power turbine shaft speed response to $20 \%$ load step reduction, compared to the Flownet model

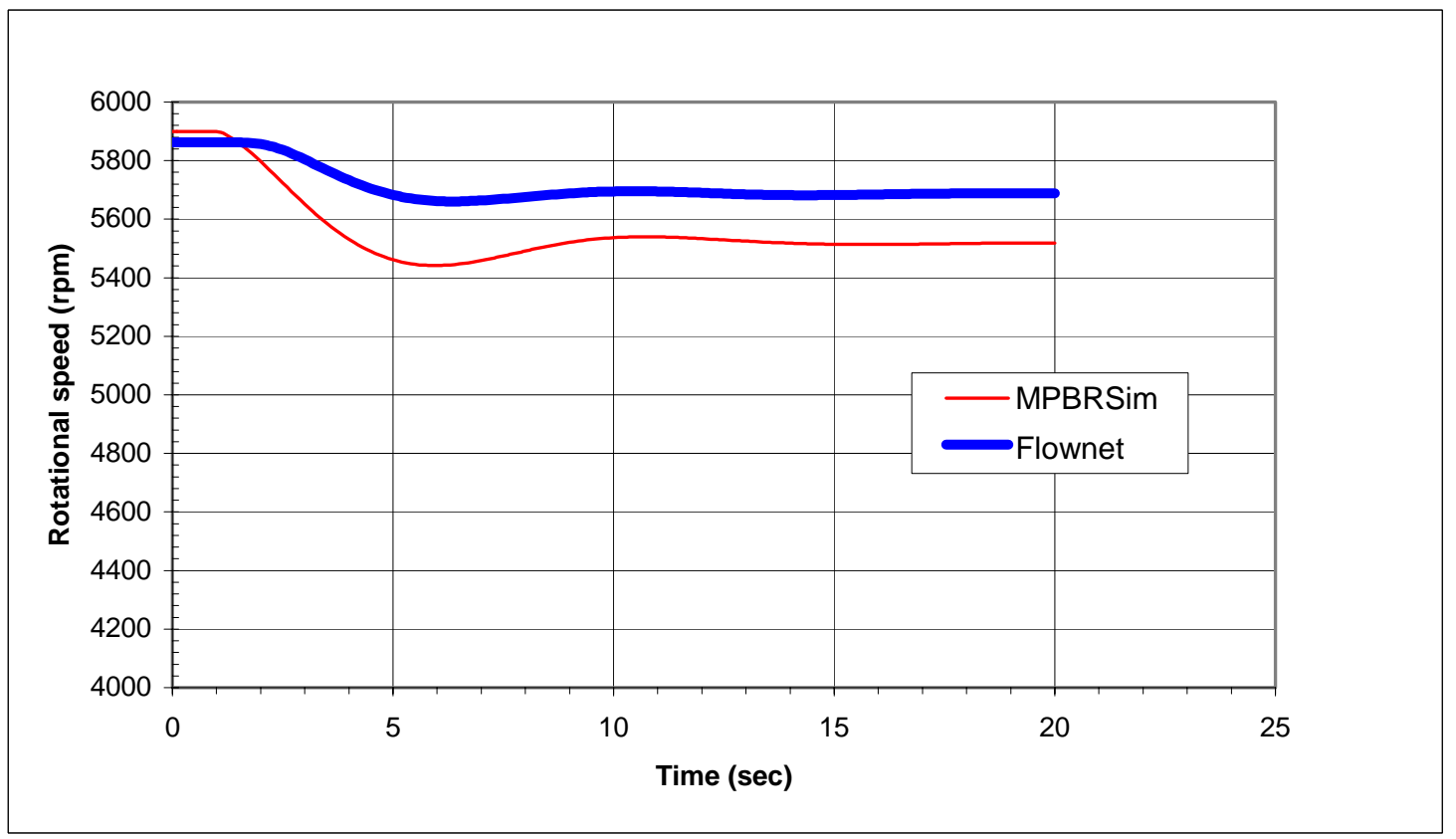

Figure 6.13 HP turbine shaft speed response to $20 \%$ load step reduction, compared to the Flownet model 


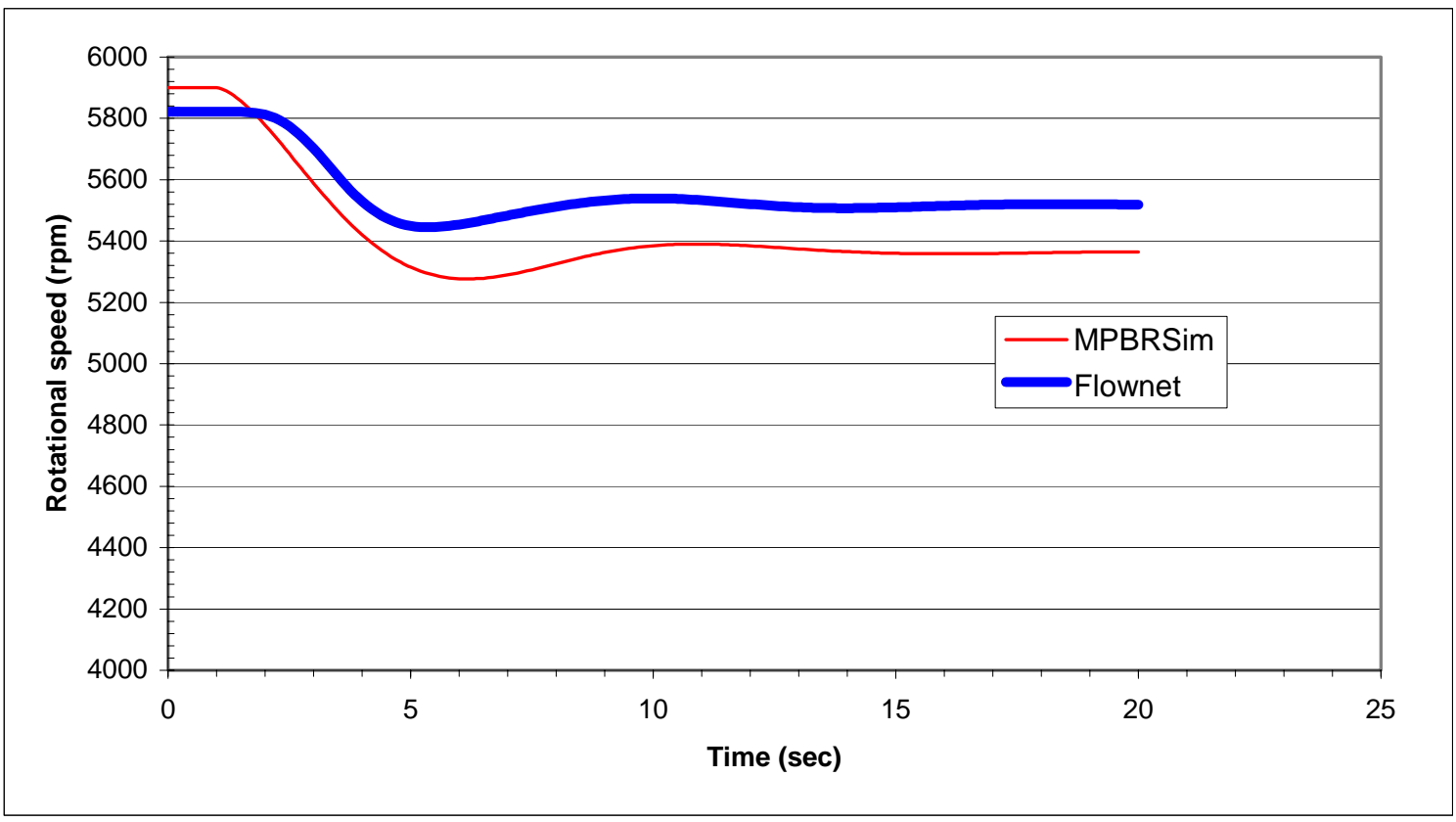

Figure 6.14 LP turbine shaft speed response to $20 \%$ load step reduction, compared to the Flownet model

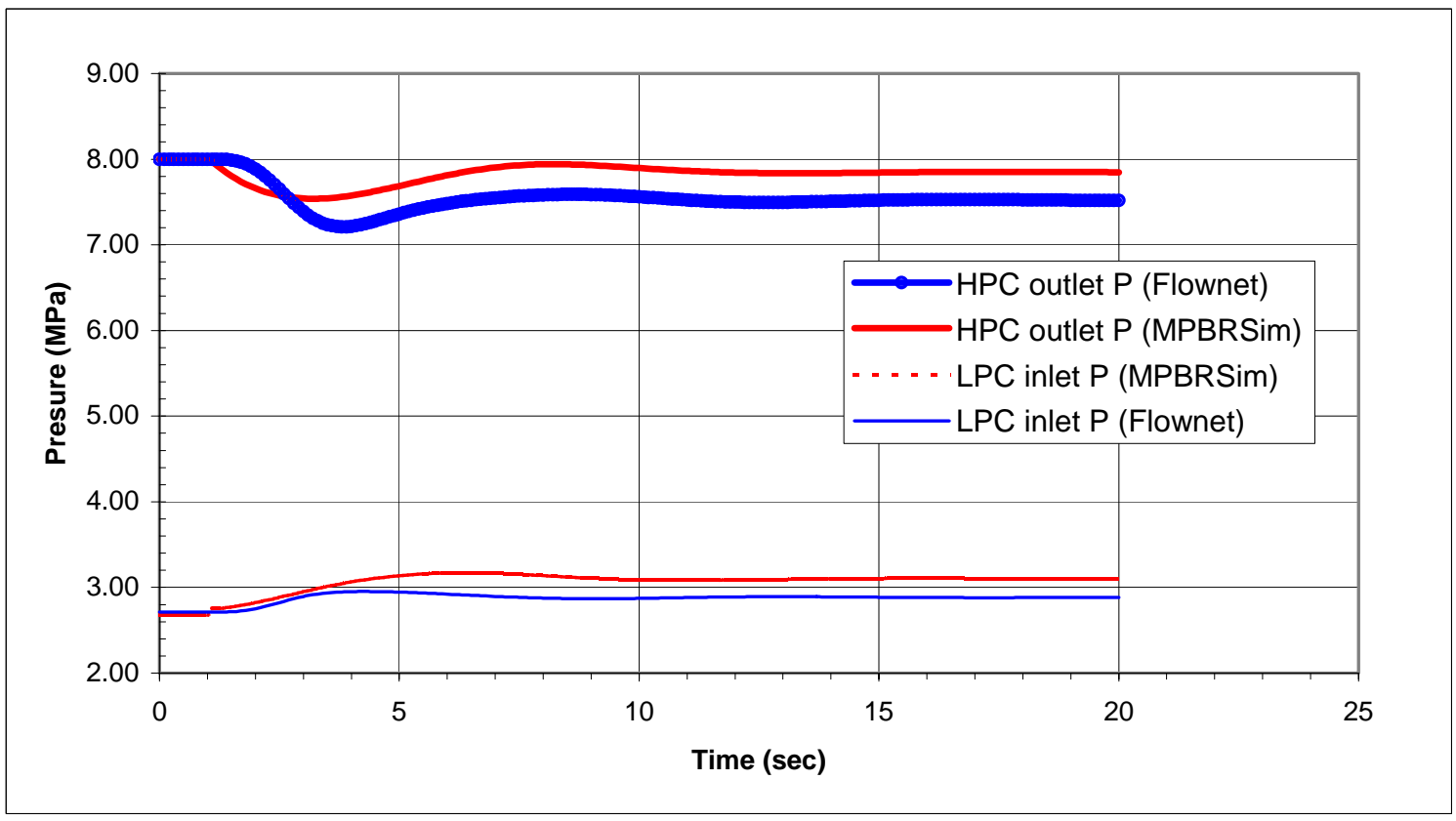

Figure 6.15 Pressures response to $20 \%$ load step reduction, compared to the Flownet model 


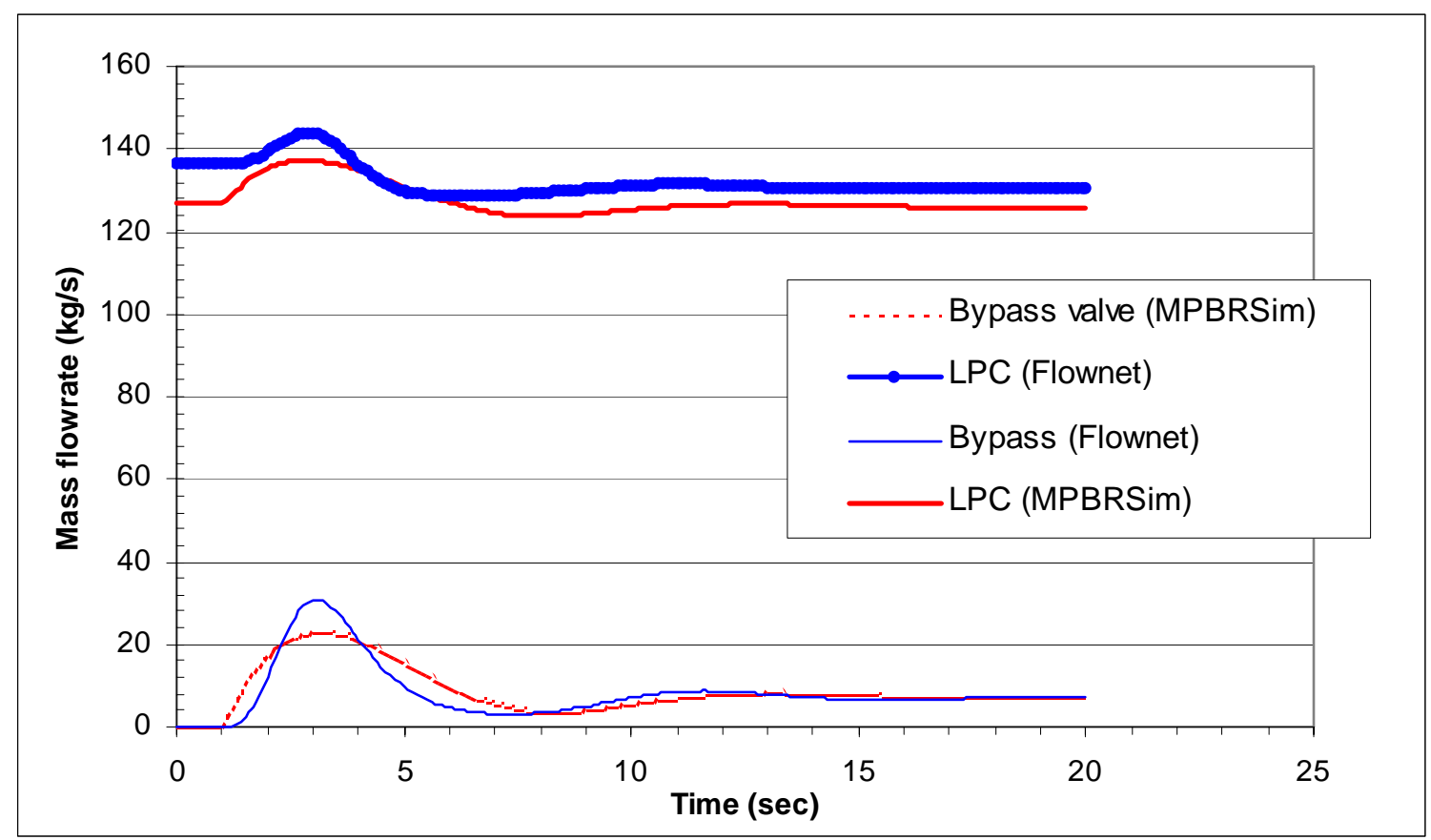

Figure 6.16 Mass flowrate response to $20 \%$ load step reduction, compared to the Flownet model

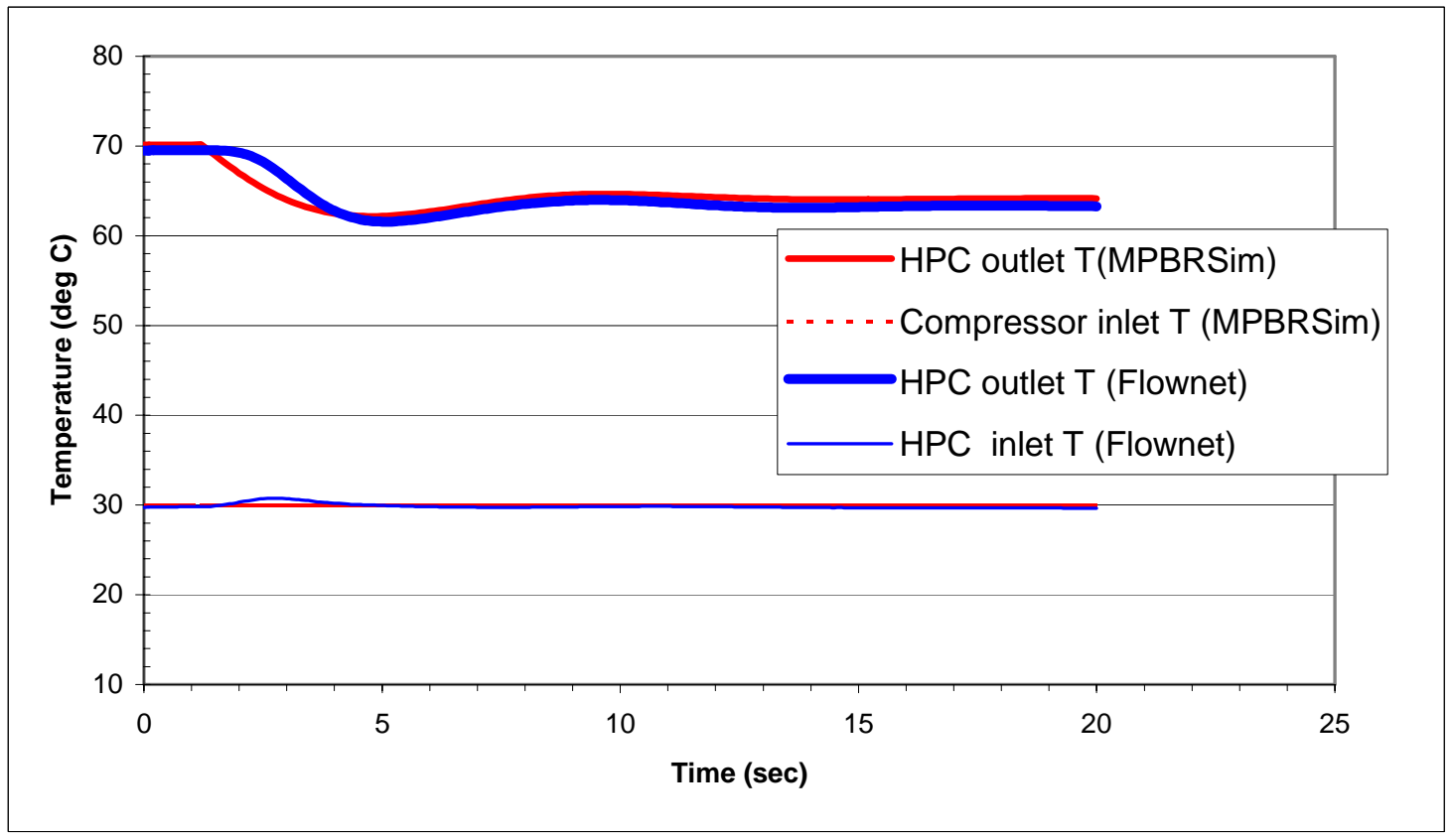

Figure 6.17 Compressors temperatures response to $20 \%$ load step reduction, compared to the Flownet model 


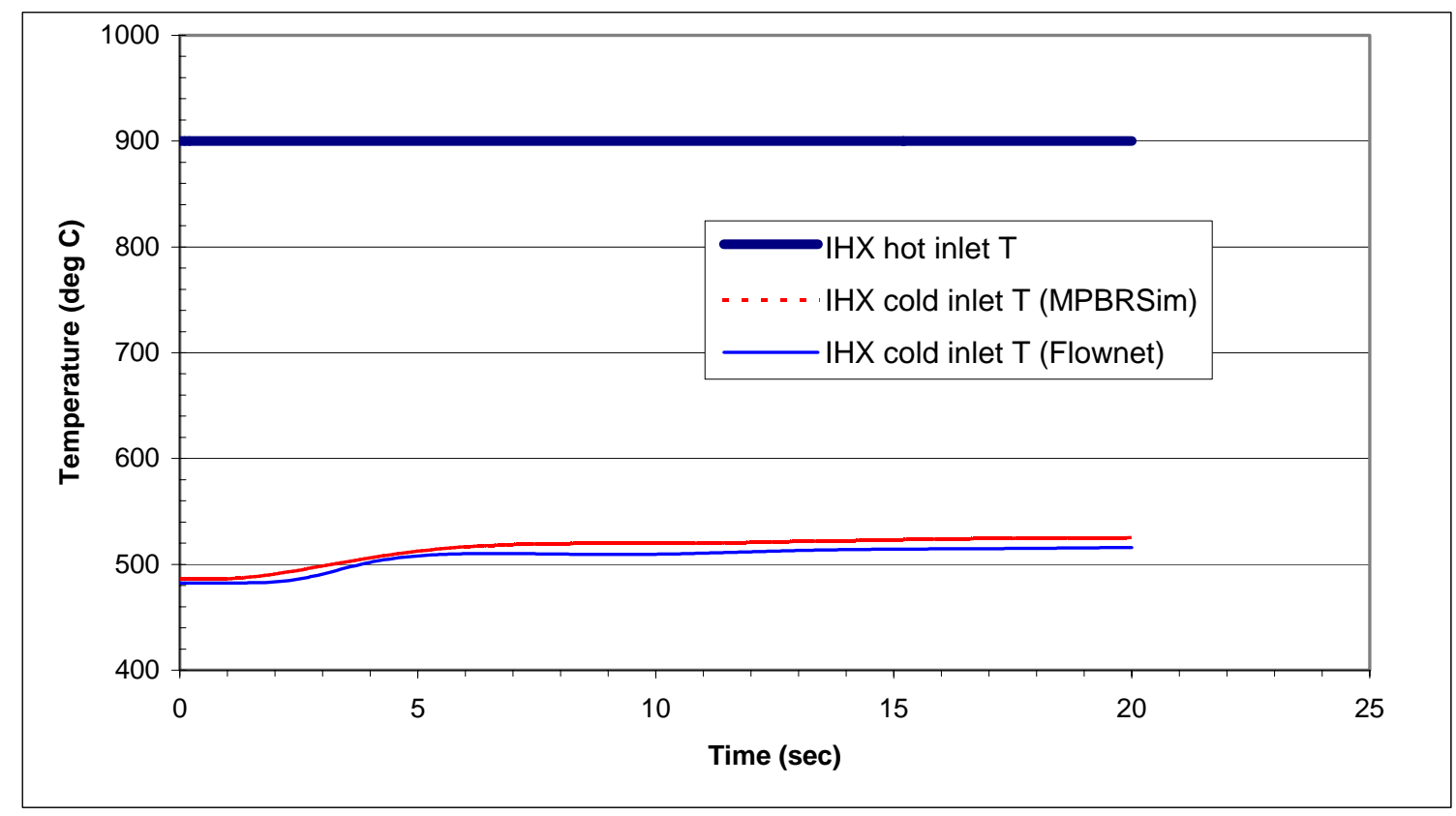

Figure 6.18 IHX temperatures response to $20 \%$ load step reduction, compared to the Flownet model

When the bypass valve opens, the outlet pressure of the power turbine increases; and thus the pressure ratios of turbines decrease. The lower pressure ratio and the reduced mass flowrate make the power output of turbines decrease. For compressors, their mass flowrates increase as the bypass valve opens. As a result, on the HP turbine shaft or LP turbine shaft, the total power of compressors is higher than their corresponding turbine power, a negative net torques is imposed on the shaft, causing its speed decrease as shown in Figure 6.24. After that, due to the slower rotational speed, the compressors consume less power and their outlet temperatures decrease. Figure 6.30 shows the temperatures of the compressors. At the new steady state, since the turbines are not working at their design points, their pressure ratios are lower than the nominal values. The cycle pressure ratio is lower than the nominal one, thus the pressure in the low-pressure zone is higher than its initial value while the pressure in the high-pressure zone is lower than its initial value.

From the figures, we can see that the response of the PCU is much faster than that of the reactor. For example, the speeds of the HP turbine shaft and the LP turbine shaft decrease swiftly to their new steady-state values during the first 20 seconds after the transient while the core outlet temperature remaines around $900^{\circ} \mathrm{C}$ for as long as 40 seconds, shown in Figures 6.24 and 6.27 .

After reaching a new steady state, the bypass valve mass flowrate is about $2.8 \mathrm{~kg} / \mathrm{s}, 2.2 \%$ of the total mass flowrate. The fission power is $91.2 \%$ of the original power level, which means using bypass valve control does not dramatically degrade the cycle efficiency. However, opening the bypass valve results in that the inlet temperature of the low-pressure side of the recuperator increases about $17^{\circ} \mathrm{C}$, and the core inlet temperature increases about $19^{\circ} \mathrm{C}$, causing thermal stress on the recuperator and reactor structures. 


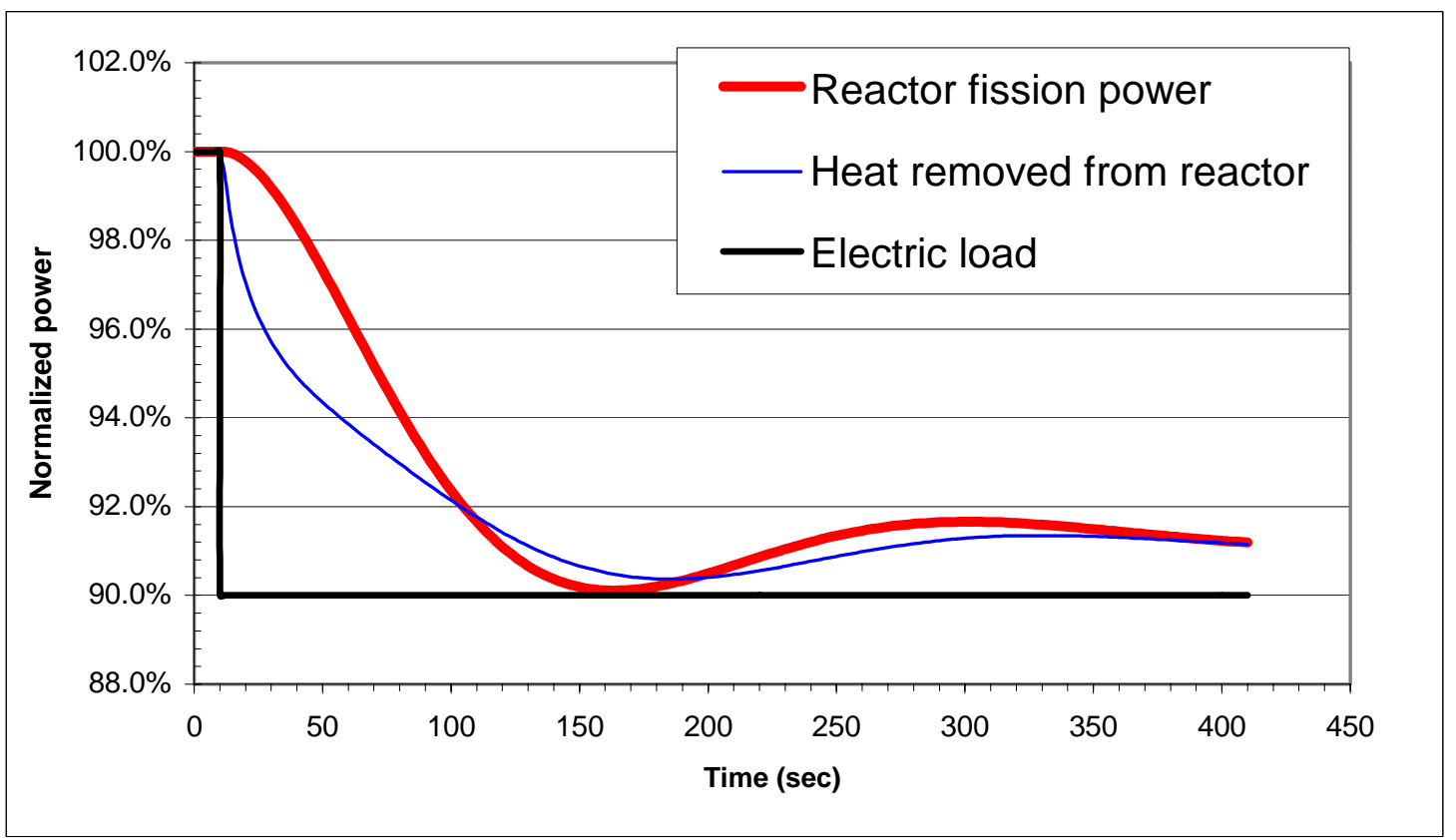

Figure 6.19 Electric load and reactor fission power in 10\% load step reduction with bypass valve control used.

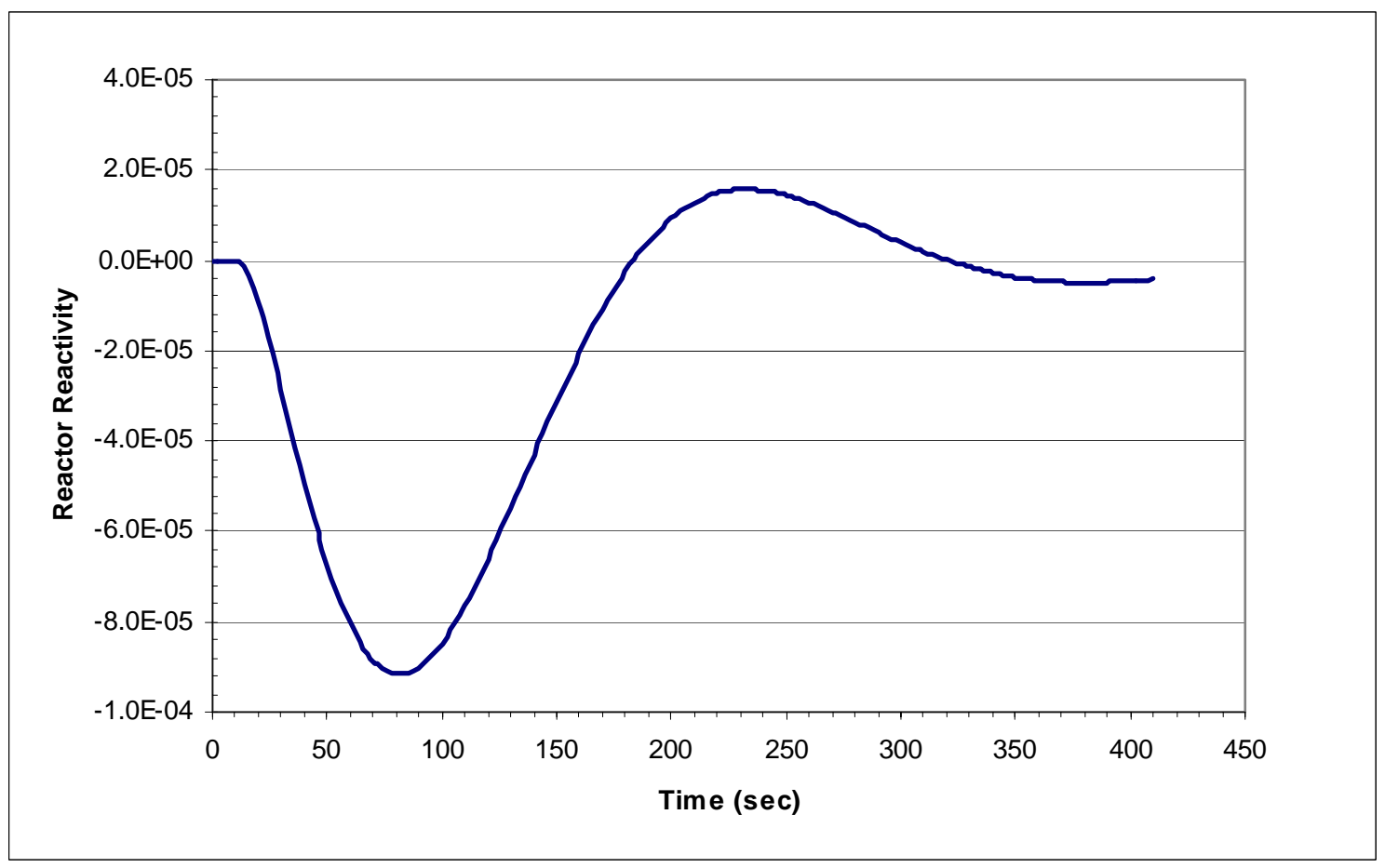

Figure 6.20 Reactor reactivity in $10 \%$ load step reduction with bypass valve control used 


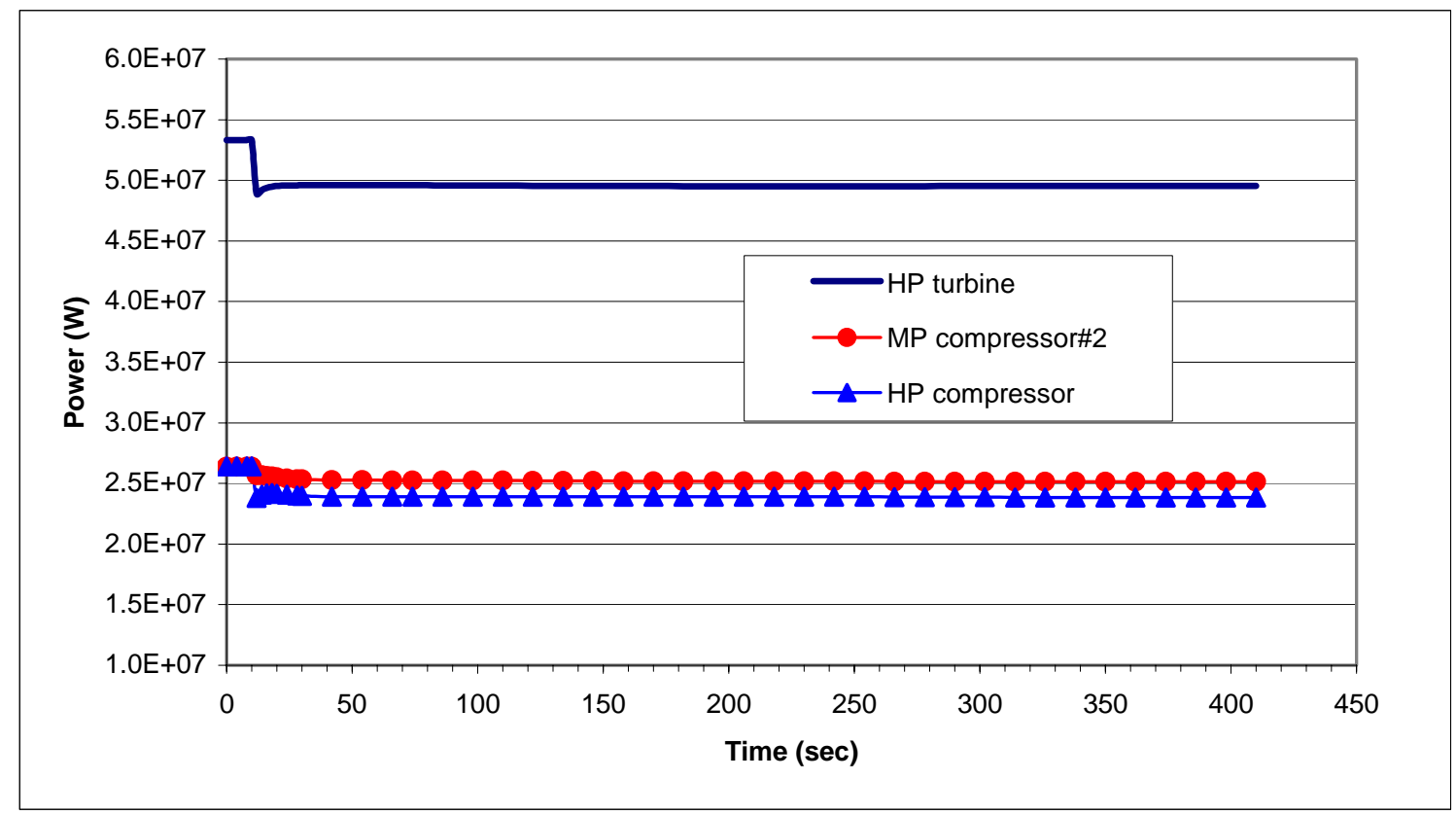

Figure 6.21 Power of components on the HP turbine shaft in 10\% load step reduction with bypass valve used

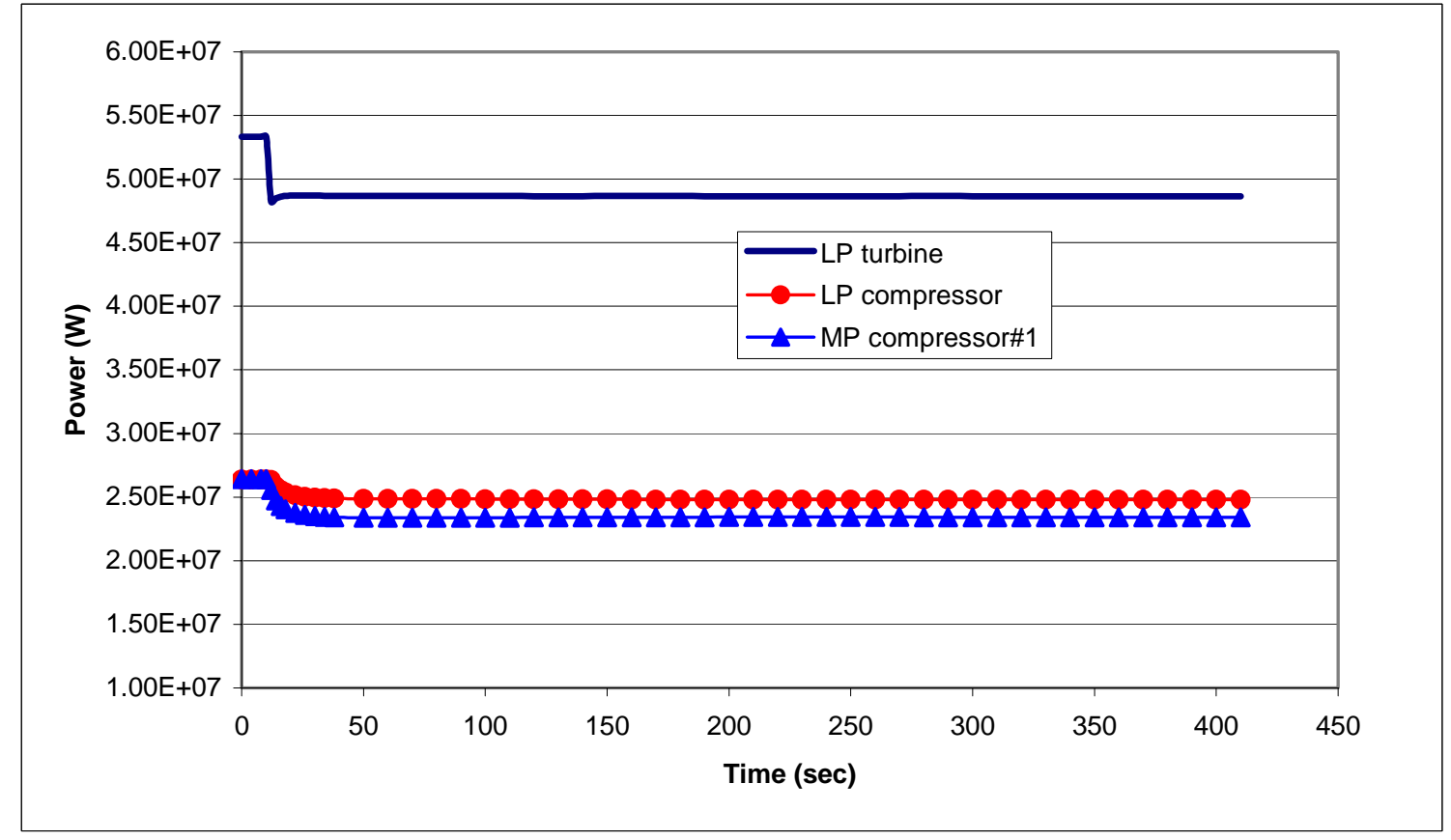

Figure 6.22 Power of components in the LP turbine shaft in 10\% load step reduction with bypass valve control used 


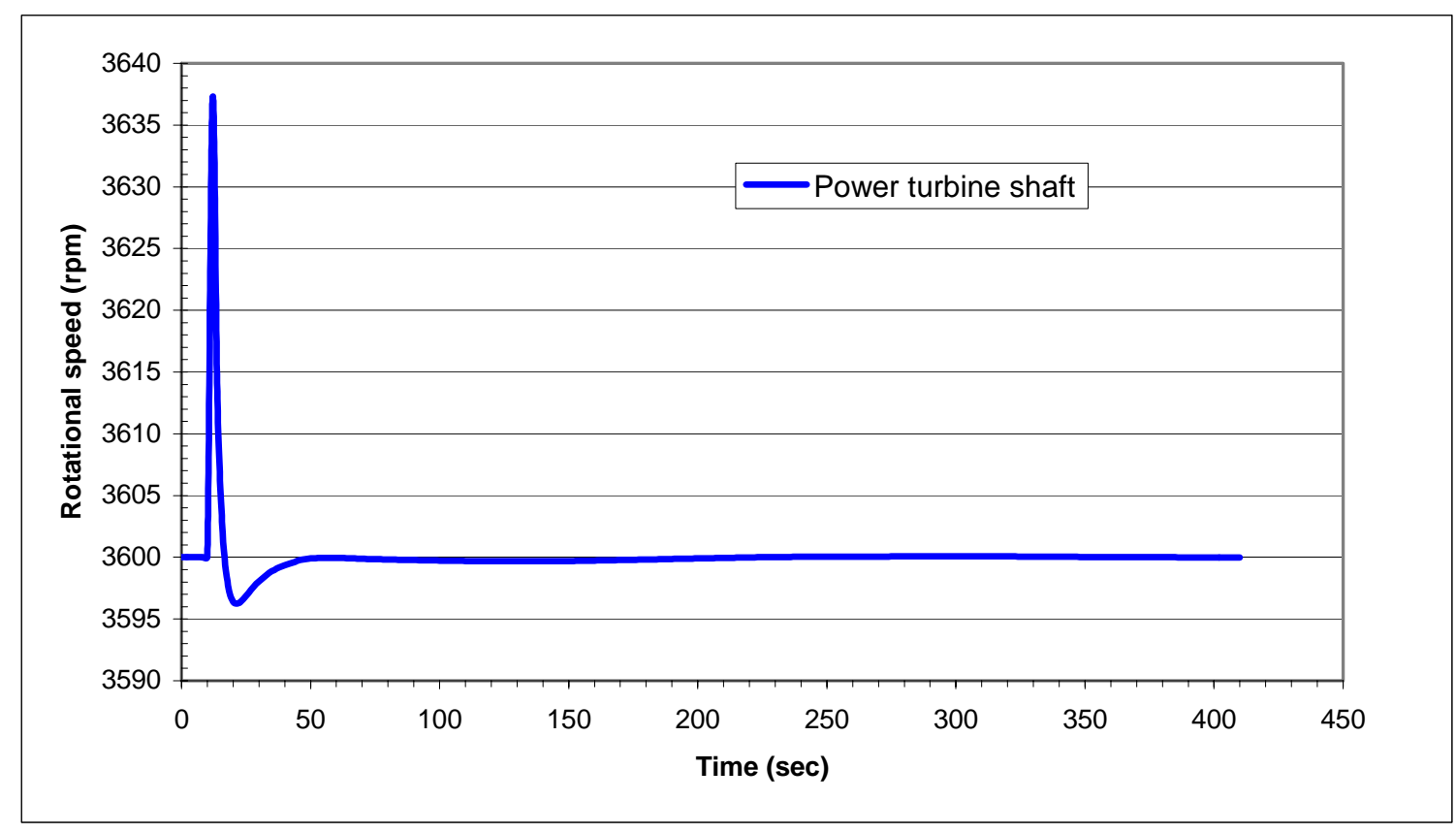

Figure 6.23 Power turbine shaft speed in $10 \%$ load step reduction with bypass valve control used

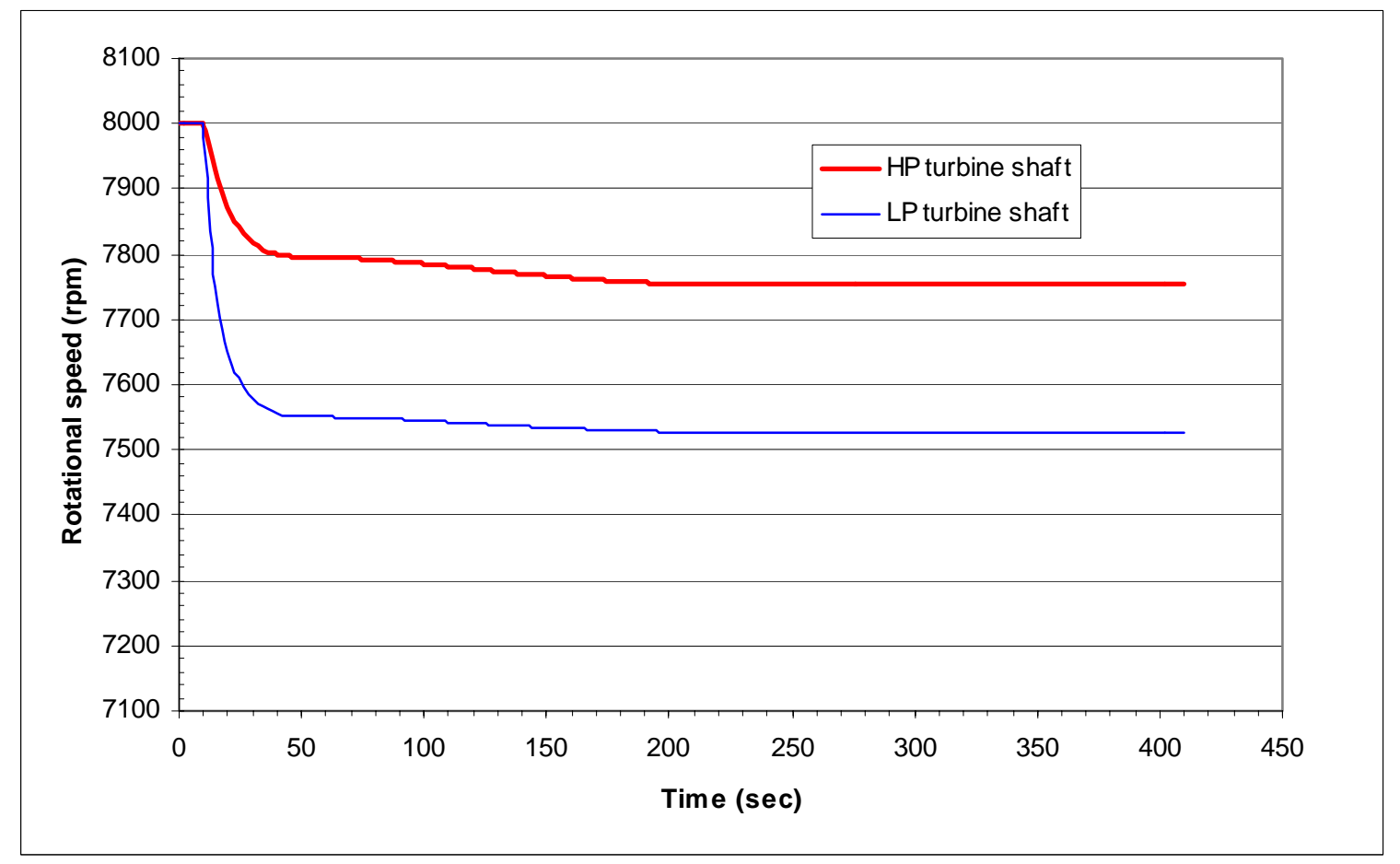

Figure 6.24 Speeds of the HP turbine shaft and the LP turbine shaft in 10\% load step reduction with bypass valve control 


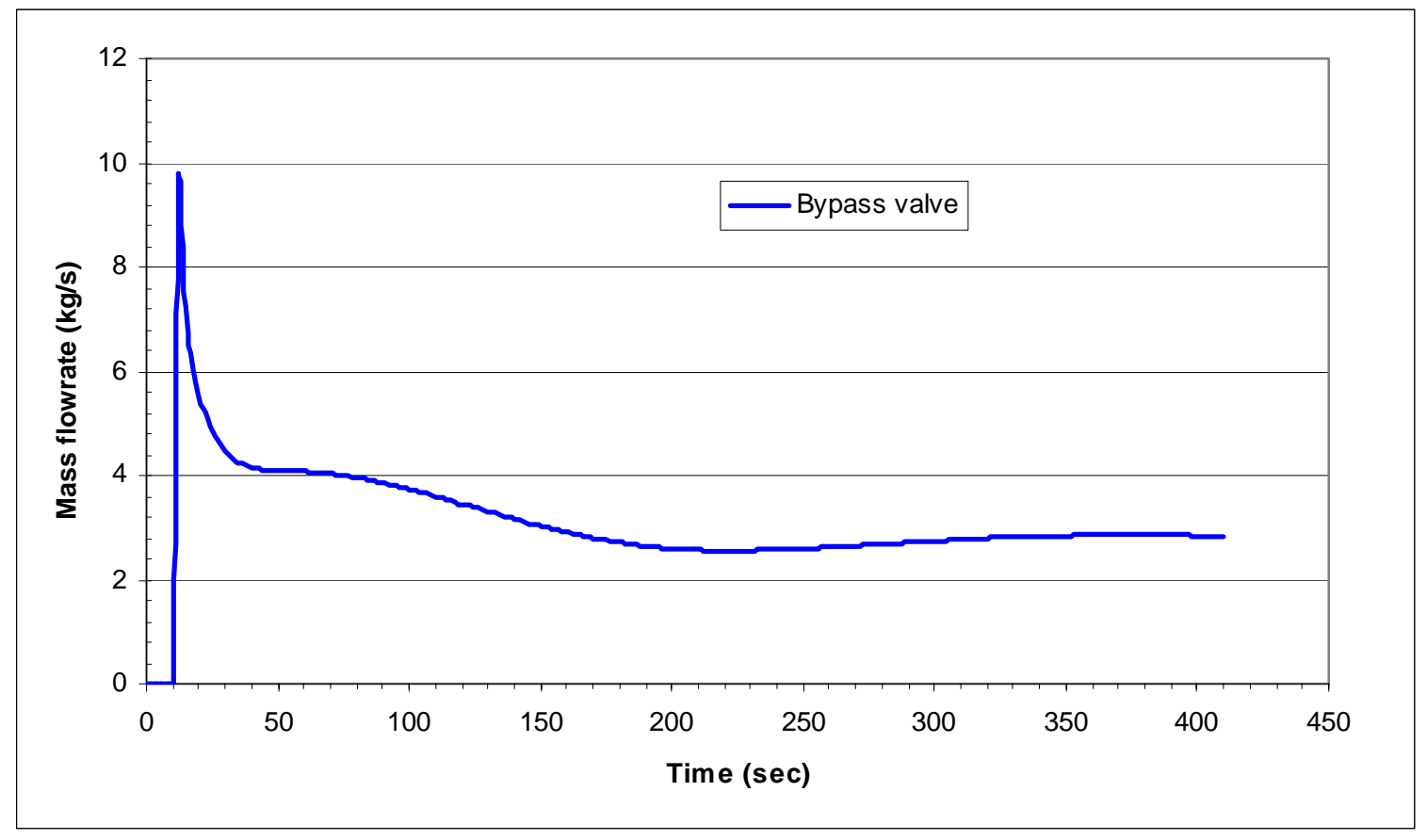

Figure 6.25 Bypass valve mass flowrate in 10\% load step reduction with bypass valve control used

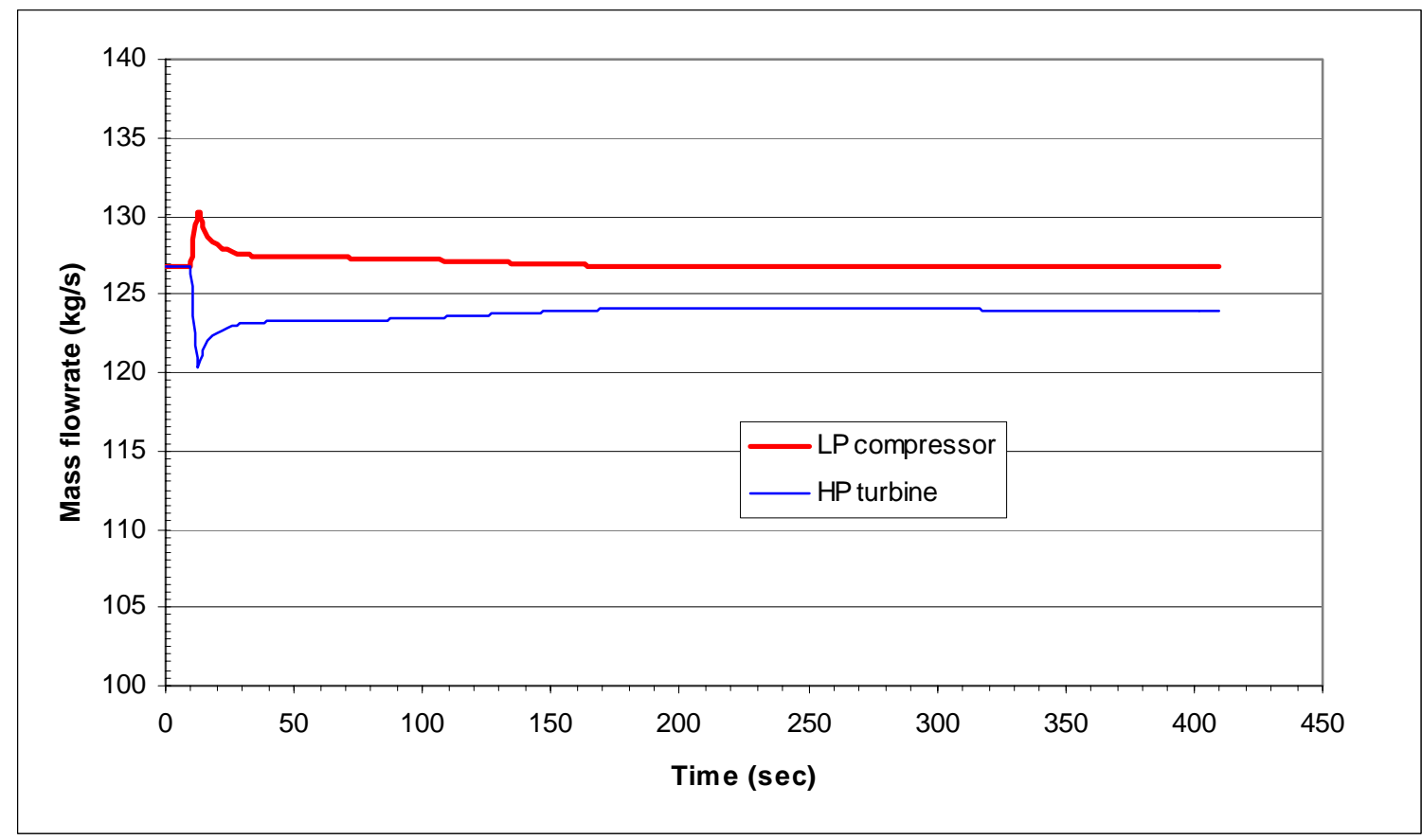

Figure 6.26 Mass flowrates of the HP turbine and LP compressor in 10\% load step reduction with bypass valve control 


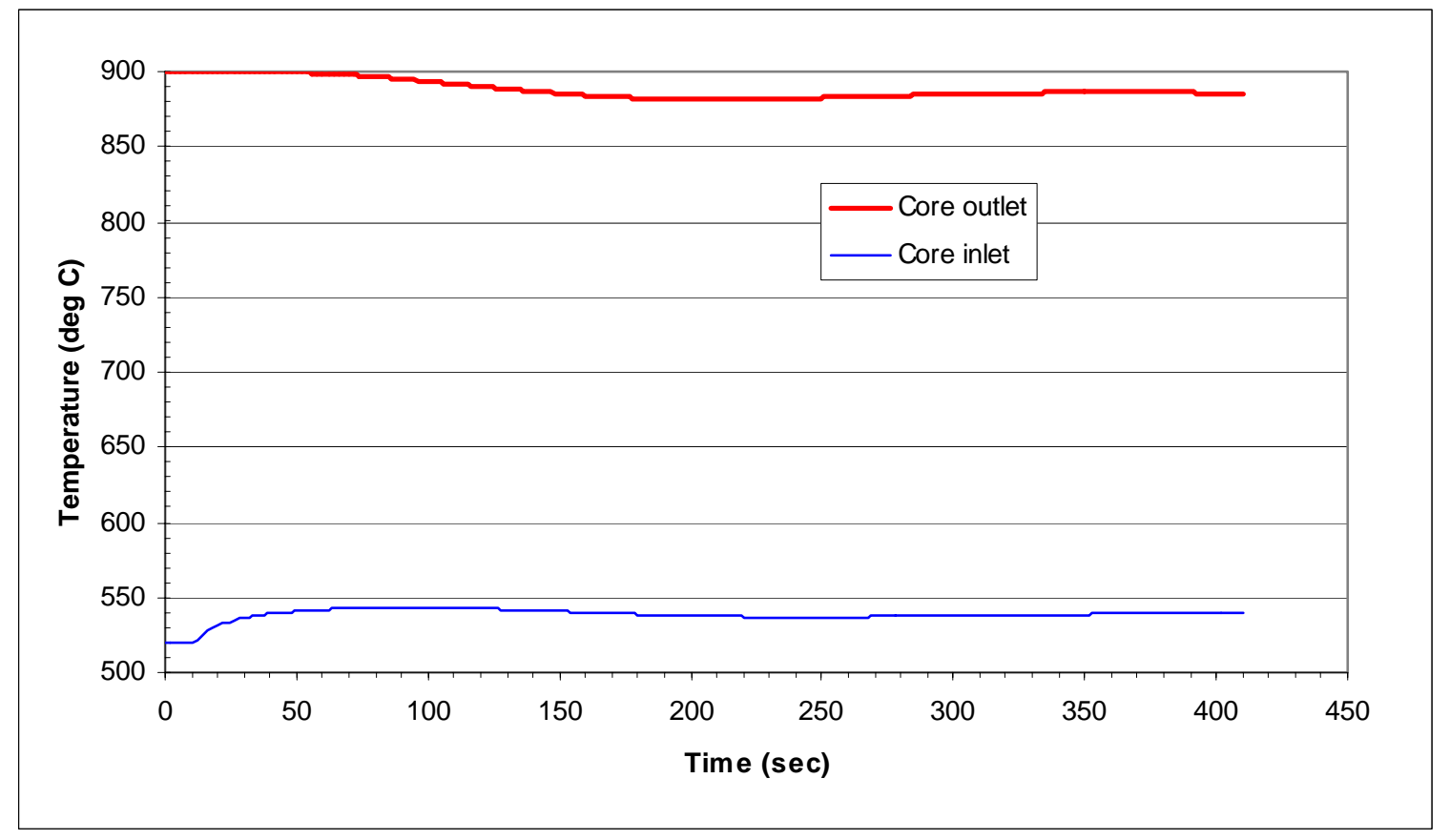

Figure 6.27 Core inlet and outlet temperatures in 10\% load step reduction with bypass valve control used

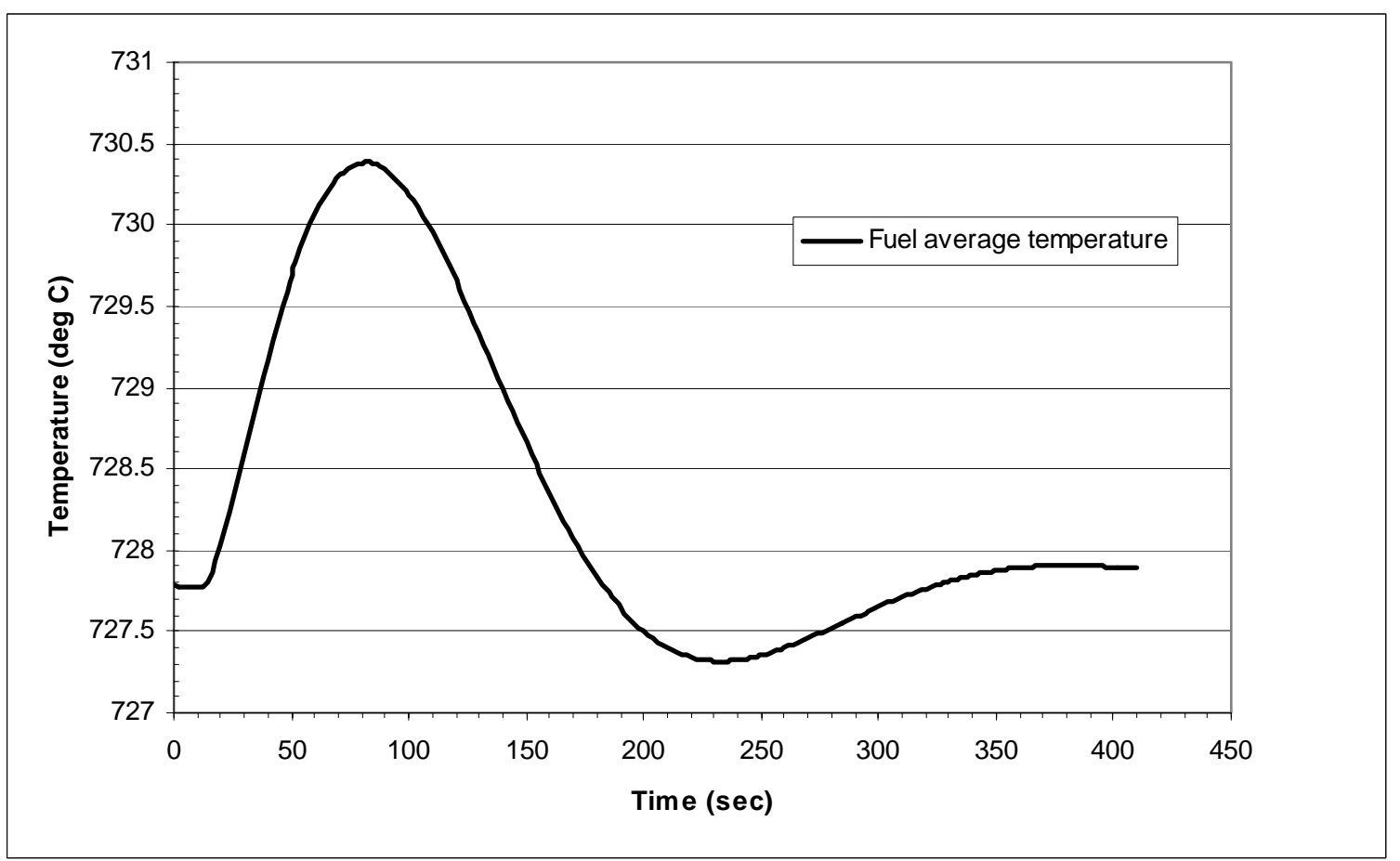

Figure 6.28 Fuel average temperature in $10 \%$ load step reduction with bypass valve control used 


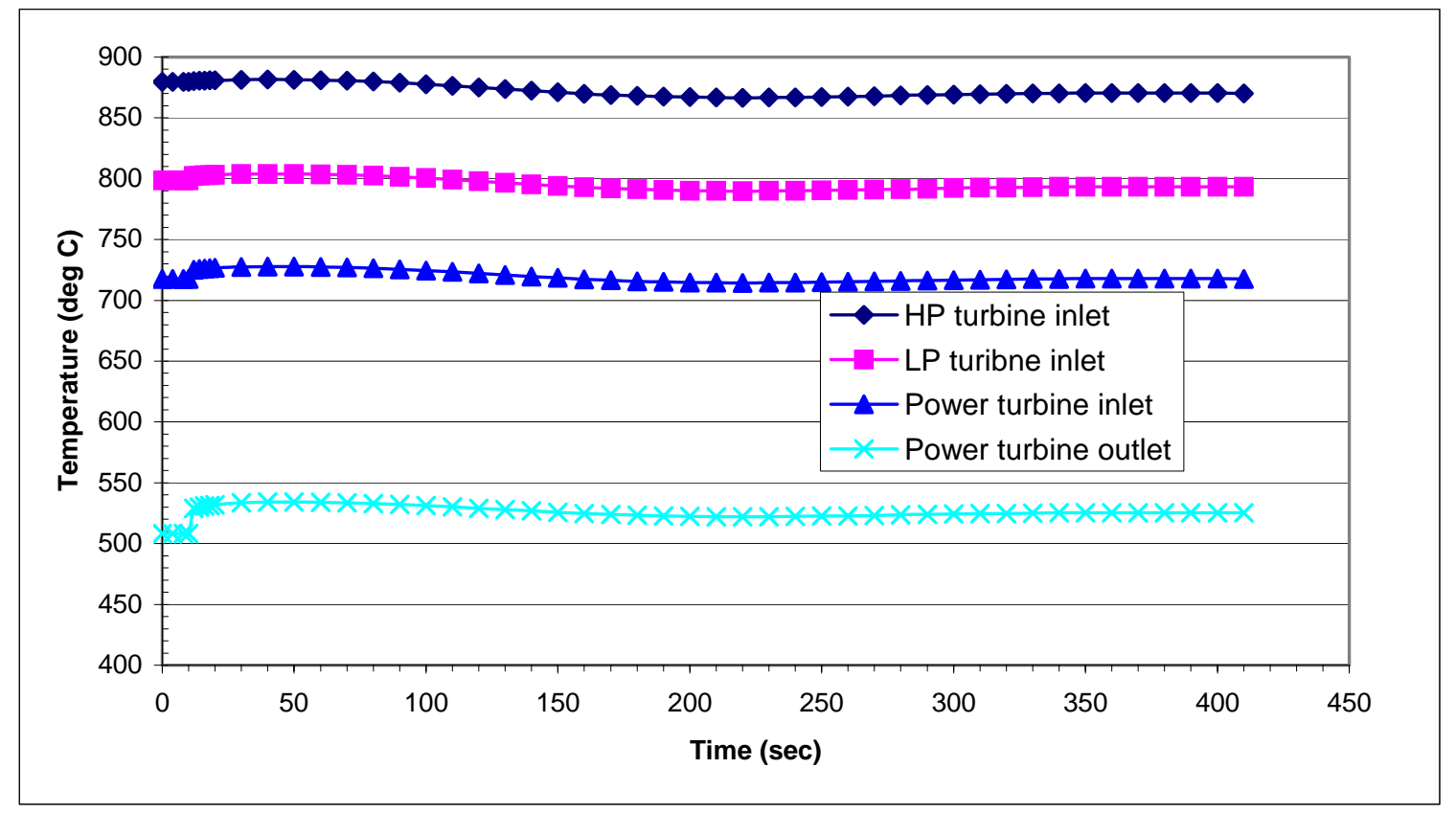

Figure 6.29 Temperatures of turbines in $10 \%$ load step reduction with bypass valve control used

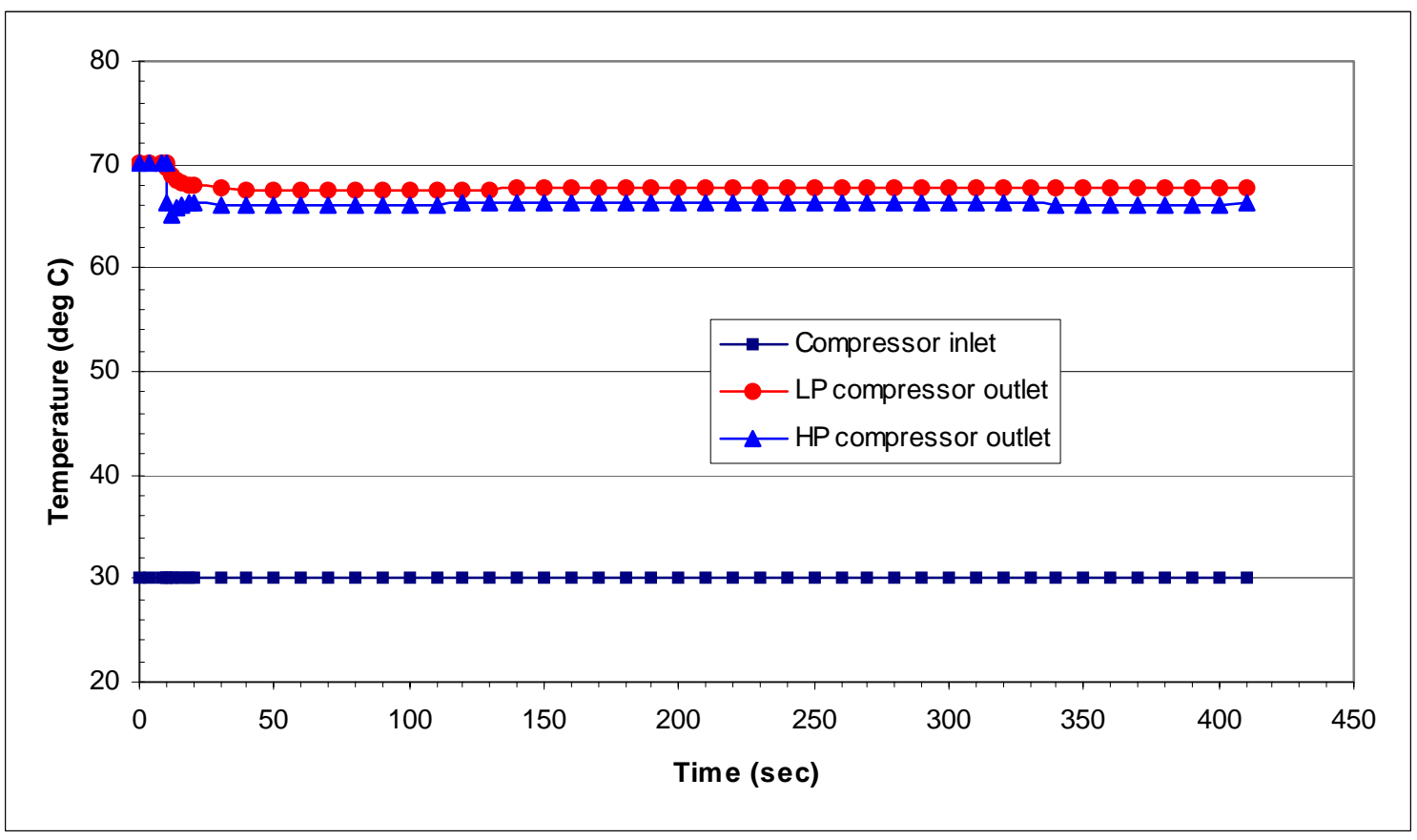

Figure 6.30 Temperatures of compressors in $10 \%$ load step reduction with bypass valve control used 


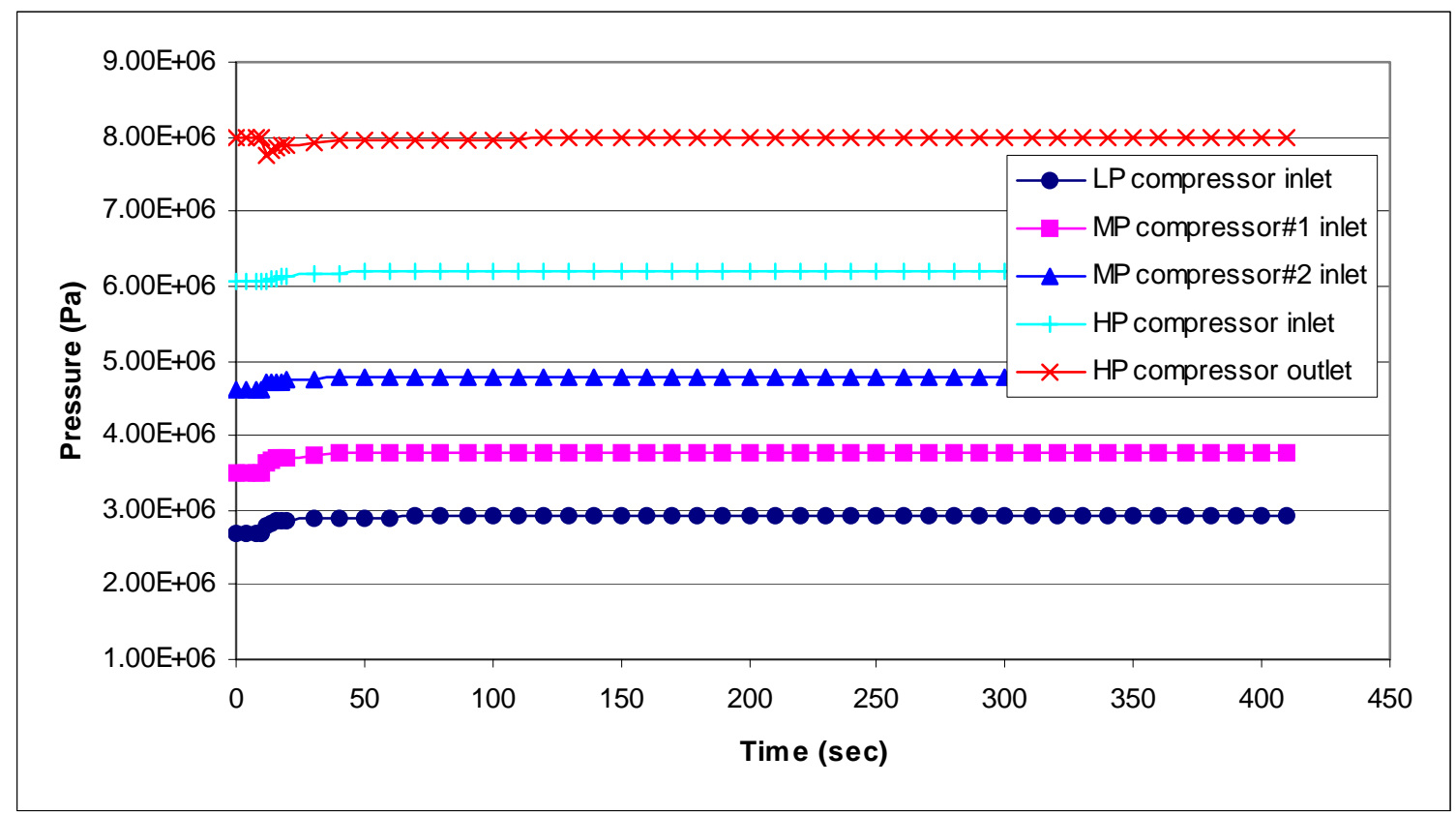

Figure 6.31 Pressures of compressors in 10\% load step reduction with bypass valve control used

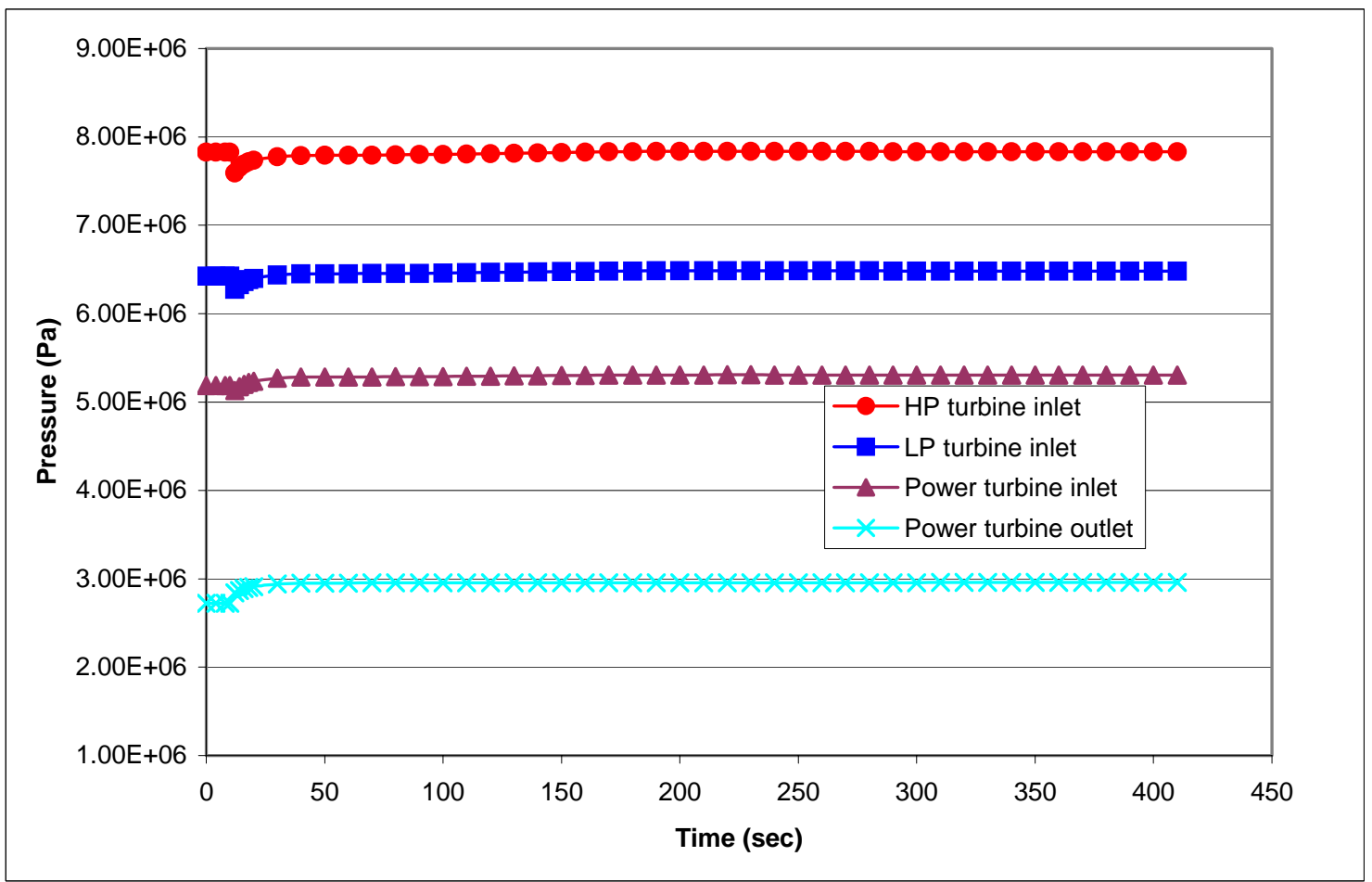

Figure 6.32 Pressures of turbines in 10\% load step reduction with bypass valve control used 


\subsection{2 $10 \% / \mathrm{min}$ and $5 \% / \mathrm{min}$ load $\mathrm{ramp}$}

Two load ramp scenarios are simulated. The first is the load decrease from $100 \%$ to $50 \%$ followed by an up load from $50 \%$ to $100 \%$. For this ramp, only bypass valve control is used. The second ramp is a load decrease from $100 \%$ to $50 \%$ followed by $50 \%$ partial load operation. With this partial load operation, both bypass valve control and inventory control are used.

\section{Load ramp: $100 \% \rightarrow 50 \%$ at a rate of $10 \% / \mathrm{min}$ and $50 \% \rightarrow 100 \%$ at a rate of $5 \% / \mathrm{min}$}

At 10 seconds, the load decreases from $100 \%$ at a rate of $10 \% / \mathrm{min}$. After reaching $50 \%$, the load remains at this level until 2000 seconds. At 2000 seconds, the load increases to $100 \%$ at a rate of $5 \% / \mathrm{min}$.

For simulating this load ramp, a centrifugal compressor map is used. The following control methods are used:

(1) Bypass valve control;

(2) Control rod control is used to maintain the core outlet temperature at $900^{\circ} \mathrm{C}$.

The plant responses are shown in Figures 6.33 to 6.39 .

In Figure 6.33, one can see that the reactor fission power follows the load ramp after about a 100 to 200 second delay. From the figures, when the fission power goes back to $100 \%$, the bypass valve is almost closed. And all the temperatures and pressures in the cycle resume their original values.

During the period of time from 310 seconds to 2000 seconds for $50 \%$ partial load operation, the bypass valve mass flowrate is about $19 \mathrm{~kg} / \mathrm{s}$. The fission power level is $55 \%$. This shows that the bypass valve control does not significantly degrade the cycle efficiency. However, the core inlet temperature rises $170^{\circ} \mathrm{C}$, which will violate temperature limits. This introduces the consideration of using inventory control to eliminate the large temperature increase and the thermal stress on the components. 


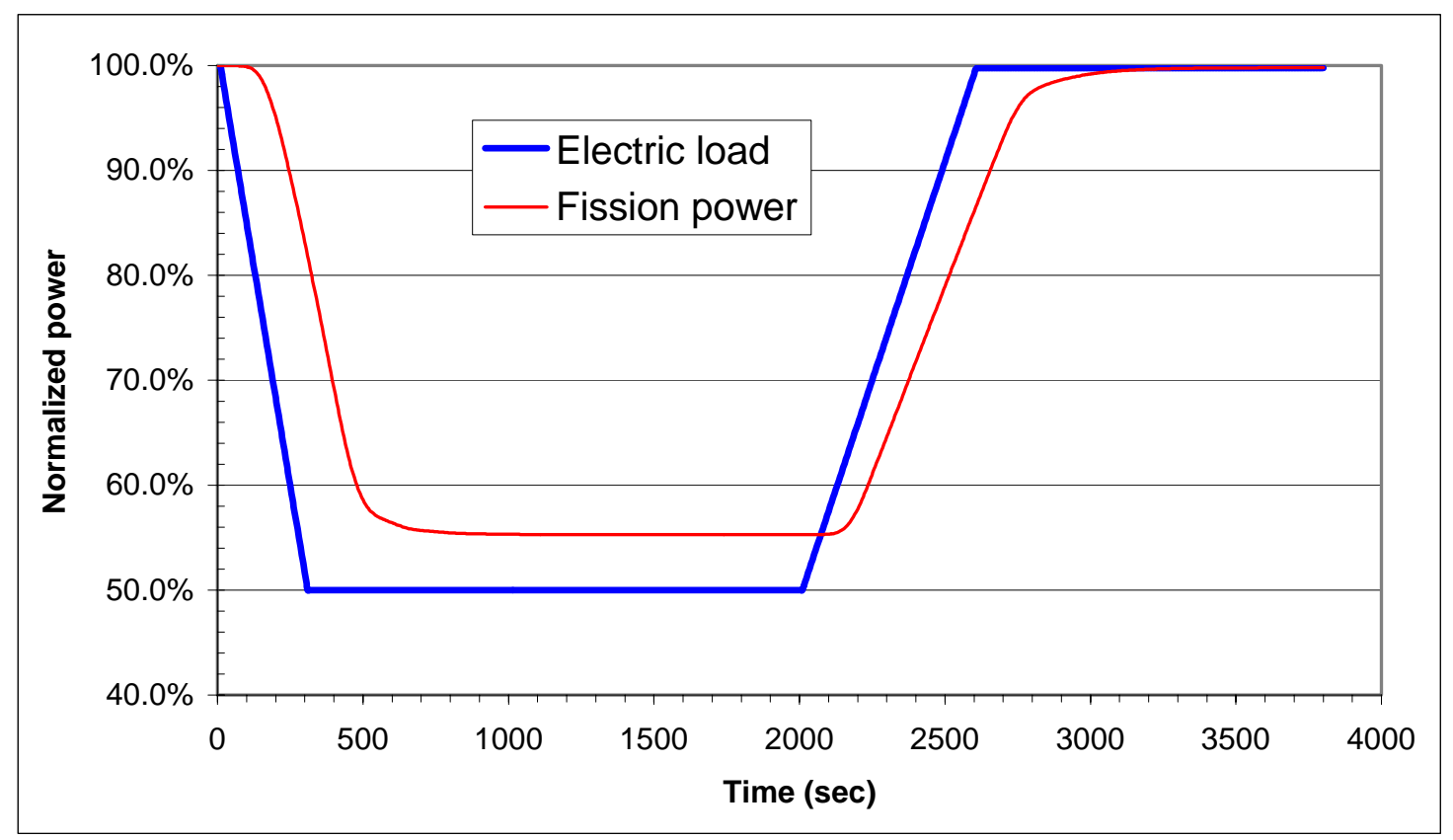

Figure 6.33 Electric load and reactor fission power in the load ramp from $100 \%$ to $50 \%$ at $10 \% / \mathrm{min}$ and then back up to $100 \%$ at $5 \% / \mathrm{min}$, bypass valve control is used and centrifugal compressor map is used

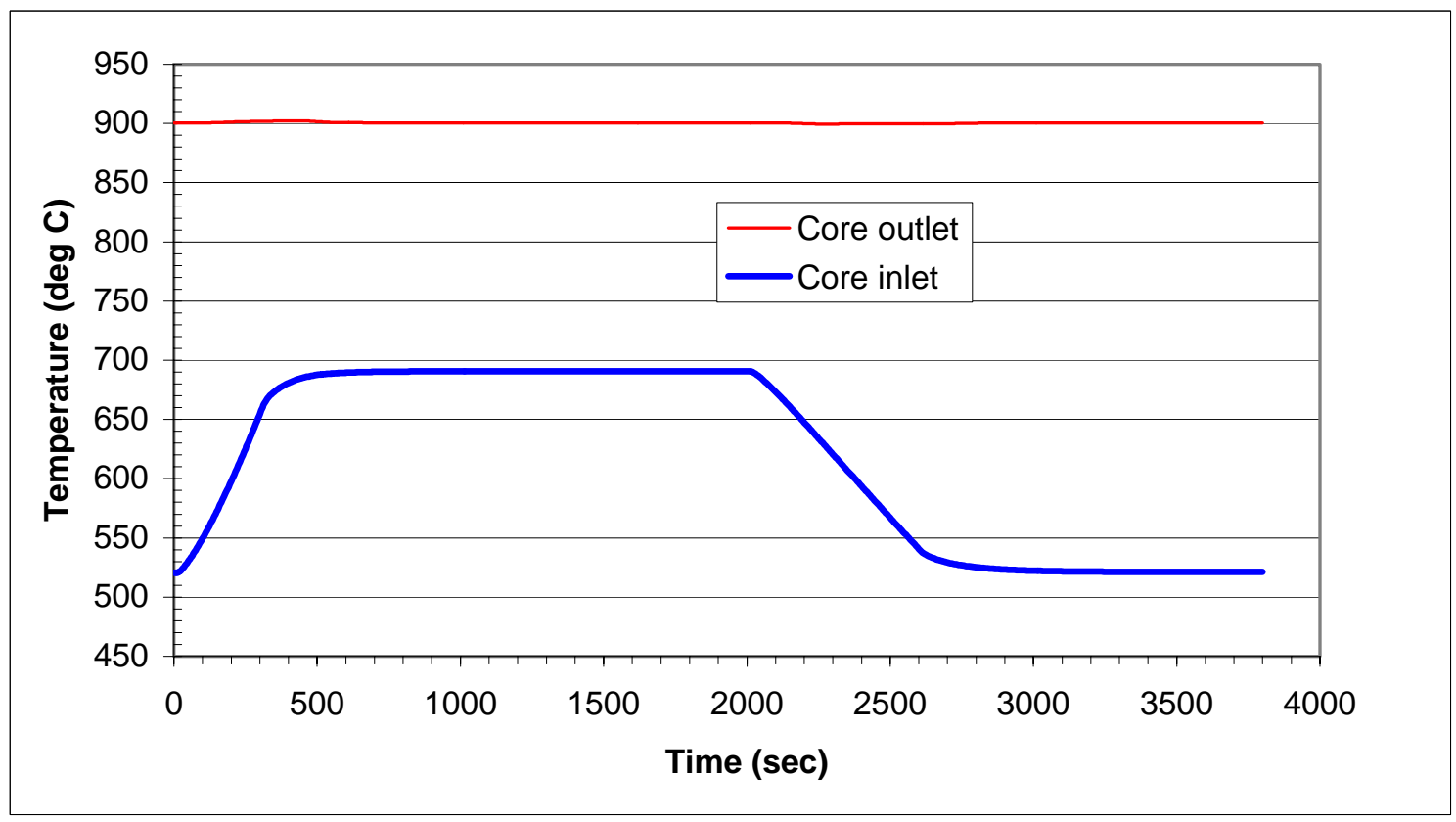

Figure 6.34 Core outlet/inlet temperature in the load ramp from $100 \%$ to $50 \%$ at $10 \% / \mathrm{min}$ and then back up to $100 \%$ at $5 \% / \mathrm{min}$, bypass valve control is used and centrifugal compressor map is used 


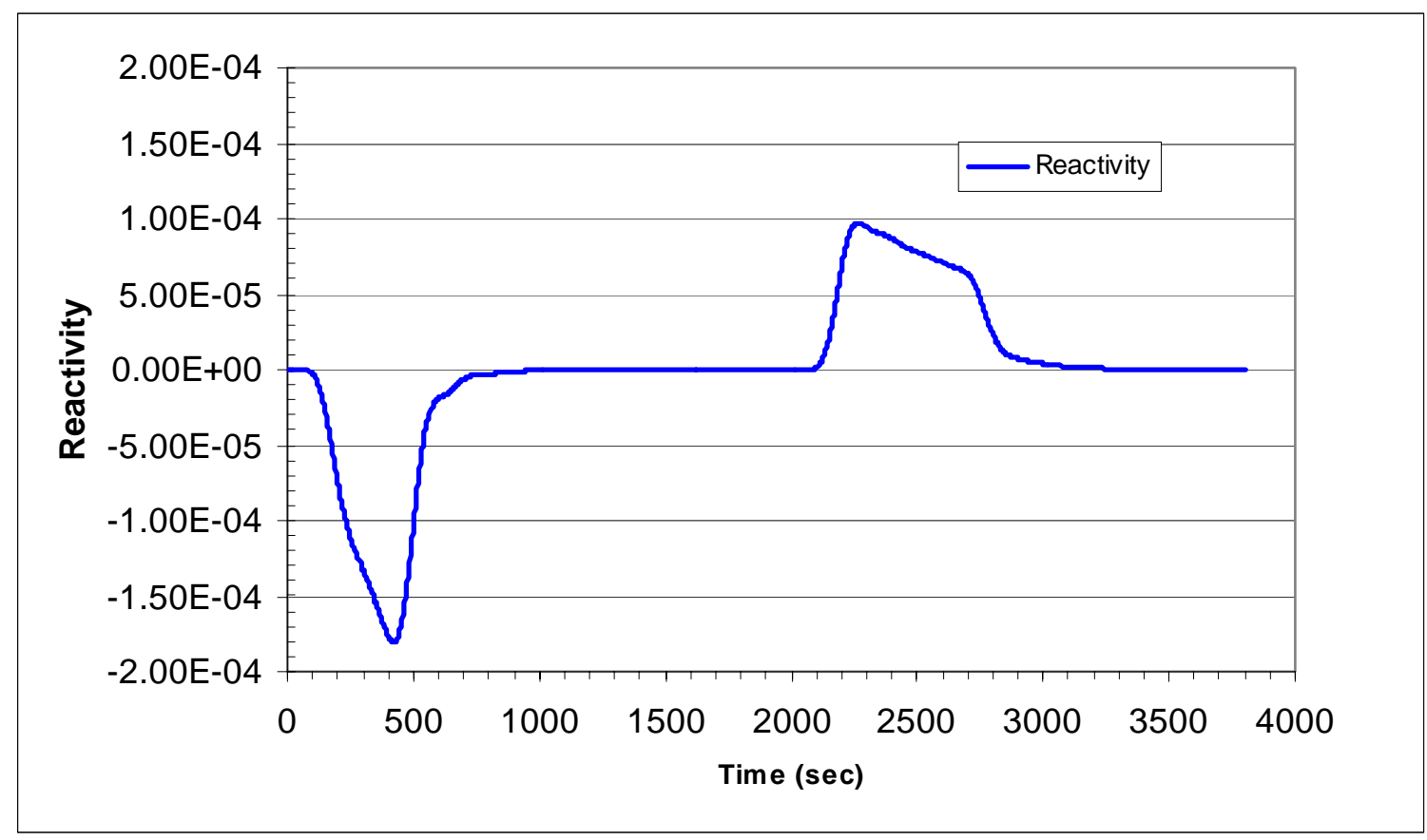

Figure 6.35 Reactivity in the load ramp from $100 \%$ to $50 \%$ at $10 \% / \mathrm{min}$ and then back up to $100 \%$ at $5 \% / \mathrm{min}$, bypass valve control is used and centrifugal compressor map is used

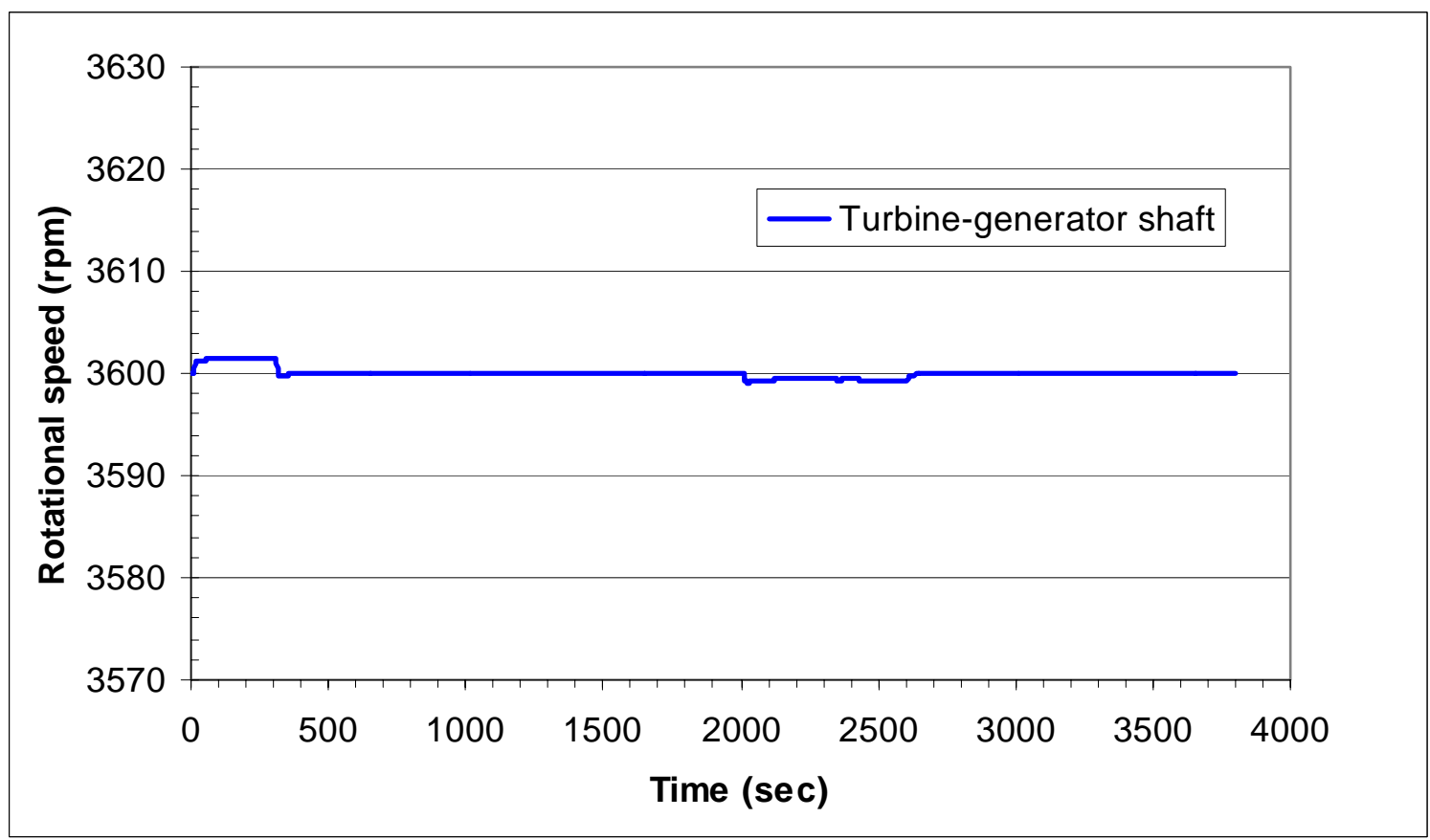

Figure 6.36 Turbine-generator shaft speed in the load ramp from $100 \%$ to $50 \%$ at $10 \% / \mathrm{min}$ and then back up to $100 \%$ at $5 \% / \mathrm{min}$, bypass valve control is used centrifugal compressor map is used 


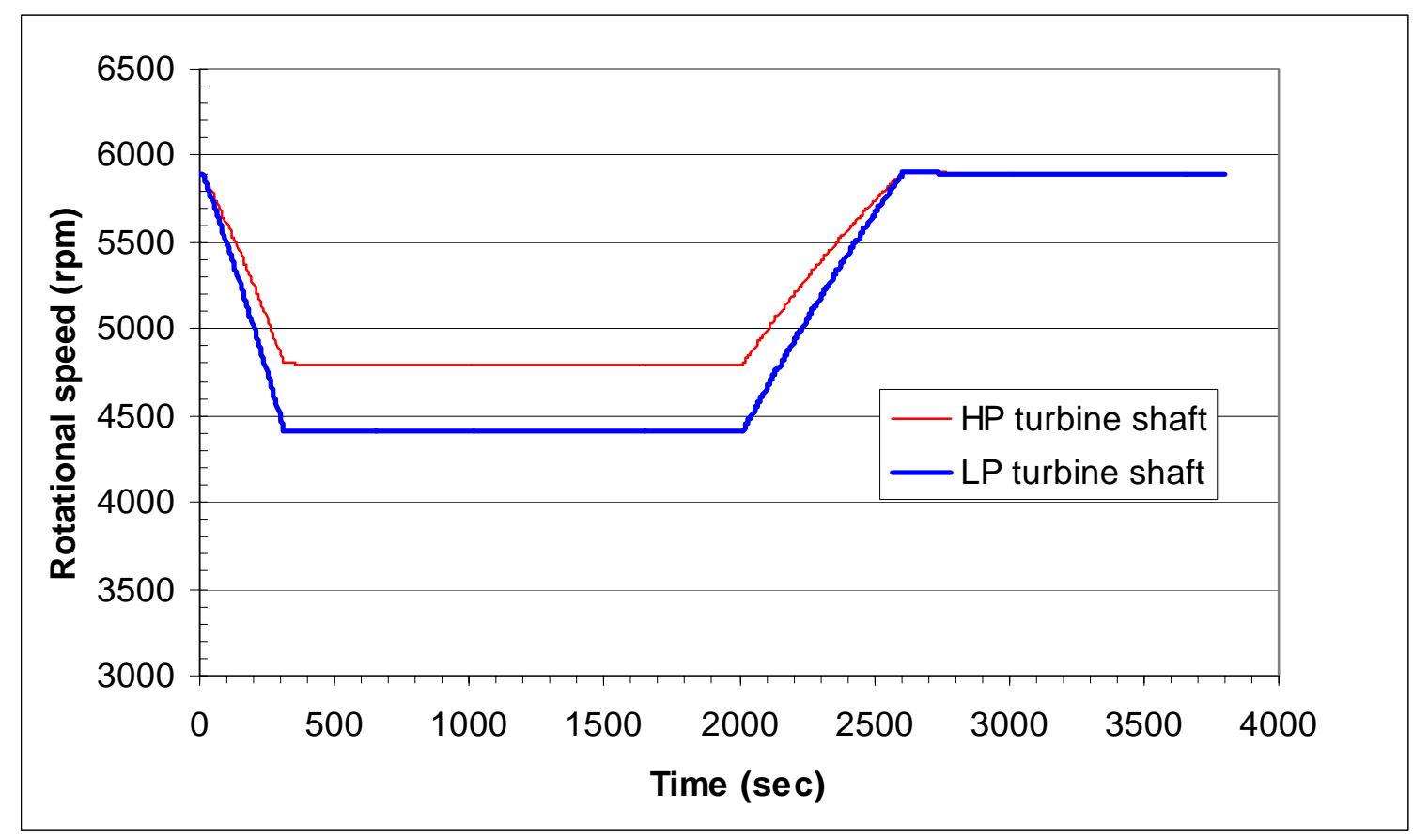

Figure 6.37 HP turbine shaft speed and LP turbine shaft speed in the load ramp from 100\% to $50 \%$ at $10 \% / \mathrm{min}$ and then back up to $100 \%$ at $5 \% / \mathrm{min}$, bypass valve control is used centrifugal compressor map is used

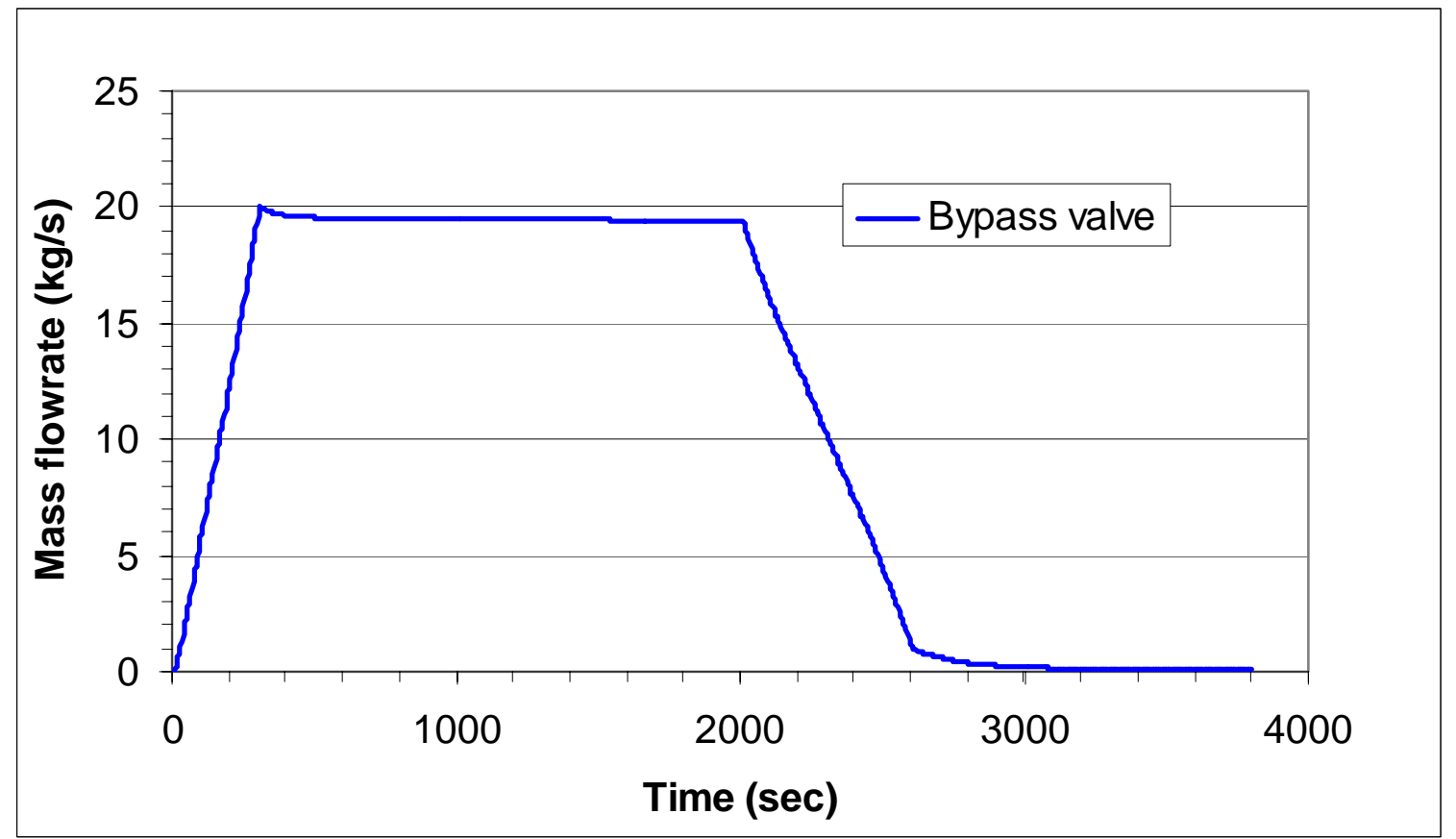

Figure 6.38 Bypass valve mass flowrate in the load ramp from $100 \%$ to $50 \%$ at $10 \% / \mathrm{min}$ and then back up to $100 \%$ at $5 \% / \mathrm{min}$, bypass valve control is used and centrifugal compressor map is used 


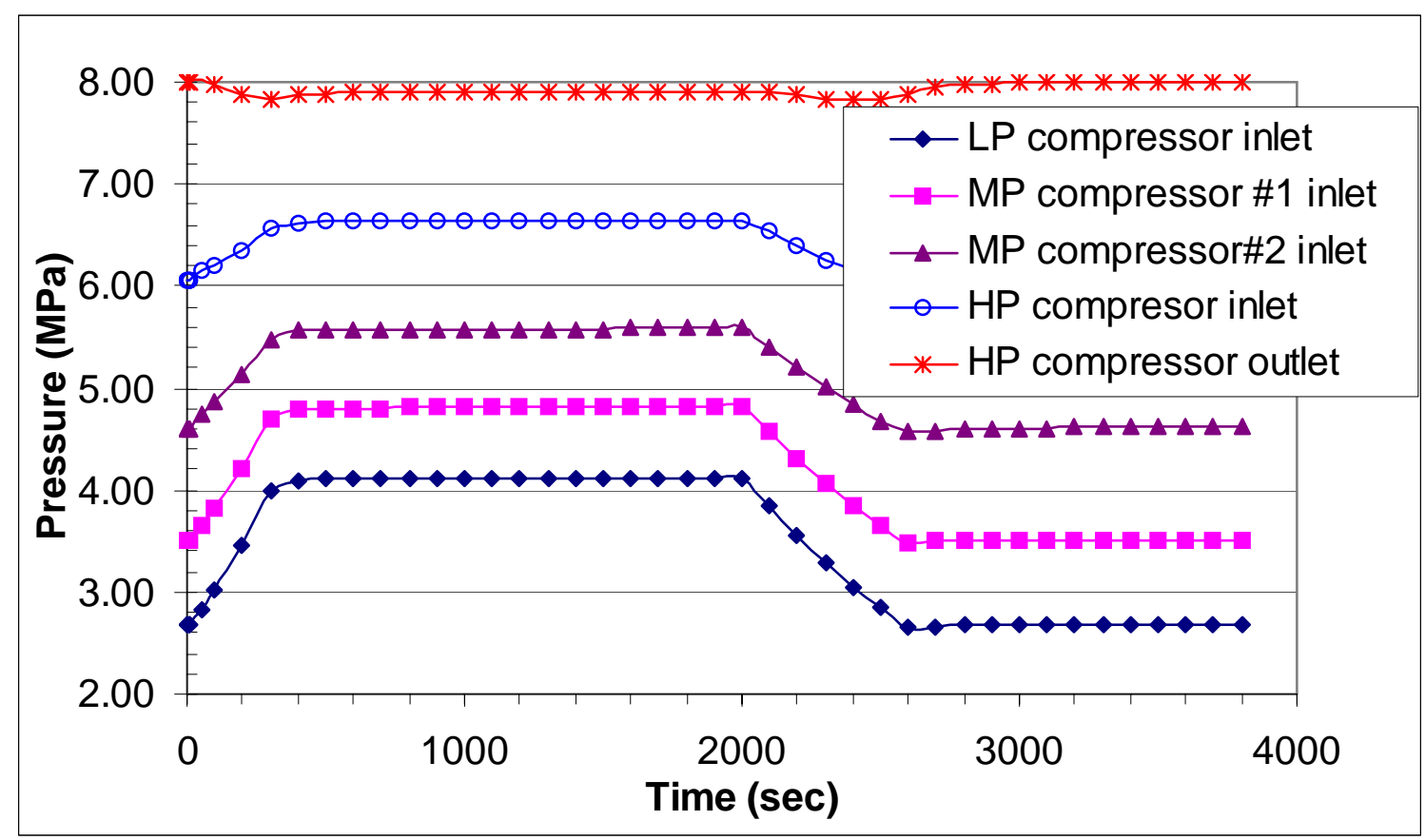

Figure 6.39 Compressor pressures in the load ramp from $100 \%$ to $50 \%$ at $10 \% / \mathrm{min}$ and then back up to $100 \%$ at $5 \% / \mathrm{min}$, bypass valve control is used and centrifugal compressor map is used

\section{Load ramp from $100 \%$ to $50 \%$ with a rate of $10 \% / \mathrm{min}$}

This transient simulates a load ramp from $100 \%$ to $50 \%$ with a rate of $10 \% / \mathrm{min}$. The axial compressor map is used. The following control actions are employed:

(1) Bypass valve control is used to maintain the turbine-generator shaft speed;

(2) Inventory control is used to adjust the inventory of the PCU;

(3) The primary mass flowrate is set identical to the secondary mass flowrate.

In the primary system, control rod control is not used so that the reactor fission power is adjusted to solely depend on the temperature coefficient of reactivity. The helium inlet temperature of the compressors is kept constant at $30^{\circ} \mathrm{C}$. Figures 6.40 to 6.50 show the responses of the plant.

At 10 seconds, the load starts to decrease with a rate of $10 \% / \mathrm{min}$, the bypass valve opens instantaneously and the inventory valve also opens to withdraw helium from the PCU to the inventory vessel, as shown in Figures 6.40 and 6.43. When the bypass valve opens, the HP turbine shaft speed and LP turbine shaft speed decrease until the load reaches $50 \%$ at 310 seconds. The core inlet temperature increases prior to 310 seconds. After 310 seconds, the load remains at $50 \%$ of the full load. However, the inventory valve is still opening, the helium inventory decreases and mass flowrate in the PCU decreases continuously. The HP turbine shaft speed and LP turbine shaft speed increase and the core inlet temperature decreases. At about 1290 seconds, the inventory valve closes. The plant reaches a new steady state $-50 \%$ partial load operation. 
At 50\% partial load operation, the bypass valve is "feathered" - with bypass valve mass flowrate about $0.8 \mathrm{~kg} / \mathrm{s}$. The LP compressor mass and power turbine mass flowrate are about $63.4 \mathrm{~kg} / \mathrm{s}$ and $62.6 \mathrm{~kg} / \mathrm{s}$, respectively. The core inlet temperature is $520.8^{\circ} \mathrm{C}$ and the core outlet temperature is $902.2^{\circ} \mathrm{C}$, a little higher than the nominal value of $900^{\circ} \mathrm{C}$. The pressure in the high-pressure zone is $4.0 \mathrm{MPa}$, and the pressure in the low-pressure zone is $1.32 \mathrm{MPa}$. The pressure ratio is very close to the original pressure ratio. At the new steady state, the HP turbine shaft speed and LP turbine shaft speed are $7998 \mathrm{rpm}$ and $8046 \mathrm{rpm}$, respectively. The fission power is $124.0 \mathrm{MW}$ th. The cycle efficiency is even slightly higher than that of full power operation. At $50 \%$ load, the mass flow reduces to approximately half of the nominal value through the IHX and recuperator, while the volume and heat transfer area remain fixed. The IHX and recuperator effectiveness increase from $95 \%$ to $95.6 \%$. The increasing effectiveness of heat exchangers at partial power agrees with the literature[1]. This contributes in part to the slightly increasing cycle efficiency at partial load operation.

The significant benefit of using inventory control is that it allows the cycle to operate at partial load with temperatures roughly the same as the nominal values.

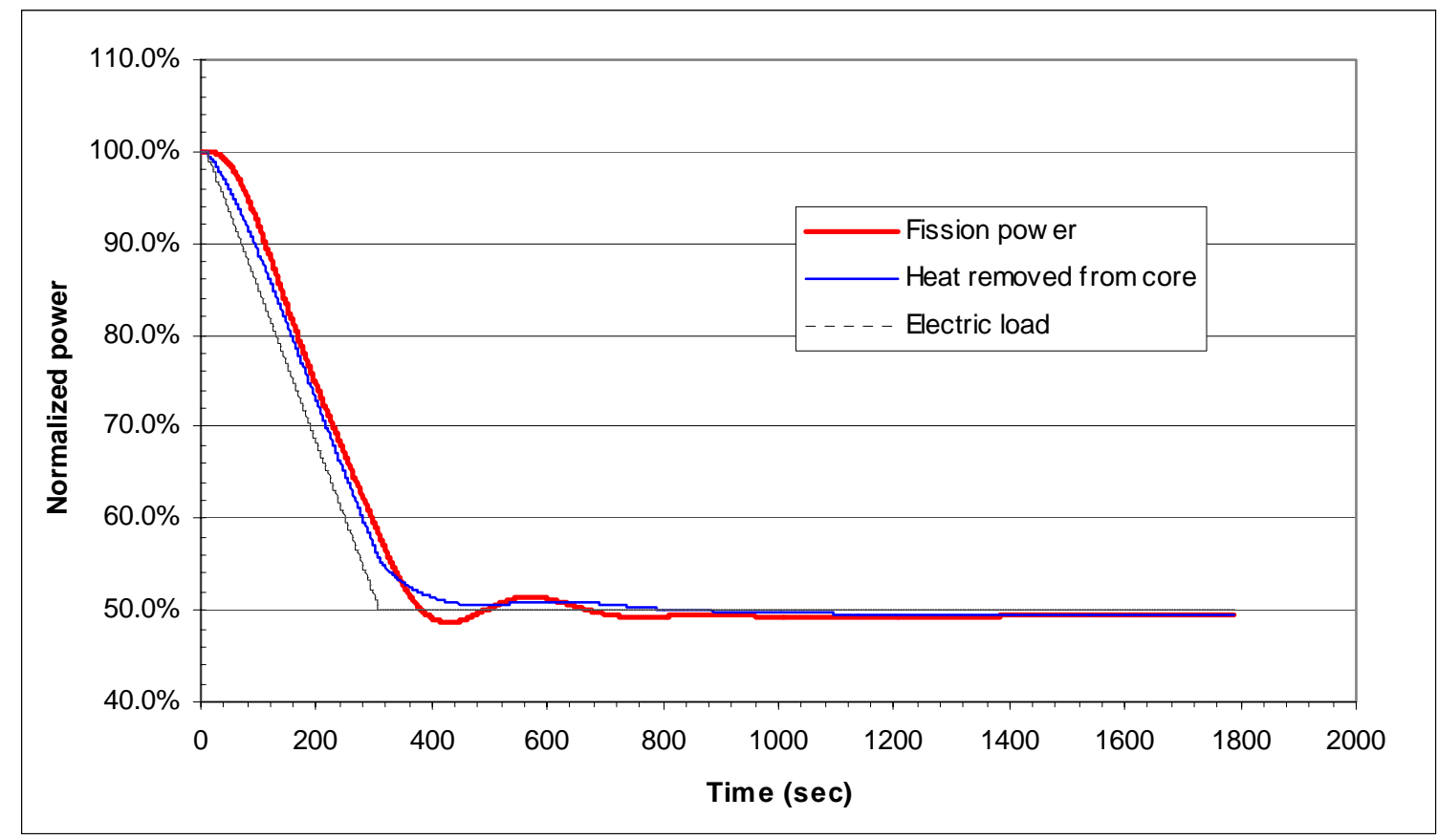

Figure 6.40 Electric load and reactor fission power in a load ramp from $100 \%$ to $50 \%$ at a rate of $10 \% / \mathrm{min}$, both bypass control and inventory control are used 


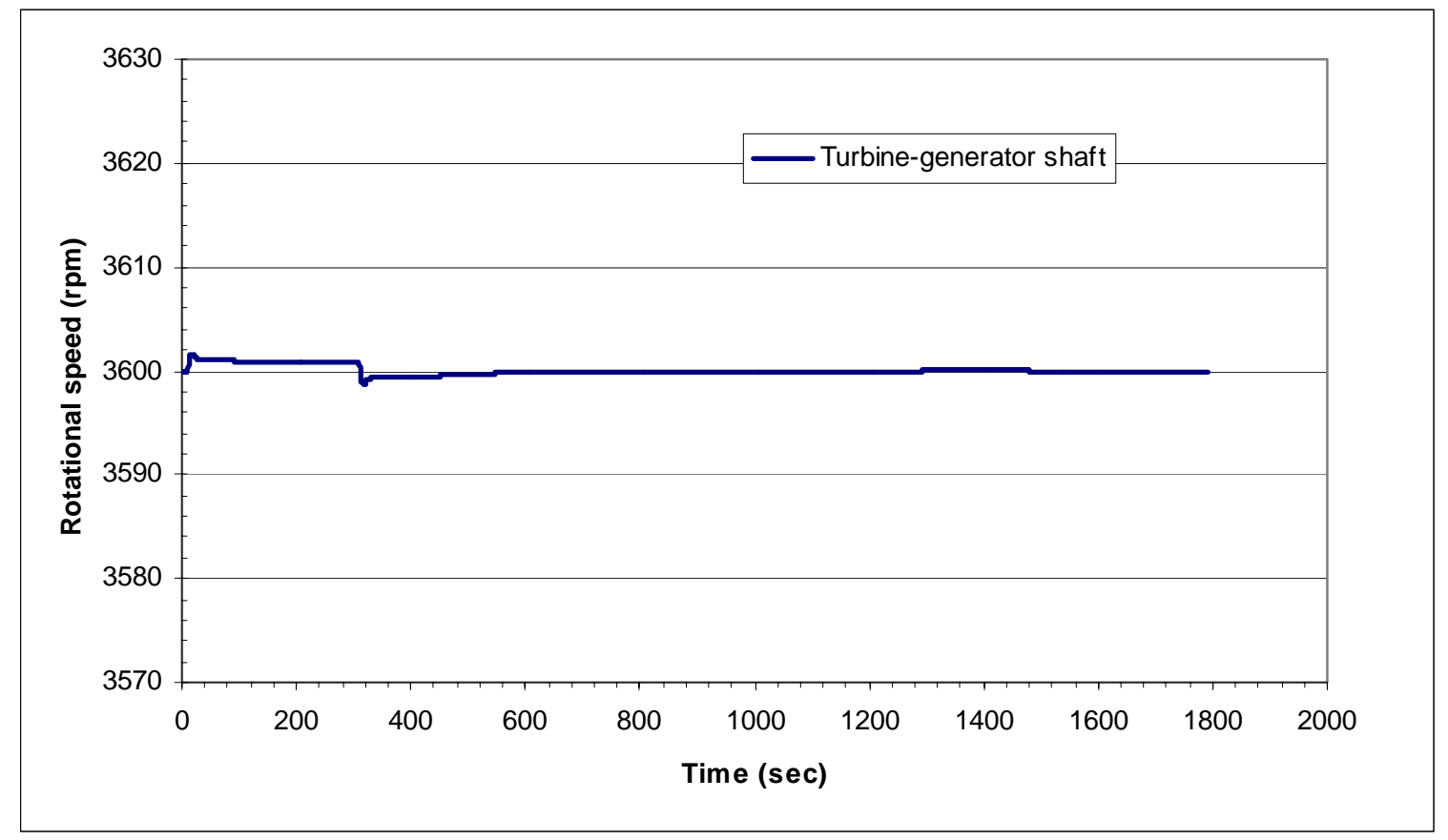

Figure 6.41 Turbine-generator shaft speed in a load ramp from $100 \%$ to $50 \%$ at a rate of $10 \% / \mathrm{min}$, both bypass valve control and inventory control are used

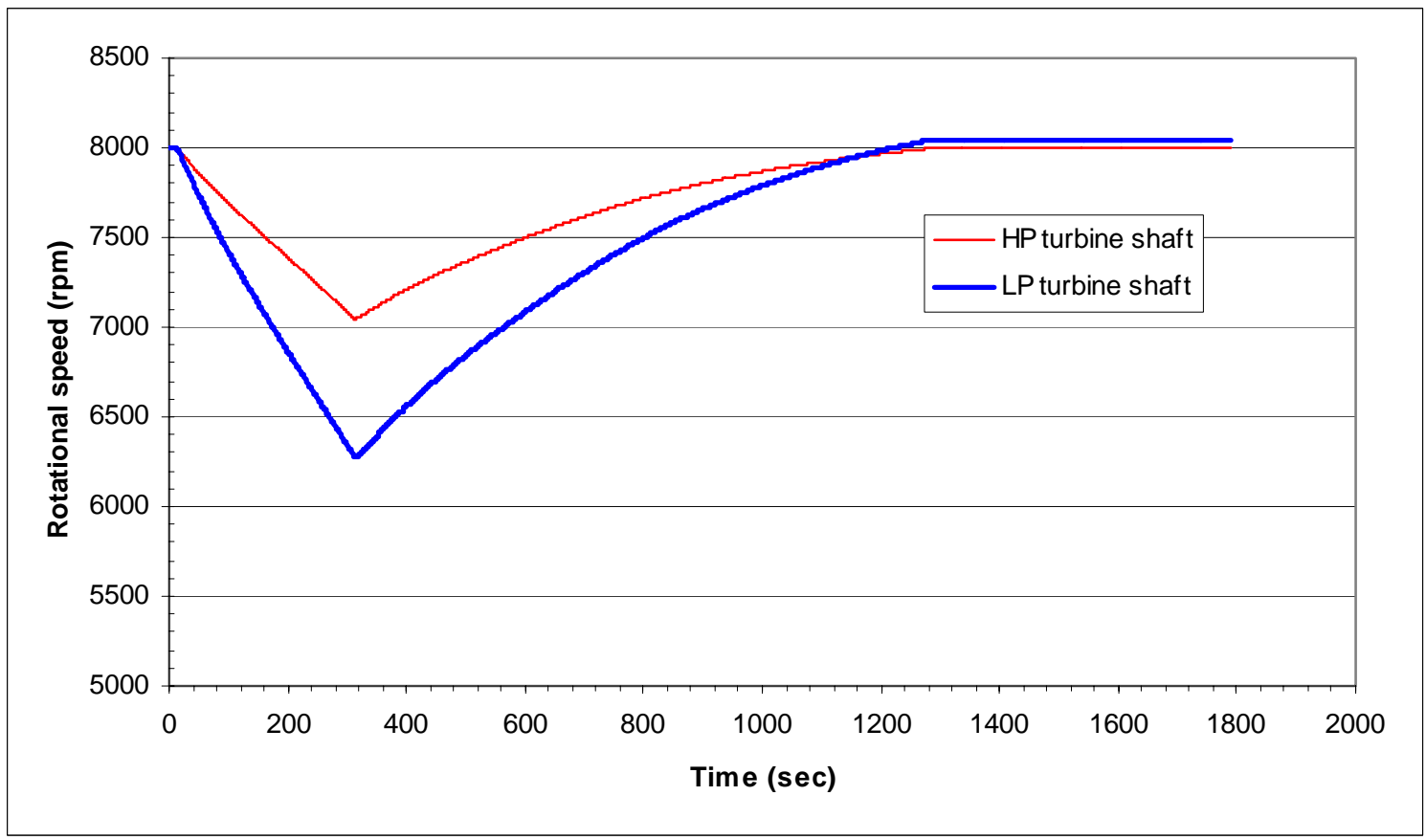

Figure 6.42 HP turbine shaft speed and LP turbine shaft speed in a load ramp from $100 \%$ to $50 \%$ at a rate of $10 \% / \mathrm{min}$, both bypass valve control and inventory control are used 


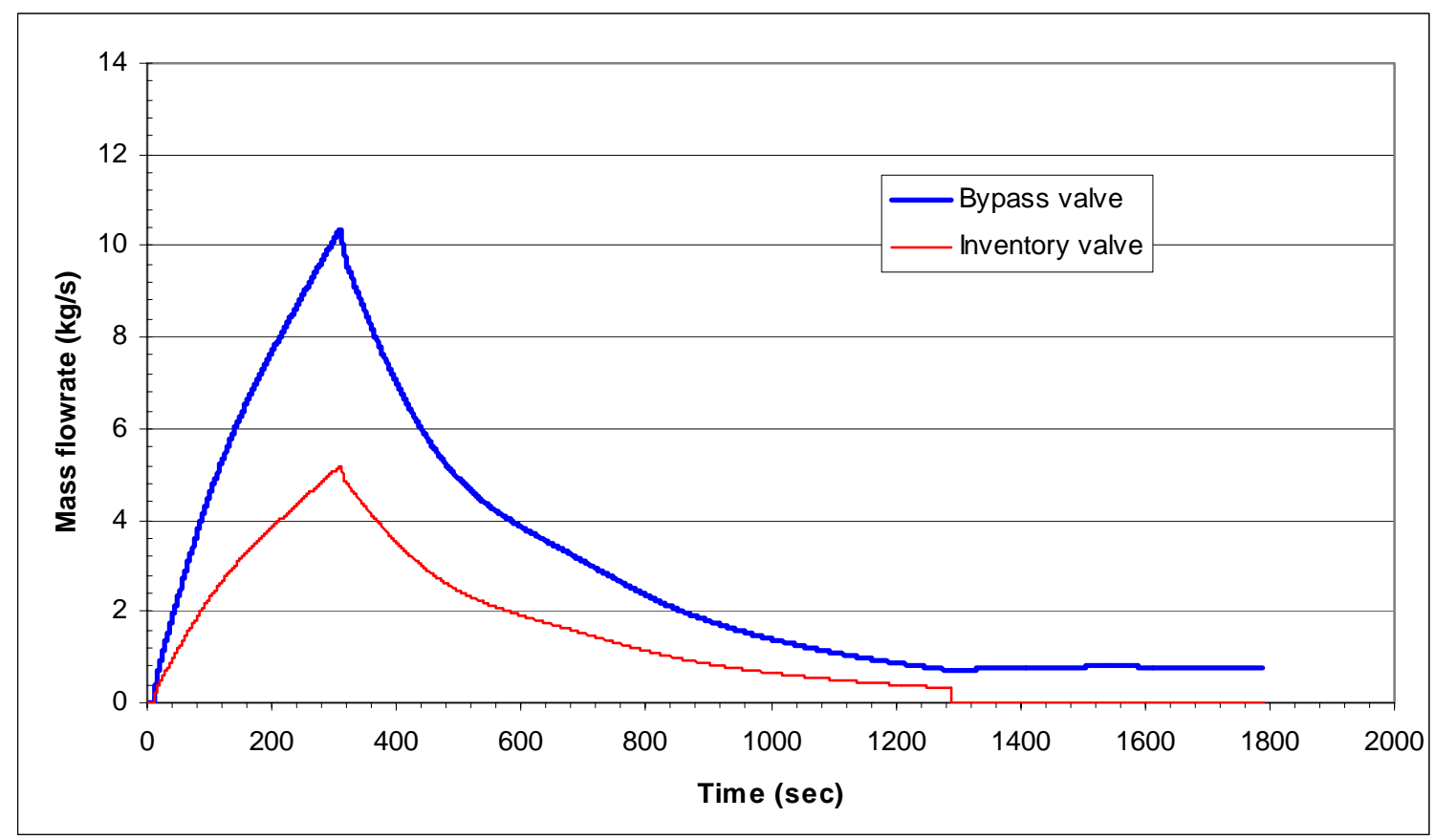

Figure 6.43 Bypass valve and inventory valve mass flowrate in a load ramp from $100 \%$ to $50 \%$ at a rate of $10 \% / \mathrm{min}$, both bypass valve control and inventory control are used

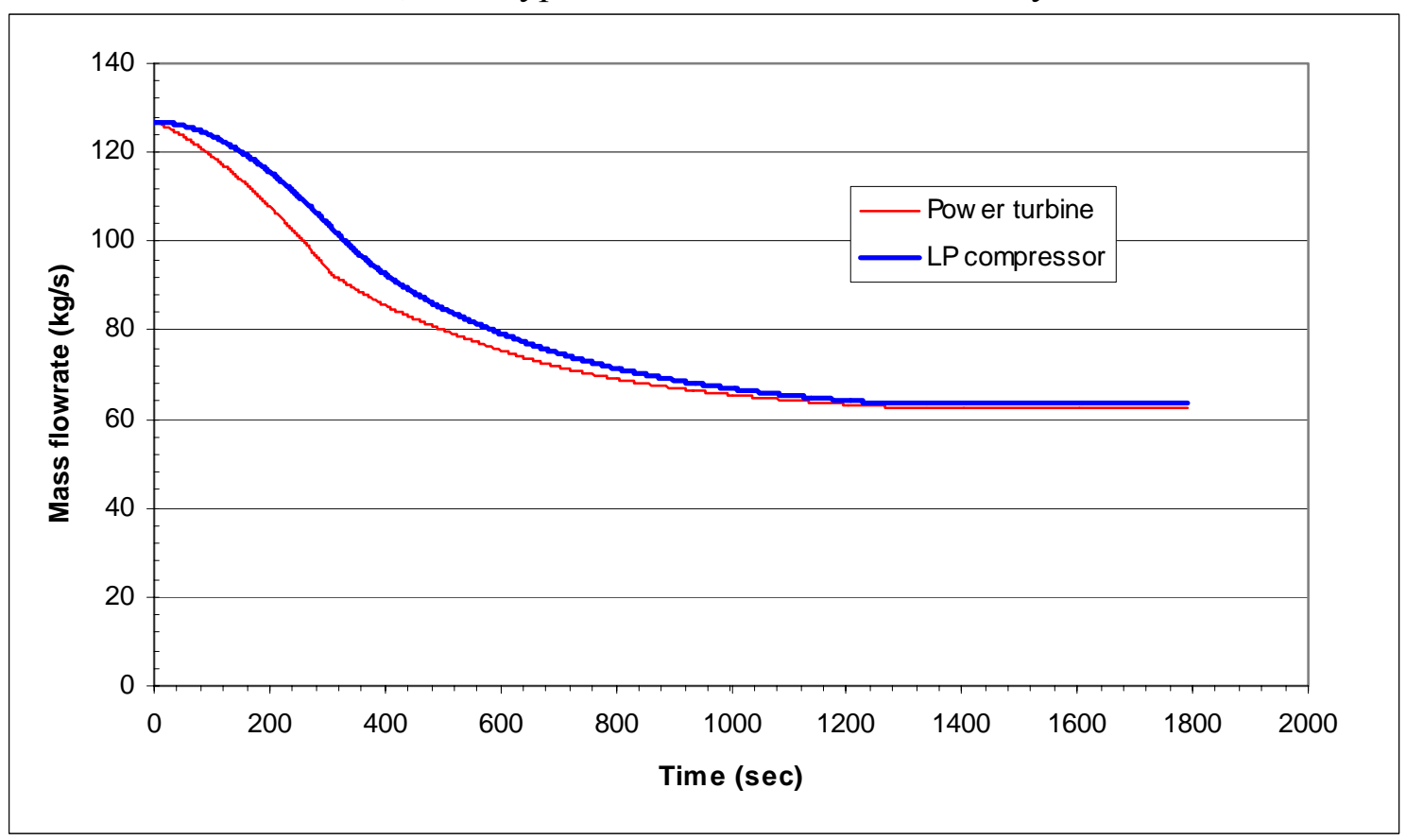

Figure 6.44 Power turbine and LP compressor mass flowrate in a load ramp from $100 \%$ to $50 \%$ at a rate of $10 \% / \mathrm{min}$, both bypass valve control and inventory control are used 


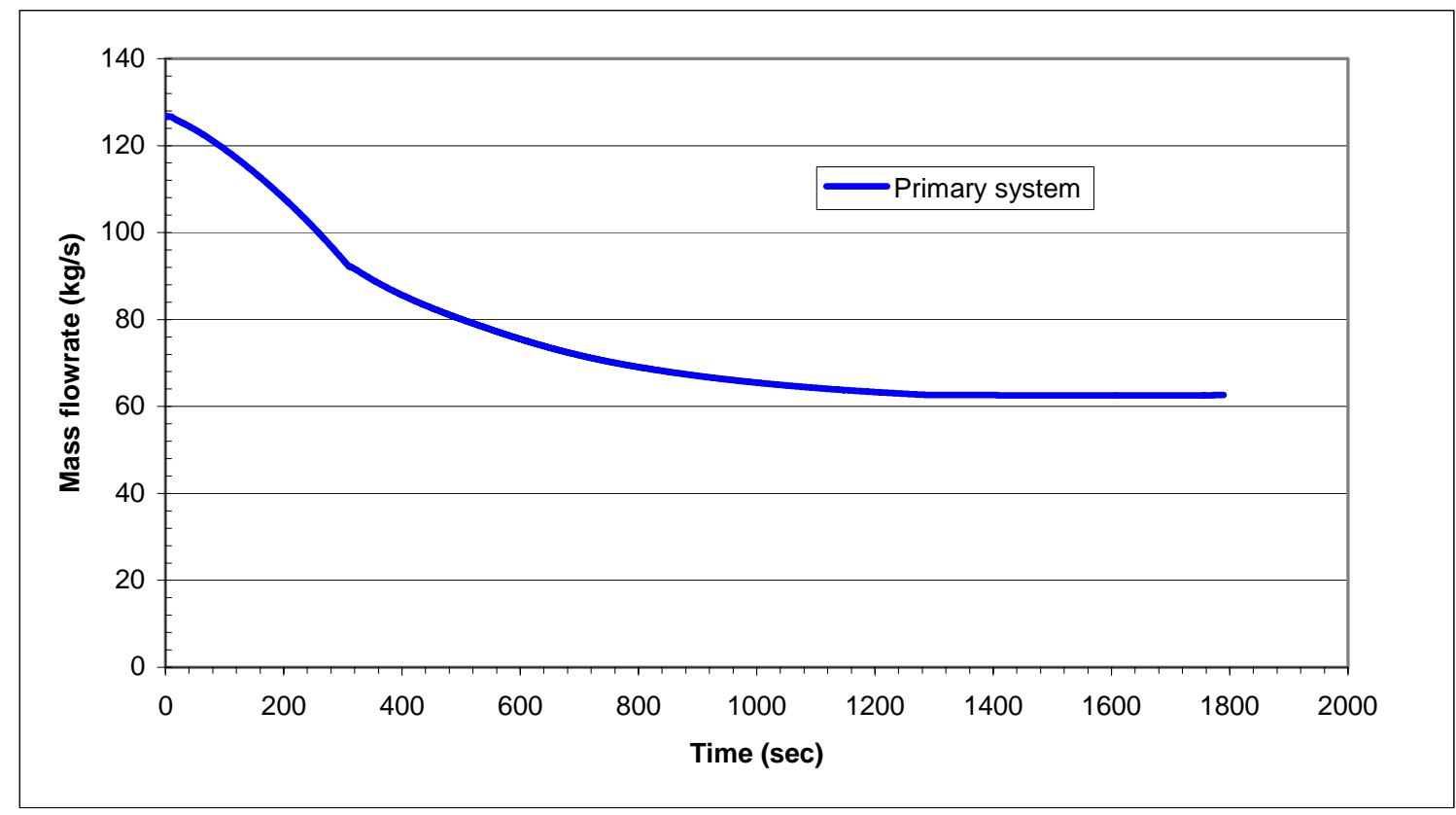

Figure 6.45 Primary system mass flowrate in a load ramp from $100 \%$ to $50 \%$ at a rate of $10 \% / \mathrm{min}$, both bypass valve control and inventory control are used

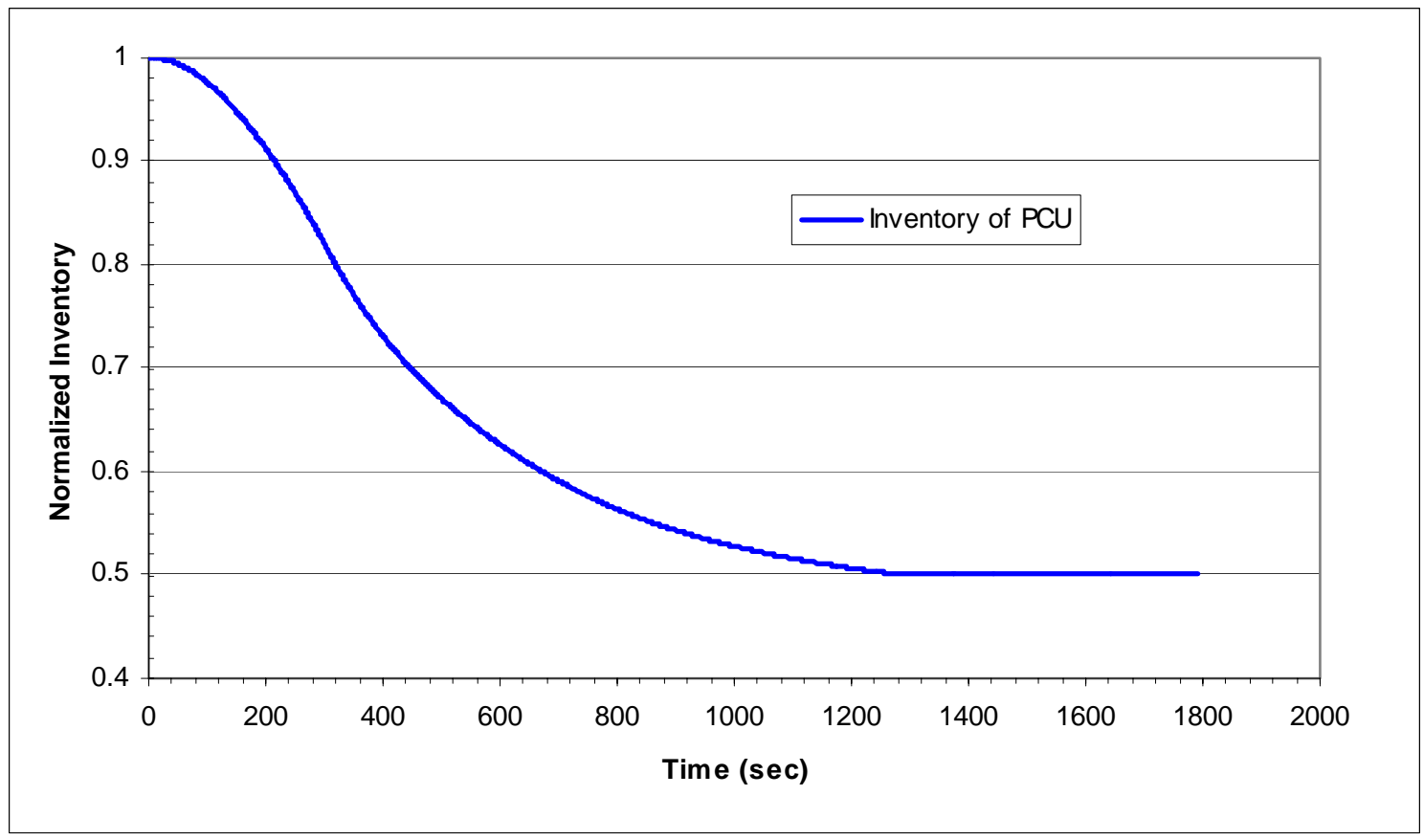

Figure 6.46 Helium inventory in the PCU in a load ramp from $100 \%$ to $50 \%$ at a rate of $10 \% / \mathrm{min}$, both bypass valve control and inventory control are used 


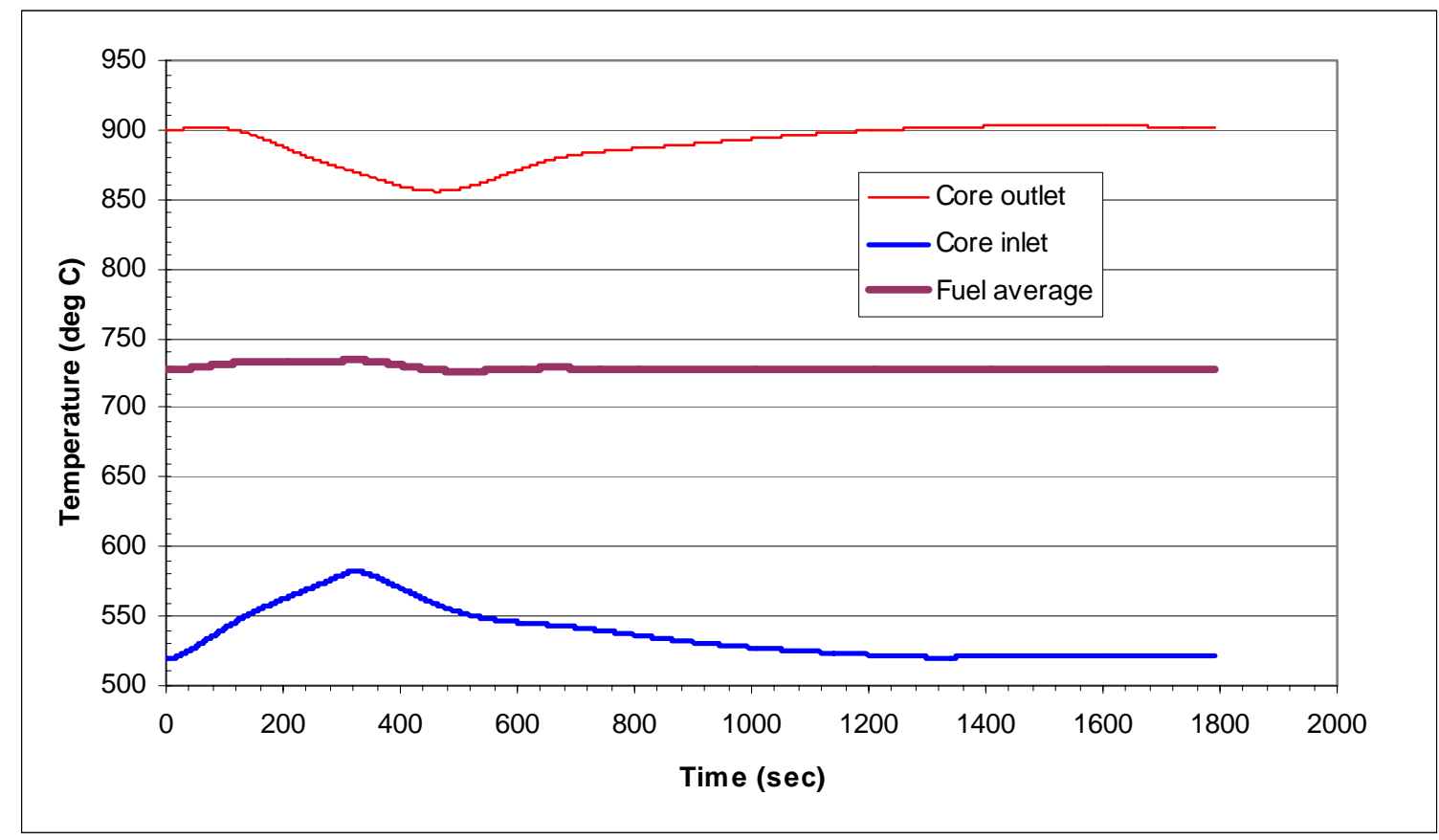

Figure 6.47 Core outlet/inlet temperature and fuel average temperature in a load ramp from $100 \%$ to $50 \%$ at a rate of $10 \% / \mathrm{min}$, both bypass control and inventory control are used

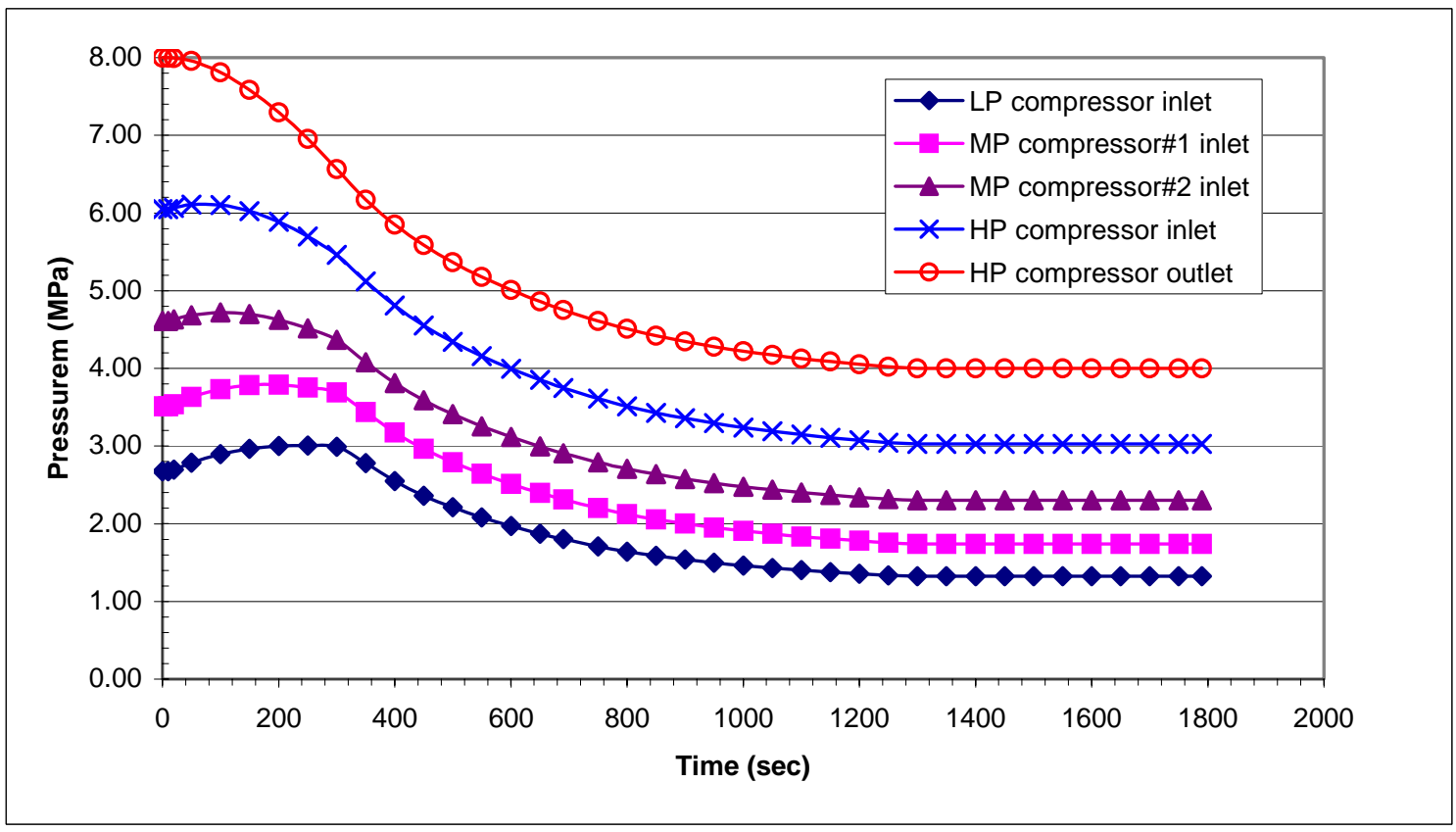

Figure 6.48 Pressures of compressors in a load ramp from $100 \%$ to $50 \%$ at a rate of $10 \% / \mathrm{min}$, both bypass valve control and inventory control are used 


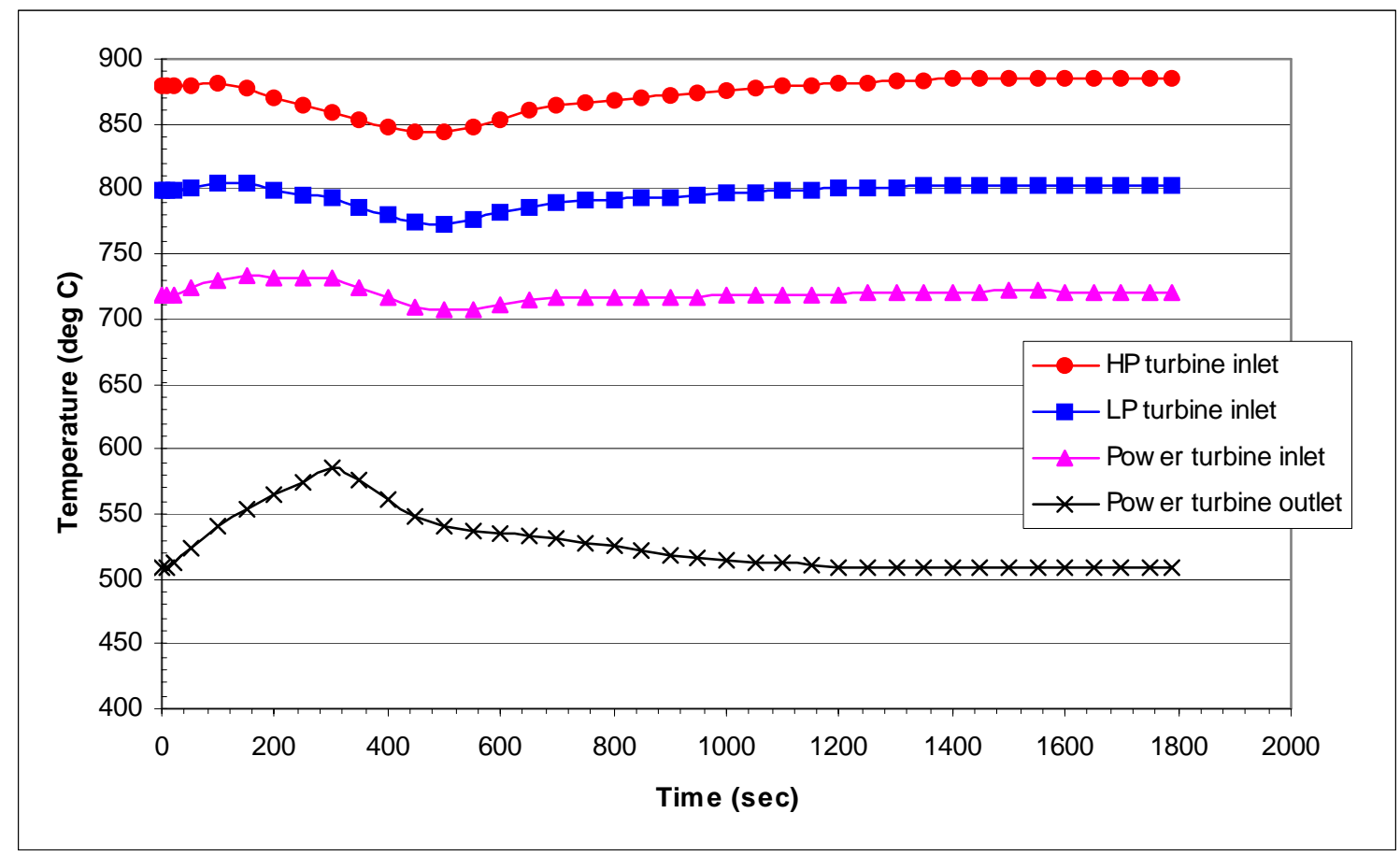

Figure 6.49 Turbine temperatures in a load ramp from $100 \%$ to $50 \%$ at a rate of $10 \% / \mathrm{min}$, both bypass valve control and inventory valve control are used

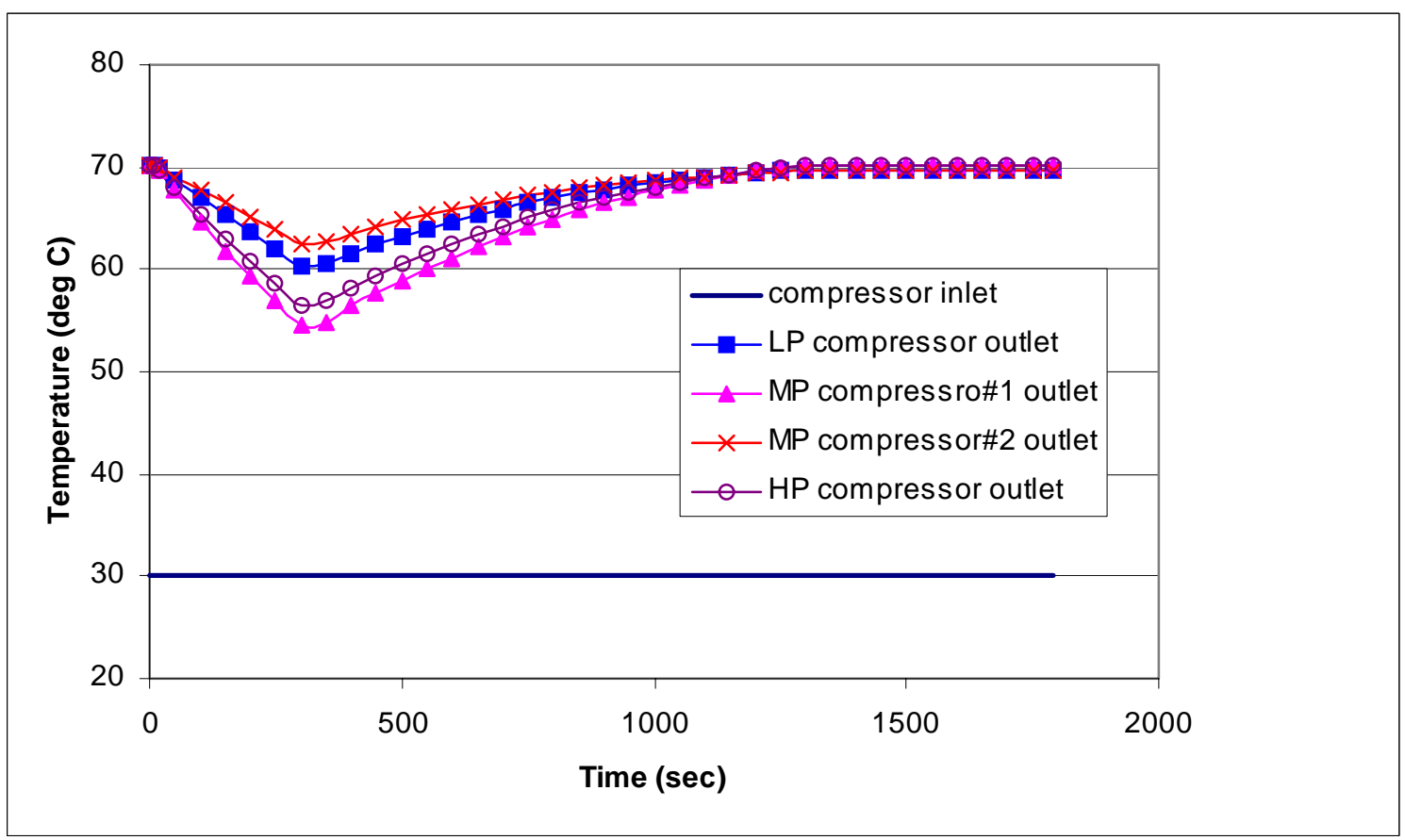

Figure 6.50 Compressor temperatures in a load ramp from $100 \%$ to $50 \%$ at a rate of $10 \% / \mathrm{min}$, both bypass valve control and inventory control are used 


\subsubsection{Grid Separation}

In the event of grid separation, the load out of the plant suddenly disappears. The only load on the generator is the power provided for the circulator and the station load, roughly about 10MWe. As the grid load suddenly vanishes, the excess power produced by the power turbine speeds up the turbine-generator shaft. Usually, when the shaft speed is over $120 \%$ of the nominal value, it can cause turbine blade damage due to the high centrifugal force. For over-speed protection, one method is quickly connecting the generator to an extra power consumption component, a water cooled resistor heater. The resistor provides a load ramp with $80 \%$ of full load at the grid separation, then gradually decreasing to $10 \%$ of full load in 10 seconds. During the 10 seconds, the resistor generates about 600 MJ heat, which can vaporize $232 \mathrm{~kg}$ water with initial temperature $20^{\circ} \mathrm{C}$, or can increase about $2400 \mathrm{~kg}$ water temperature from $20^{\circ} \mathrm{C}$ to $80^{\circ} \mathrm{C}$.

Figures 6.51 to 6.54 show the simulation results with centrifugal compressor map. The turbine-generator shaft speed peaks at $102.7 \%$ (3696rpm) at 21 seconds, much less than the limitation. The HP turbine shaft speed and LP turbine shaft speed drop to 3889rpm and $2826 \mathrm{rpm}$, respectively. The fission power decreases to about $18 \%$ of full power level after 320 seconds. The bypass valve mass flowrate is large, $38 \mathrm{~kg} / \mathrm{s}$.

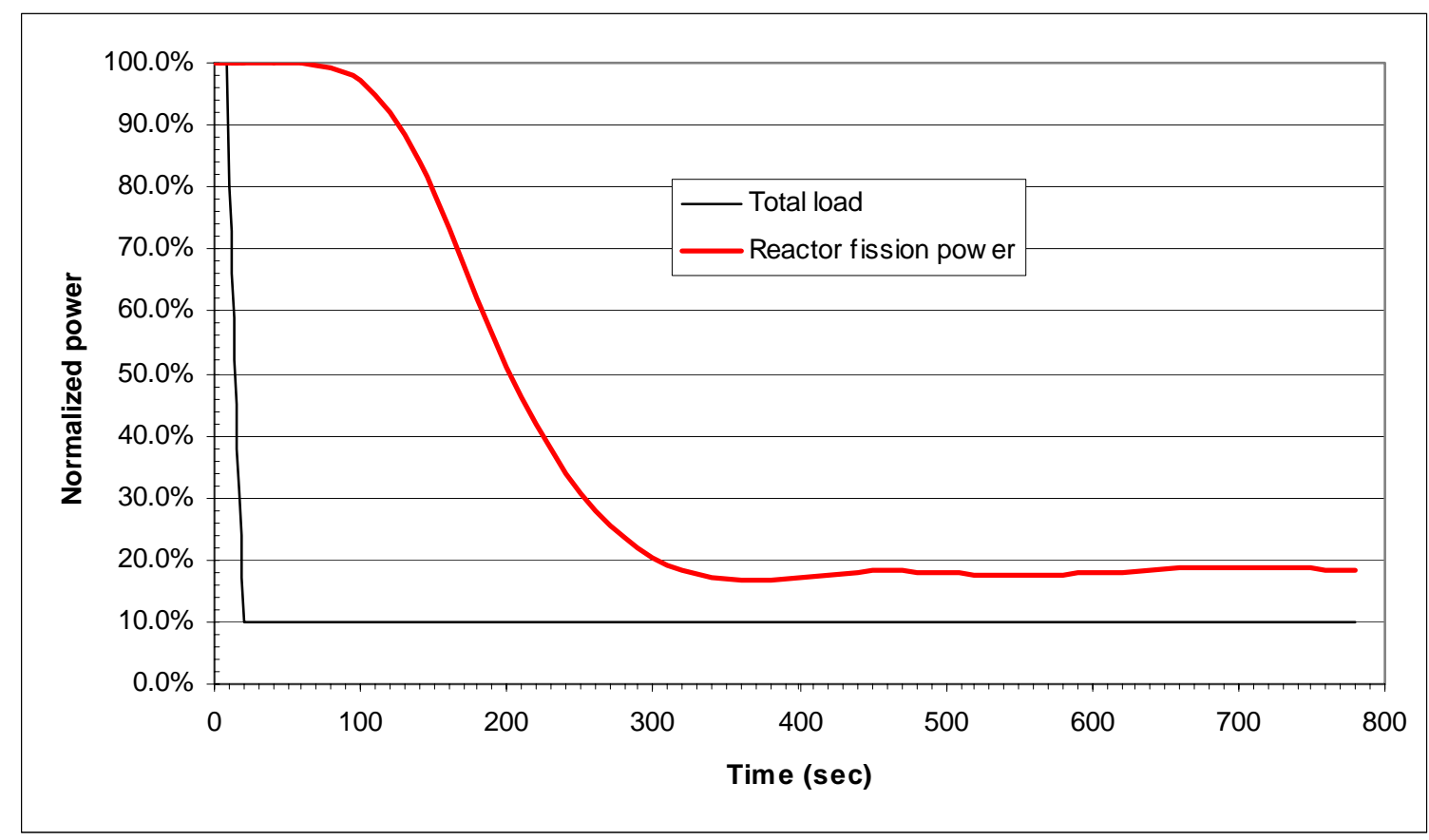

Figure 6.51 Total load and reactor fission power in a simulated grid separation, bypass valve control is used and centrifugal compressor maps are used 


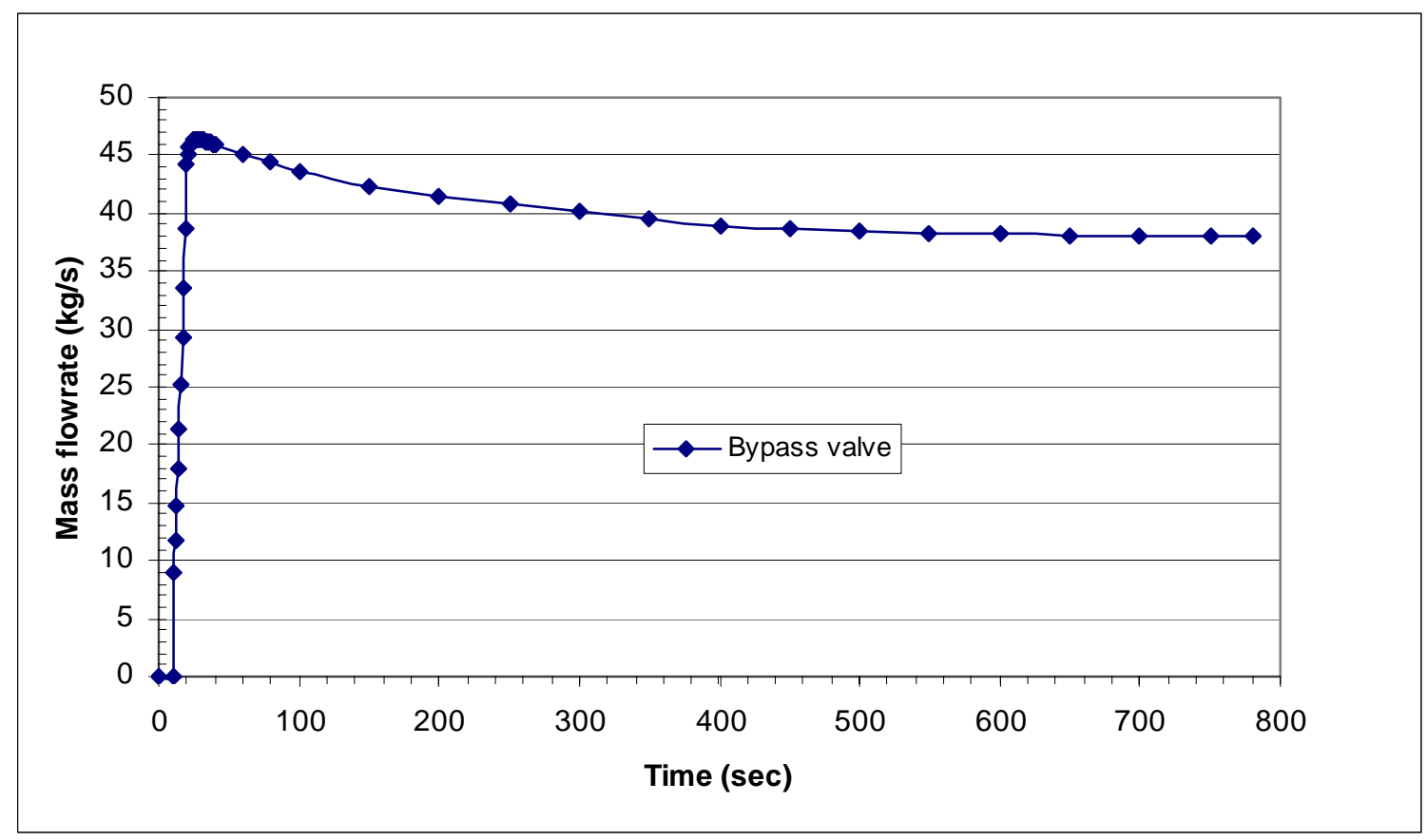

Figure 6.52 Bypass valve mass flowrate in a simulated grid separation, bypass valve control is used and centrifugal compressor maps are used

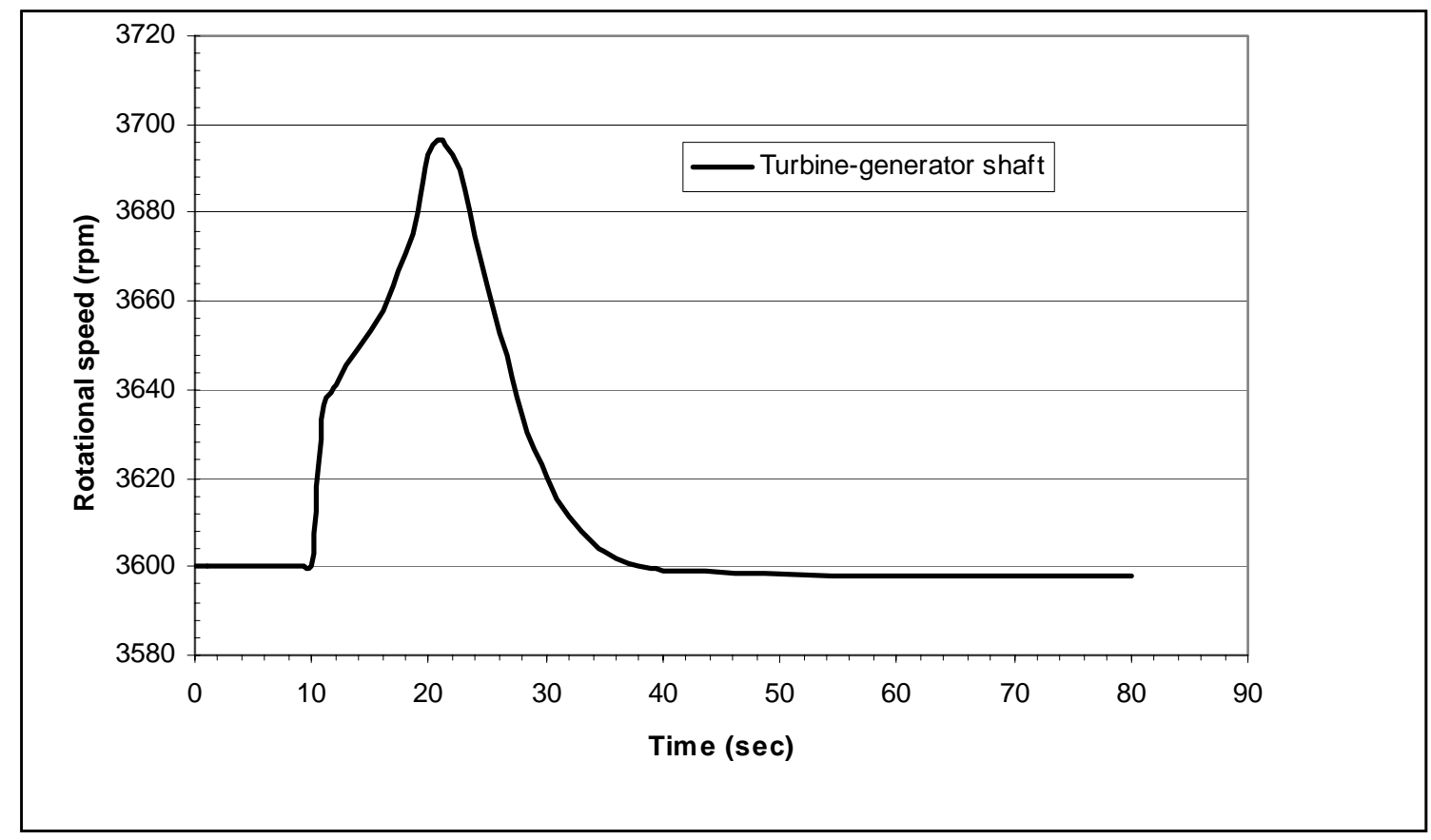

Figure 6.53 Turbine-generator shaft speed in a simulated grid separation, bypass valve control is used and centrifugal compressor maps are used - short time period 


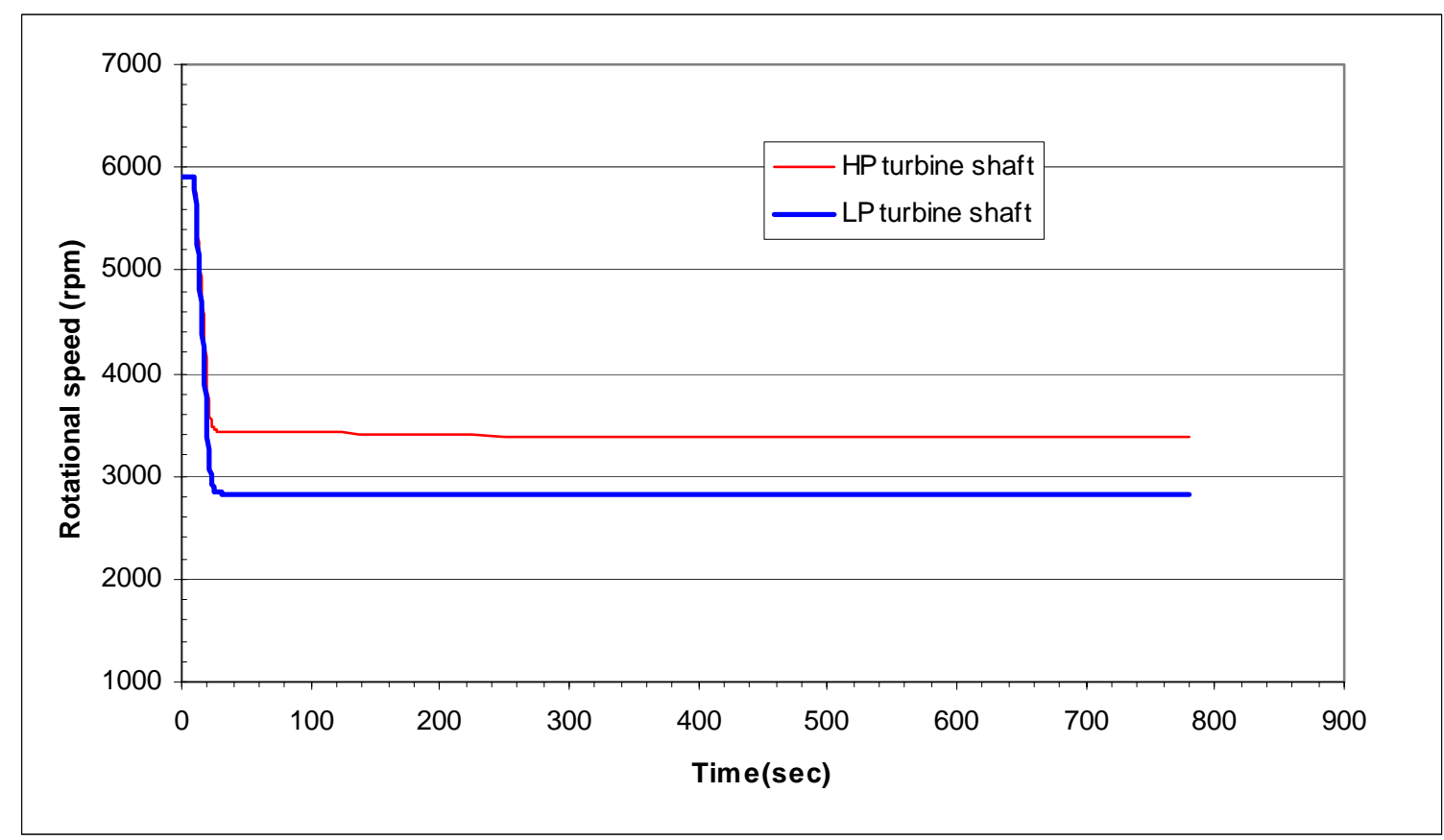

Figure 6.54 HP turbine shaft speed and LP turbine shaft speed in a simulated grid separation, bypass valve control is used and centrifugal compressor maps are used.

\subsection{Conclusion}

\subsubsection{Cycle Design Results}

For the power conversion system, we will use an indirect cycle, in which an IHX is used to separate the nuclear side and the PCU. For this configuration the components in the PCU are free of contamination and thus are easier to for maintain. A three-shaft arrangement is utilized and the shafts are in horizontal layout. Two sets of turbines will be used as the power turbine so that each has lower maximum power output. The selection makes the power output of the turbines close to the practical power output of manufacturers' current turbine technology and thus the R\&D cost is low. The three-shaft arrangement also makes the HP turbine shaft and the LP turbine shaft float, improving the operational stability. For the horizontal layout, the vibration problem is less serious than that of vertical layout and the selection of magnetic bearings is relatively easier than that for vertical layout. Considering the goal of high efficiency, all the turbines and compressors are axial type. The core outlet and inlet temperatures are chosen as $900^{\circ} \mathrm{C} / 520^{\circ} \mathrm{C}$, respectively. A separate cooling heat exchanger is used to provide a cooling stream for cooling the RPV and to ensure the RPV temperature is $280^{\circ} \mathrm{C}$ during normal operating. Three intercoolers are used to cool the compressor inlet helium. The intercoolers and precooler are helium/water heat exchangers, for which $27^{\circ} \mathrm{C}$ cooling water is provided by a cooling tower. A recuperator is used to recover the energy exhausted from the power turbine. The recuperator will be the plate-fin configuration (PFHX, Ingersoll Rand Energy System). There are two options for the IHX the printed circuit configuration (PCHX, Heatric) and the plate-fin configuration (PFHX, Ingersoll Rand Energy System). The printed circuit configuration is large and costly, but 
presents less development risk at the present time. The plate-fin configuration is promising in terms of the volume and cost.

As calculated in Section 4, the core pressure loss is about 1\%. For the IHX, using the printed circuit configuration with $95 \%$ effectiveness, the primary side pressure loss is $1.77 \%$ and $2 \%$ on the secondary side. If using the plate-fin configuration, the pressure losses are $0.5 \%$ and $1.4 \%$ on the primary side and secondary side, respectively. For the recuperator, the lowpressure side pressure loss is $0.8 \%$ and high-pressure side pressure loss is $0.33 \%$. The helium side pressure loss of the precooler is $0.8 \%$. For the intercooler between the LP compressor and the MP compressor \#1, the helium side pressure loss is $0.5 \%$. For the other two intercoolers, the helium side pressure losses are both $0.3 \%$ since they are in a relative high pressure position. The polytropic efficiency for all the turbines is $92 \%$, and $90 \%$ for all the compressors. The circulator isentropic efficiency is $90 \%$. The efficiency of the motor driving the circulator is $98 \%$. The generator efficiency is $98.5 \%$. The mechanical loss for the shaft is assumed to be $1 \%$.

When speaking of the cooled turbine, we mean using a substantial quantity of coolant to cool the nozzle and rotor blades themselves. In the MPBR, the highest working fluid temperature is lower than $880^{\circ} \mathrm{C}$, thus no cooling of nozzle and rotor blades is required. It is, however, the practice to pass a quantity of cooling working fluid over the turbine disc and blade roots. The amount of cool working fluid is about $1 \sim 2 \%$ of the total mass flowrate. In this work, we assume that $1.5 \%$ extra helium is bled from the HP compressor to cool the HP turbine disc and blade roots. For the LP turbine and power turbine, $1.5 \%$ extra helium from the MP compressor \#1 is individually passed to each turbine. Though using labyrinth seals for the turbomachines, there is still leakage. We assume 1\% extra helium leakage from the HP compressor and MP compressor \#1, respectively. For the IHX vessel, insulation is used to help cool the pressure boundary temperature to $150^{\circ} \mathrm{C}$. In normal conditions, no cooling helium is required. However, if there is a failure in the insulation material, cold helium is necessary to cool the IHX pressure boundary. For the casing of the turbines, a separate water cooling system might be the solution. The energy loss caused by the turbine casing cooling needs further study. For conservatism, we assume another $1.5 \%$ of extra helium is diverted from the HP compressor for cooling purposes. As a consequence, $4 \%$ extra helium mass flowrate is bled from the HP compressor and MP compressor\#2, respectively, because of cooling and leakage.

If using a printed circuit heat exchanger as the IHX, the gross electric power output is 131.4MW. Cycle pressure ratio is 2.92. Taking into account losses, the net electric power output is $121.3 \mathrm{MW}$. The plant net efficiency is $48.5 \%$. If using a plate-fin heat exchanger as the IHX, the gross electric power output is $130.61 \mathrm{MW}$. Cycle pressure ratio is 2.85 . The net electric power output is $123.5 \mathrm{MW}$. The plant net efficiency is $49.4 \%$. The salient parameters are summarized in Table 6.2. The cycle parameters are shown in Figure 6.55 and 6.56.

Currently, according to the information from ESKOM, the pebble bed reactor designer in South Africa, after considering all the losses, the PBMR net efficiency is around $41 \%$. Mainly two facts contribute to the higher efficiency of the MPBR: (1) Higher turbomachinery efficiency; in the MPBR, the turbine polytropic efficiency is $92 \%(92.5 \%$ 
isentropic efficiency) and the compressor polytropic efficiency is $90 \%(89.5 \%$ isentropic efficiency) while the turbine isentropic efficiency is $89 \%$ and the compressor isentropic efficiency is $89 \%$ in the PBMR[2]. (2) More intercooling stages; three intercooling stages is used in the MPBR while one is used in the PBMR

For the MPBR, after considering the losses caused by the turbine casing cooling, the plant net efficiency is believed to be around $45 \%$.

\subsubsection{Control System Results}

The turbines are designed with 50\% reaction. Turbine and compressor sets are on individual shafts. For the shaft, the axial force caused by the turbine is balanced by that of compressors, thus the axial direction net force on the shaft is zero.

The plant transient analyses show that, for normal operating transients, the pebble bed reactor coupled with an indirect, three-shaft arrangement power conversion system is stable and controllable. It meets the load following requirement. Bypass valve control is used for fast response while inventory control is used for slow, partial load operation. For the threeshaft arrangement, the bypass valve control does not decrease the cycle efficiency greatly. However, solely using bypass valve control for partial load operation increases the core inlet temperature. This happens using either a direct, single-shaft arrangement cycle or an indirect, multi-shaft arrangement cycle. Inventory control allows the partial load operation with high efficiency and without thermal stress on the components.

\subsection{Summary}

This section provides steady state parametric analyses for cycle design. The plant nominal parameters and performances are given for an indirect, intercooled and recuperated cycle design. Even considering 4\% extra helium mass flowrate for leakage and cooling purposes from the HP compressor and MP compressor\#1, the plant net efficiency can reach $48 \%$. The dynamic model has been verified with Flownet. Four load transient scenarios have been simulated. For plant normal operating load transients, the plant incorporates the pebble bed reactor and the indirect, three-shaft arrangement power conversion system is stable and controllable. 
Table 6.2 MPBR design parameters

\begin{tabular}{|c|c|c|}
\hline Thermal power & \multicolumn{2}{|c|}{250 MWth } \\
\hline Core outlet/inlet temperature & \multicolumn{2}{|c|}{$900 / 520^{\circ} \mathrm{C}$} \\
\hline Pressure ratio of PCU & 2.92 & 2.86 \\
\hline $\begin{array}{l}\text { Helium mass flowrate } \\
\text { (primary/secondary) }\end{array}$ & \multicolumn{2}{|c|}{$126.7 / 126.7 \mathrm{~kg} / \mathrm{s}$} \\
\hline System maximum pressure & \multicolumn{2}{|c|}{$8.0 \mathrm{MPa}$} \\
\hline Core pressure drop & \multicolumn{2}{|c|}{$1 \%$} \\
\hline IHX & $\begin{array}{l}\text { Printed circuit HX } \\
\text { Effectiveness: } 95 \% \\
\text { Pressure drop: } \\
1.77 \% \text { (hot side) } \\
2 \% \text { (cold side) }\end{array}$ & $\begin{array}{l}\text { Plate-fin HX } \\
\text { Effectiveness: } 95 \% \\
\text { Pressure drop: } \\
0.5 \% \text { (hot side) } \\
\quad 1.4 \% \text { (Cold side) }\end{array}$ \\
\hline Recuperator (Plate-fin HX) & \multicolumn{2}{|c|}{$\begin{array}{l}\text { Effectiveness: } 95 \% \\
\text { Pressure drop: } \\
\qquad \begin{array}{l}0.8 \% \text { (low-pressure side) } \\
0.33 \% \text { (high-pressure side) }\end{array}\end{array}$} \\
\hline Precooler & \multicolumn{2}{|c|}{ Helium side pressure drop: $0.8 \%$} \\
\hline Intercooler \#1 & \multicolumn{2}{|c|}{ Helium side pressure drop: $0.5 \%$} \\
\hline Intercooler \#2, \#3 & \multicolumn{2}{|c|}{ Helium side pressure drop: $0.3 \%$} \\
\hline Turbine polytropic efficiency & \multicolumn{2}{|c|}{$92 \%$} \\
\hline Compressor polytropic efficiency & \multicolumn{2}{|c|}{$90 \%$} \\
\hline Generator efficiency & \multicolumn{2}{|c|}{$98.5 \%$} \\
\hline Circulator isentropic efficiency & \multicolumn{2}{|c|}{$90 \%$} \\
\hline Circulator motor efficiency & \multicolumn{2}{|c|}{$98 \%$} \\
\hline Turbine shaft mechanical loss & \multicolumn{2}{|c|}{$1 \%$} \\
\hline $\begin{array}{l}\text { HP compressor and MP compressor \#1 } \\
\text { extra leakage rate }\end{array}$ & \multicolumn{2}{|c|}{$1 \%$} \\
\hline $\begin{array}{l}\text { HP compressor and MP compressor \#1 } \\
\text { extra cooling rate }\end{array}$ & \multicolumn{2}{|c|}{$3 \%$} \\
\hline \multicolumn{3}{|l|}{ Losses } \\
\hline Circulator power & $6.58 \mathrm{MWe}$ & $3.57 \mathrm{MWe}$ \\
\hline Other station load & \multicolumn{2}{|c|}{$2.5 \mathrm{MWe}$} \\
\hline Switch-yard loss & \multicolumn{2}{|c|}{$0.6 \%$} \\
\hline System radiation loss & \multicolumn{2}{|c|}{0.5 MWth } \\
\hline Gross power output & $131.4 \mathrm{MWe}$ & $130.6 \mathrm{MWe}$ \\
\hline $\begin{array}{l}\text { Net electric power (without considering } \\
\text { the energy loss for turbine casing } \\
\text { cooling) }\end{array}$ & $121.3 \mathrm{MWe}$ & $123.5 \mathrm{MWe}$ \\
\hline $\begin{array}{l}\text { Net plant efficiency (without } \\
\text { considering the energy loss for turbine } \\
\text { casing cooling) }\end{array}$ & $48.5 \%$ & $49.4 \%$ \\
\hline
\end{tabular}




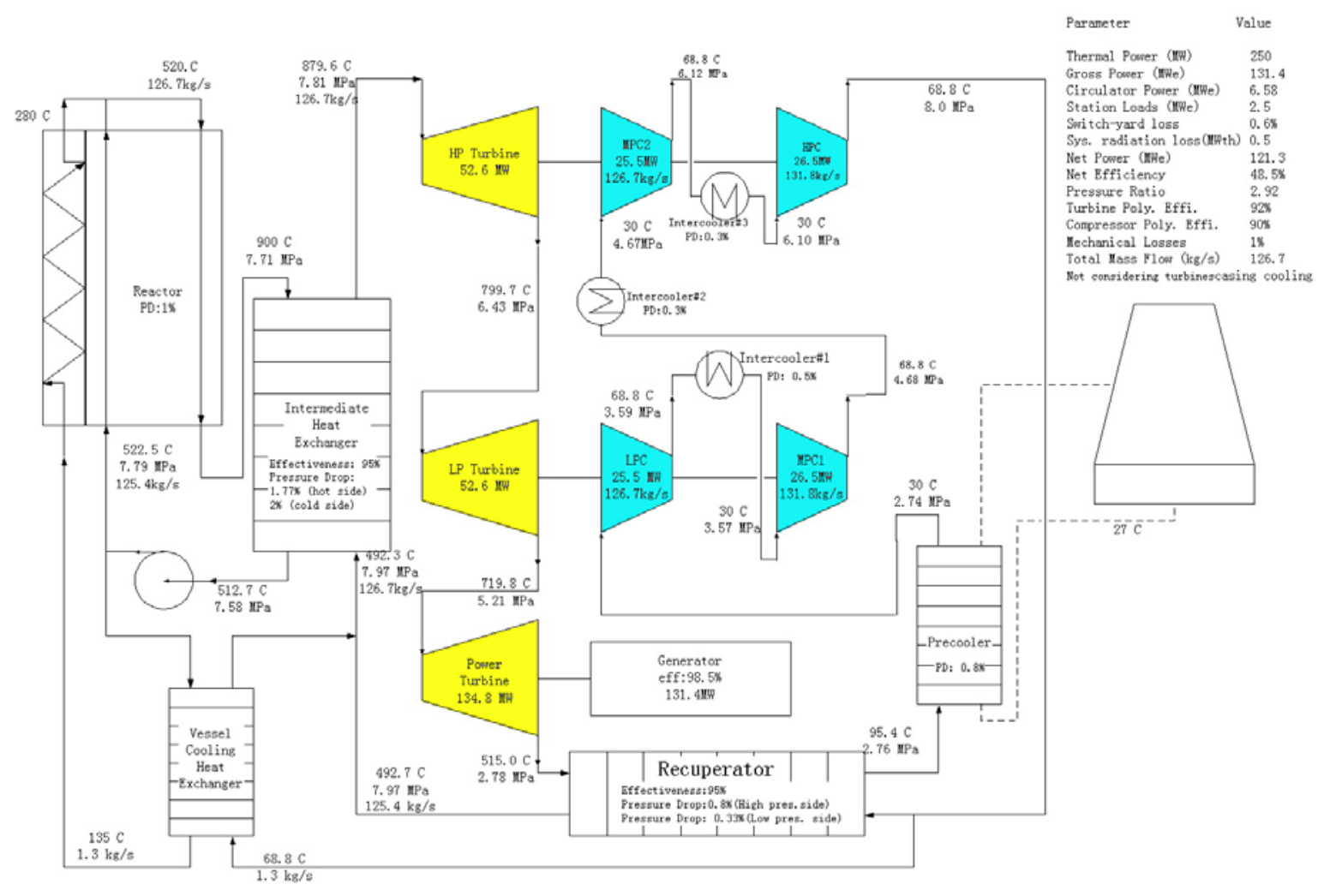

Figure 6.55 Cycle parameters using printed circuit heat exchanger as IHX

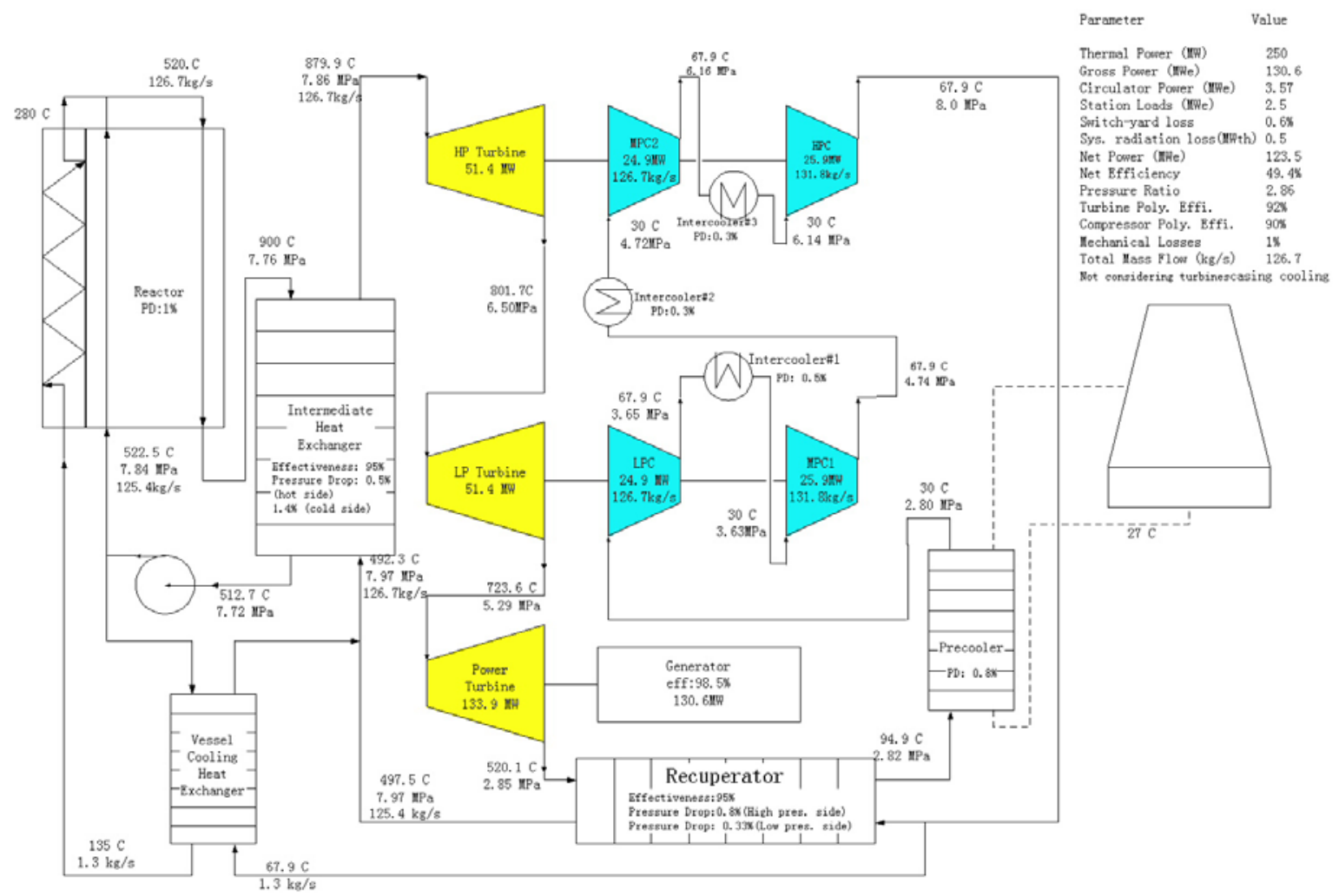

Figure 6.56 Cycle parameters using plate-fin heat exchanger as IHX 


\section{References: Section 6}

[1] P. P. Walsh, P. Fletcher, “Gas turbine performance”, Blackwell Science, 1998.

[2] IAEA, "Current status and future development of modular high temperature gas cooled reactor technology”, IAEA-TECDOC-1198,

http://www.iaea.or.at/inis/aws/htgr/abstracts/abst_gcr review.html. 


\section{Summary and Conclusions}

\subsection{Summary of Conclusions}

For the power conversion system of the MPBR, an indirect recuperated and intercooled Brayton cycle is utilized. With regard to the turbomachinery layout, a horizontal three-shaft arrangement has been developed. There are two options for the IHX configuration - the printed circuit heat exchanger of Heatric and the plate-fin heat exchanger of Ingersoll Rand Energy System. The printed circuit heat exchanger is large, heavy and costly, but with extensive operating experience in high temperature and high pressure environments. The plate-fin heat exchanger is relatively small, light and cheap, and with low pressure drop. For the plate-fin configuration, a thermal parametric analysis has been conducted. The turbines and compressors are all axial type due to their higher efficiency. For the axial compressor, its working range is narrow compared with the centrifugal type. However, the three-shaft arrangement provides improved operational stability. A recuperator and intercoolers are used to improve the cycle efficiency. For the recuperator, a plate-fin heat exchanger is used. Its thermal design has also been performed. From the heat exchanger thermal analysis, we can see that the heat exchanger volume, and the cost are roughly doubled when the effectiveness increases from $90 \%$ to $95 \%$. Based on the cycle parametric analysis using the steady state model, the core outlet/inlet temperatures are chosen as $900^{\circ} \mathrm{C}$ and $520^{\circ} \mathrm{C}$, respectively. For the indirect cycle design, the pressure losses in the primary system can significantly affect the consumed power of the circulator, and thus the plant net efficiency. In terms of the plant efficiency, using a plate-fin heat exchanger as the IHX offers higher plant net efficiency.

For meeting ASME code section III, RPV cooling is deployed. A separate small IHX is used to provide cooling helium in the primary system. In practice, on the PCU side helium is bled from the compressor outlet for cooling purposes, i.e., cooling the turbine disc and blade roots. From the parametric analysis, the bleeding and the leakage do not greatly affect the cycle efficiency.

A cooling tower is used to provide $27^{\circ} \mathrm{C}$ water for cooling the compressor inlet helium in the precooler and intercoolers. The compressor inlet helium is cooled to $30^{\circ} \mathrm{C}$. This design can be implemented in districts where rivers, lakes and the sea are not available. Based on the feasibility study conducted in collaboration with Concepts-NREC, MA, for the key components such as turbomahines, IHX and recuperator, and the assumption of $4 \%$ extra helium massflowrate bled from the HP compressor and MP compressor\#1, the plant net efficiency is over $48 \%$. Thus, it fulfills the design objective -- $45 \%$ plant net efficiency.

For investigating the controllability and operational stability of the plant, a dynamic model, MPBRSim, has been developed. The dynamic model incorporates the reactor core and PCU to predict their interaction. The model includes the components of the plant: reactor, heat exchangers, turbomachines, valves, pipes and controllers. Verification has been conducted against Flownet simulation results. Both codes exhibit the same trend for the response to a load transient. 
Two control methods, bypass valve control and inventory control, are available for the gas turbine cycle. For normal operating load transients, solely using the bypass valve can maintain the power turbine shaft speed within its limitations, and will not degrade the cycle efficiency greatly since the two other shafts are floating. However, it increases the recuperator low-pressure side inlet temperature and the core inlet temperature. For long time partial load operation, the increased temperatures are not allowed. Inventory control is used to reduce the cycle mass flowrate and to make the bypass valve "feathered". During partial load operation, using inventory control, the temperatures around the cycle are close to the nominal values and the cycle efficiency remains high.

The control strategy and the control objective are defined for the MPBR. By using the designed control scheme, the simulation results show that the control system meets the load following requirement and the automatic operation within the control band. The control system enables the plant to operate with $10 \%$ step load change and $10 \% / \mathrm{min}$ load decrease ramp as well as 5\%/min load increase ramp in the power range of $100 \% \sim 50 \%$. In the grid separation event, a turbomachine braking method is proposed to prevent the turbinegenerator speed from exceeding $120 \%$.

An array of load transients has been simulated using MPBRSim, both for an axial compressor map and a centrifugal compressor map. The simulation results demonstrate the controllability and operational stability for the indirect, three-shaft arrangement cycle design.

\subsection{Discussion And Recommendation}

The plant efficiency prediction has not taken into account the loss caused byturbine casing cooling. This cooling can be implemented by a separate water cooling system or a separate helium cooling system. The cooling helium also can be helium diverted from the HP compressor outlet. The cooling scheme is to be determined in further investigations. A $1 \%$ leakage rate is assumed for both the HP compressor and MP compressor \#1. More accurate estimation of leakage rate will require an investigation of sealing technology. For the indirect cycle design, the IHX is the key component. Incoloy $800 \mathrm{HT}$ is expected to be the IHX material. It requires that the maximum differential pressure between the primary and the secondary sides will not exceed 1.0 MPa at the temperature condition of the MPBR. This requirement can be met during normal operation. However, in extreme transients such as when the secondary side has been depressurized while the primary side remains hot and at pressure, the stress-time envelope must be limited to prevent excessive deformation. Removal of this restriction will require the qualification of more advanced material under ASME code section III.

In the dynamic model, the axial compressor map and centrifugal map are both used. Because of the high steepness of the axial compressor speed lines in the map, the calculation time step size needs to be very small to obtain the convergent results. This is a drawback for realtime simulation. A procedure should be added to calculate the choking flow condition which can occur in the compressors and turbines during total load rejection instantaneously. For 
future research, improving the solution algorithm is one way to implement real-time simulation. In the core model, the calculation of temperature reactivity feedback is based on the average core temperature. This mechanism is considered sufficient for reactor power control. For more detailed core analysis, the uneven reactivity distribution caused by temperature profile change can be taken into considered.

Currently, PI controllers are used. It can basically satisfy the plant control requirements. For obtaining superior control performance, a more sophisticated control system design can be performed based on the calculated results of transients using the dynamic model. For the gas turbine nuclear power plant, startup and shutdown procedures are of interest for cycle design. Optimizing the volume and the number of inventory vessels is required. These tasks are left for future research. 


\section{Appendix A Concepts-NREC Heat Exchanger Design}

Initial Intermediate Heat Exchanger (IHX) thermal design has been conducted by ConceptsNREC for two configurations - Printed Circuit Heat Exchanger (PCHE) and Plate Fin Heat Exchanger (PFHE). Additional IHX and recuperator designs with the PCHE configuration for parametric variations have been performed. The IHX designs assumed using Incoloy 800 as the material of construction while the recuperators were constructed of 347 stainless steel.

The PCHE design calculations were based on the following standard plate and flow-passage detailed dimensions:

- Channel diameter $=2 \mathrm{~mm}$

- Plate thickness $=1.6 \mathrm{~mm}$

- Center-to-center channel spacing $=2.44 \mathrm{~mm}$

The PFHX design calculations used the detailed plate and fin geometry:

- Cold-side fin layers $=1$

- Hot-side fin layers $=2$

- Plate spacing $=0.065$ in

- Parting plate thickness $=0.015$ in

- Fin material thickness $=0.003$ in

- Fin spacing $=45$ fins/inch

-

The initial design conditions for the IHX are listed in Table A.1. Tables A.2 and A.3 list the IHX initial parametric results for the PCHX and PFHX, respectively. Table A.4 lists the initial IHX nominal results. The designs and package approaches for the two types are illustrated in Figures A.1 and A.2 for the PCHE and Figures A.3 and A.4 for the PFHX.

The additional parametric design calculation conditions and results are listed in Tables A.5 to A.7. Note that in Table A.7, the plate-fin type results are obtained by scaling the initial IHX results.

Table A.1 - Initial IHX Design Conditions

\begin{tabular}{|l|c|c|}
\hline & $\begin{array}{c}\text { Primary } \\
\text { (Hot Side) } \\
\text { Loop }\end{array}$ & $\begin{array}{c}\text { Secondary } \\
\text { (Cold Side) } \\
\text { Loop }\end{array}$ \\
\hline Fluid & Helium & Helium \\
\hline $\begin{array}{l}\text { Flow Rate, kg. sec } \\
(\mathrm{lbm} / \mathrm{sec})\end{array}$ & $119(262)$ & $119(262)$ \\
\hline Inlet Temperature, C (F) & $850(1562)$ & $386(726)$ \\
\hline Outlet Temperature, C (F) & $432(810)$ & $803(1478)$ \\
\hline Inlet Pressure, MPa (psia) & $7.56(1097)$ & $7.85(1139)$ \\
\hline
\end{tabular}

Effectiveness

Maximum Primary Pressure Loss

Maximum Secondary Pressure Loss
$90 \%$ $2.0 \%$ 
Table A.2 - Initial Parametric Results for PCHE IHX

\begin{tabular}{|c|c|c|c|c|c|c|c|c|c|}
\hline Flow Rate, $\mathrm{m}_{\mathrm{c}}$, Ibm/sec & 262 & 262 & 262 & 262 & 262 & 262 & 262 & 262 & 262 \\
\hline Inlet Pressure, $P_{c, i n}$, psia & 1139 & 1139 & 1139 & 1139 & 1139 & 1139 & 1139 & 1139 & 1139 \\
\hline Inlet Temperature, $T_{c, i n}$, deg F & 726.5 & 726.5 & 726.5 & 726.5 & 726.5 & 726.5 & 726.5 & 726.5 & 726.5 \\
\hline Outlet Temperature, $\mathrm{T}_{\mathrm{c}, \mathrm{out}}$, deg F & 1478.0 & 1478.0 & 1478.0 & 1478.0 & 1478.0 & 1478.0 & 1478.0 & 1478.0 & 1478.0 \\
\hline Flow Rate, $\mathrm{m}_{\mathrm{h}}$, lbm/sec & 262 & 262 & 262 & 262 & 262 & 262 & 262 & 262 & 262 \\
\hline Inlet Pressure, $\mathrm{P}_{\mathrm{h}, \mathrm{in}}$, psia & 1097 & 1097 & 1097 & 1097 & 1097 & 1097 & 1097 & 1097 & 1097 \\
\hline Inlet Temperature, $T_{h, i n}$, deg $F$ & 1562.0 & 1562.0 & 1562.0 & 1562.0 & 1562.0 & 1562.0 & 1562.0 & 1562.0 & 1562.0 \\
\hline Outlet Temperature, $\mathrm{T}_{\mathrm{h}, \text { out }}$, deg F & 810.0 & 810.0 & 810.0 & 810.0 & 810.0 & 810.0 & 810.0 & 810.0 & 810.0 \\
\hline Core Height, in & 566.40 & 566.40 & 566.40 & 566.40 & 708.00 & 708.00 & 708.00 & 708.00 & 708.48 \\
\hline Core Width, in & 23.60 & 23.60 & 23.60 & 23.60 & 23.60 & 23.60 & 23.60 & 23.60 & 23.60 \\
\hline Core Length, in & 17.46 & 17.46 & 17.46 & 17.46 & 15.83 & 15.83 & 15.83 & 15.83 & 15.83 \\
\hline Inlet Header w, in & 10.00 & 15.00 & 20.00 & 25.00 & 10.00 & 15.00 & 20.00 & 25.00 & 10.00 \\
\hline Outlet Header w, in & 10.00 & 15.00 & 20.00 & 25.00 & 10.00 & 15.00 & 20.00 & 25.00 & 10.00 \\
\hline HT Area $A_{c}, \mathrm{ft}^{\wedge} 2$ & 18292 & 18292 & 18292 & 18292 & 20731 & 20731 & 20731 & 20731 & 20745 \\
\hline HT Area $A_{h}, \mathrm{ft}^{\wedge} 2$ & 18292 & 18292 & 18292 & 18292 & 20731 & 20731 & 20731 & 20731 & 20745 \\
\hline Plate Spacing, $b_{c}$, in & 0.03900 & 0.03900 & 0.03900 & 0.03900 & 0.03900 & 0.03900 & 0.03900 & 0.03900 & 0.03900 \\
\hline Channel Dia, $\mathrm{d}_{\mathrm{c}}$, in & 0.07900 & 0.07900 & 0.07900 & 0.07900 & 0.07900 & 0.07900 & 0.07900 & 0.07900 & 0.07900 \\
\hline Channel Spacing, $S_{p, c}$, in & 0.09600 & 0.09600 & 0.09600 & 0.09600 & 0.09600 & 0.09600 & 0.09600 & 0.09600 & 0.09600 \\
\hline Free Flow/Face Area, $\sigma_{c}$ & 0.402 & 0.402 & 0.402 & 0.402 & 0.402 & 0.402 & 0.402 & 0.402 & 0.402 \\
\hline Surface/Volume, $\beta_{\mathrm{c}}, \mathrm{ft}^{\wedge} 2 / \mathrm{ft}^{\wedge} 3$ & 433.4 & 433.4 & 433.4 & 433.4 & 433.4 & 433.4 & 433.4 & 433.4 & 433.4 \\
\hline Hydraulic Radius, $\mathrm{r}_{\mathrm{H}, \mathrm{c}}, \mathrm{ft}$ & $9.28 \mathrm{E}-04$ & $9.28 \mathrm{E}-04$ & $9.28 \mathrm{E}-04$ & $9.28 \mathrm{E}-04$ & $9.28 \mathrm{E}-04$ & $9.28 \mathrm{E}-04$ & $9.28 \mathrm{E}-04$ & $9.28 \mathrm{E}-04$ & $9.28 \mathrm{E}-04$ \\
\hline Plate Spacing, $b_{h}$, in & 0.03900 & 0.03900 & 0.03900 & 0.03900 & 0.03900 & 0.03900 & 0.03900 & 0.03900 & 0.03900 \\
\hline Channel Dia, $d_{h}$, in & 0.07900 & 0.07900 & 0.07900 & 0.07900 & 0.07900 & 0.07900 & 0.07900 & 0.07900 & 0.07900 \\
\hline Channel Spacing, $S_{p, h}$, in & 0.09600 & 0.09600 & 0.09600 & 0.09600 & 0.09600 & 0.09600 & 0.09600 & 0.09600 & 0.09600 \\
\hline Free Flow/Face Area, $\sigma_{\mathrm{h}}$ & 0.402 & 0.402 & 0.402 & 0.402 & 0.402 & 0.402 & 0.402 & 0.402 & 0.402 \\
\hline Surface/Volume, $\beta_{\mathrm{h}}, \mathrm{ft}^{\wedge} 2 / \mathrm{ft}^{\wedge} 3$ & 433.4 & 433.4 & 433.4 & 433.4 & 433.4 & 433.4 & 433.4 & 433.4 & 433.4 \\
\hline Hydraulic Radius, $\mathrm{r}_{\mathrm{H}, \mathrm{h}}$, ft & $9.28 \mathrm{E}-04$ & $9.28 \mathrm{E}-04$ & $9.28 \mathrm{E}-04$ & $9.28 \mathrm{E}-04$ & $9.28 \mathrm{E}-04$ & $9.28 \mathrm{E}-04$ & $9.28 \mathrm{E}-04$ & $9.28 \mathrm{E}-04$ & $9.28 \mathrm{E}-04$ \\
\hline UA, Btu/hr F & 10540800 & 10540800 & 10540800 & 10540800 & 10539000 & 10539000 & 10539000 & 10539000 & 10544400 \\
\hline $\mathrm{Q}, \mathrm{kBtu} / \mathrm{hr}$ & 880560 & 880560 & 880560 & 880560 & 880500 & 880500 & 880500 & 880500 & 880560 \\
\hline Effectiveness $\varepsilon, \%$ & 90.002 & 90.002 & 90.002 & 90.002 & 90.000 & 90.000 & 90.000 & 90.000 & 90.003 \\
\hline Reynolds Number, $\mathrm{Re}_{\mathrm{c}}$ & 2975.0 & 2975.0 & 2975.0 & 2975.0 & 2380.0 & 2380.0 & 2380.0 & 2380.0 & 2378.0 \\
\hline HT Coefficient, $h_{c}$, Btu/hr ft^2 F & 1347.0 & 1347.0 & 1347.0 & 1347.0 & 1164.0 & 1164.0 & 1164.0 & 1164.0 & 1164.0 \\
\hline Conductance, $\left(\eta_{\mathrm{o}} \mathrm{hA}\right)_{\mathrm{c}}, \mathrm{Btu} / \mathrm{hr} \mathrm{F}$ & 24648000 & 24648000 & 24648000 & 24648000 & 24132000 & 24132000 & 24132000 & 24132000 & 24138000 \\
\hline Cold $\Delta \mathrm{P}_{\mathrm{c}}, \%$ & 3.072 & 2.234 & 1.918 & 1.765 & 2.004 & 1.443 & 1.232 & 1.133 & 2.002 \\
\hline Inlet $\mathrm{Hdr} \Delta \mathrm{P}, \%$ & 0.588 & 0.284 & 0.169 & 0.113 & 0.394 & 0.190 & 0.113 & 0.076 & 0.393 \\
\hline Outlet Hdr $\Delta \mathrm{P}, \%$ & 1.031 & 0.497 & 0.296 & 0.198 & 0.690 & 0.333 & 0.198 & 0.137 & 0.689 \\
\hline Reynolds Number, $\mathrm{Re}_{\mathrm{h}}$ & 2867.0 & 2867.0 & 2867.0 & 2867.0 & 2293.0 & 2293.0 & 2293.0 & 2293.0 & 2292.0 \\
\hline HT Coefficient, $h_{h}$, Btu/hr ft^2 F & 1364.0 & 1364.0 & 1364.0 & 1364.0 & 1179.0 & 1179.0 & 1179.0 & 1179.0 & 1179.0 \\
\hline Conductance, $\left(\eta_{\mathrm{o}} \mathrm{hA}\right)_{\mathrm{h}}, \mathrm{Btu} / \mathrm{hr} \mathrm{F}$ & 24960000 & 24960000 & 24960000 & 24960000 & 24447000 & 24447000 & 24447000 & 24447000 & 24451200 \\
\hline Hot $\Delta \mathrm{P}_{\mathrm{h}}, \%$ & 2.638 & 3.118 & 3.598 & 4.078 & 1.733 & 2.068 & 2.403 & 2.739 & 1.731 \\
\hline No of Passage Pairs & 4496 & 4496 & 4496 & 4496 & 5620 & 5620 & 5620 & 5620 & 5624 \\
\hline HX Weight, Ibm & 79512 & 93984 & 108456 & 122952 & 93480 & 111570 & 129690 & 147780 & 93564 \\
\hline
\end{tabular}

Note: "c" = Cold Side, "h" = Hot Side 
Table A.3 - Initial Parametric Results for PFHE IHX

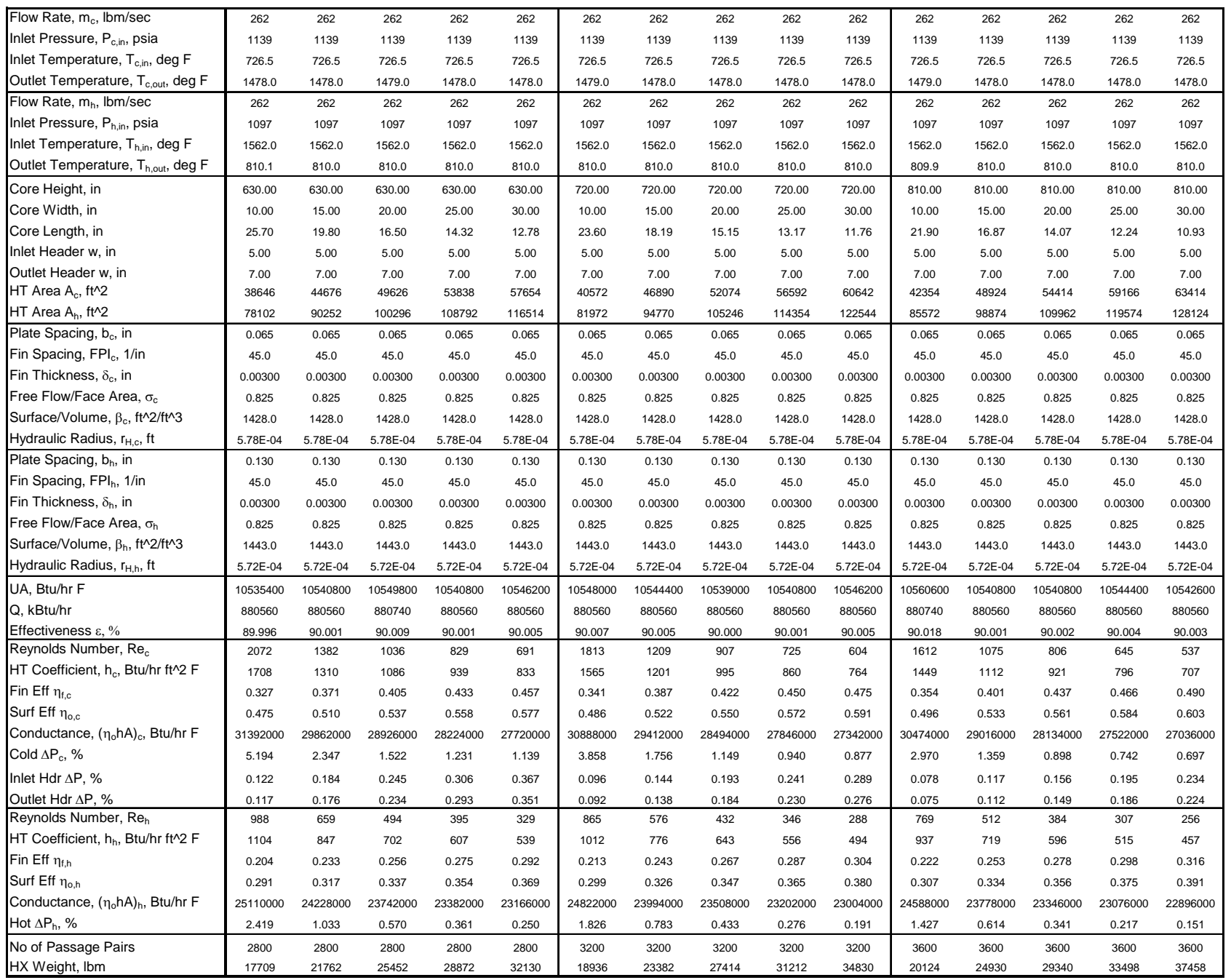

Note: "c" = Cold Side, "h" = Hot Side 
Table A.4 - Comparison of Initial Nominal IHX Designs

\begin{tabular}{|c|c|c|c|c|}
\hline & \multicolumn{3}{|c|}{ PCHE Design } & \multirow{2}{*}{$\begin{array}{c}\text { PFHE Design } \\
\text { Model Estimates }^{(2)}\end{array}$} \\
\hline & \multicolumn{2}{|c|}{ Model Estimates $^{(2)}$} & Heatric Quote ${ }^{(3)}$ & \\
\hline Number of Modules ${ }^{(1)}$ & \multicolumn{2}{|c|}{6} & 6 & $6 \times 3=18$ \\
\hline Number of Vessels & \multicolumn{2}{|c|}{6} & 6 & 6 \\
\hline \multirow[t]{2}{*}{ Nominal Core Dimensions $\mathrm{W} \times \mathrm{L} \times \mathrm{H}$} & \multicolumn{2}{|c|}{$23.6 \times 26.0 \times 39.4$ in } & $23.5 \times 30.2 \times 87.4$ in & $15 \times 17 \times 45$ in \\
\hline & \multicolumn{2}{|c|}{$600 \times 660 \times 3000 \mathrm{~mm}$} & $596 \times 766 \times 2220 \mathrm{~mm}$ & $381 \times 432 \times 1143 \mathrm{~mm}$ \\
\hline \multirow[t]{2}{*}{ Module Core Volume } & \multicolumn{2}{|c|}{$1.19 \mathrm{cu} \mathrm{m}$} & $1.01 \mathrm{cu} \mathrm{m}$ & $0.188 \mathrm{cu} \mathrm{m}$ \\
\hline & \multicolumn{2}{|c|}{$42.0 \mathrm{cu} \mathrm{ft}$} & $35.9 \mathrm{cu} \mathrm{ft}$ & $6.64 \mathrm{cu} \mathrm{ft}$ \\
\hline \multirow[t]{2}{*}{ Total IHX Core Volume } & \multicolumn{2}{|c|}{$6 \times 1.19=7.14 \mathrm{cu} \mathrm{m}$} & $6 \times 1.01=6.08 \mathrm{cu} \mathrm{m}$ & $18 \times 0.188=3.39 \mathrm{cu} \mathrm{m}$ \\
\hline & \multicolumn{2}{|c|}{$252 \mathrm{cu} \mathrm{ft}$} & $215 \mathrm{cu} \mathrm{ft}$ & $119 \mathrm{cu} \mathrm{ft}$ \\
\hline \multirow[t]{2}{*}{ Module Weight ${ }^{(4)}$} & \multicolumn{2}{|c|}{$15600 \mathrm{lbm}$} & $15760 \mathrm{lbm}$ & $1390 \mathrm{lbm}$ \\
\hline & \multicolumn{2}{|c|}{$7080 \mathrm{~kg}$} & $7150 \mathrm{~kg}$ & $630 \mathrm{~kg}$ \\
\hline \multirow[t]{2}{*}{ Total IHX Weight ${ }^{(4)}$} & \multicolumn{2}{|c|}{$93600 \mathrm{lbm}$} & $94600 \mathrm{lbm}$ & $25000 \mathrm{lbm}$ \\
\hline & \multicolumn{2}{|c|}{$42500 \mathrm{~kg}$} & $42900 \mathrm{~kg}$ & $11300 \mathrm{~kg}$ \\
\hline \multirow[t]{2}{*}{ Nominal Vessel Dimensions (Diam x Ht) } & \multicolumn{2}{|c|}{$1200 \times 3000 \mathrm{~mm}$} & $1300 \times 2220 \mathrm{~mm}$ & $1730 \times 1140 \mathrm{~mm}$ \\
\hline & \multicolumn{2}{|c|}{$3.9 \times 9.8 \mathrm{ft}$} & $4.3 \times 7.3 \mathrm{ft}$ & $5.7 \times 3.8 \mathrm{ft}$ \\
\hline Estimated Cost ${ }^{(5)}$ & \multicolumn{2}{|c|}{$\mathrm{n} / \mathrm{a}$} & $\$ 5,000,000$ & $\mathrm{n} / \mathrm{a}$ \\
\hline \multicolumn{5}{|l|}{ NOTES: } \\
\hline \multirow[t]{2}{*}{ (1.) A 'module' is defined as follows: } & CORE & $\uparrow$ & & \\
\hline & & $\uparrow_{H}$ & & \\
\hline
\end{tabular}

(2.) Estimates based on our semi-empirical design models

(3.) Design estimates provided by Heatric

(4.) Heatric weight estimates include manifolds. Our estimates are for the core alone.

(5.) Ballpark cost estimate provided by Heatric 

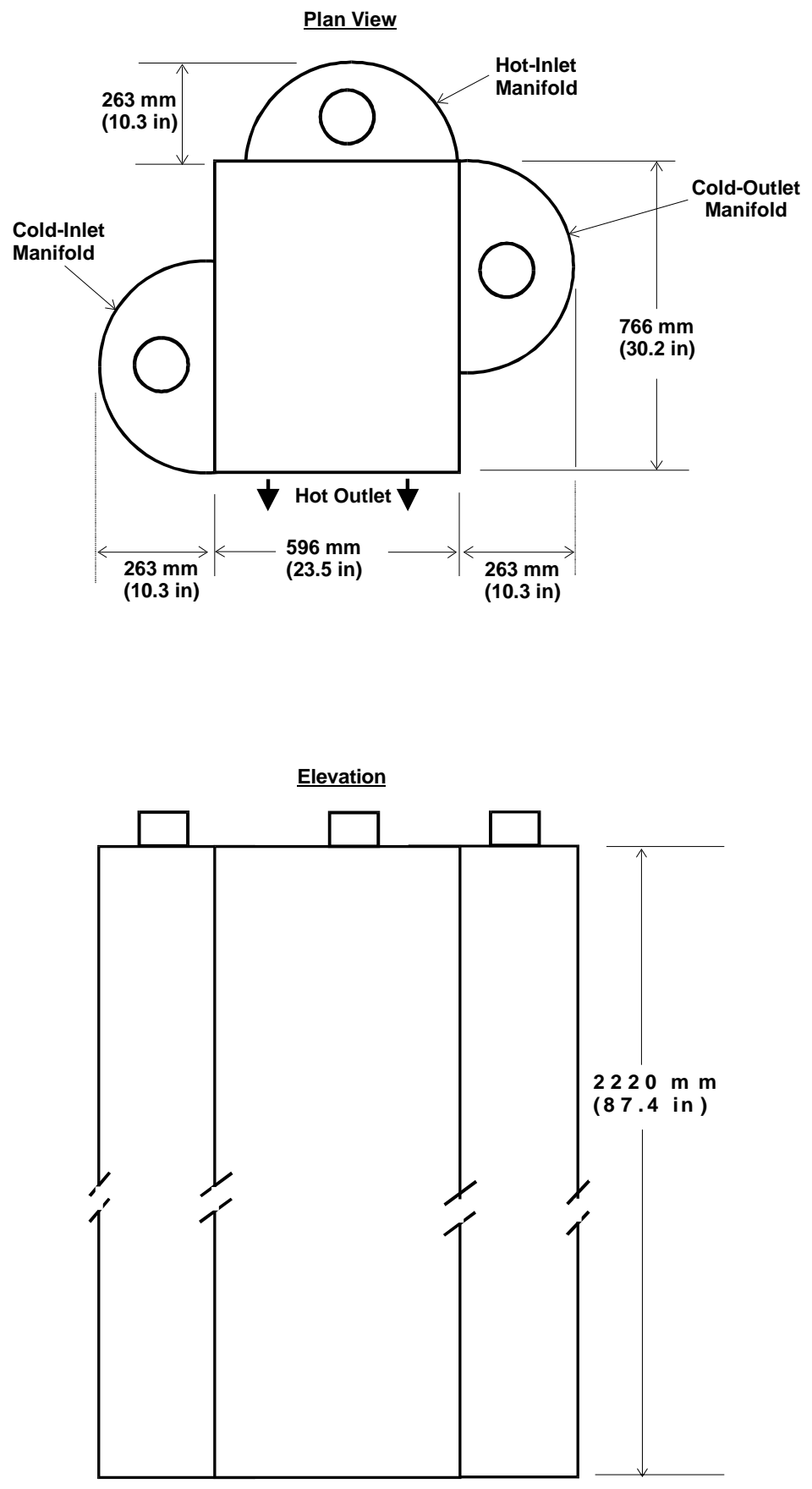

Figure A.1 - Nominal Module Dimensions for Initial Printed Circuit Heat Exchanger IHX Design 


\section{Plan View}

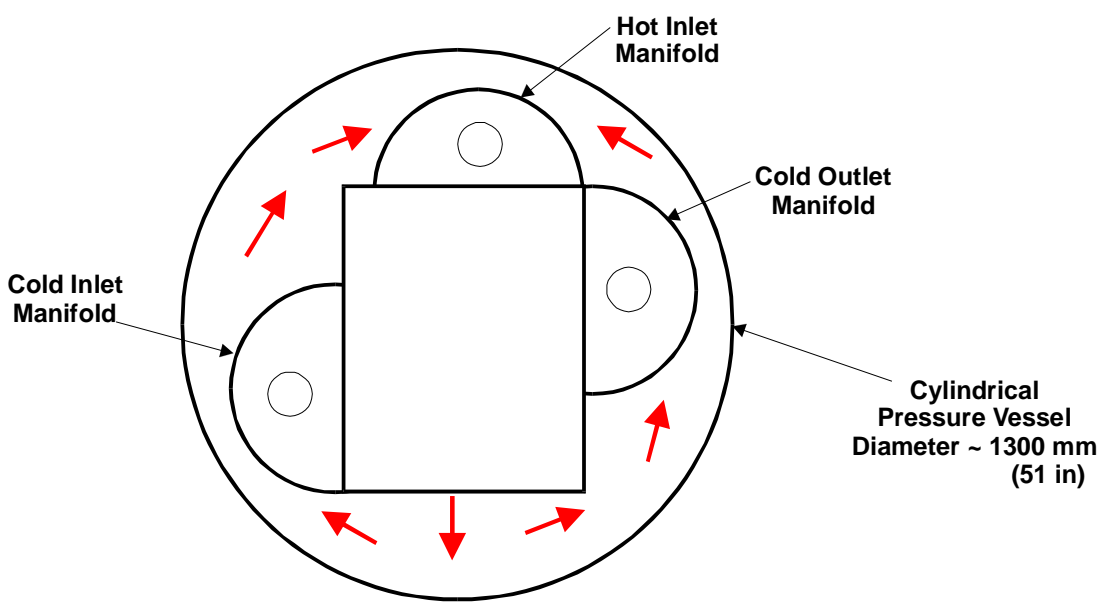

Elevation

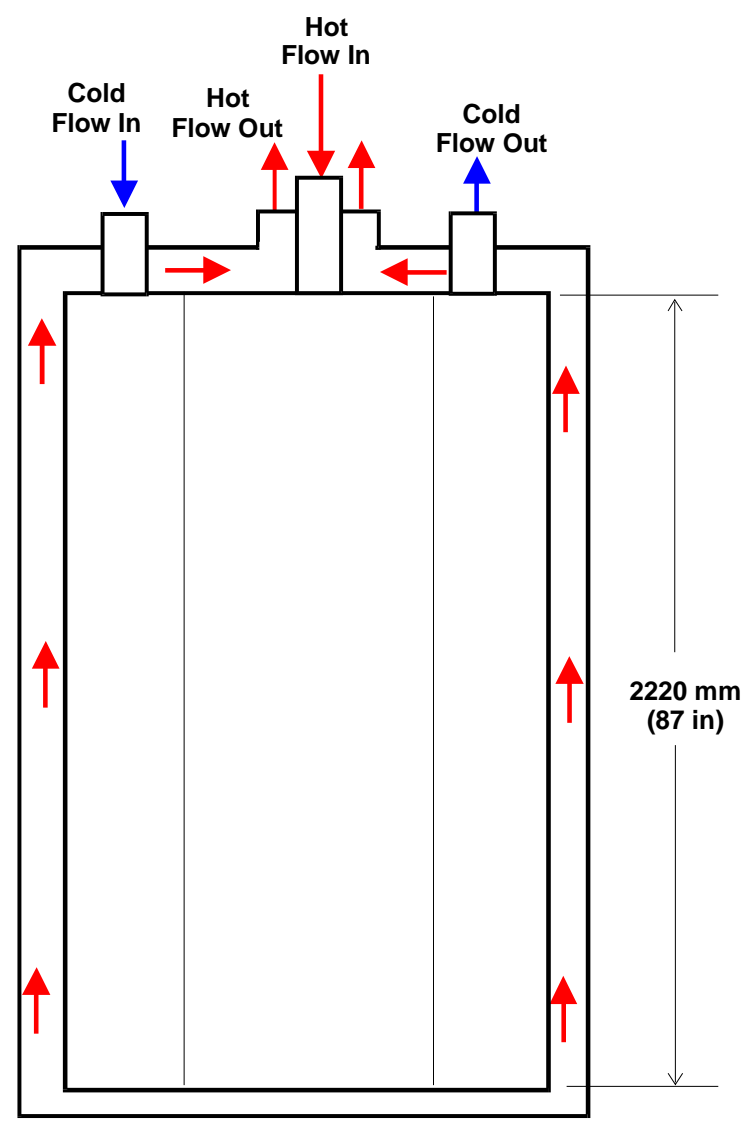

Figure A.2 - Module Packaging for Initial Printed Circuit Heat Exchanger IHX Design 


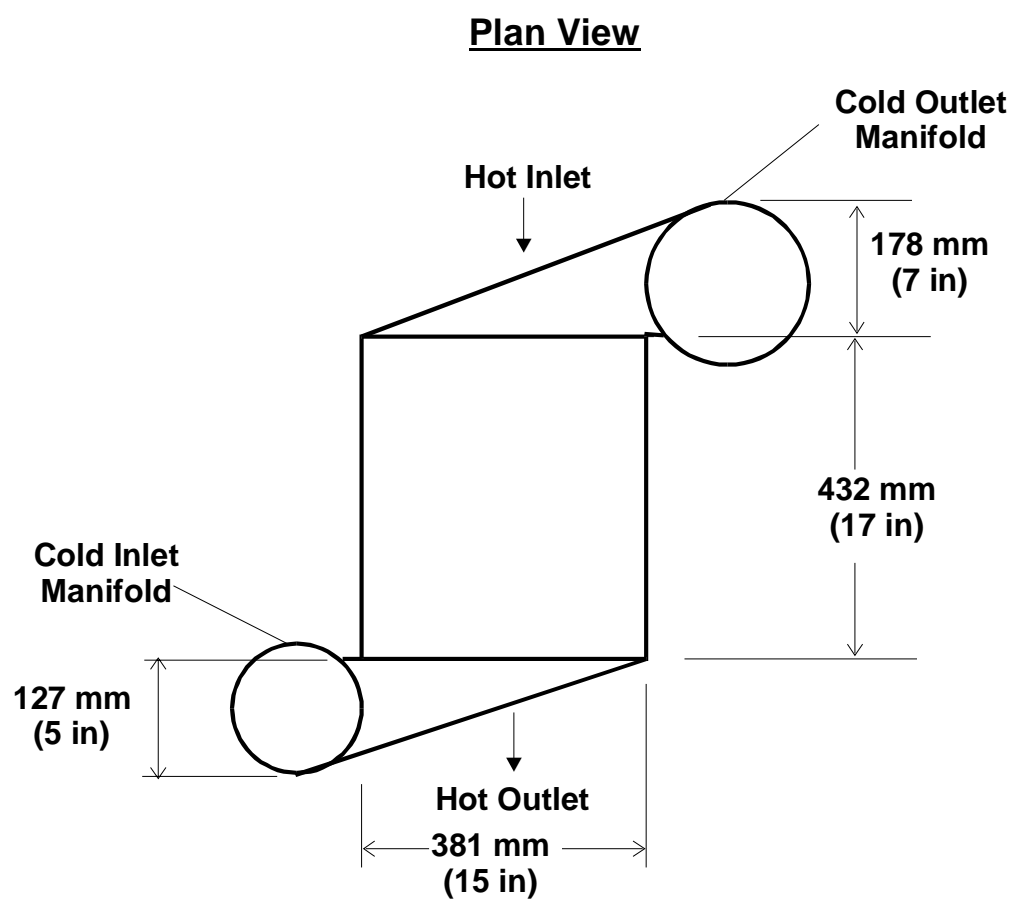

\section{Elevation}

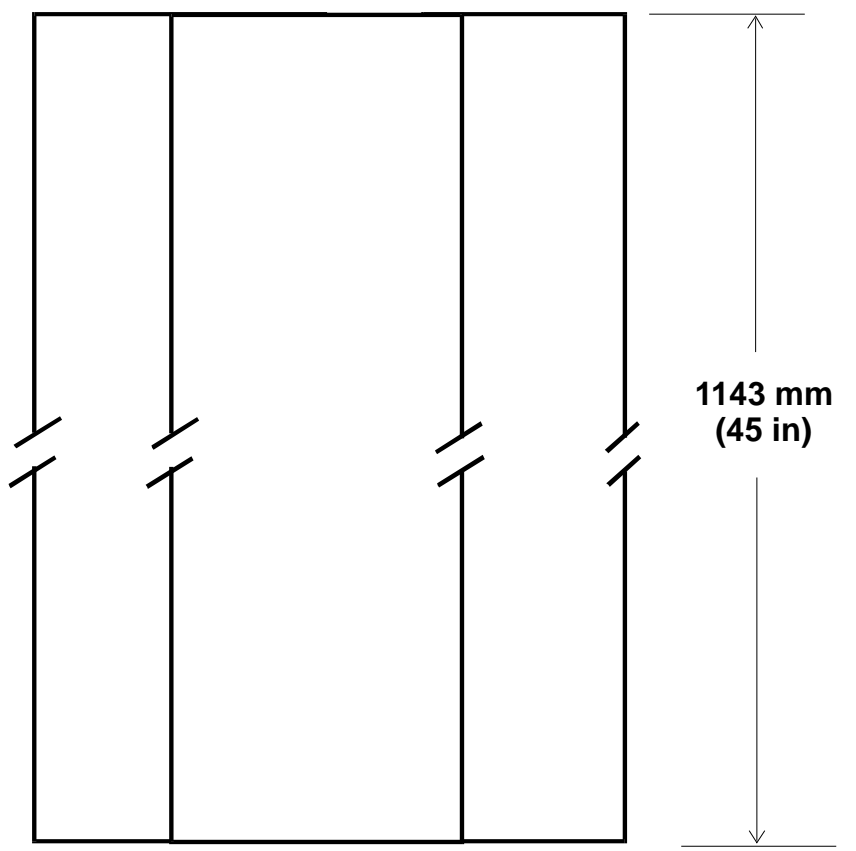

Figure A.3 - Nominal Module Dimensions for Initial Plate Fin Heat Exchanger IHX Design 


\section{Plan View}

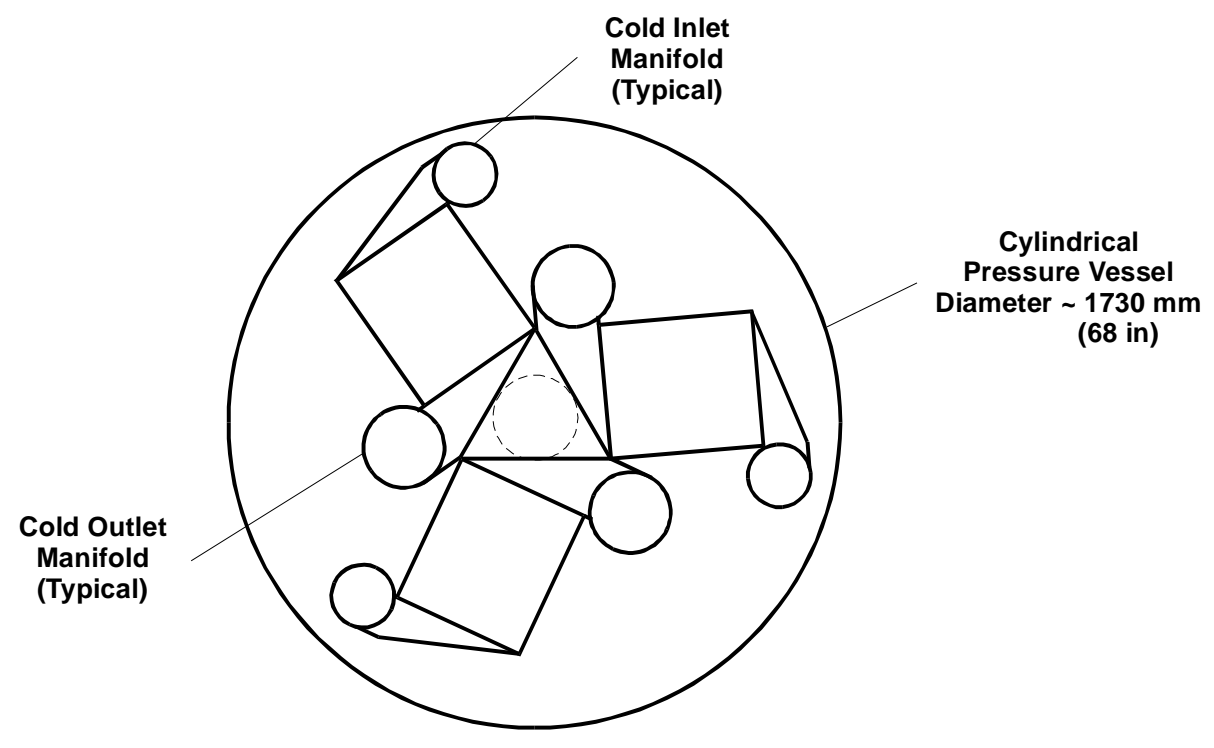

\section{Elevation}

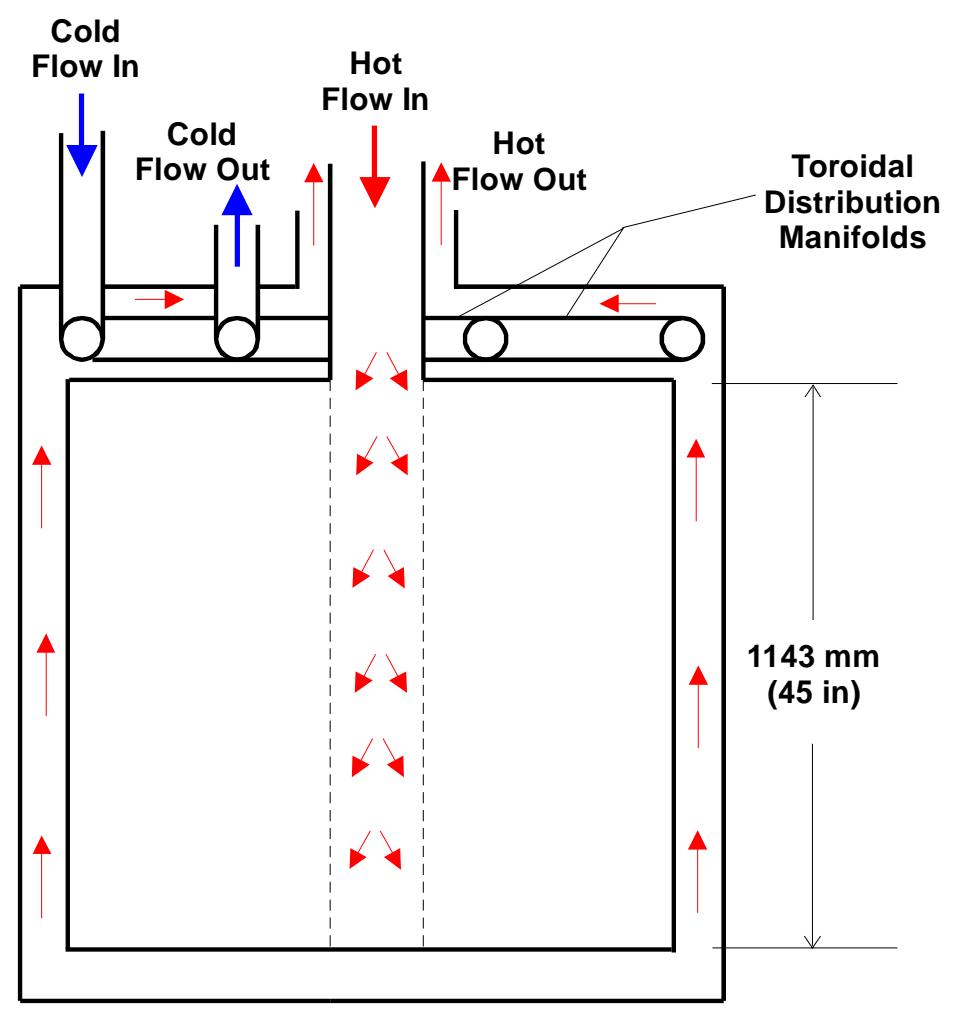

Figure A.4 - Module Packaging for Initial Plate Fin Heat Exchanger IHX Design 
Table A.5 - Additional Parametric Variation of Design Conditions for IHX and Recuperator

\begin{tabular}{|l|c|c|c|c|c|c|}
\cline { 2 - 6 } \multicolumn{1}{c|}{} & \multicolumn{3}{c|}{ IHX } & \multicolumn{3}{c|}{ Recuperator } \\
\hline Effectiveness, \% & 90.0 & 92.5 & 95.0 & 95.0 & 95.0 & 95.0 \\
Allowable Hot-Side Pressure Loss, \% & 2.0 & 2.0 & 2.0 & 0.8 & 1.4 & 2.0 \\
Allowable Cold-Side Pressure Loss, \% & 2.0 & 2.0 & 2.0 & 0.8 & 1.4 & 2.0 \\
\hline Hot Side & & & & & & \\
\hline Flow Rate, kg/sec (Ibm/sec) & $109(240)$ & $109(240)$ & $109(240)$ & $109(240)$ & $109(240)$ & $109(240)$ \\
Inlet Temperature, C (F) & $850(1562)$ & $850(1562)$ & $850(1562)$ & $374(705)$ & $374(705)$ & $374(705)$ \\
Inlet Pressure, MPa (psia) & $7.64(1109)$ & $7.64(1109)$ & $7.64(1109)$ & $1.90(276)$ & $1.90(276)$ & $1.90(276)$ \\
\hline Cold Side & & & & & & \\
Flow Rate, kg/sec (Ibm/sec) & $109(240)$ & $109(240)$ & $109(240)$ & $106(233)$ & $106(233)$ & $106(233)$ \\
Inlet Temperature, C (F) & $350(662)$ & $364(686)$ & $376(709)$ & $104(219)$ & $104(219)$ & $104(219)$ \\
Inlet Pressure, MPa (psia) & $7.90(1146)$ & $7.90(1146)$ & $7.90(1146)$ & $7.99(1160)$ & $7.99(1160)$ & $7.99(1160)$ \\
\hline
\end{tabular}


Table A.6 - PCHE IHX and Recuperator Design Results for Parametric Variations in Design Conditions

\begin{tabular}{|c|c|c|c|c|c|c|}
\hline & \multicolumn{3}{|c|}{ Intermediate Heat Exchanger } & \multicolumn{3}{|c|}{ Recuperator } \\
\hline & \multicolumn{3}{|c|}{ Effectiveness } & \multicolumn{3}{|c|}{ Hot-Side $\Delta \mathrm{P}$} \\
\hline & $90 \%$ & $92.50 \%$ & $95 \%$ & $0.80 \%$ & $1.40 \%$ & $2.00 \%$ \\
\hline Flow Rate, $\mathrm{m}_{\mathrm{c}}$, Ibm/sec & 240 & 240 & 240 & 233 & 233 & 233 \\
\hline Inlet Pressure, $P_{c, \text { in }}$, psia & 1146 & 1146 & 1146 & 1160 & 1160 & 1160 \\
\hline Inlet Temperature, $T_{c, i n}$, deg $F$ & 662.0 & 686.3 & 709.4 & 219.4 & 219.4 & 219.4 \\
\hline Outlet Temperature, $T_{c, \text { out }}$, deg F & 1472.0 & 1496.0 & 1519.0 & 680.6 & 680.6 & 680.6 \\
\hline Flow Rate, $\mathrm{m}_{\mathrm{h}}$, Ibm/sec & 240 & 240 & 240 & 240 & 240 & 240 \\
\hline Inlet Pressure, $P_{h, \text { in }}$, psia & 1109 & 1109 & 1109 & 276 & 276 & 276 \\
\hline Inlet Temperature, $T_{h, i n}$, deg $F$ & 1562.0 & 1562.0 & 1562.0 & 704.8 & 704.8 & 704.8 \\
\hline Outlet Temperature, $T_{h, \text { out }}$, deg $F$ & 752.0 & 752.0 & 751.9 & 257.1 & 257.1 & 257.1 \\
\hline Core Height, in & 655.00 & 712.00 & 816.00 & 3242.00 & 2412.00 & 2000.00 \\
\hline Core Width, in & 23.60 & 23.60 & 23.60 & 23.62 & 23.62 & 23.62 \\
\hline Core Length, in & 15.83 & 20.90 & 30.40 & 15.51 & 17.33 & 18.64 \\
\hline Inlet Header w, in & 9.50 & 9.50 & 9.50 & 5.00 & 5.00 & 5.00 \\
\hline Outlet Header w, in & 9.50 & 9.50 & 9.50 & 5.00 & 5.00 & 5.00 \\
\hline HT Area $A_{c}, \mathrm{ft}^{\wedge} 2$ & 19180 & 27530 & 45880 & 93090 & 77380 & 69020 \\
\hline HT Area $A_{h}, \mathrm{ft}^{\wedge} 2$ & 19180 & 27530 & 45880 & 93090 & 77380 & 69020 \\
\hline Plate Spacing, $b_{c}$, in & 0.03900 & 0.03900 & 0.03900 & 0.03900 & 0.03900 & 0.03900 \\
\hline Channel Dia, $d_{c}$, in & 0.07900 & 0.07900 & 0.07900 & 0.07900 & 0.07900 & 0.07900 \\
\hline Channel Spacing, $S_{p, c}$, in & 0.09600 & 0.09600 & 0.09600 & 0.09600 & 0.09600 & 0.09600 \\
\hline Free Flow/Face Area, $\sigma_{c}$ & 0.402 & 0.402 & 0.402 & 0.402 & 0.402 & 0.402 \\
\hline Surface/Volume, $\beta_{\mathrm{c}}, \mathrm{ft}^{\wedge} 2 / \mathrm{ft}^{\wedge} 3$ & 433.4 & 433.4 & 433.4 & 433.4 & 433.4 & 433.4 \\
\hline Hydraulic Radius, $\mathrm{r}_{\mathrm{H}, \mathrm{c}}, \mathrm{ft}$ & $9.28 \mathrm{E}-04$ & $9.28 \mathrm{E}-04$ & $9.28 \mathrm{E}-04$ & $9.28 \mathrm{E}-04$ & $9.28 \mathrm{E}-04$ & $9.28 \mathrm{E}-04$ \\
\hline Plate Spacing, $b_{h}$, in & 0.03900 & 0.03900 & 0.03900 & 0.03900 & 0.03900 & 0.03900 \\
\hline Channel Dia, $d_{h}$, in & 0.07900 & 0.07900 & 0.07900 & 0.07900 & 0.07900 & 0.07900 \\
\hline Channel Spacing, $S_{p, h}$, in & 0.09600 & 0.09600 & 0.09600 & 0.09600 & 0.09600 & 0.09600 \\
\hline Free Flow/Face Area, $\sigma_{\mathrm{h}}$ & 0.402 & 0.402 & 0.402 & 0.402 & 0.402 & 0.402 \\
\hline Surface/Volume, $\beta_{\mathrm{h}}, \mathrm{ft}^{\wedge} 2 / \mathrm{ft}^{\wedge} 3$ & 433.4 & 433.4 & 433.4 & 433.4 & 433.4 & 433.4 \\
\hline Hydraulic Radius, $\mathrm{r}_{\mathrm{H}, \mathrm{h}}, \mathrm{ft}$ & $9.28 \mathrm{E}-04$ & $9.28 \mathrm{E}-04$ & $9.28 \mathrm{E}-04$ & $9.28 \mathrm{E}-04$ & $9.28 \mathrm{E}-04$ & $9.28 \mathrm{E}-04$ \\
\hline UA, Btu/hr F & 9652000 & 13230000 & 20430000 & 15760000 & 15740000 & 15760000 \\
\hline $\mathrm{Q}, \mathrm{kBtu} / \mathrm{hr}$ & 868600 & 868600 & 868700 & 480100 & 480100 & 480100 \\
\hline Effectiveness \&, \% & 90.0 & 92.5 & 95.0 & 95.0 & 95.0 & 95.0 \\
\hline Reynolds Number, $\operatorname{Re}_{c}$ & 2394.0 & 2178.0 & 1881.0 & 673.1 & 903.4 & 1089.0 \\
\hline HT Coefficient, $h_{c}$, Btu/hr ft^2 F & 1150.0 & 1093.0 & 1003.0 & 349.3 & 424.0 & 479.2 \\
\hline Conductance, $\left(\eta_{0} h A\right)_{c}$, Btu/hr F & 22050000 & 30080000 & 46040000 & 32520000 & 32810000 & 33070000 \\
\hline Cold $\Delta \mathrm{P}_{\mathrm{c}}, \%$ & 1.993 & 2.009 & 2.002 & 0.134 & 0.231 & 0.327 \\
\hline Inlet $\operatorname{Hdr} \Delta \mathrm{P}, \%$ & 0.393 & 0.347 & 0.278 & 0.037 & 0.062 & 0.088 \\
\hline Outlet $\operatorname{Hdr} \Delta \mathrm{P}, \%$ & 0.732 & 0.639 & 0.506 & 0.066 & 0.113 & 0.158 \\
\hline Reynolds Number, $\mathrm{Re}_{\mathrm{h}}$ & 2299.0 & 2115.0 & 1845.0 & 677.8 & 913.0 & 1101.0 \\
\hline HT Coefficient, $h_{h}$, Btu/hr ft^2 F & 1166.0 & 1104.0 & 1010.0 & 359.0 & 435.2 & 491.9 \\
\hline Conductance, $\left(\eta_{\mathrm{o}} \mathrm{hA}\right)_{\mathrm{h}}$, Btu/hr F & 22370000 & 30400000 & 46360000 & 33420000 & 33680000 & 33950000 \\
\hline Hot $\Delta \mathrm{P}_{\mathrm{h}}, \%$ & 1.597 & 1.676 & 1.766 & 0.800 & 1.401 & 2.005 \\
\hline No of Passage Pairs & 5199 & 5652 & 6477 & 25730 & 19150 & 15880 \\
\hline HX Weight, lbm & 84810 & 110600 & 166400 & 340200 & 275600 & 241900 \\
\hline
\end{tabular}

Note: "c" = Cold Side, "h" = Hot Side 
Table A.7 - Summary of Additional IHX and Recuperator Design Results

\begin{tabular}{|c|c|c|c|c|c|c|}
\hline & \multicolumn{3}{|c|}{ Intermediate Heat Exchanger } & \multicolumn{3}{|c|}{ Recuperator } \\
\hline Effectiveness, \% & 90 & 92.5 & 95 & 95 & 95 & 95 \\
\hline Hot-Side Pressure Loss, \% & 1.60 & 1.68 & 1.77 & 0.80 & 1.40 & 2.00 \\
\hline Cold-Side Pressure Loss, \% & 2.00 & 2.00 & 2.00 & 0.13 & 0.23 & 0.33 \\
\hline No. of Modules, $\mathrm{N}$ & 6 & 6 & 6 & 30 & 30 & 30 \\
\hline Module Width, W, in & 23.62 & 23.62 & 23.62 & 23.62 & 23.62 & 23.62 \\
\hline Module Length, $L$, in & 34.83 & 39.9 & 49.4 & 25.51 & 27.33 & 28.64 \\
\hline Module Height, $\mathrm{H}$, in & 109.2 & 118.7 & 136.0 & 108.1 & 80.4 & 66.7 \\
\hline Module Width, W, mm & 600 & 600 & 600 & 600 & 600 & 600 \\
\hline Module Length, L, mm & 885 & 1013 & 1255 & 648 & 694 & 727 \\
\hline Module Height, $\mathrm{H}, \mathrm{mm}$ & 2773 & 3014 & 3454 & 2745 & 2042 & 1693 \\
\hline Est. Total HX Weight, Ibm & 85,658 & 111,706 & 168,064 & 343,602 & 278,356 & 244,319 \\
\hline Estimated HX Cost, US 2001 \$M & 4.5 & 5.9 & 8.9 & 2.6 & 2.1 & 1.9 \\
\hline PLATE-FIN HX ESTIMATES & & & & & & \\
\hline Approximate Total HX Weight, Ibm & 22,785 & 29,714 & 44,705 & 91,398 & 74,043 & 64,989 \\
\hline Approximate HX Cost, US 2001 \$M & 1.2 & 1.6 & 2.4 & 0.7 & 0.6 & 0.5 \\
\hline
\end{tabular}

NOTES:

(1.) A 'module' is defined as follows:

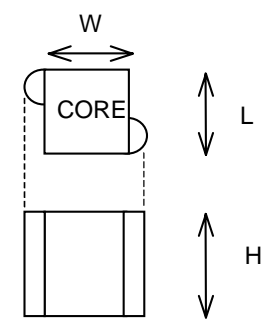

(2.) Costs assumed proportional to weight

(3.) Estimated costs are based on scaling Heatric quote for Incoloy $800 \mathrm{IHX}$

(4.) Costs for recuperator assume 347 SS material and unit cost $(\$ / \mathrm{lbm}) 1 / 7$ th Incoloy cost

(5.) Plate-fin estimates are approximate based on scaling original PFHE/PCHE weight comparisons. 


\section{Appendix B Concepts-NREC Turbomachinery Design}

The preliminary designs for the compressors and the turbines for the MPBR cycle has been conducted by Concepts-NREC. For compressors, both centrifugal and axi-centrifugal types were investigated. For turbines, the axial type was adopted.

The turbomachinery design requirements are listed in Table B.1. Tables B.2 to B.4 tabulate the centrifugal compressor designs applied to the four-shaft arrangement and three-shaft arrangement, respectively. The Five-stage centrifugal performance is demonstrated in Table B.5 and Figure B.1 to B.4. The size and cost for the axi-centrifugal compressor designs are listed in the Table B.6 and Table B.7, respectively. Figures B.5 to B.8 show the characteristics of the axi-centrifugal compressor. Its meridional view is shown in Figure B.9. For the HP turbine and LP turbine, the size and cost are listed in Tables B.8 and B.9. Figure B.10 and B.11 show its characteristics. The power turbine size and cost are listed in Tables B.10 and B.11. Table B.12 lists the detailed geometry of the five-stage centrifugal compressor.

Table B.1 - Turbomachinery Design Requirements

\begin{tabular}{|c|c|c|c|}
\hline Design Requirements & Year 1 & Year 2 & Notes \\
\hline Working Fluid & Helium & - & \\
\hline Output Power Generated & $110 \mathrm{MW}$ & $120 \mathrm{MW}$ & \\
\hline Target Cycle Efficiency & $>40 \%$ & $>45 \%$ & Busbar Eff. \\
\hline Flow Rate & $118.9 \mathrm{~kg} / \mathrm{sec}$ & $126.7 \mathrm{~kg} / \mathrm{sec}$ & \\
\hline Minimum Fluid Pressure & $2.0 \mathrm{MPa}$ & $2.71 \mathrm{MPa}$ & \\
\hline Maximum Fluid Temperature & $800.0^{\circ} \mathrm{C}$ & $879.4^{\circ} \mathrm{C}$ & \\
\hline Overall Cycle Pressure Ratio & $\sim 4.0: 1$ & $\sim 3.0: 1$ & \\
\hline Ancillary Requirements & & & \\
\hline Inter-device Pipe Velocity & $120 \mathrm{~m} / \mathrm{s}$ & - & \\
\hline Life expectancy & $30-50$ years & - & \\
\hline
\end{tabular}

Table B.2 - Numbers and Sizes of Centrifugal Compressors

\begin{tabular}{|c|c|c|c|c|}
\hline $\begin{array}{c}\text { Number of } \\
\text { Compressors }\end{array}$ & $\begin{array}{c}\text { Stages per } \\
\text { Compressor }\end{array}$ & $\begin{array}{c}\text { Optimal } \\
\text { Shaft Speed }\end{array}$ & $\begin{array}{c}\text { Casing Inner } \\
\text { Diameter }\end{array}$ & $\begin{array}{c}\text { Compressor } \\
\text { Casing Length }\end{array}$ \\
\hline 3 & 6 & $7500 \mathrm{rpm}$ & $1.62 \mathrm{~m}$ & $3.4 \mathrm{~m}$ \\
\hline 4 & 5 & $5900 \mathrm{rpm}$ & $1.74 \mathrm{~m}$ & $3.3 \mathrm{~m}$ \\
\hline
\end{tabular}


Table B.3 - 3-Compressor (Centrifugal) Arrangement Costs

\begin{tabular}{|c|c|c|c|}
\hline & $\begin{array}{c}\text { Recurring } \\
\text { Cost (\$US) }\end{array}$ & $\begin{array}{c}\text { Development/ } \\
\text { Non-recurring } \\
\text { Cost (\$US) }\end{array}$ & Total (\$US) \\
\hline $1^{\text {st }}$ Compressor & 2.9 & 2.1 & 5.0 \\
\hline $2^{\text {nd }}$ Compressor & 2.3 & - & 2.3 \\
\hline $3^{\text {rd }}$ Compressor & 2.3 & - & 2.3 \\
\hline
\end{tabular}

The estimated costs in millions of 2001 \$US.

Table B.4 - 4-Compressor (Centrifugal) Arrangement Costs

\begin{tabular}{|c|c|c|c|}
\hline & Cost (\$US) & $\begin{array}{c}\text { Development/ } \\
\text { Non-recurring } \\
\text { Cost (\$US) }\end{array}$ & Total (\$US) \\
\hline $1^{\text {st }}$ Compressor & 2.6 & 2.1 & 4.7 \\
\hline $2^{\text {nd }}$ Compressor & 1.8 & - & 1.8 \\
\hline $3^{\text {rd }}$ Compressor & 1.8 & - & 1.8 \\
\hline $4^{\text {th }}$ Compressor & 1.8 & - & 1.8 \\
\hline
\end{tabular}

The estimated costs in millions of 2001 \$US. 
Table B.5 - Five-Stage Centrifugal Compressor Design Data

\begin{tabular}{|c|c|c|c|c|c|c|c|}
\hline Design & \multirow{2}{*}{$\begin{array}{l}\text { Inlet Stag. Pressure } \\
\text { Inlet Stag. Temperature }\end{array}$} & \multicolumn{6}{|c|}{$2074.098 \mathrm{kPa}$} \\
\hline & & \multicolumn{6}{|l|}{$311.9 \mathrm{~K}$} \\
\hline & $\begin{array}{l}\text { Rotational speed } \\
\text { Mass flow }\end{array}$ & \multicolumn{6}{|l|}{$5900 \mathrm{rpm}$} \\
\hline & Mass flow & \multicolumn{6}{|c|}{$136.42 \mathrm{~kg} / \mathrm{s}$} \\
\hline & Rel. flow coefficient & \multicolumn{6}{|l|}{0.755} \\
\hline & Pressure ratio & \multicolumn{6}{|l|}{1.566} \\
\hline & Temperature ratio & \multicolumn{6}{|l|}{1.231} \\
\hline & Effic. (w/o IGV losses) & \multicolumn{6}{|c|}{86.054 percent } \\
\hline & Required power & \multicolumn{6}{|c|}{$51048.47 \mathrm{~kW}$} \\
\hline & Effic. (with IGV losses) & \multicolumn{6}{|c|}{86.054 percent } \\
\hline \multicolumn{8}{|c|}{ Off-Design Performance } \\
\hline $\begin{array}{l}\text { Speed } \\
\text { ratio }\end{array}$ & Rel. Flow Coefficient & $\begin{array}{l}\text { Mass } \\
\text { flow } \\
(\mathrm{kg} / \mathrm{s})\end{array}$ & $\begin{array}{l}\text { Press- } \\
\text { ure } \\
\text { ratio }\end{array}$ & $\begin{array}{l}\text { Tempera- } \\
\text { ture ratio }\end{array}$ & $\begin{array}{l}\text { Efficiency } \\
\text { w/o IGV } \\
\text { Losses }\end{array}$ & $\begin{array}{l}\text { Power } \\
\text { required }\end{array}$ & $\begin{array}{l}\text { Effic. } \\
\text { with } \\
\text { IGV } \\
\text { losses } \\
\end{array}$ \\
\hline 1 & 0.441 & 80.488 & 1.616 & 1.272 & 79.59 & 35408.45 & 79.59 \\
\hline 1 & 0.5 & 91.538 & 1.615 & 1.264 & 81.485 & 39165.38 & 81.485 \\
\hline 1 & 0.56 & 102.588 & 1.609 & 1.256 & 83.079 & 42605.82 & 83.079 \\
\hline 1 & 0.622 & 113.638 & 1.599 & 1.248 & 84.376 & 45718.61 & 84.376 \\
\hline 1 & 0.685 & 124.688 & 1.585 & 1.24 & 85.364 & 48492.34 & 85.364 \\
\hline 1 & 0.751 & 135.738 & 1.568 & 1.232 & 86.053 & 50913.43 & 86.053 \\
\hline 1 & 0.819 & 146.788 & 1.544 & 1.223 & 86.111 & 52929.28 & 86.111 \\
\hline 1 & 0.892 & 157.838 & 1.514 & 1.213 & 85.551 & 54543.84 & 85.551 \\
\hline 1 & 0.968 & 168.888 & 1.481 & 1.204 & 84.382 & 55736.92 & 84.382 \\
\hline 1 & 1.049 & 179.938 & 1.445 & 1.194 & 82.857 & 56503.89 & 82.857 \\
\hline 1 & 80.8 & 190.988 & 1.408 & 1.184 & 80.852 & 56814.26 & 80.852 \\
\hline $\begin{array}{l}\text { Speed } \\
\text { ratio }\end{array}$ & Rel. Flow Coefficient & $\begin{array}{l}\text { Mass } \\
\text { flow } \\
(\mathrm{kg} / \mathrm{s})\end{array}$ & $\begin{array}{l}\text { Press- } \\
\text { ure } \\
\text { ratio }\end{array}$ & $\begin{array}{l}\text { Tempera- } \\
\text { ture ratio }\end{array}$ & $\begin{array}{l}\text { Efficiency } \\
\text { w/o IGV } \\
\text { Losses }\end{array}$ & $\begin{array}{l}\text { Power } \\
\text { required }\end{array}$ & $\begin{array}{l}\text { Effic. } \\
\text { with } \\
\text { IGV } \\
\text { losses } \\
\end{array}$ \\
\hline 0.95 & 0.429 & 76.395 & 1.549 & 1.245 & 79.709 & 30261.17 & 79.709 \\
\hline 0.95 & 0.488 & 86.9 & 1.548 & 1.238 & 81.609 & 33458 & 81.609 \\
\hline 0.95 & 0.547 & 97.404 & 1.542 & 1.231 & 83.198 & 36379.39 & 83.198 \\
\hline 0.95 & 0.607 & 107.908 & 1.532 & 1.223 & 84.48 & 39016.39 & 84.48 \\
\hline 0.95 & 0.668 & 118.413 & 1.519 & 1.216 & 85.442 & 41359.7 & 85.442 \\
\hline 0.95 & 0.732 & 128.917 & 1.503 & 1.208 & 86.058 & 43396.9 & 86.058 \\
\hline 0.95 & 0.798 & 139.421 & 1.481 & 1.2 & 86.099 & 45091.83 & 86.099 \\
\hline 0.95 & 0.869 & 149.926 & 1.455 & 1.191 & 85.406 & 46440.43 & 85.406 \\
\hline 0.95 & 0.943 & 160.43 & 1.425 & 1.183 & 84.185 & 47435.35 & 84.185 \\
\hline 0.95 & 1.019 & 170.934 & 1.393 & 1.174 & 82.601 & 48069.4 & 82.601 \\
\hline 0.95 & 1.1 & 181.439 & 1.36 & 1.164 & 80.53 & 48318.99 & 80.53 \\
\hline
\end{tabular}


Table B.5 - Five-Stage Centrifugal Compressor Design Data (cont's)

\begin{tabular}{|c|c|c|c|c|c|c|c|}
\hline $\begin{array}{l}\text { Speed } \\
\text { ratio }\end{array}$ & Rel. Flow Coefficient & $\begin{array}{l}\text { Mass } \\
\text { flow } \\
(\mathrm{kg} / \mathrm{s})\end{array}$ & $\begin{array}{l}\text { Press- } \\
\text { ure } \\
\text { ratio }\end{array}$ & $\begin{array}{l}\text { Tempera- } \\
\text { ture ratio }\end{array}$ & $\begin{array}{l}\text { Efficiency } \\
\text { w/o IGV } \\
\text { Losses }\end{array}$ & $\begin{array}{l}\text { Power } \\
\text { required }\end{array}$ & $\begin{array}{l}\text { Effic. } \\
\text { with } \\
\text { IGV } \\
\text { losses }\end{array}$ \\
\hline 0.90 & 0.409 & 70.938 & 1.487 & 1.22 & 79.533 & 25259.27 & 79.533 \\
\hline 0.90 & 0.466 & 81.033 & 1.485 & 1.214 & 81.506 & 28025.5 & 81.506 \\
\hline 0.90 & 0.525 & 91.129 & 1.48 & 1.207 & 83.149 & 30548.52 & 83.149 \\
\hline 0.90 & 0.584 & 101.224 & 1.472 & 1.2 & 84.466 & 32820.93 & 84.466 \\
\hline 0.90 & 0.644 & 111.319 & 1.46 & 1.193 & 85.446 & 34835.31 & 85.446 \\
\hline 0.90 & 0.707 & 121.414 & 1.445 & 1.186 & 86.034 & 36580.57 & 86.034 \\
\hline 0.90 & 0.772 & 131.509 & 1.425 & 1.179 & 86.069 & 38031.64 & 86.069 \\
\hline 0.90 & 0.84 & 141.604 & 1.401 & 1.171 & 85.301 & 39181.53 & 85.301 \\
\hline 0.90 & 0.912 & 151.699 & 1.374 & 1.163 & 84.025 & 40027.38 & 84.025 \\
\hline 0.90 & 0.986 & 161.794 & 1.346 & 1.155 & 82.371 & 40562.32 & 82.371 \\
\hline 0.90 & 1.063 & 171.889 & 1.316 & 1.146 & 80.209 & 40767.25 & 80.209 \\
\hline $\begin{array}{l}\text { Speed } \\
\text { ratio }\end{array}$ & Rel. Flow Coefficient & $\begin{array}{l}\text { Mass } \\
\text { flow } \\
(\mathrm{kg} / \mathrm{s})\end{array}$ & $\begin{array}{l}\text { Press- } \\
\text { ure } \\
\text { ratio }\end{array}$ & $\begin{array}{l}\text { Tempera- } \\
\text { ture ratio }\end{array}$ & $\begin{array}{l}\text { Efficiency } \\
\text { w/o IGV } \\
\text { Losses }\end{array}$ & $\begin{array}{l}\text { Power } \\
\text { required }\end{array}$ & $\begin{array}{l}\text { Effic. } \\
\text { with } \\
\text { IGV } \\
\text { losses }\end{array}$ \\
\hline 0.85 & 0.387 & 65.482 & 1.429 & 1.197 & 79.305 & 20843.61 & 79.305 \\
\hline 0.85 & 0.443 & 75.167 & 1.428 & 1.191 & 81.363 & 23223.03 & 81.363 \\
\hline 0.85 & 0.501 & 84.853 & 1.423 & 1.185 & 83.069 & 25389.2 & 83.069 \\
\hline 0.85 & 0.559 & 94.539 & 1.416 & 1.179 & 84.429 & 27336.13 & 84.429 \\
\hline 0.85 & 0.618 & 104.225 & 1.405 & 1.172 & 85.434 & 29057.9 & 85.434 \\
\hline 0.85 & 0.679 & 113.911 & 1.391 & 1.166 & 86.006 & 30544.75 & 86.006 \\
\hline 0.85 & 0.742 & 123.597 & 1.374 & 1.159 & 86.066 & 31784.32 & 86.066 \\
\hline 0.85 & 0.809 & 133.282 & 1.352 & 1.152 & 85.173 & 32755.65 & 85.173 \\
\hline 0.85 & 0.878 & 142.968 & 1.328 & 1.145 & 83.872 & 33472.47 & 83.872 \\
\hline 0.85 & 0.949 & 152.654 & 1.303 & 1.137 & 82.147 & 33922.36 & 82.147 \\
\hline 0.85 & 1.023 & 162.34 & 1.277 & 1.13 & 79.891 & 34091.25 & 79.891 \\
\hline $\begin{array}{l}\text { Speed } \\
\text { ratio }\end{array}$ & Rel. Flow Coefficient & $\begin{array}{l}\text { Mass } \\
\text { flow } \\
(\mathrm{kg} / \mathrm{s})\end{array}$ & $\begin{array}{l}\text { Press- } \\
\text { ure } \\
\text { ratio }\end{array}$ & $\begin{array}{l}\text { Tempera- } \\
\text { ture ratio }\end{array}$ & $\begin{array}{l}\text { Efficiency } \\
\text { w/o IGV } \\
\text { Losses }\end{array}$ & $\begin{array}{l}\text { Power } \\
\text { required }\end{array}$ & $\begin{array}{l}\text { Effic. } \\
\text { with } \\
\text { IGV } \\
\text { losses }\end{array}$ \\
\hline 0.5 & 0.259 & 38.198 & 1.139 & 1.067 & 79.753 & 4158.666 & 79.753 \\
\hline 0.5 & 0.297 & 43.791 & 1.138 & 1.065 & 81.794 & 4614.844 & 81.794 \\
\hline 0.5 & 0.335 & 49.384 & 1.136 & 1.063 & 83.439 & 5026.47 & 83.439 \\
\hline 0.5 & 0.373 & 54.977 & 1.133 & 1.061 & 84.687 & 5393.121 & 84.687 \\
\hline 0.5 & 0.412 & 60.57 & 1.129 & 1.058 & 85.506 & 5714.344 & 85.506 \\
\hline 0.5 & 0.451 & 66.164 & 1.124 & 1.056 & 85.824 & 5989.362 & 85.824 \\
\hline 0.5 & 0.491 & 71.757 & 1.118 & 1.054 & 85.503 & 6218.171 & 85.503 \\
\hline 0.5 & 0.531 & 77.35 & 1.111 & 1.051 & 84.369 & 6399.457 & 84.369 \\
\hline 0.5 & 0.572 & 82.943 & 1.104 & 1.049 & 82.886 & 6534.759 & 82.886 \\
\hline 0.5 & 0.613 & 88.537 & 1.096 & 1.046 & 80.979 & 6623.057 & 80.979 \\
\hline 0.5 & 0.655 & 94.13 & 1.088 & 1.044 & 78.557 & 6663.258 & 78.557 \\
\hline
\end{tabular}


Five-stage centrifugal compressor characteristics

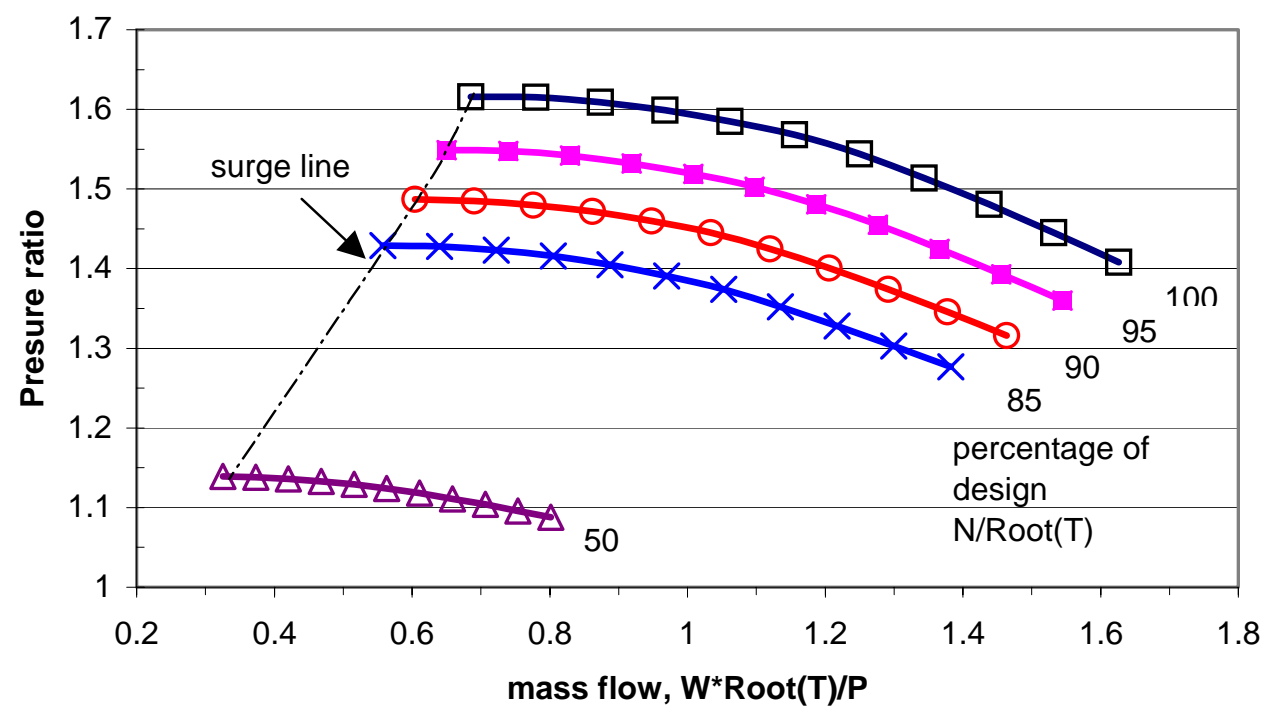

Figure B.1 - Estimated Pressure Ratio Versus Corrected Flow for a Five-Stage Centrifugal Compressor

Five-stage centrifugal compressor characteristics

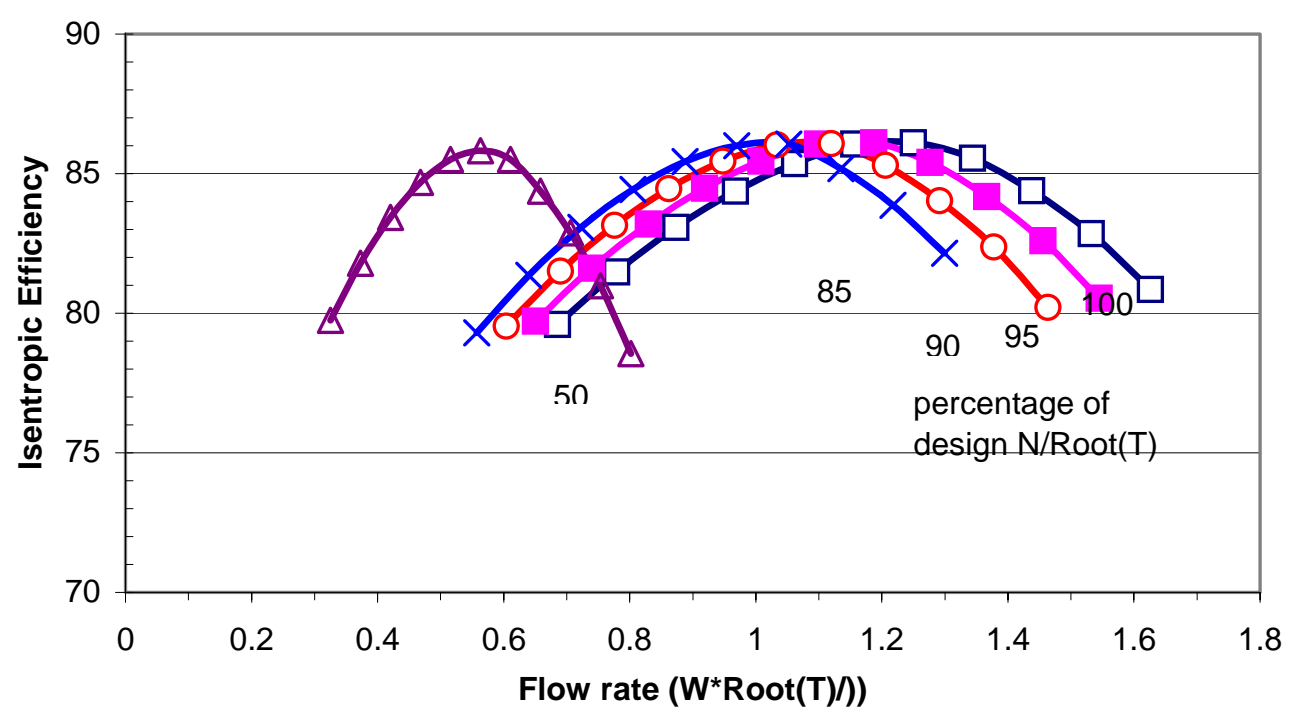

Figure B.2 - Estimated Isentropic Efficiency Versus Corrected Flow for a Five-Stage Centrifugal Compressor 
ESTIMATED 5-STAGE CENTRIFUGAL COMPRESSOR CHARACTERIZAION

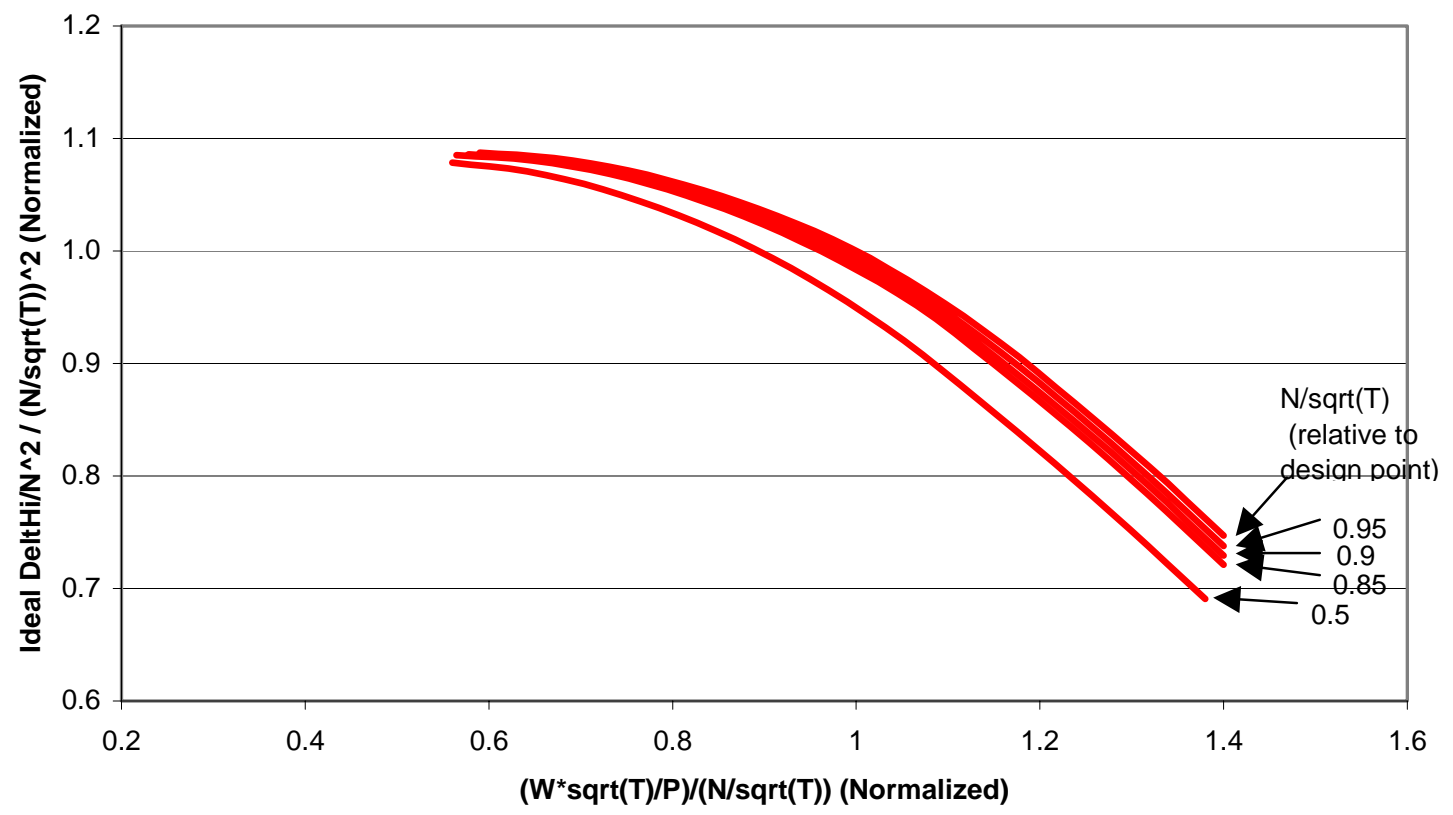

Figure B.3 - Estimated Pressure Coefficient Versus Corrected Flow Normalized to FiveStage Compressor Design Point

ESTIMATED 5-STAGE CENTRIFUGAL COMPRESSOR CHARACTERIZAION

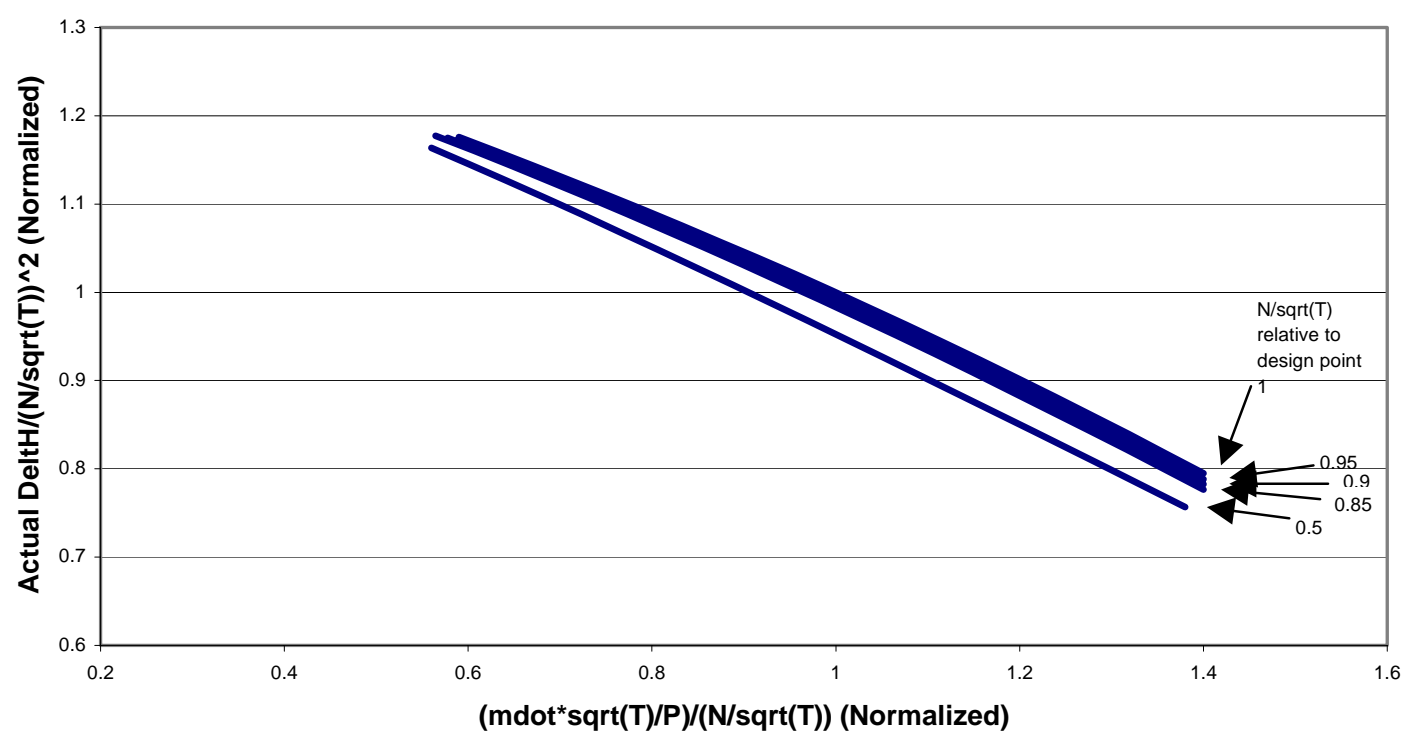

Figure B.4 - Estimated Work Coefficient Versus Corrected Flow Normalized to Five-Stage Compressor Design Point 
Table B.6 - Numbers and Sizes of Axi-centrifugal Compressors

\begin{tabular}{|c|c|c|c|c|}
\hline $\begin{array}{c}\text { Number of } \\
\text { Compressors }\end{array}$ & $\begin{array}{c}\text { Stages per } \\
\text { Compressor }\end{array}$ & $\begin{array}{c}\text { Optimal } \\
\text { Shaft Speed }\end{array}$ & $\begin{array}{c}\text { Casing Inner } \\
\text { Diameter }\end{array}$ & $\begin{array}{c}\text { Compressor } \\
\text { Casing Length }\end{array}$ \\
\hline 4 & $8+1$ & $8000 \mathrm{rpm}$ & $1.0 \mathrm{~m} / 2.6 \mathrm{~m}$ & $3.0 \mathrm{~m}$ \\
\hline
\end{tabular}

Table B.7 -- Four- Compressor (Axi-centrifugal) Costs

\begin{tabular}{|c|c|c|c|}
\hline & Cost (\$US) & $\begin{array}{c}\text { Development/ } \\
\text { Non-recurring Cost }\end{array}$ & Total (\$US) \\
\hline $1^{\text {st }}$ Compressor & 3.3 & 10.2 & 13.5 \\
\hline $2^{\text {nd }}$ Compressor & 2.3 & - & 2.3 \\
\hline $3^{\text {rd }}$ Compressor & 2.3 & - & 2.3 \\
\hline $4^{\text {th }}$ Compressor & 2.3 & - & 2.3 \\
\hline
\end{tabular}

The estimated costs in millions of 2001 \$US.

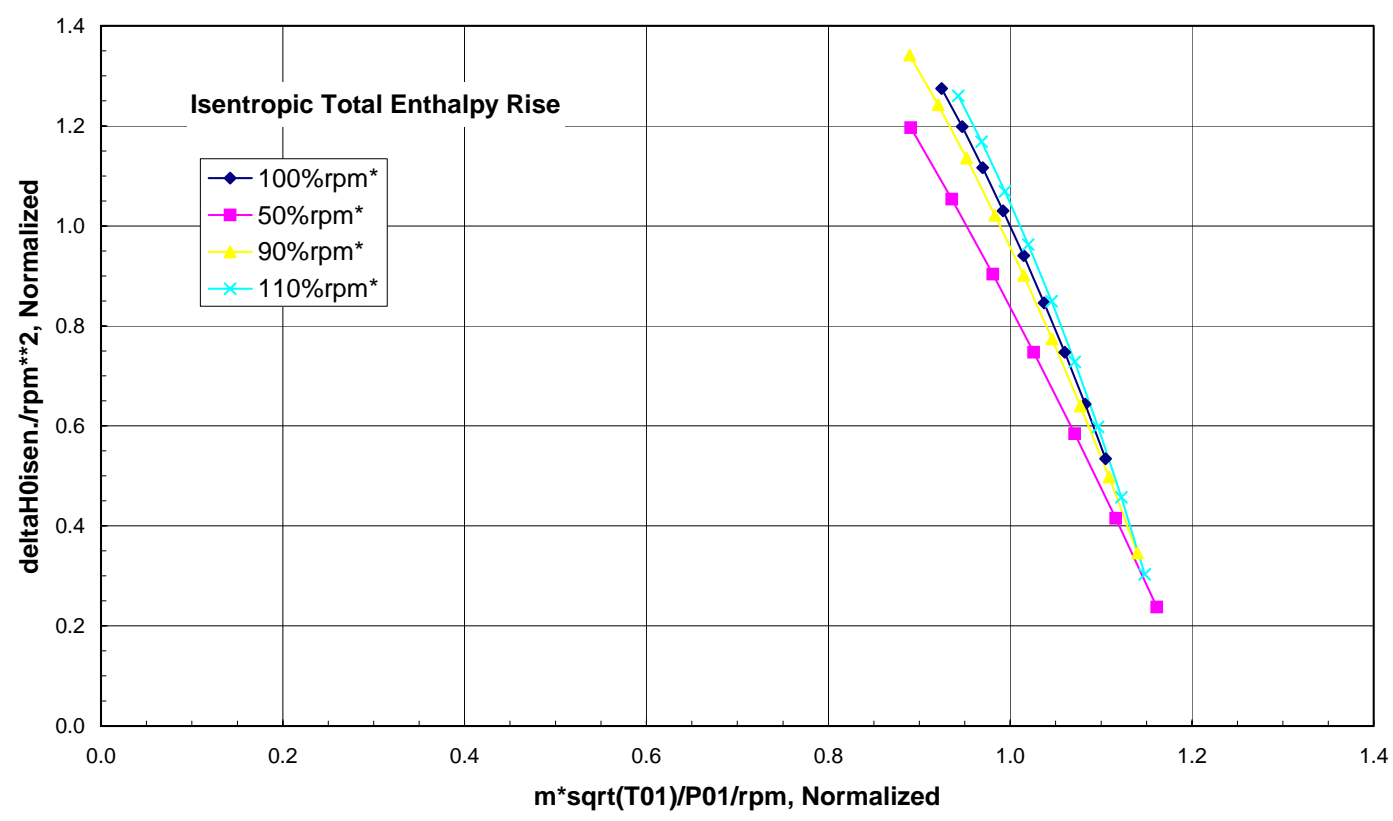

Figure B.5 - Estimated Pressure Coefficient Versus Corrected Flow Normalized to an 8+1 Axi-Centrifugal Compressor Design Point 


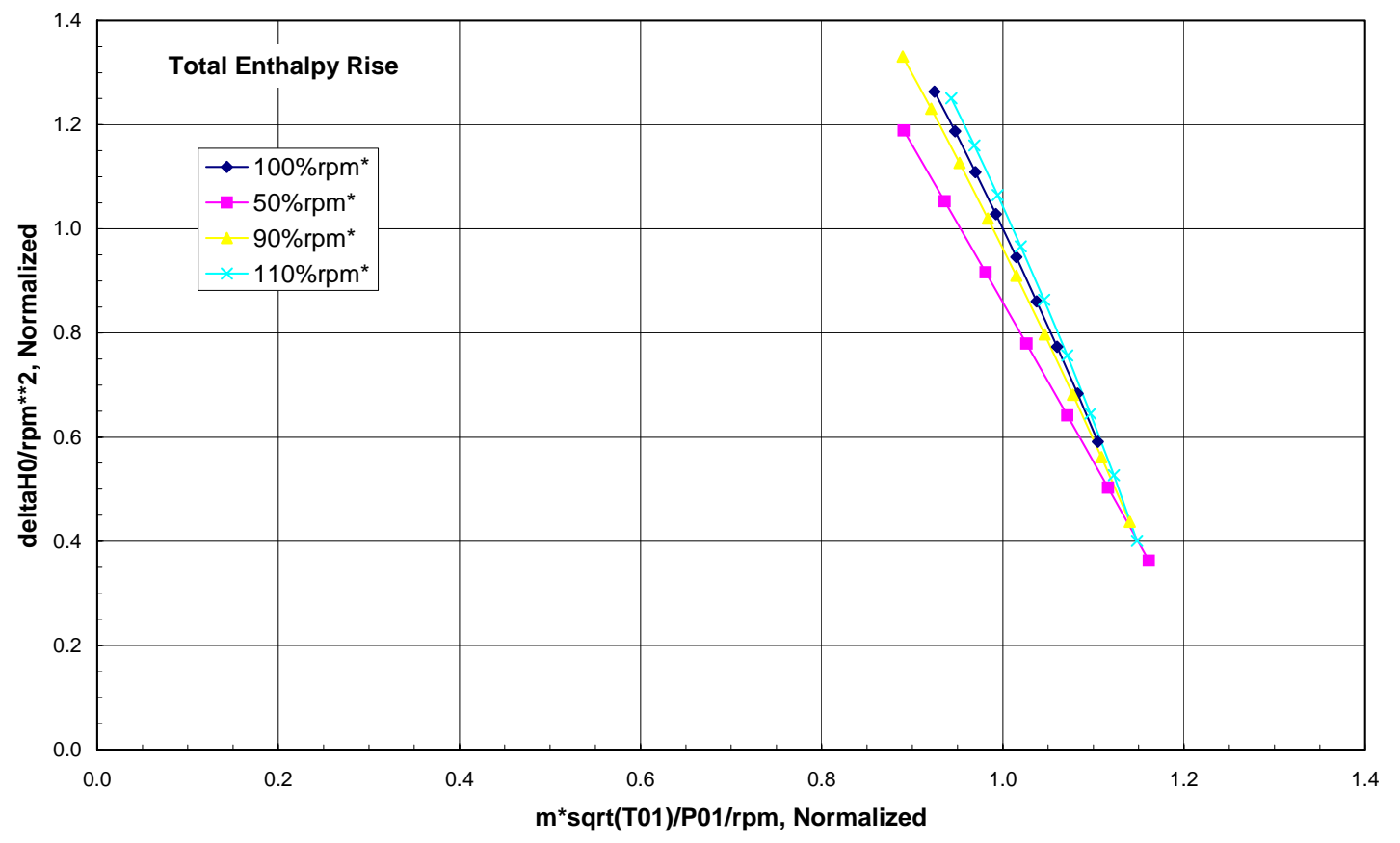

Figure B.6 - Estimated Work Coefficient Versus Corrected Flow Normalized to an $8+1$ Axi-Centrifugal Compressor Design Point

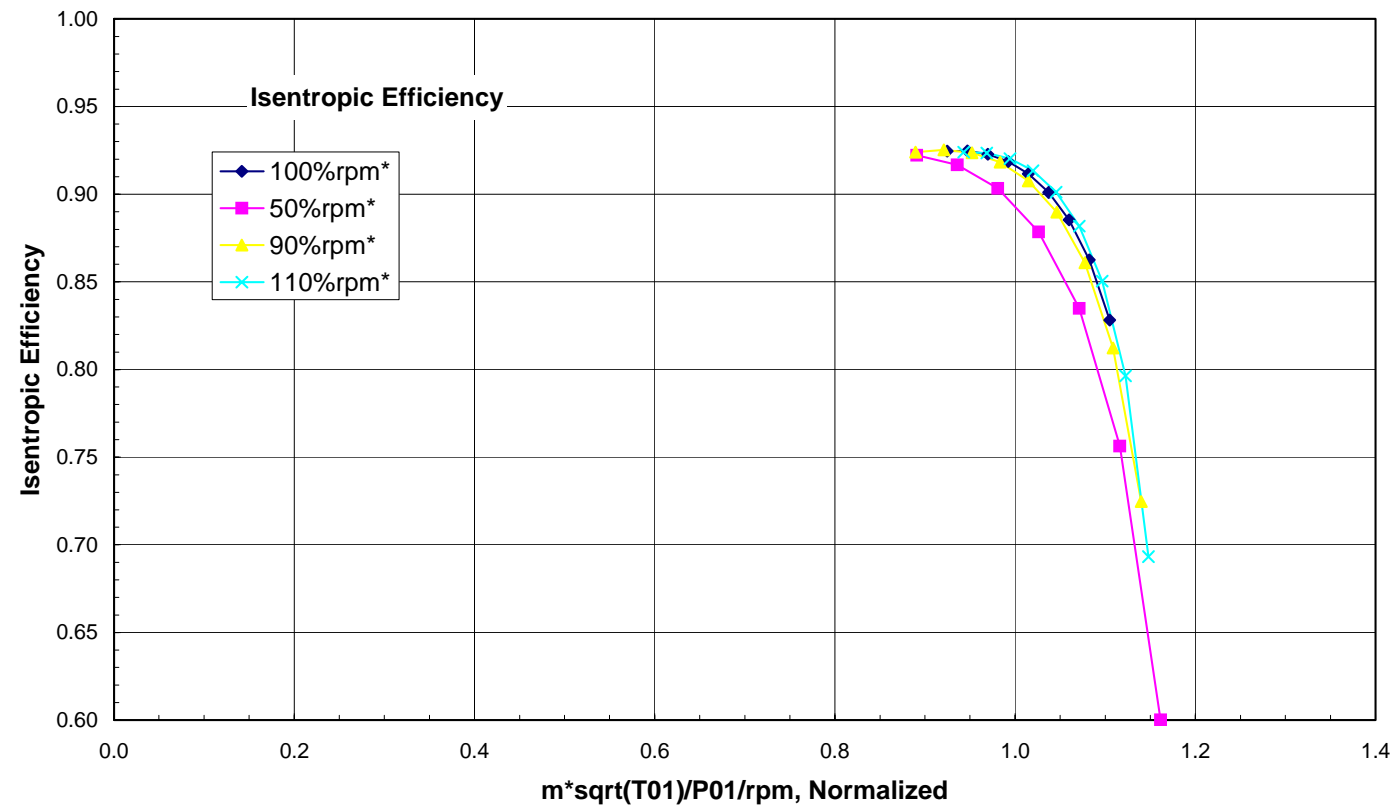

Figure B.7 - Estimated Isentropic Efficiency Versus Corrected Flow Normalized to an 8+1 Axi-Centrifugal Compressor Design Point 


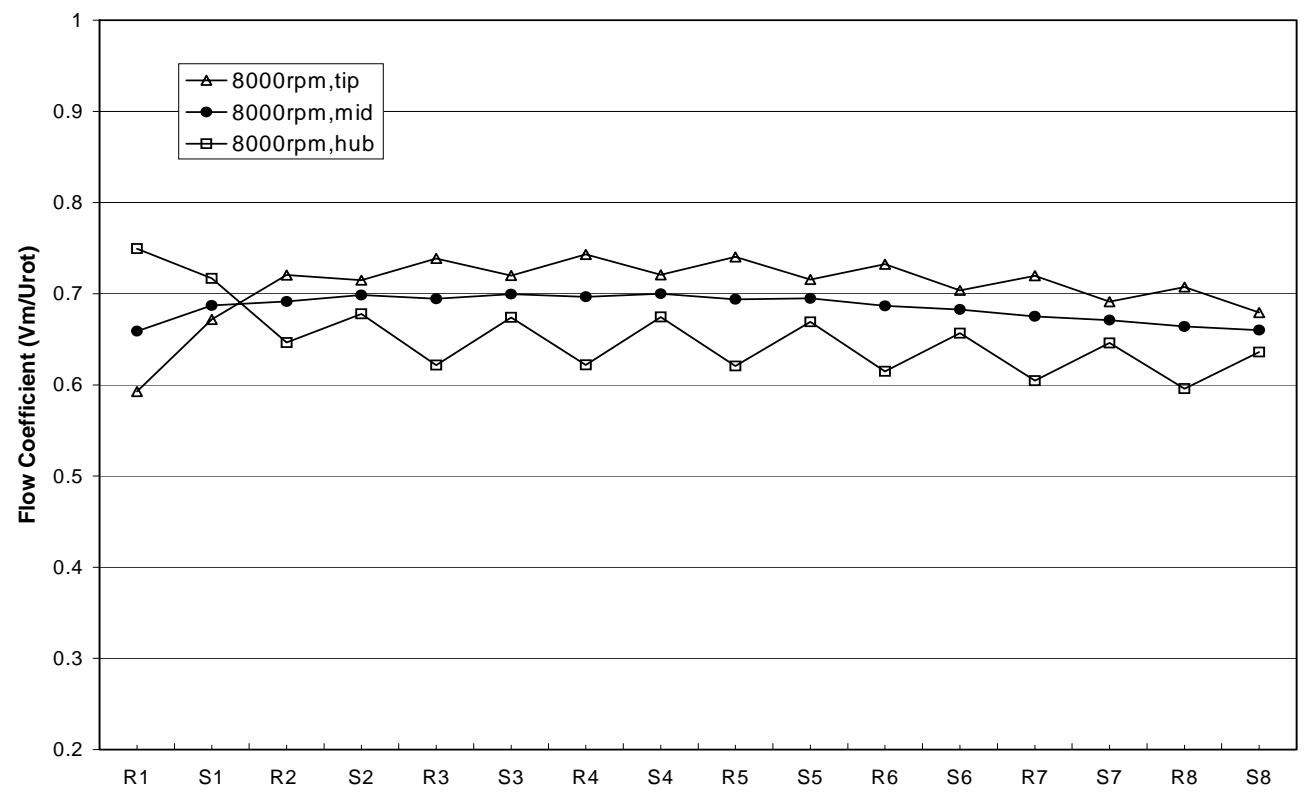

Figure B.8 - Flow Coefficient Per Axial Stage Rotor and Axial Stage Stator at a Tip Speed of $319.2 \mathrm{M} / \mathrm{S}$

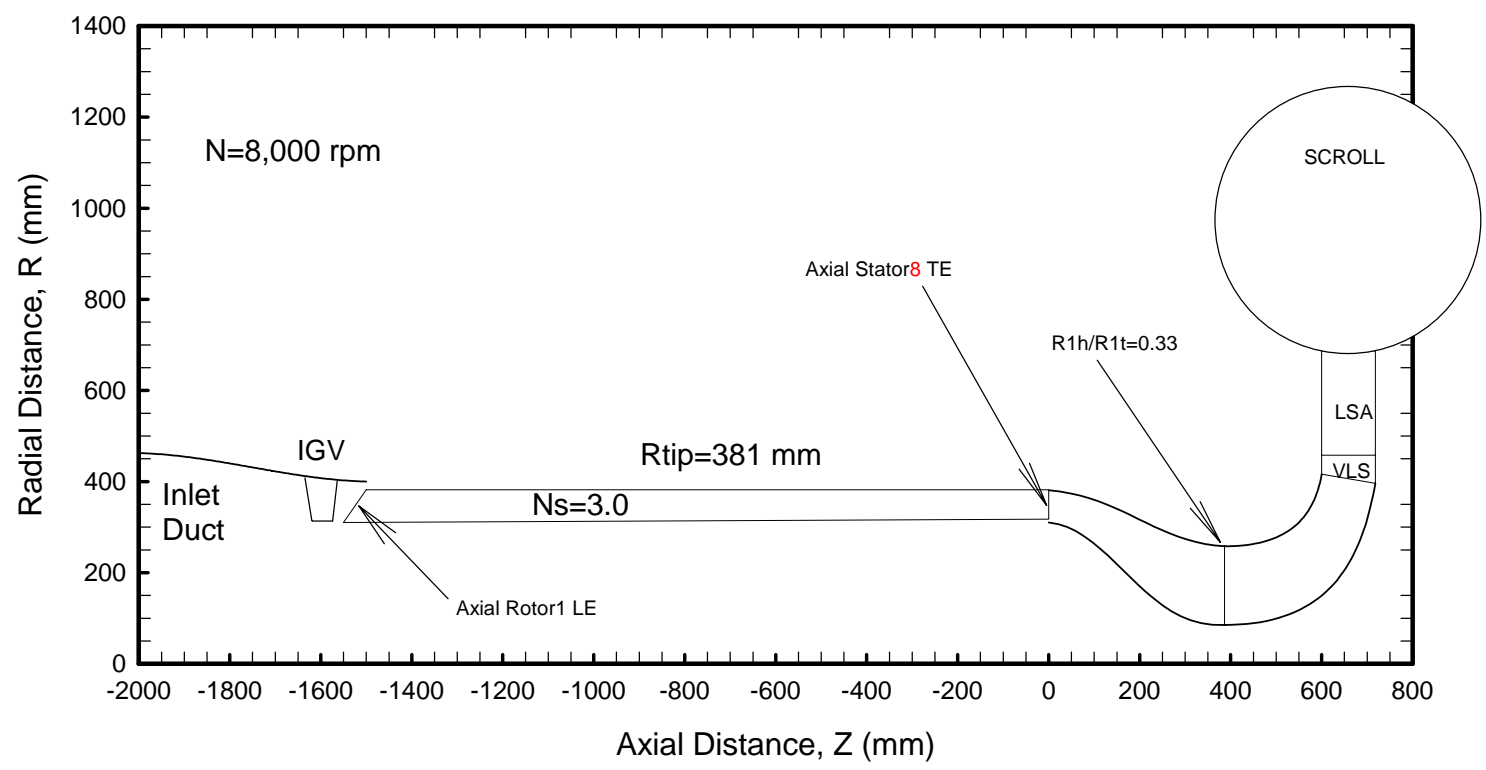

Figure B.9 - Meridional View of an Axi-Centrifugal Flowpath 
Table B.8 - Sizes of HP Turbine and LP Turbine

\begin{tabular}{|c|c|c|c|c|}
\hline $\begin{array}{c}\text { Number of } \\
\text { Turbines }\end{array}$ & $\begin{array}{c}\text { Stages per } \\
\text { Turbine }\end{array}$ & $\begin{array}{c}\text { Optimal } \\
\text { Shaft Speed }\end{array}$ & $\begin{array}{c}\text { Casing Inner } \\
\text { Diameter }\end{array}$ & $\begin{array}{c}\text { Compressor } \\
\text { Casing Length }\end{array}$ \\
\hline 2 & 4 & $8000 \mathrm{rpm}$ & $1.3 \mathrm{~m}$ & $2.0 \mathrm{~m}$ \\
\hline
\end{tabular}

Table B.9 - HP Turbine and LP Turbine Costs

\begin{tabular}{|c|c|c|c|}
\hline & $\begin{array}{c}\text { Recurring } \\
\text { Cost (\$US) }\end{array}$ & $\begin{array}{c}\text { Development/ } \\
\text { Non-recurring } \\
\text { Cost (\$US) }\end{array}$ & Total (\$US) \\
\hline $1^{\text {st }}$ Turbine & 6.0 & 2.6 & 8.6 \\
\hline $2^{\text {nd }}$ Turbine & 5.0 & - & 5.0 \\
\hline
\end{tabular}

The estimated costs in millions of 2001 \$US.

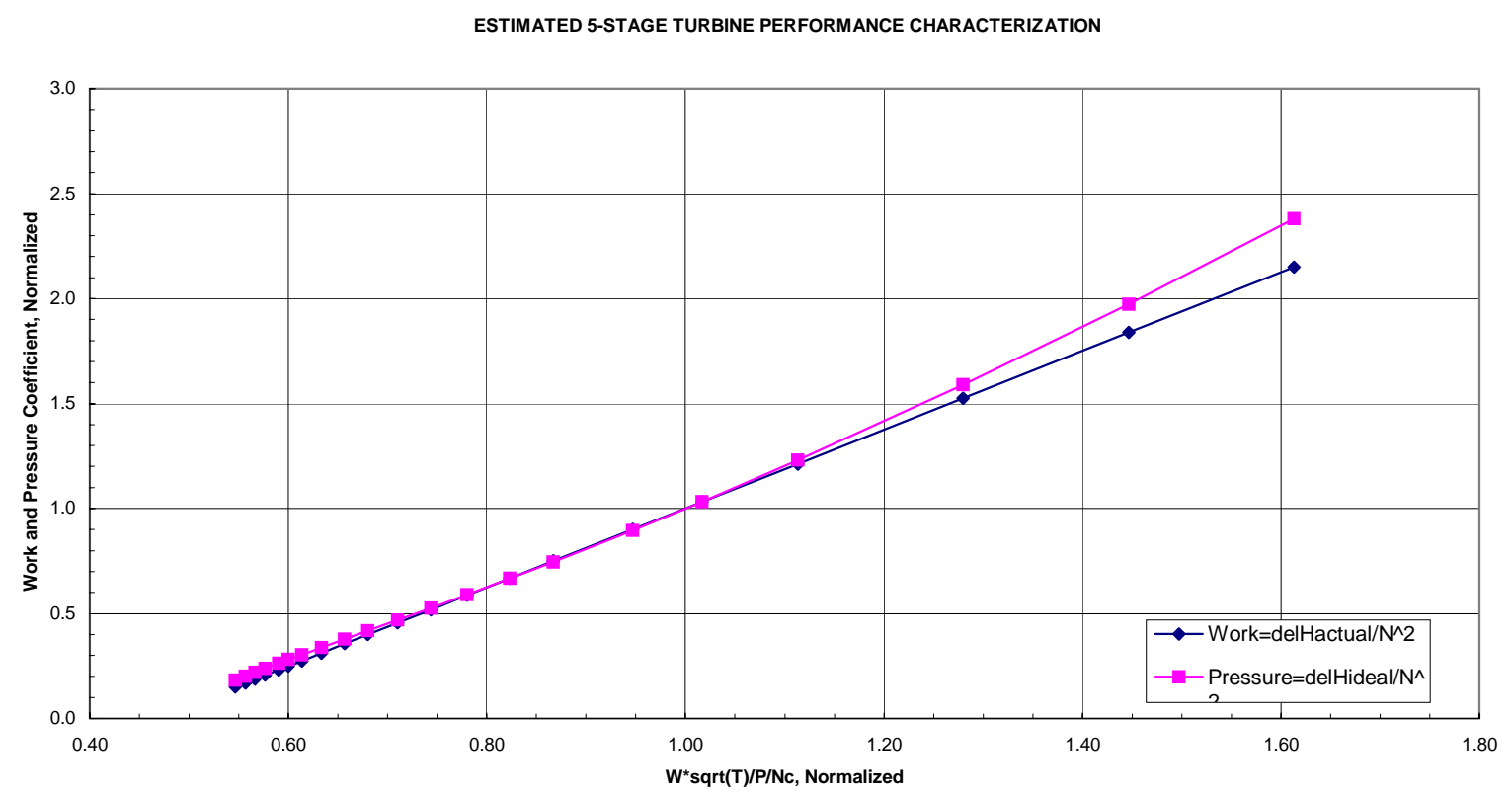

Figure B.10 - Estimated Work and Pressure Coefficients Versus Flow Coefficient Normalized to the Design Point Performance of a Four-Stage Axial Turbine 


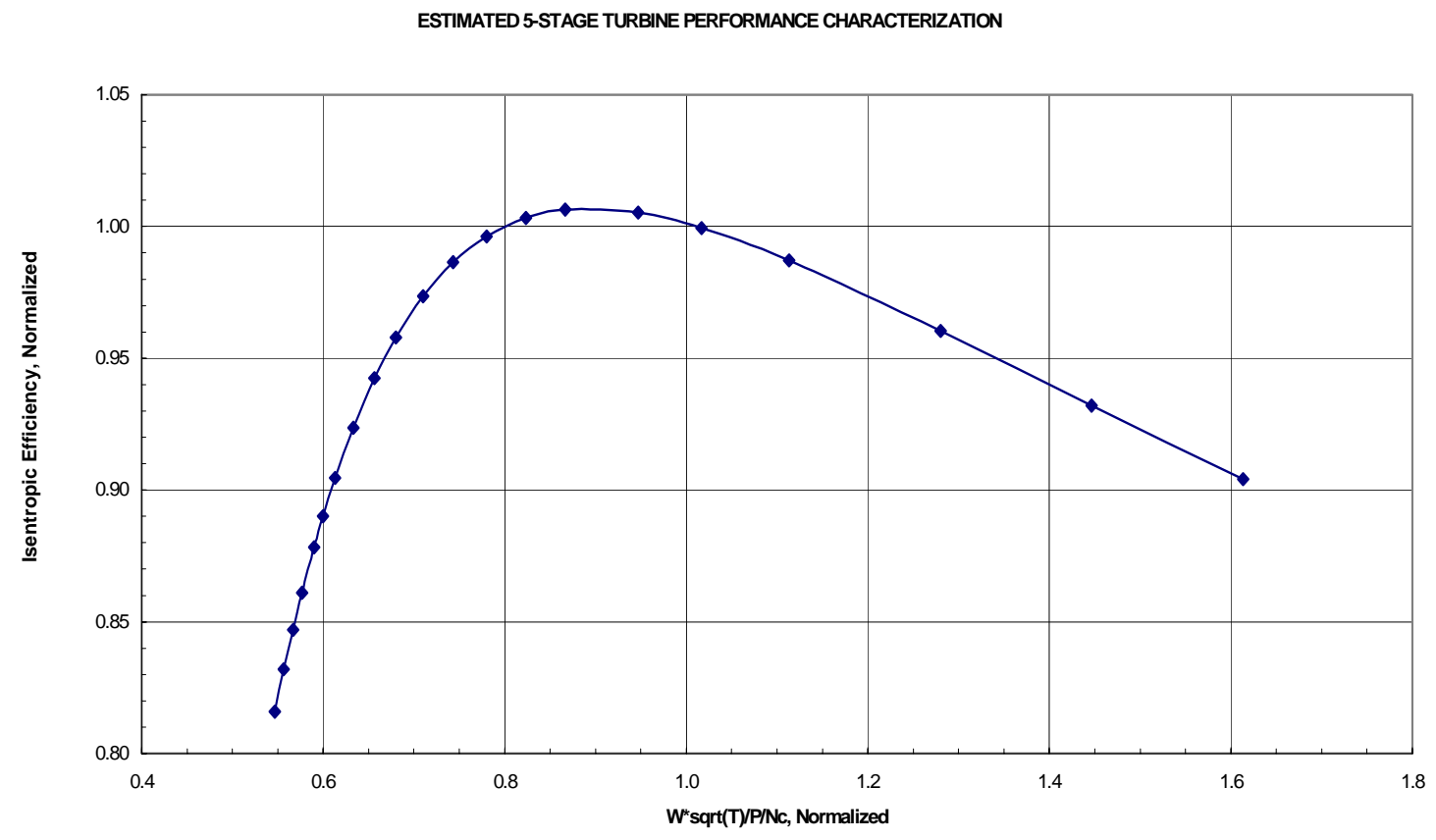

Figure B.11 - Estimated Isentropic Efficiency Versus Flow Coefficient Normalized to the Design Point Performance of a Four-Stage Axial Turbine

Table B.10 -Power Turbine Size

\begin{tabular}{|c|c|c|c|c|}
\hline $\begin{array}{c}\text { Number of } \\
\text { Turbines }\end{array}$ & $\begin{array}{c}\text { Stages per } \\
\text { Turbine }\end{array}$ & $\begin{array}{c}\text { Optimal } \\
\text { Shaft Speed }\end{array}$ & $\begin{array}{c}\text { Casing Inner } \\
\text { Diameter }\end{array}$ & $\begin{array}{c}\text { Compressor } \\
\text { Casing Length }\end{array}$ \\
\hline 1 & 23 & $3600 \mathrm{rpm}$ & $2.9 \mathrm{~m}$ & $6.8 \mathrm{~m}$ \\
\hline
\end{tabular}

Table B.11 - Power Turbine Costs

\begin{tabular}{|c|c|c|c|}
\hline & $\begin{array}{c}\text { Recurring } \\
\text { Cost (\$US) }\end{array}$ & $\begin{array}{c}\text { Development/ } \\
\text { Non-recurring } \\
\text { Cost (\$US) }\end{array}$ & Total (\$US) \\
\hline $1^{\text {st }}$ Turbine & 12 & 3.1 & 15.1 \\
\hline
\end{tabular}

The estimated costs in millions of $2001 \$ U S$. 
Table B.12 Five-Stage Centrifugal Compressor Design Input Data

\title{
Stage 1
}

Input Review

\author{
Compressor Stage Elements \\ SIMPLE IGV \\ IMPELLER \\ VANELESS SPACE \\ DESWIRL SYSTEM
}

\section{Compressor Geometry}

\begin{tabular}{|c|c|c|}
\hline \multicolumn{3}{|l|}{ Igv } \\
\hline Inlet Flange Radius (Hub) & .00000 & $\mathrm{~m}$ \\
\hline Inlet Flange Radius (Shroud) & .31500 & $\mathrm{~m}$ \\
\hline \multicolumn{3}{|l|}{ Impeller } \\
\hline Inlet Radius (Hub) & .14200 & $\mathrm{~m}$ \\
\hline Inlet radius (Shroud) & .37000 & $\mathrm{~m}$ \\
\hline Inlet blade angle (Hub) & -42.00 & \\
\hline Inlet blade angle (Shroud) & -60.00 & $\operatorname{deg}$ \\
\hline Number of blades & 17. & \\
\hline Inlet av normal blade thkns & .00001 & $\mathrm{~m}$ \\
\hline Inlet width & .22880 & $\mathrm{~m}$ \\
\hline LE tip included angle & .00 & $\operatorname{deg}$ \\
\hline Normal geom throat area & .23157 & $\mathrm{~m}^{2}$ \\
\hline Shroud clearance & $=.000800$ & $\mathrm{~m}$ \\
\hline Axial length & .22800 & $\mathrm{~m}$ \\
\hline Tip Radius & .57000 & $\mathrm{~m}$ \\
\hline Tip Width & .12200 & $\mathrm{~m}$ \\
\hline Exit Blade Angle & -40.00 & $\operatorname{deg}$ \\
\hline Exit Av Normal Blade Thkns & .02580 & $\mathrm{~m}$ \\
\hline \multicolumn{3}{|l|}{ Vaneless Diffuser } \\
\hline Radius Ratio & \multirow{2}{*}{\multicolumn{2}{|c|}{ Diffuser Width/Impeller Tip Width }} \\
\hline 1.0000 & & \\
\hline 1.4500 & \multicolumn{2}{|l|}{$\begin{array}{l}1.0000 \\
1.0000\end{array}$} \\
\hline \multicolumn{3}{|l|}{ Deswirl Vanes } \\
\hline \multicolumn{3}{|l|}{ Radius at extra station } \\
\hline Inlet radius/Imp tip radius & $=1.44946$ & \\
\hline
\end{tabular}


Width at extra station $\quad=1.00000$

Inlet width $\quad=1.00000$

Exit radius/Imp tip radius $=.50108$

Exit width $\quad=1.36348$

Turning angle at inlet $\quad=180.000 \mathrm{deg}$

Turning angle at exit $=-90.000 \mathrm{deg}$

Blade angle at inlet set equal to flow angle

Blade angle at exit $=\quad .000 \mathrm{deg}$

Radius ratio of turning (5-7) $=1.500$

Radius ratio of turning (7-8) $=999.000$

Meridional length (5-7) $\quad=.00100 \mathrm{~m}$

Meridional length (7-8) $=.20000 \mathrm{~m}$

Number of vanes (stations 7-8) $=\quad 16.0$

Subtended angle $($ LE to TE) $=25.000 \mathrm{deg}$

Trailing edge (Unblocked

/Total) area ratio, station $8=\quad .00000$

Stage 2

\section{Input Review}

\section{Compressor Stage Elements}

IMPELLER

VANELESS SPACE

DESWIRL SYSTEM

Compressor Geometry

$\begin{array}{llrl}\text { Impeller } & = & .14200 & \mathrm{~m} \\ \text { Inlet Radius (Hub) } & = & .36600 & \mathrm{~m} \\ \text { Inlet radius (Shroud) } & = & -42.00 \\ \text { Inlet blade angle (Hub) } & & -60.00 & \mathrm{deg} \\ \text { Inlet blade angle (Shroud) } & = & 17 . \\ \text { Number of blades } & & .00001 \mathrm{~m} \\ \text { Inlet av normal blade thkns } & = & .22480 & \mathrm{~m} \\ \text { Inlet width } & = & .00 & \mathrm{deg} \\ \text { LE tip included angle } & = & .22574 & \mathrm{~m} \\ \text { Normal geom throat area } & = & .000800 & \mathrm{~m} \\ \text { Shroud clearance } & = & .22800 & \mathrm{~m} \\ \text { Axial length } & = & .57000 & \mathrm{~m} \\ \text { Tip Radius } & = & .11600 & \mathrm{~m} \\ \text { Tip Width } & = & -40.00 & \mathrm{deg} \\ \text { Exit Blade Angle } & & .02580 & \mathrm{~m}\end{array}$

\section{Vaneless Diffuser}

Radius Ratio

Diffuser Width/Impeller Tip Width 


\section{Deswirl Vanes}

Radius at extra station

/imp tip radius $=1.82000$

Inlet radius/Imp tip radius $\quad=1.44946$

Width at extra station $\quad=1.00000$

Inlet width $\quad=1.00000$

Exit radius/Imp tip radius $\quad=\quad .50108$

Exit width $\quad=1.36348$

Turning angle at inlet $\quad=180.000 \mathrm{deg}$

Turning angle at exit $=-90.000 \mathrm{deg}$

Blade angle at inlet set equal to flow angle

Blade angle at exit $=\quad .000 \mathrm{deg}$

Radius ratio of turning (5-7) $=1.500$

Radius ratio of turning (7-8) $=999.000$

Meridional length (5-7) $=.00100 \mathrm{~m}$

Meridional length (7-8) $=.20000 \mathrm{~m}$

$\begin{array}{lrr}\text { Number of vanes (stations 7-8) } & =16.0 \\ \text { Subtended angle (LE to TE) } & =25.000 \mathrm{deg}\end{array}$

Trailing edge (Unblocked

/Total) area ratio, station $8=\quad .00000$

\section{Stage 3}

\section{Input Review}

\section{Compressor Stage Elements}

IMPELLER

VANELESS SPACE

DESWIRL SYSTEM

\section{Compressor Geometry}

$\begin{array}{llrl}\text { Impeller } & & & \\ \text { Inlet Radius (Hub) } & = & .36000 & \mathrm{~m} \\ \text { Inlet radius (Shroud) } & = & -42.00 & \\ \text { Inlet blade angle (Hub) } & = & -60.00 & \mathrm{deg} \\ \text { Inlet blade angle (Shroud) } & = & 17 . & \\ \text { Number of blades } & = & .00001 & \mathrm{~m} \\ \text { Inlet av normal blade thkns } & & .21880 & \mathrm{~m}\end{array}$




$\begin{array}{llrl}\text { LE tip included angle } & = & .00 & \mathrm{deg} \\ \text { Normal geom throat area } & = & .21712 & \mathrm{~m}^{2} \\ \text { Shroud clearance } & = & .000800 & \mathrm{~m} \\ \text { Axial length } & = & .22800 & \mathrm{~m} \\ \text { Tip Radius } & = & .57000 & \mathrm{~m} \\ \text { Tip Width } & = & .11100 & \mathrm{~m} \\ \text { Exit Blade Angle } & = & -40.00 & \mathrm{deg} \\ \text { Exit Av Normal Blade Thkns } & = & .02580 & \mathrm{~m} \\ & & & \\ \text { Vaneless Diffuser } & & & \\ \text { Radius Ratio } & \text { Diffuser Width/Impeller Tip Width } \\ \quad 1.0000 & & 1.0000 \\ 1.4500 & & 1.0000\end{array}$

\section{Deswirl Vanes}

Radius at extra station /imp tip radius $=1.82000$

Inlet radius/Imp tip radius $=1.44946$

Width at extra station $\quad=1.00000$

Inlet width $\quad=1.00000$

Exit radius $/$ Imp tip radius $\quad=\quad .50108$

Exit width $=1.36348$

Turning angle at inlet $\quad=180.000 \mathrm{deg}$

Turning angle at exit $=-90.000 \mathrm{deg}$

Blade angle at inlet set equal to flow angle

Blade angle at exit $=\quad .000 \mathrm{deg}$

Radius ratio of turning $(5-7)=1.500$

Radius ratio of turning (7-8) $=999.000$

Meridional length (5-7) $=.00100 \mathrm{~m}$

Meridional length (7-8) $=.20000 \mathrm{~m}$

Number of vanes (stations 7-8) $=\quad 16.0$

Subtended angle (LE to TE) $=25.000 \mathrm{deg}$

Trailing edge (Unblocked

/Total) area ratio, station $8=\quad .00000$

Stage 4

Input Review

Compressor Stage Elements

IMPELLER

VANELESS SPACE

DESWIRL SYSTEM 


\section{Compressor Geometry}

\begin{tabular}{|c|c|c|}
\hline \multicolumn{3}{|l|}{ Impeller } \\
\hline Inlet Radius (Hub) & .14200 & $\mathrm{~m}$ \\
\hline Inlet radius (Shroud) & .35600 & $\mathrm{~m}$ \\
\hline Inlet blade angle (Hub) & -42.00 & \\
\hline Inlet blade angle (Shroud) & -60.00 & deg \\
\hline Number of blades & 17. & \\
\hline Inlet av normal blade thkns & .00001 & $\mathrm{~m}$ \\
\hline Inlet width & .21480 & $\mathrm{~m}$ \\
\hline LE tip included angle & .00 & deg \\
\hline Normal geom throat area & .21145 & $\mathrm{~m}^{2}$ \\
\hline Shroud clearance & $=.000800$ & $\mathrm{~m}$ \\
\hline Axial length & .22800 & $\mathrm{~m}$ \\
\hline Tip Radius & .56000 & $\mathrm{~m}$ \\
\hline Tip Width & .10800 & $\mathrm{~m}$ \\
\hline Exit Blade Angle & -40.00 & $\operatorname{deg}$ \\
\hline Exit Av Normal Blade Thkns & .02580 & $\mathrm{~m}$ \\
\hline Vaneless Diffuser & & \\
\hline Radius Ratio & Diffuser Wid & th/Impeller Tip Width \\
\hline 1.0000 & 1.0000 & \\
\hline 1.4500 & 1.0000 & \\
\hline
\end{tabular}

\section{Deswirl Vanes}

Radius at extra station

$$
\text { /imp tip radius }=1.82000
$$

Inlet radius/Imp tip radius $\quad=1.44946$

Width at extra station $\quad=1.00000$

Inlet width $\quad=1.00000$

Exit radius/Imp tip radius $=.50108$

Exit width $\quad=1.36348$

Turning angle at inlet $\quad=180.000 \mathrm{deg}$

Turning angle at exit $=-90.000 \mathrm{deg}$

Blade angle at inlet set equal to flow angle

Blade angle at exit $=\quad .000 \mathrm{deg}$

Radius ratio of turning (5-7) $=1.500$

Radius ratio of turning (7-8) $=999.000$

Meridional length (5-7) $\quad=.00100 \mathrm{~m}$

Meridional length (7-8) $\quad=.20000 \mathrm{~m}$

Number of vanes (stations 7-8) $=\quad 16.0$

Subtended angle $(\mathrm{LE}$ to TE) $=25.000 \mathrm{deg}$

Trailing edge (Unblocked

/Total) area ratio, station $8=\quad .00000$ 


\section{Stage 5}

\section{Input Review}

\section{Compressor Stage Elements \\ IMPELLER \\ VANELESS SPACE \\ SPECIFIED LOSS-CONST}

\section{Compressor Geometry}

\begin{tabular}{|c|c|c|}
\hline \multicolumn{3}{|l|}{ Impeller } \\
\hline Inlet Radius (Hub) & .14200 & $\mathrm{~m}$ \\
\hline Inlet radius (Shroud) & .35000 & $\mathrm{~m}$ \\
\hline Inlet blade angle (Hub) & -42.00 & \\
\hline Inlet blade angle (Shroud) & -60.00 & $\operatorname{deg}$ \\
\hline Number of blades & 17. & \\
\hline Inlet av normal blade thkns & .00001 & $\mathrm{~m}$ \\
\hline Inlet width & .20880 & $\mathrm{~m}$ \\
\hline LE tip included angle & .00 & $\operatorname{deg}$ \\
\hline Normal geom throat area & .20307 & $\mathrm{~m}^{2}$ \\
\hline Shroud clearance & .000800 & $\mathrm{~m}$ \\
\hline Axial length & .22800 & $\mathrm{~m}$ \\
\hline Tip Radius & .57000 & $\mathrm{~m}$ \\
\hline Tip Width & .10100 & $\mathrm{~m}$ \\
\hline Exit Blade Angle & -40.00 & $\operatorname{deg}$ \\
\hline Exit Av Normal Blade Thkns & .02580 & $\mathrm{~m}$ \\
\hline \multicolumn{3}{|l|}{ Vaneless Diffuser } \\
\hline Radius Ratio & \multicolumn{2}{|c|}{ Diffuser Width/Impeller Tip Width } \\
\hline 1.0000 & \multicolumn{2}{|c|}{1.0000} \\
\hline 1.4500 & \multicolumn{2}{|l|}{1.0000} \\
\hline
\end{tabular}

\section{Exit}

Discharge Pipe Entry Area $=.24200 \mathrm{~m}^{2}$ 


\section{Appendix C Intermediate Heat Exchanger Assembly Design}

The IHX design using a plate-fin configuration consists of 18 identical modules. The layout of the plate-fin IHX was designed by Mr. Pete Stahle of MIT. 6 Vessels are arranged to envelop the 18 modules. Each vessel holds three IHX modules as shown in Figures C.1 to C.4. The vessel is $90.5 \mathrm{in}$. in diameter, $2 \mathrm{in}$. in thickness, $240 \mathrm{in}$. in height and 90,000 lb in weight. The

arrangement of three modules in one vessel is shown in Figure C.2. Figure C.3 shows the primary side internals; the modules are suspended to accommodate thermal expansion. Figure C.4 illustrates the secondary side internals. Since the secondary side is an ASME code III pressure boundary, the temperature limitation is $427^{\circ} \mathrm{C}$. Thus insulation and a cooling stream must be provided to ensure the pressure boundary temperature is lower than the limitation. The IHX assembly is shown in Figures C.5 and C.6. Figure C.7 demonstrates the assembly piping.

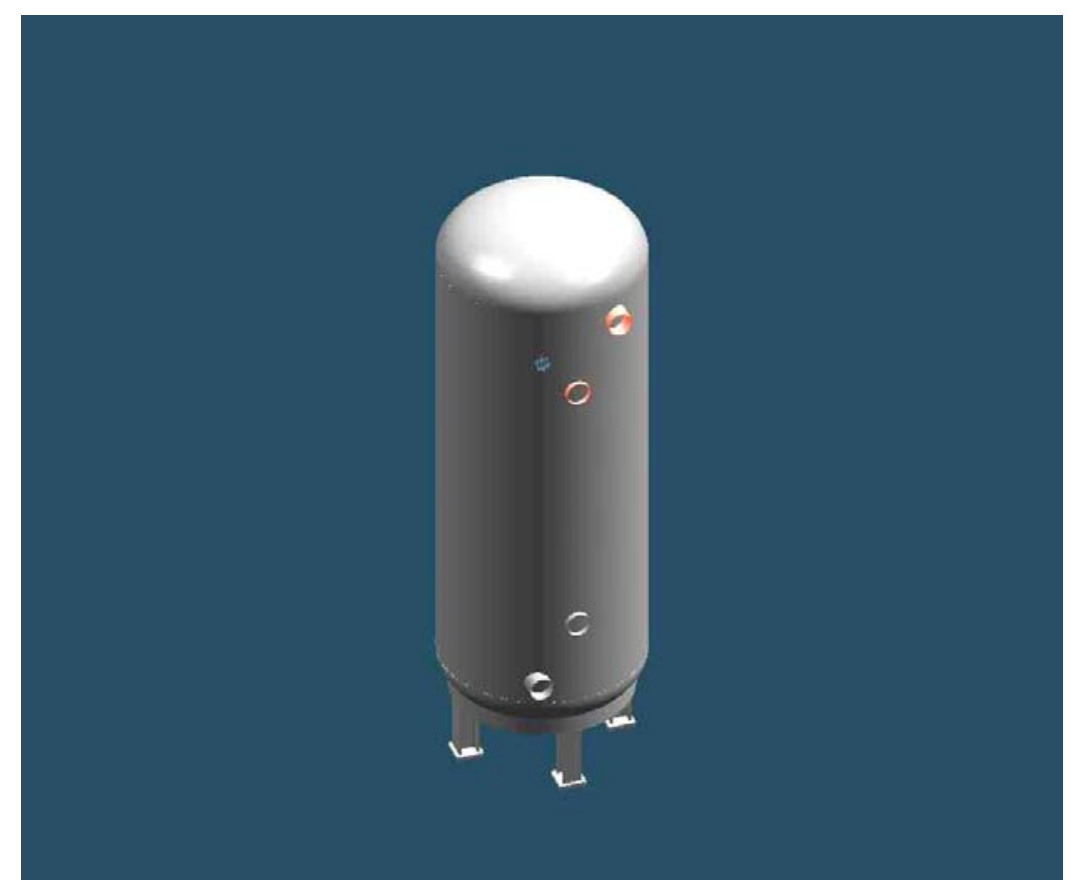

Figure C.1 - IHX unit pressure vessel 


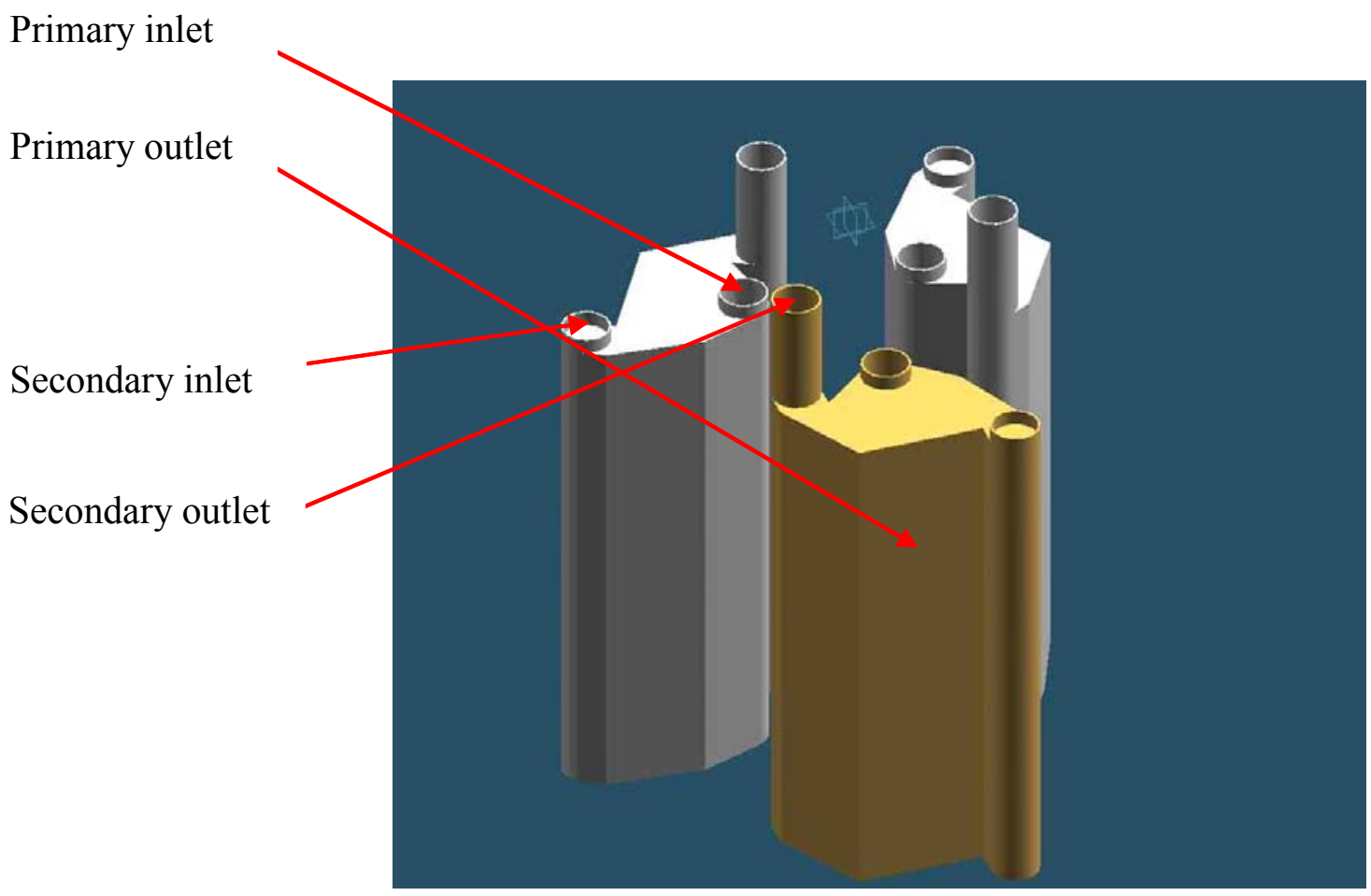

Figure - C.2 Plate-fin grouping in one vessel

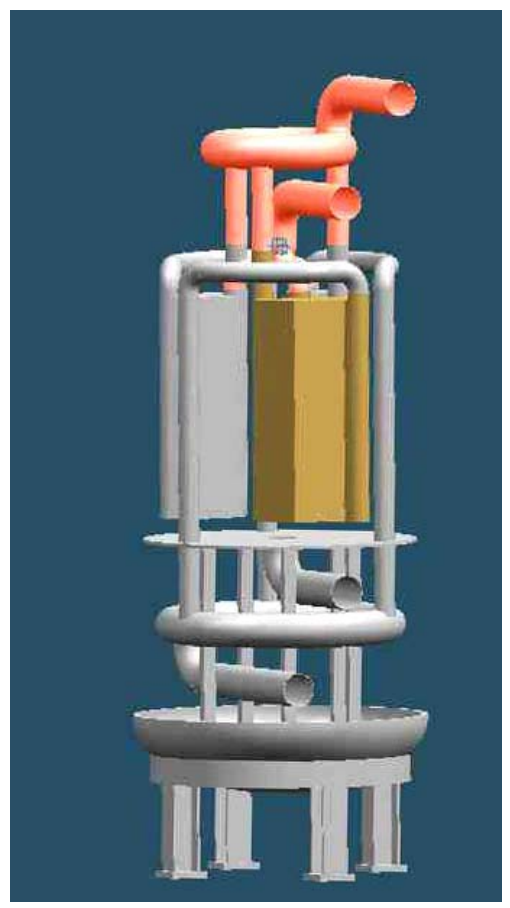

Figure C.3 - Primary side internals 


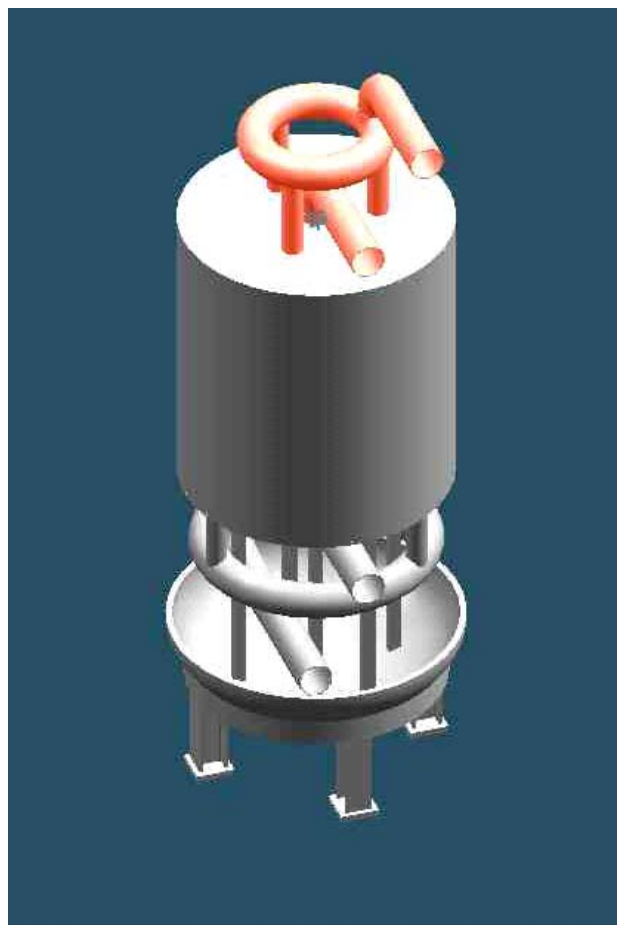

Figure C.4 - Secondary side internals

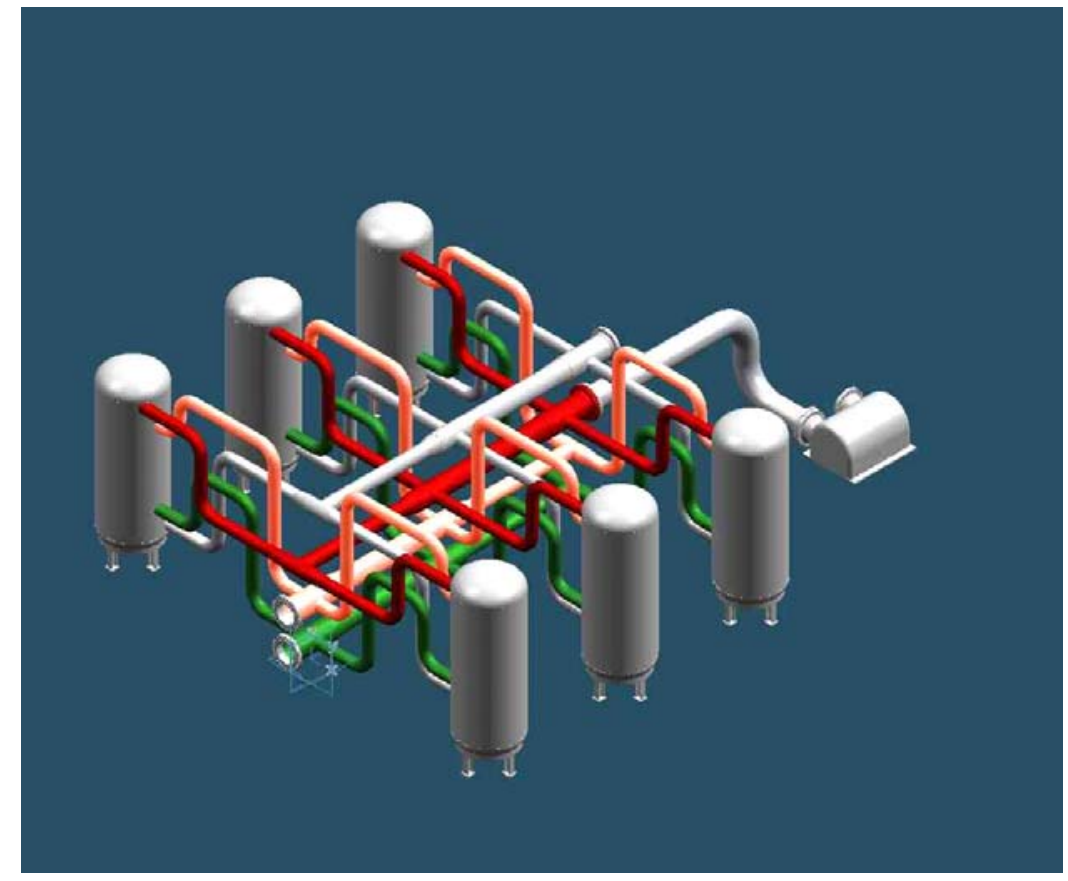

Figure C.5 - IHX assembly isometric view 


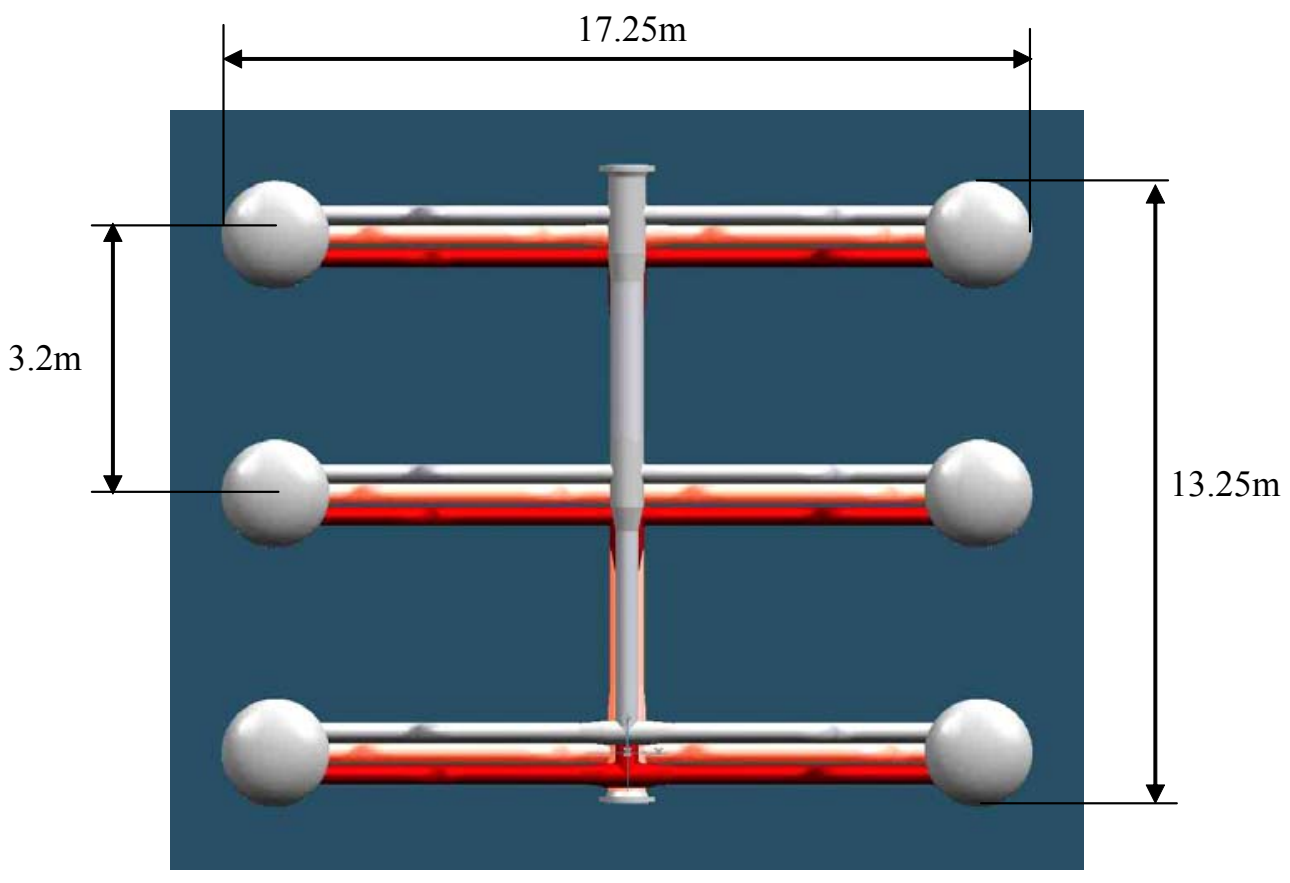

Figure C.6 - IHX assembly plan view

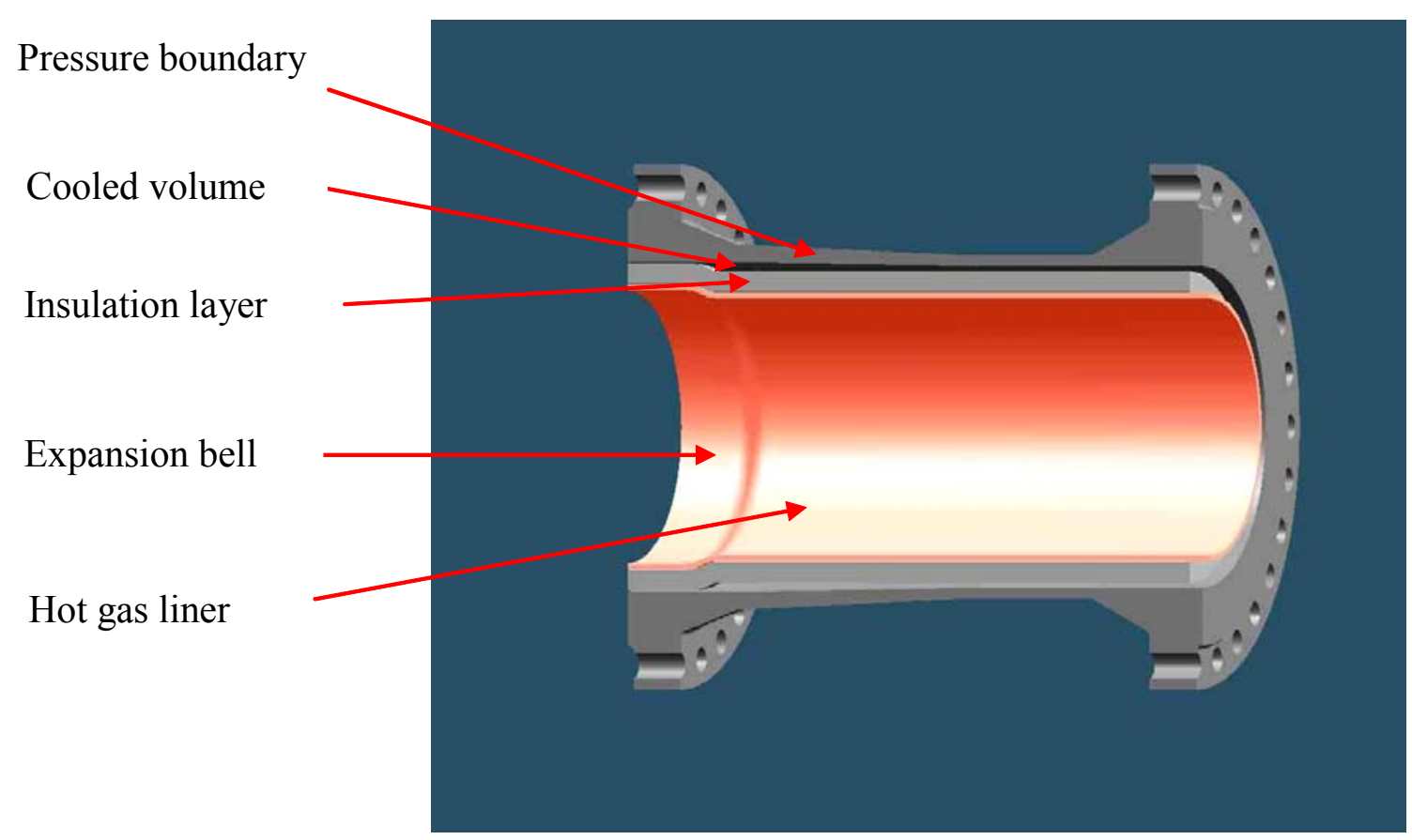

Figure C.7 - IHX assembly piping 


\section{Appendix D Thermodynamic and transport properties of helium}

\section{Thermodynamic and Transport Properties of Helium}

$$
\left(273-1500{ }^{\circ} \mathrm{K}, 0.1-10 \mathrm{MPa}\right)
$$

1. Gas constant

$$
\mathrm{R}=2077.22\left(\mathrm{~J} / \mathrm{kg}{ }^{\circ} \mathrm{K}\right)
$$

Uncertainty

$<0.05 \%$

2. Equation of State

$$
\begin{aligned}
& \mathrm{PV}=\mathrm{RT}+\mathrm{PB}(\mathrm{T}) \quad \text { Where } \\
& B(T)=C_{1}+\frac{C_{2}}{1-C_{3} T}+\frac{C_{4}}{1+C_{5} T} \\
& C_{1}=9.489433 * 10^{-4}\left(\mathrm{~m}^{3} / \mathrm{kg}\right) \\
& C_{2}=9.528079 * 10^{-4}\left(\mathrm{~m}^{3} / \mathrm{kg}\right) \\
& C_{3}=3.420680 * 10^{-2}\left(\mathrm{~K}^{-1}\right) \\
& C_{4}=2.739470 * 10^{-3}\left(\mathrm{~m}^{3} / \mathrm{kg}\right) \\
& C_{5}=9.409120 * 10^{-4}\left(\mathrm{~K}^{-1}\right)
\end{aligned}
$$

3. Compressibility $Z=1+\frac{P}{R T} B(T)$

4. Specific Heat
$\mathrm{C}_{\mathrm{P}}=5193.0(\mathrm{~J} / \mathrm{kg} \mathrm{K})$
$<0.5 \%$
$\mathrm{C}_{\mathrm{v}}=3116.0(\mathrm{~J} / \mathrm{kg} \mathrm{K})$
$<0.5 \%$

5. Enthalpy $H-H_{0}=C_{P} T+\left[B(T)-T \frac{d}{d T} B(T)\right] P$ $<1.0 \%$

6. Entropy

$S-S_{0}=C_{P} \ln \left(T / T_{0}\right)-R \ln \left(P / P_{0}\right)-P \frac{d}{d T} B(T)$

$<1.0 \%$

7. Sonic Velocity

$c=Z \sqrt{\gamma R T}$

$<1.0 \%$

8. Viscosity

$\eta=3.953 * 10^{-7} T^{0.667}\left(N-s / m^{2}\right)$

$\sigma_{\eta=1.5 \%}$

9. Thermal Conductivity

$\mathrm{k}=2.774 * 10^{-3} \mathrm{~T}^{0.701}(\mathrm{~W} / \mathrm{m} \mathrm{K})$

$\sigma_{\mathrm{k}=2.4 \%}$

10. Prandtl Number

$$
\operatorname{Pr}=\frac{\eta C_{P}}{k}=0.740 T^{-0.014} \text { where } \mathrm{T} \text { in }(\mathrm{K})
$$

$<3.0 \%$ 


\section{Appendix F Nomenclature}

Most of the nomenclature is defined when it is introduced or else is obvious from the context of to use. Here it is summarized for convenience.

Roman Letter Symbols

\begin{tabular}{|l|l|}
\hline$A$ & Cross section area \\
\hline $\mathrm{A}_{\mathrm{c}}$ & Empty core cross-sectional area \\
\hline $\mathrm{C}$ & Velocity \\
\hline $\mathrm{C}_{\mathrm{i}}$ & Concentration of delayed precursors of group I \\
\hline $\mathrm{Cp}$ & Specific heat at constant pressure \\
\hline $\bar{C} p$ & Average specific heat at constant pressure \\
\hline $\mathrm{C} \mathrm{V}$ & Specific heat at constant volume \\
\hline $\mathrm{D}$ & Diameter \\
\hline $\mathrm{D}_{\mathrm{e}}$ & Hydraulic diameter \\
\hline $\mathrm{D}_{\mathrm{H}}$ & Heated diameter \\
\hline $\mathrm{d}_{\mathrm{p}}$ & Pebble bed diameter \\
\hline $\mathrm{e}(\mathrm{t})$ & Error signal of PI controller \\
\hline $\mathrm{f}$ & Friction factor \\
\hline $\mathrm{h}$ & Enthalpy \\
\hline $\mathrm{h}$ & Heat transfer coefficient \\
\hline $\mathrm{H}$ & Height \\
\hline $\mathrm{I}$ & Concentration of Iodine \\
\hline $\mathrm{I}$ & Inertia \\
\hline $\mathrm{K}$ & Coefficient \\
\hline $\mathrm{K}_{\mathrm{P}}$ & Proportional gain of PI controller \\
\hline $\mathrm{k}$ & Conductivity \\
\hline $\mathrm{k}_{\mathrm{eff}}$ & Effective multiplication factor \\
\hline $\mathrm{L}$ & Nodal axial height \\
\hline $\mathrm{L}$ & Pipe length \\
\hline $\mathrm{L}_{\theta}$ & Heat transfer circumferential length \\
\hline$\dot{m}$ & Mass flowrate \\
\hline $\mathrm{N}$ & Turbomachinery rotational speed \\
\hline $\mathrm{n}$ & Heat exchanger time constant \\
\hline $\mathrm{P}$ & Power \\
\hline $\mathrm{PD}$ & Percentage pressure loss \\
\hline $\mathrm{PR}$ & Pressure ratio \\
\hline $\mathrm{PR}$ & Compressor pressure ratio \\
\hline $\mathrm{p}$ & Pressure \\
\hline $\mathrm{Q}$ & Heat \\
\hline $\mathrm{q}$ & Linear power \\
\hline $\mathrm{R}$ & Radius \\
\hline $\mathrm{R}$ & Gas constant \\
\hline & \\
\hline
\end{tabular}




\begin{tabular}{|l|l|}
\hline$S$ & Neutron source \\
\hline$T$ & Temperature \\
\hline$T_{i}$ & Tuning parameter of PI controller \\
\hline$t$ & Time \\
\hline$U$ & Overall conductance of the heat transfer \\
\hline$U$ & Turbomachinery blade tip speed \\
\hline$u(t)$ & Output singnal of a PI controller \\
\hline$W_{c}$ & Compressor consumed power \\
\hline$W_{t}$ & Turbine generated power \\
\hline$X$ & Concentration of Xe \\
\hline
\end{tabular}

Greek Letter Symbols

\begin{tabular}{|l|l|}
\hline$\alpha_{\mathrm{T}}$ & Rate of reactivity change per unit temperature change \\
\hline$\beta$ & Total effective delayed neutron fraction \\
\hline$\beta_{\mathrm{i}}$ & Effective delayed neutron fraction for group i \\
\hline$\Delta$ & Denotes difference \\
\hline$\Lambda$ & Prompt neutron life time \\
\hline$\Sigma_{\mathrm{f}}$ & Macroscopic fission cross section \\
\hline$\Sigma_{\mathrm{a}}$ & Macroscopic absorption cross section \\
\hline$\Sigma_{a}^{X}$ & Macroscopic absorption cross section of Xe \\
\hline$\delta$ & Cavity width \\
\hline$\varepsilon$ & Emmisivity \\
\hline$\varepsilon$ & Pebble bed void fraction (eqn. 4.16$)$ \\
\hline$\varepsilon_{\mathrm{hx}}$ & Heat exchanger effectiveness \\
\hline$\gamma$ & Cp/Cv \\
\hline$\gamma_{\mathrm{I}}$ & Effective fraction of Iodine \\
\hline$\gamma_{\mathrm{X}}$ & Effective fraction of Xe \\
\hline$\lambda$ & Average decay constant \\
\hline$\lambda_{\mathrm{i}}$ & Decay constant of delayed precursors of group I \\
\hline$\eta_{\mathrm{cycle}}$ & Cycle efficiency \\
\hline$\eta_{\mathrm{m}}$ & Mechanical efficiency \\
\hline$\eta_{\mathrm{net}}$ & Plant net efficiency \\
\hline$\eta_{\mathrm{pc}}$ & Compressor polytropic efficiency \\
\hline$\eta_{\mathrm{pt}}$ & Turbine polytropic efficiency \\
\hline$\eta_{\mathrm{sc}}$ & Compressor isentropic efficiency \\
\hline$\eta_{\mathrm{st}}$ & Turbine isentropic efficiency \\
\hline$\phi$ & Neutron flux density \\
\hline$\sigma$ & Stefan-Boltzman constant \\
\hline$\sigma_{\mathrm{f}}$ & Microscopic fission cross section \\
\hline$\sigma_{a}^{X}$ & Microscopic absorption cross section of Xe \\
\hline$\mu$ & Dynamic viscosity \\
\hline
\end{tabular}




\begin{tabular}{|l|l|}
\hline$\rho$ & Density \\
\hline$\rho$ & Reactivity \\
\hline$\tau$ & Heat exchanger time constant \\
\hline$\omega_{\mathrm{f}}$ & Usable energy released per fission event \\
\hline$v$ & Neutron speed \\
\hline
\end{tabular}

Subscripts
\begin{tabular}{|l|l|}
\hline 0 & Stagnation \\
\hline 1 & Inlet \\
\hline 2 & Outlet \\
\hline c & Cold side \\
\hline cor & Corrected \\
\hline h & Hot side \\
\hline min & Minimum \\
\hline s & Isentropic \\
\hline p & Polytropic \\
\hline
\end{tabular}

Dimensionless Groupings

\begin{tabular}{|l|l|}
\hline $\mathrm{Re}$ & Reynolds number \\
\hline $\mathrm{Pr}$ & Prandtl number \\
\hline $\mathrm{Nu}$ & Nusselt number \\
\hline
\end{tabular}

